\title{
Optimal Vehicle Motion Control to Mitigate Secondary Crashes after an Initial Impact
}

\author{
by \\ Byung-Joo Kim \\ A dissertation submitted in partial fulfillment \\ of the requirements for the degree of \\ Doctor of Philosophy \\ (Mechanical Engineering) \\ in the University of Michigan \\ 2015
}

Doctoral Committee:

Professor Huei Peng, Chair

Professor Ilya V. Kolmanovsky

Professor Noel C. Perkins

Professor Dawn M. Tilbury 
(C) Byung-Joo Kim

2015 


\section{ACKNOWLEDGEMENTS}

I would first like to thank Professor Huei Peng for his support and guidance. His engineering passion inspired me to test myself beyond my limits and allowed me to enjoy the challenges as I developed my expertise in the engineering field. He continually taught me how to be creative, how to approach challenging problems, and how to demonstrate my work to others. Because he guided me in a fascinating direction where I could have engineering passion to make a difference in the world, I was able to continue to be passionate about my research. I would also like to thank the professors on my committee, Professor Noel Perkins, Professor Dawn Tilbury, and Professor Ilya Kolmanovsky. Their advice and comments were greatly helpful to complete this dissertation. I am grateful to them for their time and willingness to provide keen insights that made my work more meaningful. I gratefully acknowledge the support from MANDO Corporation, allowing me to study in the $\mathrm{PhD}$ program, conduct research, and obtain $\mathrm{PhD}$ degree at the University of Michigan.

In addition, I would like to acknowledge many people who supported me to complete this thesis. I am grateful to Dr. Jing Zhou at GM who provided valuable comments and the foundation for my research. Comments and helpful advice from Prof. Timothy Gordon at the University of Lincoln and Dr. Derong Yang at Volvo were invaluable. I feel lucky that I have been a member of Vehicle Dynamics Lab (VDL) and spend time with incredibly smart and warmhearted friends. Many thanks to Dr. Changsun Ahn, Dr. Chiao-Ting Li, Dr. Jongwha Yoon, Dr. William Smith, Tianyou Guo, Xiaowu Zhang, Ding Zhao, Yuxiao Chen, and Su-Yang Shieh. Thoughtful discussion, general help, and friendship were all greatly appreciated. Many thanks to unforgettable years with Jessica Vinter who proofread my writings for papers and thesis. She also helped me learn and enjoy American culture. 
I would like to thank all my family for their unconditional support and love. I am especially grateful to my wife, Kyungsook Hwang. Without her sacrifice and patience, this dissertation and my doctoral study would not have been possible. I always thank her for encouraging me to be a confident person under any circumstances. My heart was always full when I was with my children: Kaeun, Seunghui, and Donghui. Finally, I want to leave a note for my children; when you grow up and become big enough to read this, I hope this achievement could encourage you to keep challenging yourself for your interest and happiness. This is one of my desired results that I am pleased to show you as an example. 


\section{TABLE OF CONTENTS}

ACKNOWLEDGEMENTS ............................................................................................... ii

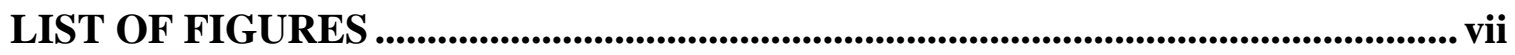

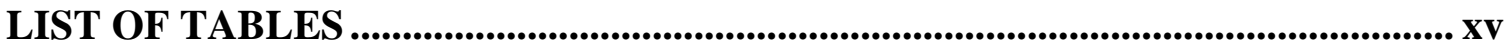

LIST OF SYMBOLS ............................................................................................. $\mathrm{xvi}$

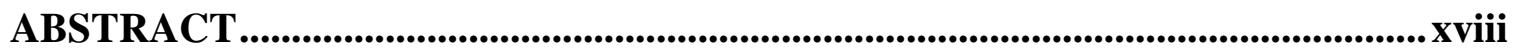

CHAPTER 1 Introduction ................................................................................................ 1

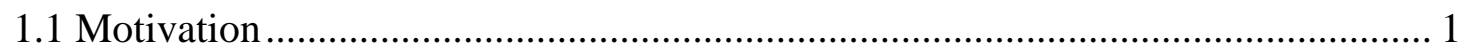

1.1.1 Risk of Lateral Deviation ................................................................... 2

1.1.2 Risk of Vehicle Rotation and Side Impact Crashes .................................. 4

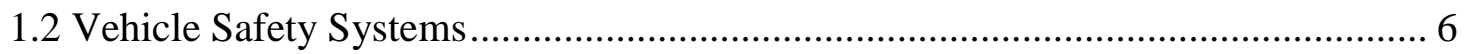

1.3 Controls to Avoid Secondary Collisions .......................................................... 9

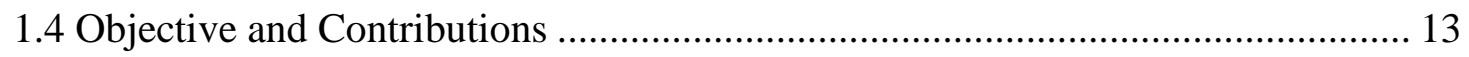

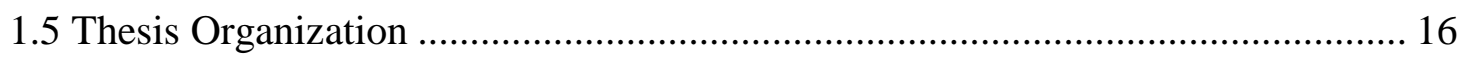

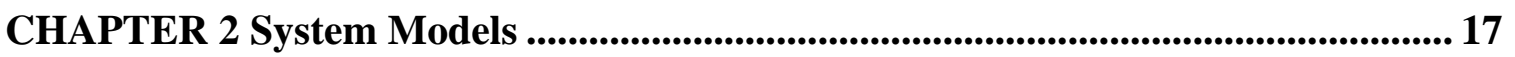

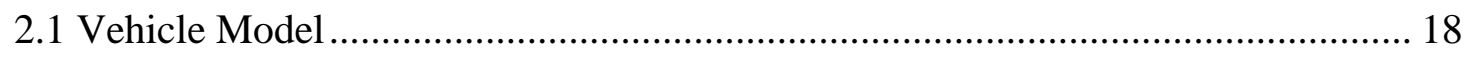

2.1.1 Four Degrees of Freedom Model ............................................................ 19

2.1.2 Three Degrees of Freedom Model ......................................................... 20

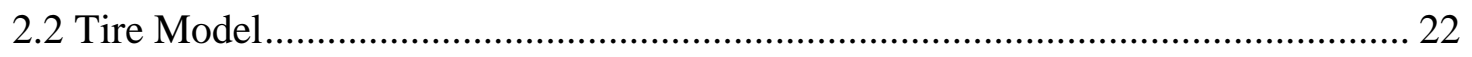

2.2.1 Pacejka Tire Model ................................................................................ 23

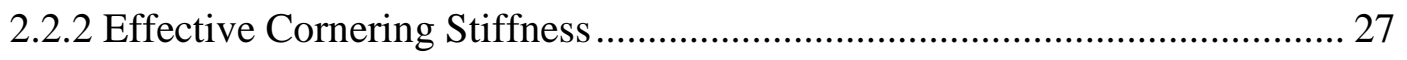


2.3 Parameters for Simulations

CHAPTER 3 Collision Strength Estimation and Vehicle Motion Prediction ........... 33

3.1 Collision Force Profile and Time Duration...................................................... 34

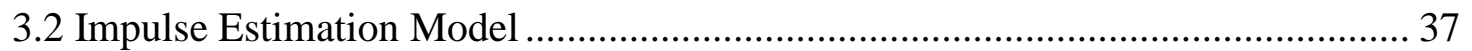

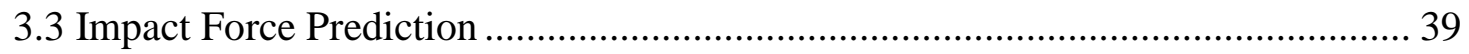

3.4 Simulation Results for Impact Force Prediction ........................................... 42

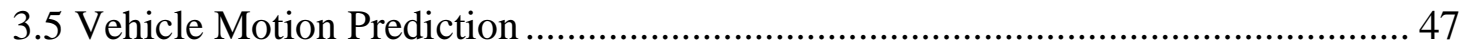

3.6 Simulation Results for Vehicle Motion Prediction ........................................ 48

CHAPTER 4 Desired Vehicle Motion Determination .................................................. 49

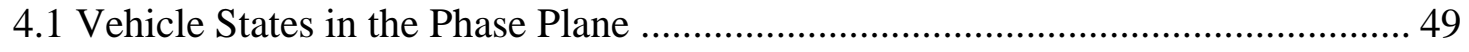

4.2 Dynamics Analysis and Beneficial Angles ................................................... 54

4.3 Vehicle Motion with Open Loop Braking Actions ........................................... 59

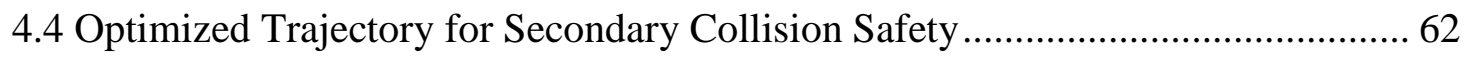

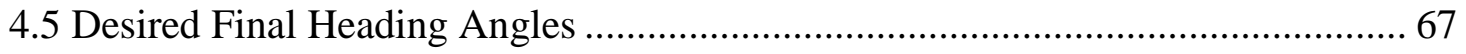

CHAPTER 5 Conclusion and Future Work................................................................... 70

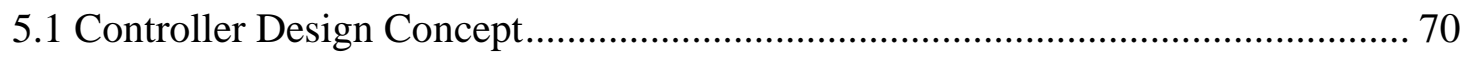

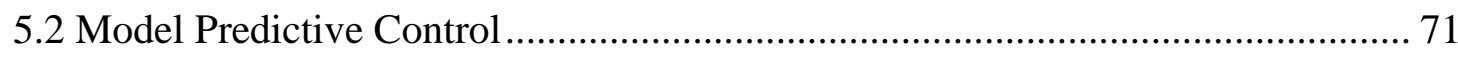

5.3 Equations for LTV-MPC Controller Design ................................................. 72

5.4 Linear Time Varying Model Predictive Control (LTV-MPC)............................ 75

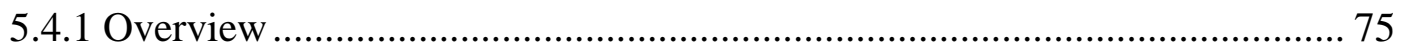

5.4.2 Linearizing at Non-Equilibrium Points ................................................... 76

5.4.3 Optimal Problem Formulation ............................................................ 78

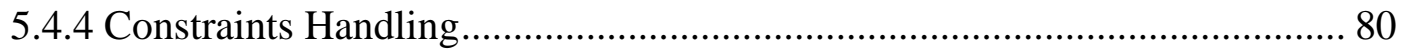

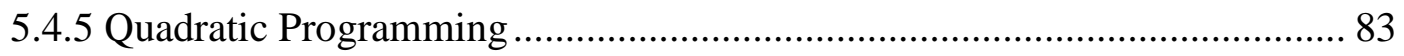

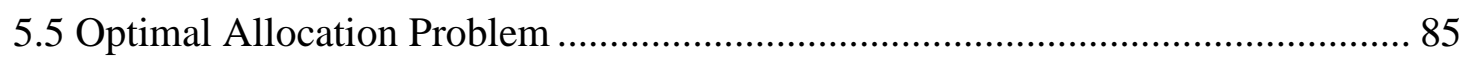

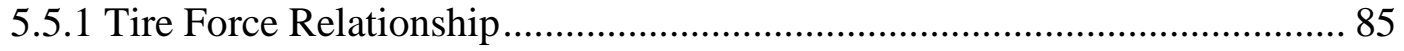




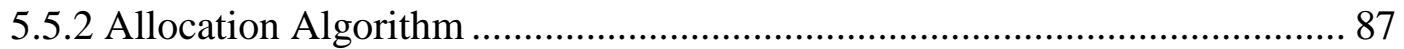

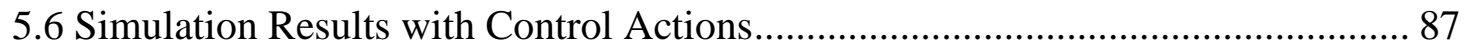

5.7 Rule-based Control for Real-time System Implementation ..................................... 91

5.7.1 Learning from the LTV-MPC Control Sequence and Rule Extraction ........ 91

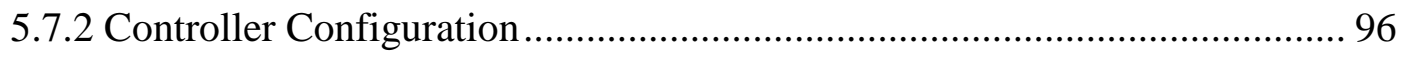

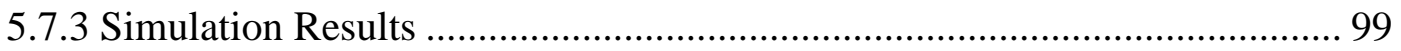

5.8 Steering for Control Enhancement ....................................................................... 104

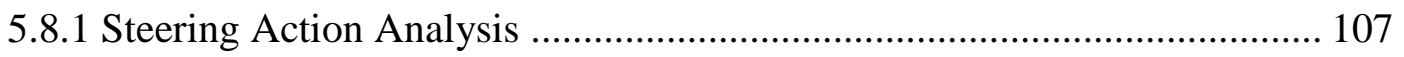

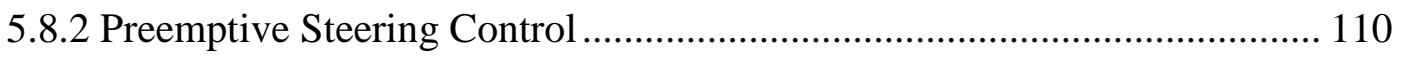

5.8.3 Preemptive Differential Braking Control.................................................. 121

CHAPTER 6 Conclusion and Future Work................................................................ 125

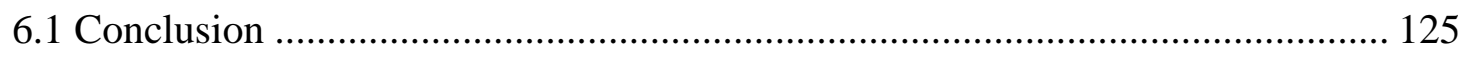

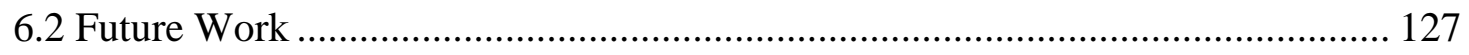

BIBIIOGRAPHY ....................................................................................................................... 128 


\section{LIST OF FIGURES}

Figure 1.1 A diagram showing how a minor first event leads to more severe secondary crashes (NASS-CDS case number: 2009-09-088 [10]) ................... 2

Figure 1.2 An imagined scenario that shows a severe secondary crash caused by an unexpected disturbance from a human-driven vehicle

Figure 1.3 Exemple secondary crash scenarios with a $90^{\circ}$ spin angle known as the most harmful secondary crash cases (The initial impact scene is not illustrated.)

Figure 1.4 Ratios of fatally injured drivers in passenger cars under two types of collisions: head on impact and side impact [15]

Figure 1.5 Vehicle trajectories of two police reported rollover crash scenarios (NASS-CDS database [10])

Figure 1.6 Range of vehicle yaw moment that can be achieved by independent brake controls, showing that the control authority decreases as side slip angle increases

Figure 1.7 An imagined secondary collision scenario caused by a first collision with a tailgating vehicle while performing AEB braking

Figure 1.8 Effective range of ESC (a) and PISC (b) on the phase plane. Each dot is an initial point of vehicle states created by an impact, and the purple dotted lines indicate the same criteria to evaluate performance. (Criteria: vehicle states within 1 second after the collision are in the specific ranges; heading angle $|\psi| \leq 25^{\circ}$, roll angle $|\phi| \leq 10^{\circ}$, lateral displacement $\left|Y_{C G}\right| \leq 1.25 \times$ lane width)

Figure 1.9 Post impact safety applications available commercially 12 
Figure 1.10 The overall procedure of estimating the collision force and predicting vehicle motion using the sensor information after the collision.

Figure 1.11 A crash scenario showing a preemptive steering control to negate the

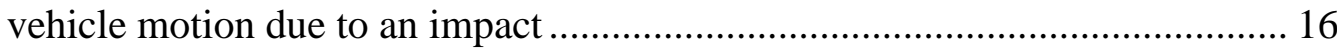

Figure 2.1 The body fixed vehicle coordinate system (ISO 8855) ........................... 17

Figure 2.2 Vehicle motion shown with both the Earth-fixed frame $X-Y$ and the

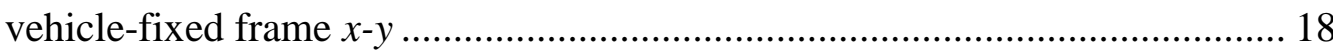

Figure 2.3 Schematic diagrams of the 4-DOF vehicle model with impact forces

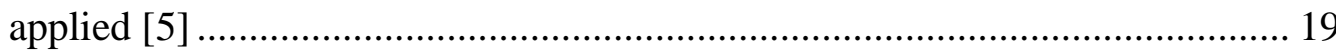

Figure 2.4 Schematic diagram of the 3-DOF vehicle model ..................................... 21

Figure 2.5 Experimental data showing combined lateral and longitudinal tire forces

Figure 2.6 Tire slip angle between a wheel centerline and the direction of travel ... 24

Figure 2.7 Longitudinal and lateral tire forces as functions of the tire slip angle and

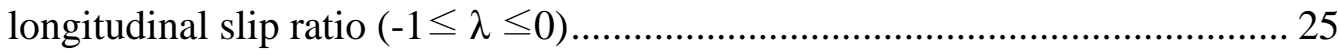

Figure 2.8 Plots to show the coupling nature of tire force generations ..................... 26

Figure 2.9 Tire Lateral Force and Slip Angle .......................................................... 27

Figure 2.10 Procedure to obtain the correction factors for the effective cornering stiffness 28

Figure 2.11 Vehicle response after an impact (No control action is involved. Initial conditions: $v_{x}=30 \mathrm{~m} / \mathrm{s}$, the impact generates $A x=3.4 \mathrm{~g}, A y=1.5 \mathrm{~g}$ ) ............. 30

Figure 2.12 Vehicle response after an impact (No control action is involved. Initial conditions: $v_{x}=30 \mathrm{~m} / \mathrm{s}$, the impact generates $A x=0.67 \mathrm{~g}, A y=0.7 \mathrm{~g}$ ) ........... 31

Figure 2.13 Vehicle response after an impact (No control action is involved. Initial conditions: $v_{x}=30 \mathrm{~m} / \mathrm{s}$, the impact generates $A x=1.7 \mathrm{~g}, A y=1.4 \mathrm{~g}$ ) ............. 32

Figure 3.1 Function diagram for impact impulse and vehicle motion prediction..... 34

Figure 3.2 An example of acceleration measurement data from the load cell on a collision test vehicle and its approximation model [59] 35 viii 
Figure 3.3 Collision time durations for 30 different car-to-car collision cases with various closing speed conditions ( $\Delta T$ : Time duration, $\Delta \mathrm{V}$ : Closing velocity) $[60,61]$ 35

Figure 3.4 Maximum force levels with different crash time durations 36

Figure 3.5 Illustration of the trapezoidal rule showing a function (black line) integration is approximated by trapezoids (red lines) ................................. 38

Figure 3.6 Impact locations on vehicle periphery in a collision [5] ....................... 39

Figure 3.7 Trianglular collision profile showing the relationship between the maximum force level and the projected impulse 40

Figure 3.8 Under-estimation and over-estimation cases depending on the actual collision time durations (Crosses indicate impulse estimation points at each timestep, and circles indicate the peak point of each profile)

Figure 3.9 Collision impulse and force estimation where the actual crash duration is 0.2 seconds while the presumed duration is 0.15 seconds. (The green bars indicate the time when the algorithm can detect the inflection point.)

Figure 3.10 Comparison of the actual and projected impulse and impact forces ('o' represents the projection point of the maximum forces at $\Delta T=0.15$ seconds)

Figure 3.11 Collision prediction in a case when the actual collision time duration ( $\Delta T=0.2$ seconds) is longer than the nominal value 43

Figure 3.12 Collision prediction in a case when the actual collision time duration $(\Delta T=0.1$ seconds $)$ is shorter than the nominal value................................ 44

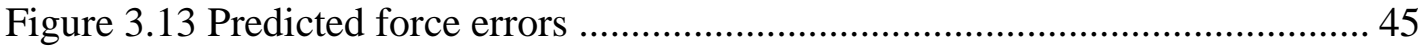

Figure 3.14 An oblique frontal offset crash test scene in the NHTSA Crash Test [62]

Figure 3.15 Validation of the prediction algorithm using a real crash data ('o' represents the projection point of the maximum forces, the gray lines represents the triangular shape of the predicted forces) 46 
Figure 3.16 Block diagram of the collision impact estimation and the motion prediction model

Figure 3.17 Vehicle motion prediction results with different durations of collision forces. 48

Figure 4.1 Vehicle dynamic motion analysis using the yaw rate-sideslip angle phase plane plot. Blue lines: converged in 1 second, Red lines: not converged in 1 second (Convergence criteria: $\omega_{z} \in\left[-3.0^{\circ} / \mathrm{s},+3.0^{\circ} / \mathrm{s}\right], \beta \in\left[-1.5^{\circ},+1.5^{\circ}\right]$ ) 50

Figure 4.2 A few imagined scenarios with various impact conditions that will create different initial conditions on the phase plane 51

Figure 4.3 Extended phase plane. Region (1) is the convergence region for the origin, while Region (2) represents the one for an alternative equilibrium. Point ' $A$ ' indicates a possible initial state after an impact outside of region (1). Blue line illustrates the resulting vehicle motion without control

Figure 4.4 A sequential scene captured on dashboard camera in the striking car, which ends with a side pole crash. The sequence is from upper left to lower right [70]

Figure 4.5 A recovery motion of a fleeing vehicle chased by a law enforcement vehicle executing a PIT (Precision Immobilization Technique) maneuver. The sequence is from upper left to lower right. (Blue color: The suspect vehicle, Black-and-white color: The police vehicle) [71] ....................................... 55

Figure 4.6 Induced yaw moment from the lateral tire forces .................................56

Figure 4.7 Open-loop simulation results showing the vehicle motion, forces, and yaw moment generated by the tires

Figure 4.8 Tire lateral forces and slip angle after an impact ................................ 58

Figure 4.9 Schematic view at (A), (B), and (C) time sequence in Figure 4.8 .......... 58

Figure 4.10 Projected vehicle position and motion without any control ................. 59 
Figure 4.11 Comparison of braking actions with the initial state $v_{x}=30 \mathrm{~m} / \mathrm{s}$ and $\omega_{z}=100 \%$ s. (a) 4-wheel brake lock. (b) Rear 2-wheel brakes lock. (c) Front 2wheel brake lock.

Figure 4.12 Reduction in tire lateral force when braking is applied $\left(i \in f_{L}, f_{R}, r_{L}, r_{R}\right) 61$ Figure 4.13 Effects of vehicle yaw moment when the lateral tire forces are affected by braking 62

Figure 4.14 Vehicle trajectories with different objective functions .......................6 64

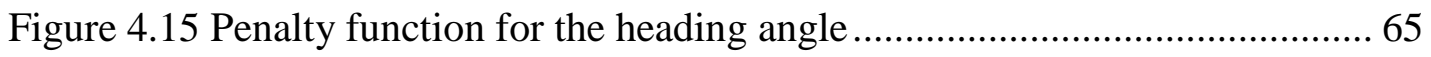

Figure 4.16 Optimized vehicle trajectory for two different weightings ................... 66

Figure 4.17 Optimization results showing desired final heading angles for various initial yaw rates (Initial conditions: $V X=30 \mathrm{~m} / \mathrm{s}, V Y=2 \mathrm{~m} / \mathrm{s}$ ) ................... 68

Figure 4.18 Desired final heading angle maps depending on vehicle speed ............ 69

Figure 5.1 Hierarchical frame work for the vehicle control systems....................... 70

Figure 5.2 Flow chart of the control methodology ............................................. 74

Figure 5.3 Architecture of the proposed control system ..................................... 75

Figure 5.4 Tire force vectors, showing the force effects on vehicle yaw moment with

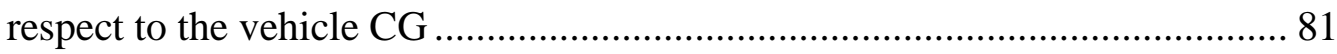

Figure 5.5 Vehicle yaw moments that can be generated by braking at zero steering angle. The shaded area between red line (upper bound) and blue dashed line (lower bound) depicts achievable region for all possible slip ratio $(-1 \leq \lambda \leq 0)$

Figure 5.6 Effects of front steering on vehicle yaw moment by braking. Only the front wheels are shown because the rear wheels are not steered.

Figure 5.7 Upper and lower bounds of available vehicle lateral forces in the earthfixed frame 83

Figure 5.8 Signal flows for the optimal allocation algorithm 85

Figure 5.9 Vehicle trajectories under three control strategies. (Vehicle sizes are doubled.) 88 
Figure 5.10 Virtual control inputs from the proposed control strategy. Feasible boundaries are shown by gray shaded regions. Virtual controls solved by MPC are shown in bold lines. 88

Figure 5.11 Longitudinal tire slip ratio commands and corresponding longitudinal forces calculated by the optimal allocation problem. The gray shaded regions depict the feasible control bounds

Figure 5.12 Vehicle trajectories under several initial conditions representing different levels of impact: yaw rate 80 200\% s and heading angle 9 23 with fixed lateral speed $(5 \mathrm{~m} / \mathrm{s})$ and longitudinal speed $(30 \mathrm{~m} / \mathrm{s})$ 90

Figure 5.13 Vehicle motion trajectories and brake control sequences from the LTVMPC (Final heading angle: $360^{\circ}$ ) 92

Figure 5.14 Vehicle motion trajectories and brake control sequences from the LTVMPC (Final heading angle: $180^{\circ}$ ). 93

Figure 5.15 Vehicle motion trajectories and brake control sequences from the LTVMPC (Final heading angle: $540^{\circ}$ )

Figure 5.16 Vehicle dynamic motion with brake controls (Zoomed in from Figure 95

Figure 5.17 Switching structure for the rule-based control 96

Figure 5.18 Simulation result comparison between the rule-based control and LTVMPC 100

Figure 5.19 Vehicle dynamics parameters when control actions initiated ............. 101

Figure 5.20 Vehicle trajectory comparisons .................................................. 102

Figure 5.21 Simulation results of 17 different cases......................................... 103

Figure 5.22 Generating yaw moment by using differential braking and steering .. 104 Figure 5.23 Vehicle dynamics showing the steering changes the direction of the front tire forces and yaw moment to the vehicle CG. (a) Vehicle model showing the tire force, slip angle, and steering angle. (b) Possible yaw moments that can be generated by each tire. Red circles indicate $0^{\circ}$ steering, the blue stars 
indicate $-10^{\circ}$ steering. Gray lines are yaw moment with a tire slip ratio between $\lambda=0$ (no braking) and $\lambda=-1$ (wheel locking). 106

Figure 5.24 Open loop dynamics on the phase plane at $v_{x}=30 \mathrm{~m} / \mathrm{s}$ with different steering angles (Black bold lines: vehicle trajectories from the origin for 1 second, magenta dashed line indicates peak level of yaw rate from the origin)

Figure 5.25 Open loop dynamics on the phase plane with $\delta=-5^{\circ}$ at different vehicle speed conditions (Black bold lines: vehicle trajectories from the origin for 1 second, magenta dashed line indicates peak level of yaw rate).....

Figure 5.26 Yaw rate responses with a same step steering input under different longitudinal speeds.

Figure 5.27 A crash scenario showing a counter-steering control to negate the vehicle motion due to an impact (Shaded triangle regions illustrate possible sensor coverage for detecting an approaching vehicle to the host vehicle) .... 111

Figure 5.28 Vehicle motions on the phase plane at $v_{x}=30 \mathrm{~m} / \mathrm{s}$ showing the advantage of preemptive steering action. The vehicle motion with an impact condition without any steering action, which causes $\Delta \omega_{z}=50^{\circ} / s, \Delta \beta=-3^{\circ}$, is shown in (a), and the vehicle motion with a preemptive steering $\left(-2.5^{\circ}\right)$ prior to the same impact is shown in (b) 112

Figure 5.29 Block diagram of the control structure, $\underline{\xi}=\left[v_{y} \omega_{z}\right]^{T} \ldots \ldots \ldots \ldots \ldots \ldots \ldots \ldots . . . . . . . .112$

Figure 5.30 Structure for the Feed-forward steering control ............................. 114

Figure 5.31 Trajectories of the vehicles with and without the proposed preemptive

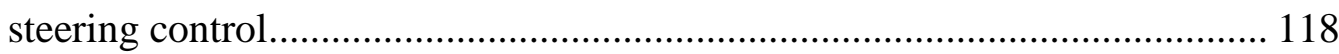

Figure 5.32 Test result comparison. (vehicle motion without control, with a steering and brake control during and after the impact (PISC), and with preemptive counter-steering control before the impact ) 
Figure 5.33 Evolution of the vehicle motion trajectories on the slip angle-yaw rate phase plane $\left(\beta-\omega_{z}\right)$ after an impact ........................................................... 120

Figure 5.34 Maximum lateral deviation and maximum yaw rate comparison with and without the preemptive steering control:.

Figure 5.35 Yaw moment that can be generated by each tire (Orange circles and arrows illustrate the change of yaw moment with braking at zero slip angle. Gray lines are yaw moment in $-1<\lambda<0$.)

Figure 5.36 Illustration of the preemptive differential brake function applying the front and rear right tire brakes to generate vehicle yaw moment ................... 122

Figure 5.37 Preemptive differential brake test result ........................................... 124

Figure 5.38 Trajectories of the vehicles with the preemptive differential brake function 


\section{LIST OF TABLES}

Table 2.1 Vehicle parameters for the 4-DOF model............................................ 29

Table 3.1 Area errors of the collision force prediction algorithm ......................... 46

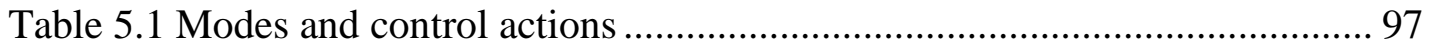

Table 5.2 Rule-based mode switching logic ..................................................... 98

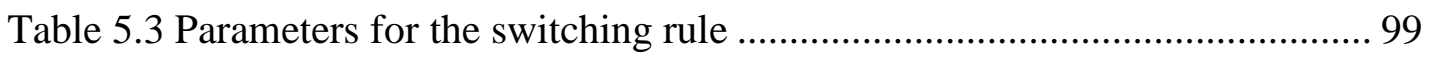

Table 5.4 Reference conditions for collision forces .......................................... 100

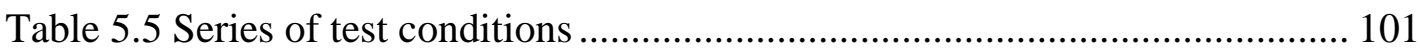




\section{LIST OF SYMBOLS}

$a, b \quad$ Distance from the axles to the vehicle CG (m)

$A_{x}, A_{y} \quad$ Longitudinal and lateral acceleration at vehicle CG $\left(\mathrm{m} / \mathrm{s}^{2}\right)$

$C_{\alpha} \quad$ Cornering stiffness of a tire (N/rad)

$C_{f}, C_{r} \quad$ Cornering stiffness of tires at the front axle and rear axle (N/rad)

$D_{s} \quad$ Total suspension roll damping coefficient $(\mathrm{N} \cdot \mathrm{m} \cdot \mathrm{s} / \mathrm{rad})$

$F_{x}, F_{y} \quad$ Longitudinal and lateral forces $(\mathrm{N})$

$F_{x, f,} F_{x, r} \quad$ Longitudinal tire forces at the front axle and rear axle $(\mathrm{N})$,

$F_{y, f}, F_{y, r} \quad$ Lateral tire forces at the front axle and the rear axle (N)

$F_{z} \quad$ Wheel vertical load (N)

$g \quad$ Gravitational acceleration $\left(\mathrm{m} / \mathrm{s}^{2}\right)$

$h \quad$ Distance from the sprung mass CG to the roll axis (m)

$h_{C G} \quad$ Vehicle CG height above the ground (m)

$I_{x x s} \quad$ Sprung mass roll moment of inertia about the roll axis $\left(\mathrm{kg} \cdot \mathrm{m}^{2}\right)$

$I_{x z} \quad$ Sprung mass product of inertia about the roll and yaw axes $\left(\mathrm{kg} \cdot \mathrm{m}^{2}\right)$

$I_{z z} \quad$ Vehicle yaw moment of inertia $\left(\mathrm{kg} \cdot \mathrm{m}^{2}\right)$

$K_{s} \quad$ Total suspension roll stiffness $(\mathrm{N} \cdot \mathrm{m} / \mathrm{rad})$

$K_{s, f,} K_{s, r} \quad$ Roll stiffness of front and rear suspensions $(\mathrm{N} \cdot \mathrm{m} / \mathrm{rad})$

$L \quad$ Wheelbase (m)

$m \quad$ Vehicle mass $(\mathrm{kg})$

$m_{R} \quad$ Rolling (sprung) mass $(\mathrm{kg})$

$m_{N R} \quad$ Non-rolling (unsprung) mass ( $\mathrm{kg}$ )

$M_{z} \quad$ Yaw moment $(\mathrm{N} \cdot \mathrm{m})$

$n \quad$ Prediction window length (-) 


\begin{tabular}{|c|c|}
\hline$P_{b}$ & Braking pressure of the wheel cylinder (bar) \\
\hline$\Delta P_{x,} \Delta P_{y}$ & Collision impulses decomposed to the $x-y$ directions $(\mathrm{N} \cdot \mathrm{s})$ \\
\hline$Q$ & Optimization weighting matrix for the states vectors (-) \\
\hline$R$ & Optimization weighting matrix for the inputs vectors (-) \\
\hline$S$ & Sliding surface function \\
\hline$T_{W}$ & Track width of the vehicle $(\mathrm{m})$ \\
\hline$\Delta T$ & Collision time duration (seconds) \\
\hline$\Delta t$ & Sampling time (seconds) \\
\hline$u$ & Actual control input matrix consisting of wheel brake forces \\
\hline$v$ & Virtual control input matrix consisting of forces and moment \\
\hline$v_{x}, v_{y}$ & Vehicle longitudinal, lateral velocity in the body fixed coordinate $(\mathrm{m} / \mathrm{s})$ \\
\hline$V_{X}, V_{Y}$ & $\begin{array}{l}\text { Vehicle longitudinal, lateral velocity in the earth-fixed frame } \\
\text { coordinate }(\mathrm{m} / \mathrm{s})\end{array}$ \\
\hline$x, y, z$ & Vehicle body fixed coordinate at the vehicle CG \\
\hline$x_{A}, y_{A}, z_{A}$ & Location of the vehicle impact (m) \\
\hline$X, Y$ & Earth-fixed frame coordinate \\
\hline$Y_{C G}$ & Lateral displacement of the vehicle CG (m) \\
\hline$\alpha$ & Wheel sideslip angle (rad) \\
\hline$\beta$ & Vehicle slip angle (rad) \\
\hline$\delta$ & Road wheel steering angle (rad) \\
\hline$\theta$ & Orientation angle of the vehicle before impact (rad) \\
\hline$\lambda$ & Wheel slip ratio (-) \\
\hline$\mu$ & Friction coefficient (-) \\
\hline$\xi$ & Vehicle states in the state space equation \\
\hline$\psi$ & Vehicle yaw angle (rad) \\
\hline$\omega_{x}$ & Roll rate $(\mathrm{rad} / \mathrm{s})$ \\
\hline$\omega_{z}$ & Yaw rate $(\mathrm{rad} / \mathrm{s})$ \\
\hline
\end{tabular}




\section{ABSTRACT}

Statistical data of road traffic fatalities show that fatalities in multiple-event crashes are higher than in single-event crashes. Most vehicle safety systems were developed to mitigate first crash events. Few active safety systems can deal with subsequent crash events. After a first crash event, drivers may not react in a timely or correct manner, which can have devastating consequences. Production active safety systems such as Electronic Stability Control (ESC) may not react to a first crash event properly unless such events are within their design specifications. The goal of this thesis is to propose control strategies that bring the vehicle state back to regions where drivers and ESC can easily take over the control, so that the severity of possible subsequent (secondary) crashes can be reduced. Because the most contributing causes of fatal secondary crashes are large lateral deviations and heading angle changes, the proposed algorithms consider both lateral displacement and heading of the vehicle. To characterize the vehicle motion after a crash event, a collision force estimation method and a vehicle motion prediction scheme are proposed. The model-based algorithm uses sensing information from the early stage of a collision process, so that the collision force can be predicted and the desired vehicle state can be determined promptly. The final heading angles are determined off-line and results are stored in a look-up table for faster implementation. Linear Time Varying Model Predictive Control (LTV-MPC) method is used to obtain the control signals, with the key tire nonlinearities captured through linearization. This algorithm considers tire force constraints based on the combined-slip tire model. The computed high-level control signals are realized through a control allocation problem which maps vehicle motion commands to tire braking forces considering constraints. For faster real-time implementation, a rule-based control strategy is obtained by observing the LTV-MPC control behaviors. Several rules were constructed, 
and the obtained vehicle motions under the rule-based control are similar to those under the optimal control method while avoiding heavy on-board computations. Lastly, this thesis proposes a preemptive steering control concept. By assessing the expected strength of an imminent collision force from another vehicle, a preemptive steering control is applied to mitigate the imminent impact. The effectiveness of proposed algorithms is demonstrated by simulations. 


\section{CHAPTER 1 INTRODUCTION}

\subsection{Motivation}

Approximately 5.6 million motor vehicle crashes were reported during 2012 in the United States, of which about 30,000 were fatal crashes [1]. Among the fatal crashes, about $40 \%$ were the results of multiple vehicle crashes. Several statistical studies based on National Automotive Sampling System - Crashworthiness Data System (NASS-CDS) data [2-4] indicate that number of multiple impact crashes has been increasing. These reports found that the risk of severe injuries is much higher in multiple impact crashes than in single impact crashes. In [5], it is also pointed out that the risks of both injuries and fatalities increased with the number of collision events. Moreover, harmful subsequent impact crashes are often associated with high-speed crashes, as shown in [6], because the vehicle's kinetic energy is relatively high and the vehicle is more likely to sustain a subsequent impact after the first event.

Typically, drivers pay more attention to the front than to the side or the back while driving. As drivers have more information from the instrument cluster, GPS map guidance, and/or head-up display (HUD), remaining vigilant for possible events from the side or the back is harder. According to the statistical data presented in [7], continuous monitoring of the surroundings while maintaining the lane position is not an easy task for average drivers. Furthermore, the risk increases if a driver is distracted, such as by eating, drinking, or talking on the phone. For this reason, drivers are not always ready to react to unexpected motions from other vehicles. In these situations, drivers can easily panic or freeze after an unexpected minor collision at the side or back of the vehicle, and can easily lose control. 


\subsubsection{Risk of Lateral Deviation}

According to the collision test results in [8], which review the leg movement on the brake pedal, the driver's foot is likely to be lifted from the brake pedal within a short time during and after a crash. Accordingly, wheels will roll freely and the vehicle rotates due to the impact. Until the driver is able to resume control (putting the foot back on the brake pedal), the vehicle may have built up large lateral deviation and moved to another lane. If the vehicle intrudes into an opposing traffic lane, the secondary impact can be devastating.

The analysis of vehicle dynamic motion after an impact in [9] indicates that vehicle kinetic energy and lateral lane deviation after an initial impact play important roles in the risk of the secondary impact. Vehicle-to-vehicle collisions create impulsive disturbance forces and cause significant changes in heading angle and lateral position. For example, depending on the location relative to the vehicle center of gravity, the vehicle yaw rate immediately after the impact can be quite different. In addition, the vehicle lateral acceleration, due to the lateral component of the impact vector, introduces side slip velocity. Therefore, both the vehicle heading angle and the velocity direction can change significantly by the impact, which means the vehicle can travel on a very different path. As a real world example, Figure 1.1 shows a police-reported traffic crash report, found in the NASS-CDS database, which depicts devastating multi-impact consequences after a minor initial impact. This multi-event accident occurred on a divided highway with a median strip barrier and four straight lanes. The road was dry and the weather was clear and sunny.

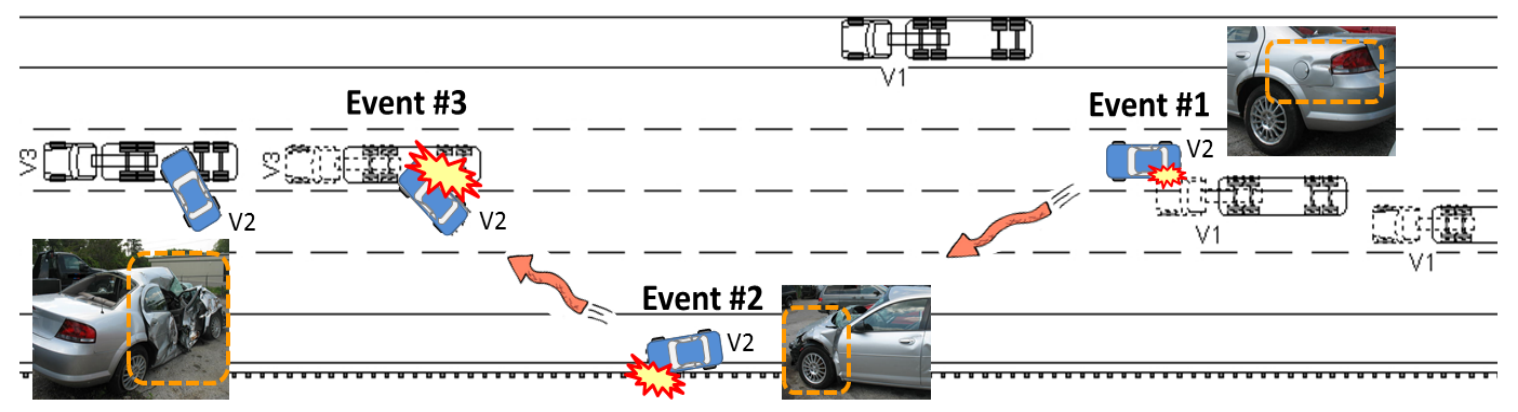

Figure 1.1 A diagram showing how a minor first event leads to more severe secondary crashes (NASS-CDS case number: 2009-09-088 [10]) 
The three vehicles involved were traveling in the same direction before the crash. Vehicle 1 (V1) and Vehicle 3 (V3) illustrate different trucks, and Vehicle 2 (V2) is a passenger car which had multiple impacts. Vehicles depicted with dotted lines represent moving state and vehicles with solid lines represent stopped state.

The first event started when V1 changed lanes. The driver of V1 might not have realized the existence of $\mathrm{V} 2$, and the right front corner of V1 came in contact with the left rear corner of V2. Then, V2 spun counter-clockwise and began crossing lanes to the left until V2's left front side came in contact with a metal guardrail, causing Event 2. After that, V2 spun clockwise and crossed lanes to the right. Finally, in Event 3, V2's right side struck the left rear tires of V3's trailer. The passenger in V2 suffered severe injuries. As imagined from the picture at each event, the first impact for V2 was relatively minor structural deformation, and severities were much higher in subsequent impacts.

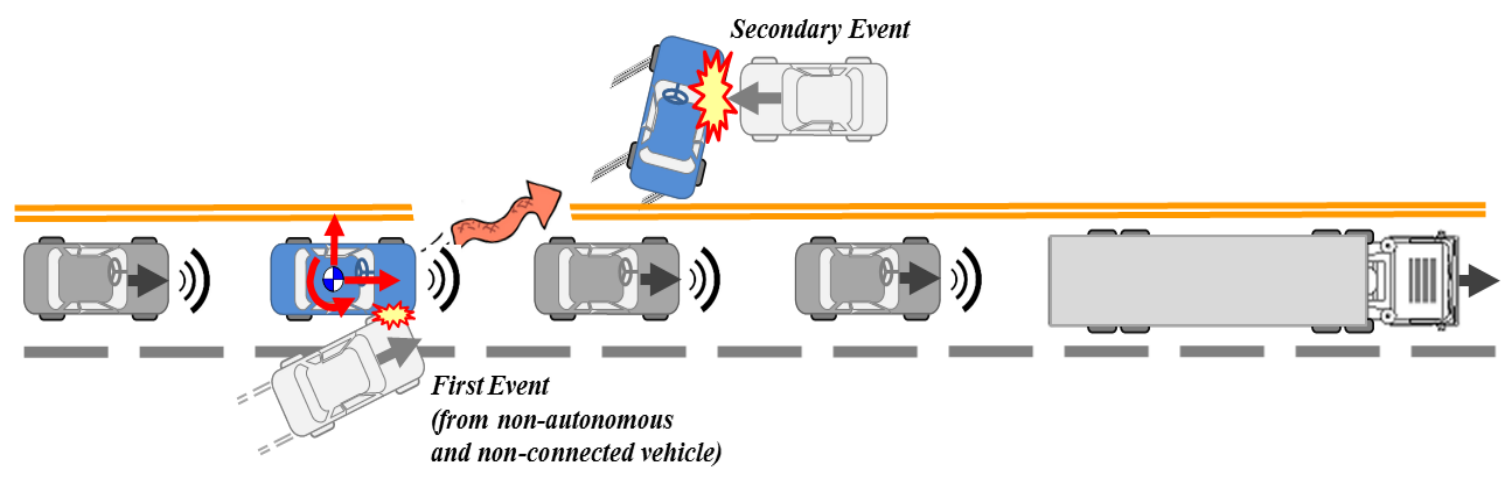

Figure 1.2 An imagined scenario that shows a severe secondary crash caused by an unexpected disturbance from a human-driven vehicle

Similar multiple impact scenarios can occur even for automated vehicles in the future, as shown in Figure 1.2. Starting from one of the earlier automated highway system studies by the California PATH program [11], the majority of these projects have focused on the performance of 'self-driving' cars and cooperation among autonomous cars. Many of the prototype autonomous vehicles were designed without considering the disturbance from impacts. Judging from the recent autonomous vehicle legislation enacted in a few states including California [12], the driver is still expected to be prepared for emergency events. Because the driver in an autonomous car is less likely to be prepared to react in a post-crash event properly due to the prolonged disengagement in the driving task, an automatic function for post-impact control is even more needed. As 
long as human-driven vehicles continue to be present on public roads, a countermeasure to mitigate a secondary collision is needed.

\subsubsection{Risk of Vehicle Rotation and Side Impact Crashes}

The National Highway Traffic Safety Administration (NHTSA) crash analysis report [6] based on data from 1988 to 2004 shows the vehicle spin angle distribution in the most harmful secondary event crashes. It shows that secondary events with turning angles of around $90^{\circ}$ (either clockwise or counter-clockwise) cause the most harmful secondary crashes. Examples of side impacts with another moving vehicle or a fixed roadside object are shown in Figure 1.3. Based on injury scale level data, as shown in [13], side impacts in a secondary event impose higher risk for the occupants. Since the sides of vehicles have less crash-energy absorbing structures than the front and rear sections, the risk of fatalities and serious injuries with side crashes is higher [14].

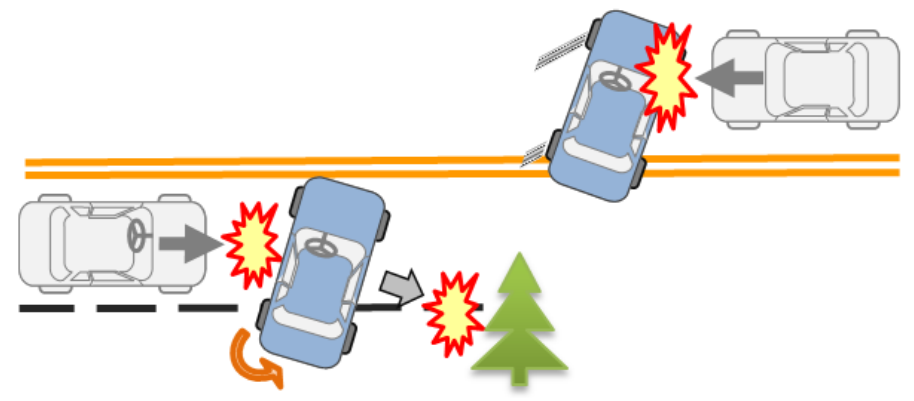

Figure 1.3 Exemple secondary crash scenarios with a $90^{\circ}$ spin angle known as the most harmful secondary crash cases (The initial impact scene is not illustrated.)

An investigation of collision severities related to the part (or structure) of the vehicle being struck is conducted in [15]. This study shows the importance of side impact severity by comparing different types of collisions and striking cars. It also emphasizes the high risk of side impacts by showing driver fatality ratios among types of crashes based on the Fatal Analysis Reporting System (FARS) database. As shown in Figure 1.4, it is obvious that the number of fatalities in the struck vehicle is higher when the striking vehicle is larger and heavier, and the consequences of crashes tend to be more severe in side impact cases than head-on collision cases. 


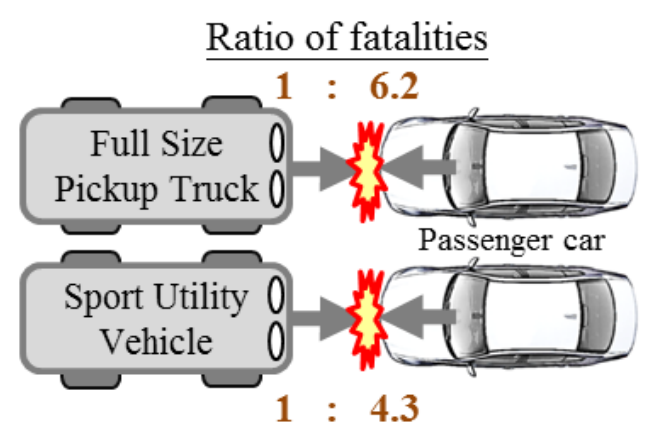

(a) Head on collisions

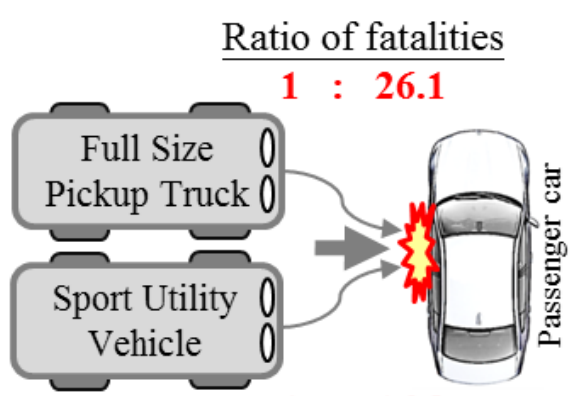

$1: 16.3$

Figure 1.4 Ratios of fatally injured drivers in passenger cars under two types of collisions: head on impact and side impact [15]

One reason for this result is that the most common areas for occupant injury in the side-struck vehicle are the chest and the head, which cause higher fatalities. Because the standard 3-point seat belts in the car cannot securely hold the lateral movement of the upper body caused by side impacts, the driver's head and chest can strike the vehicle frame or window [16]. Besides, because there is not enough space between the occupant and the side of the vehicle, the passenger compartment is vulnerable to door intrusion [17]. Due to this risk, many safety improvements have been made by reinforcing the structure design of the side doors, improving restraint systems, and adding side air bags. However, these safety protections can be activated only once, and may not be effective in multiple collision cases.

$90^{\circ}$ vehicle rotation can cause another secondary crash type-rollover. According to a crash injury study in the United Kingdom [18], the number of rollovers as a secondary event is higher than rollovers as a first event. In fact, the injury risks for the former scenario (an initial crash with another vehicle, then rollover) is about 1.5 times higher than the latter scenario [19]. NASS-CDS accident examples, as shown in Figure 1.5 , illustrate these two types of rollover cases. In rollover as a secondary event case (a), the initial collision with another car changes the vehicle orientation and leads a loss of control. Rollover as a first event case (b) usually happens with tire saturations due to excessive steering from the driver, who then fails to maintain control [20]. It should be noted that many rollover accidents are caused by an initial impact with another vehicle leading to severe secondary collision scenarios involving a large heading angle. 


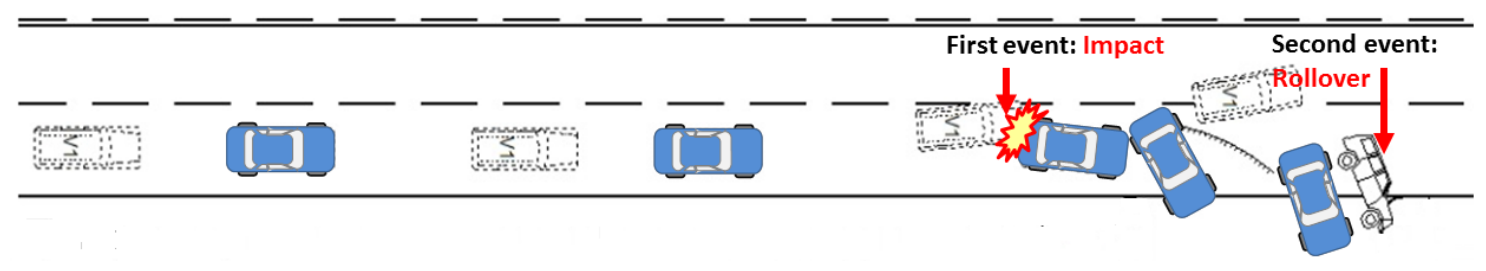

(a) Rollover as a secondary event (NASS-CDS case number: 2012-76-159)

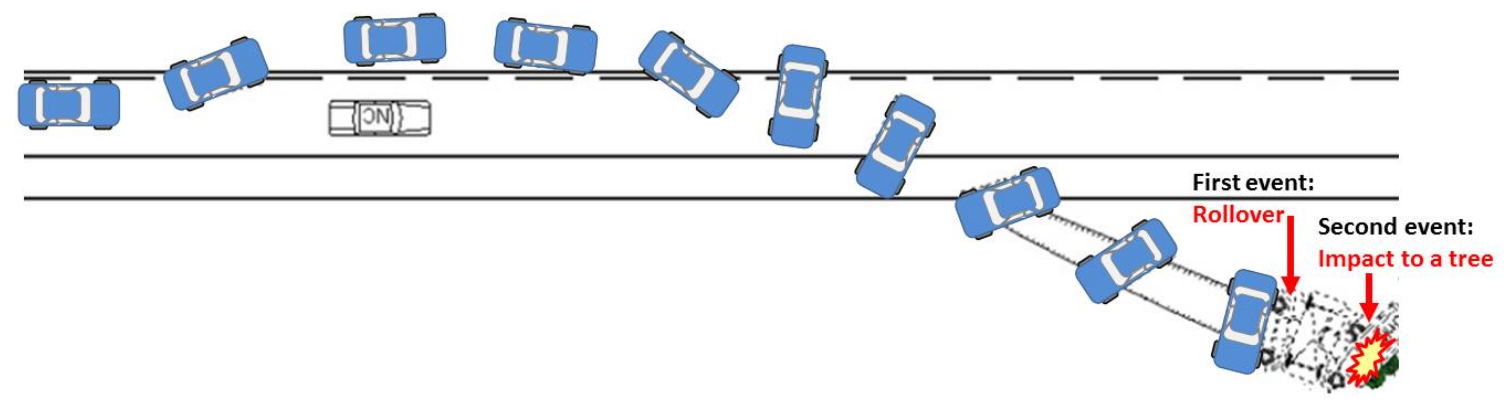

(b) Rollover as a first event (NASS-CDS case number: 2012-13-024)

Figure 1.5 Vehicle trajectories of two police reported rollover crash scenarios (NASSCDS database [10])

Obviously, the severities of these crashes are higher as the vehicle speed becomes higher [21].

\subsection{Vehicle Safety Systems}

Various vehicle safety systems have been developed to prevent and reduce vehicle crashes. Safety systems are usually categorized as either 'Passive Safety' or 'Active Safety': Passive safety systems are reactive measures to reduce the severity of occupants' injuries once an accident happened. Common examples of these are seatbelts, airbags, and crumple zones of the vehicle structures. On the contrary, active safety functions are preemptive (or preventative) measures activated before the collision to avoid or reduce the probability and/or severity of crashes. ABS (Anti-locking Braking System), ESC (Electronic Stability Control), collision warning or mitigations systems, and lane departure warning or assistance system are representative active safety systems.

Among these active safety systems, ESC has been found to be especially effective [22-27]. ESC detects tire skidding and loss of steering control, then it automatically applies wheel brakes independently to reduce side slip or to follow the desired yaw rate. 
It was found that vehicles with ESC have significantly lower single-vehicle crash risk in passenger cars and sports utility vehicles (SUV) than those without ESC. However, while it has had positive effects in reducing single vehicle crashes, it has limited effect on multiple-vehicle and secondary crashes [25]. Because the control algorithms of ESC systems were mainly designed to prevent spinning or skidding, the controller mainly considers the deviation between the measured vehicle yaw rate and the desired yaw rate determined by a vehicle model. For this reason, when an impact occurs, some ESC systems could even misinterpret the situation as a sensor failure and could deactivate.

Furthermore, since the tire force is saturated at large tire slip angles, controlling the vehicle under large side slip angle is challenging [28]. This is also one of the reasons why ESC systems aim to maintain the tire slip angles within a small range [29]. In other words, a relatively large external disturbance (e.g. due to a vehicle-to-vehicle collision), which causes a high side slip angle, may not be within the scope of a conventional ESC. In a severe crash, a vehicle can skid and the vehicle sideslip angle can be larger than 45 degrees. In this situation, the wheel brake is ineffective and the ability to control yaw rate is lost. Figure 1.6 illustrates the problem of large vehicle side slip angle for regulating vehicle yaw rate with independent braking control in ESC. This figure is obtained by solving the kinematic equation for yaw moment. Since the locations of the tires are different with respect to the center of gravity (CG), the roles of each tire in generating vehicle yaw moment are different.

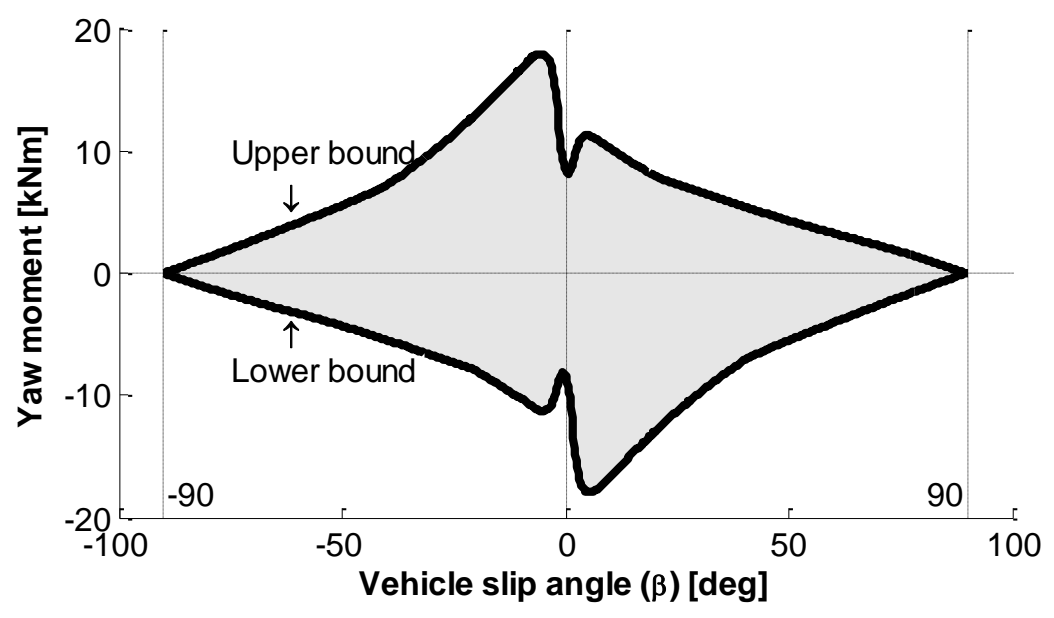

Figure 1.6 Range of vehicle yaw moment that can be achieved by independent brake controls, showing that the control authority decreases as side slip angle increases 
Detailed derivation and analysis on this will be addressed in Section 5.4.4. One important note for this figure is that the controllable range of the vehicle yaw moment (control authority) decreases as the side slip angle of the vehicle increases.

In a situation when a significant sideslip angle is developed due to a collision, the available magnitude of yaw moment control is lower than when the sideslip angle is small. Hence, the control authority will be smaller than in typical ESC operation conditions (sideslip angle is maintained within \pm 5 degrees [30, 31]). Moreover, the reduced control authority may result in a large lateral deviation, which may also increase the risk of secondary collisions. In addition, if the driver's steering action does not effectively reduce the vehicle slip angle during an initial impact, today's production ESCs may not be able to effectively handle the vehicle motion to prevent secondary impacts. Although the driver's steering action in normal situations is an important indication of the desired vehicle direction, this may not be true immediately after a collision because of the possibility that the driver's action could be inappropriate. Typically, drivers may be startled due to the impact and then fail to maintain proper control; thus hindering ESC from performing properly to avoid further collisions [32].

Secondary collision can happen when a vehicle performs an emergency braking while the vehicle is followed by tailgating traffics. Tailgating can happen when drivers are not conscious of risk of driving closely behind a leading vehicle. In this situation, if the leading vehicle decelerates suddenly to avoid colliding with its lead vehicle, it has a high risk of causing a rear-end collision [33]. Similarly, a vehicle with Autonomous Emergency Braking (AEB) system also risks from being struck behind. AEB is an active safety system which supports the driver by applying heavy brake when an imminent forward collision is detected [34, 35]. However, although the AEB function helps to mitigate a forward collision, it may increase the risk of struck from behind. An example scenario is illustrated in Figure 1.7 showing that a vehicle with AEB performs braking to dodge the danger to the front, but it also causes a collision with another vehicle from the back. If the vehicle speed is high, the first impact may result in an undesirable vehicle motion that leads to a secondary crash. 


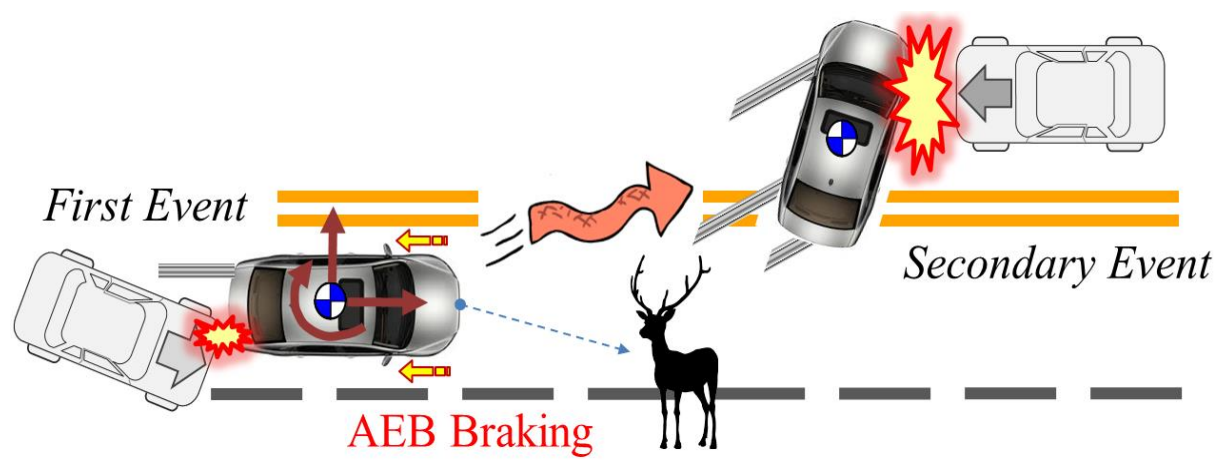

Figure 1.7 An imagined secondary collision scenario caused by a first collision with a tailgating vehicle while performing AEB braking

\subsection{Controls to Avoid Secondary Collisions}

To enhance vehicle safety in secondary collisions, an algorithm for collision disturbance detection and control countermeasures is proposed in [5]. The proposed system, Post-Impact Stability Control (PISC), was found to effectively attenuate the vehicle yaw rate and sideslip after an impact. Its objective was to mitigate the initial vehicle yaw and side slip motion at the early stage of the collision and then to hand over the vehicle control back to the driver and the "regular ESC system." Figure 1.8 shows the range of effective intervention by ESC and PISC, which shows that PISC has a higher effective region than ESC. However, as shown at the lower right corner of Figure 1.8 (b), the PISC still shows a limitation: large lateral deviation and/or heading angle. The vehicle may drift to a neighboring lane and be involved in a secondary crash, which we had shown to be risky.

To reduce the vehicle motion after an impact, automatic full braking was devised by BOSCH [36]. The proposed system is called Secondary Collision Mitigation (SCM), which is triggered by the airbag sensor (accelerometer), and four-wheel brake pressure is built by ESC to perform an automatic deceleration. Since the kinetic energy is reduced by the braking, it is hoped that SCM is capable of reducing the severity of potential secondary collisions [32]. An experimental feasibility test [37] showed that there are cases where the test driver could not move their foot from the accelerator to the brake pedal during a crash. In some other cases, the accelerator and the brake pedals were 
pressed simultaneously. They emphasized that the abnormal vehicle motion after an initial collision is a challenging situation even for experienced drivers, and automatic full deceleration with the braking function can reduce the risk of secondary collisions.

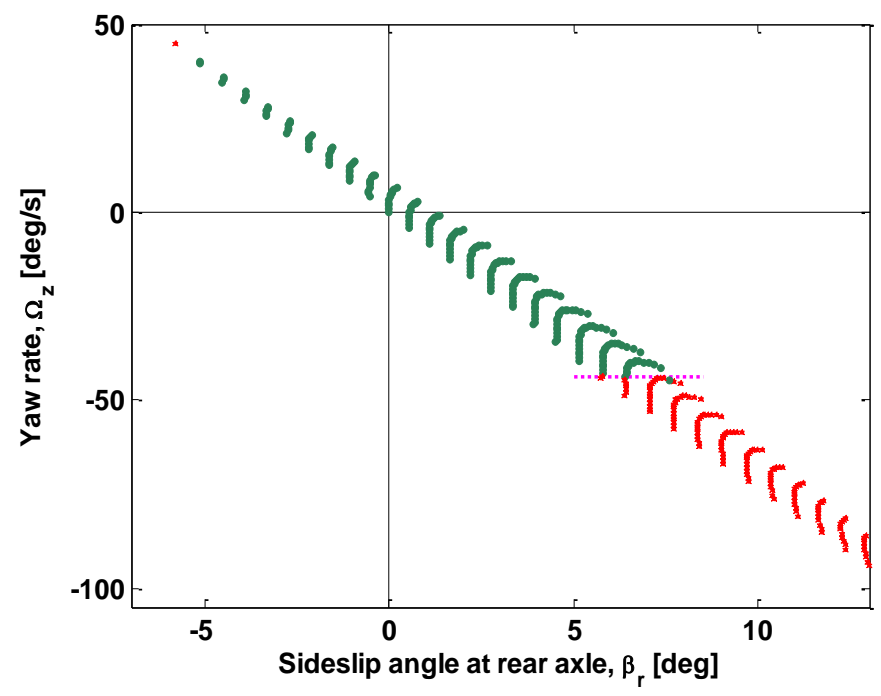

(a)

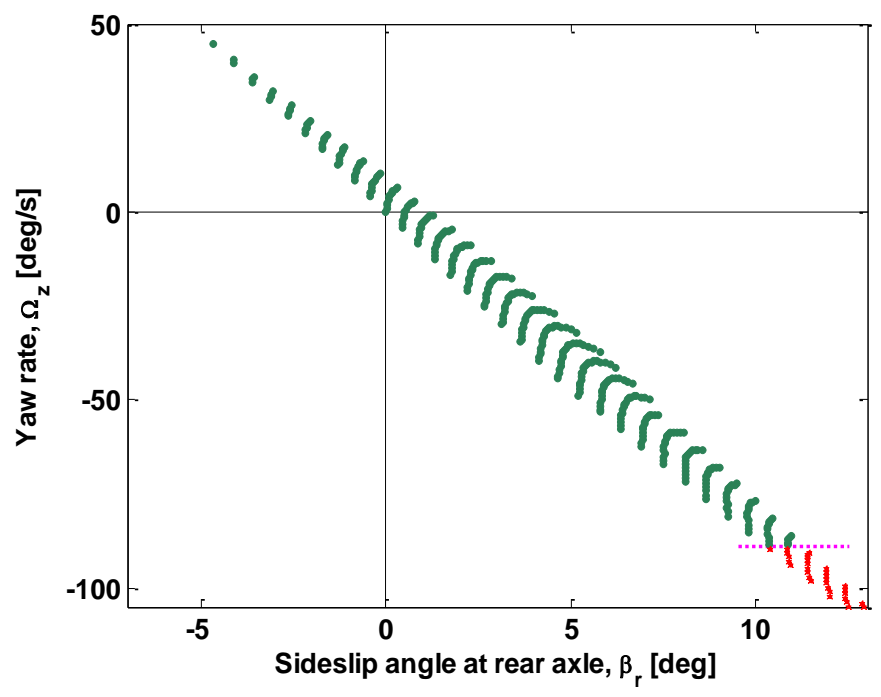

(b)

Figure 1.8 Effective range of ESC (a) and PISC (b) on the phase plane. Each dot is an initial point of vehicle states created by an impact, and the purple dotted lines indicate the same criteria to evaluate performance. (Criteria: vehicle states within 1 second after the collision are in the specific ranges; heading angle $|\psi| \leq 25^{\circ}$, roll angle $|\phi| \leq 10^{\circ}$, lateral displacement $\left|Y_{C G}\right| \leq 1.25 \times$ lane width) [5] 
Recently, this type of secondary collision braking strategy became available on production vehicles $[38,39]$. For example, Audi has started to equip the "Secondary Collision Brake Assist" system in 2012 model A3s as standard equipment. It is estimated, in EURO New Car Assessment Program (NCAP) analysis [39], that around 8 percent of fatalities and 4 percent of serious injuries could be avoided, if all cars were equipped with this system. Figure 1.9 shows three example vehicle safety functions from different companies. The control strategies of (a) and (b) in Figure 1.9 apply full or partial braking on all four wheels after an impact is detected. Since this function reduces the vehicle's longitudinal speed significantly, kinetic energy from the initial impact is reduced and the severity of secondary collisions can be moderated. The algorithm used in (c) of Figure 1.9 is more preemptive. The system estimates the impending rear-end collision using a RADAR sensor, and the braking control action is initiated during or even before the impact. Although the braking function is activated only at a stationary state, it can help to prevent secondary accident and reduce the risk of whiplash injuries [8].

When a high yaw rate is generated, these simple full braking strategies may not effectively reduce the risk of secondary collisions. Since the full braking does not directly control the vehicle motion, the attenuation of lateral speed and yaw rate is a by-product of reduced vehicle forward speed, rather than the direct consequence of stability control [5]. For this reason, a vehicle with full braking may introduce an undesirable lateral deviation and exposure to a side impact. To consider the course of vehicle motion due to the initial collision, a trajectory optimization scheme that takes both the lateral deviation and the time duration into account is proposed in $[40,41]$. The control objective of this work is based on an analysis [9] showing that risks of secondary events could be lower if lateral deviations are reduced.

In summary, the concepts discussed in the literature aim to stabilize yaw motion, reduce vehicle speed, or reduce lateral deviation from its original uncontrolled course, but the threat of a vulnerable heading angle to subsequent collisions with another moving vehicle or stationary object still exists [42]. Considering the fact that the sides of vehicles are more vulnerable than the front and rears in terms of absorbing crash energy and shielding occupants, accidents in side impact collisions result in more severe injury than 
accidents with front or rear-end collisions. Therefore, the vehicle heading angle is also an important vehicle state that needs to be considered for secondary collision safety.
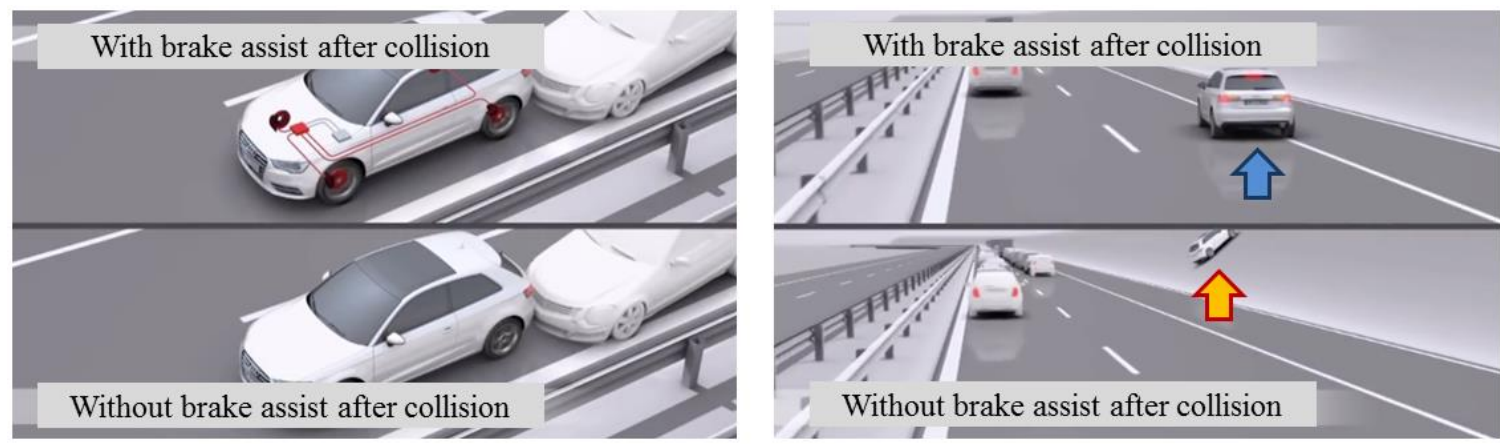

(a) Audi, Secondary Collision Brake Assist [43]
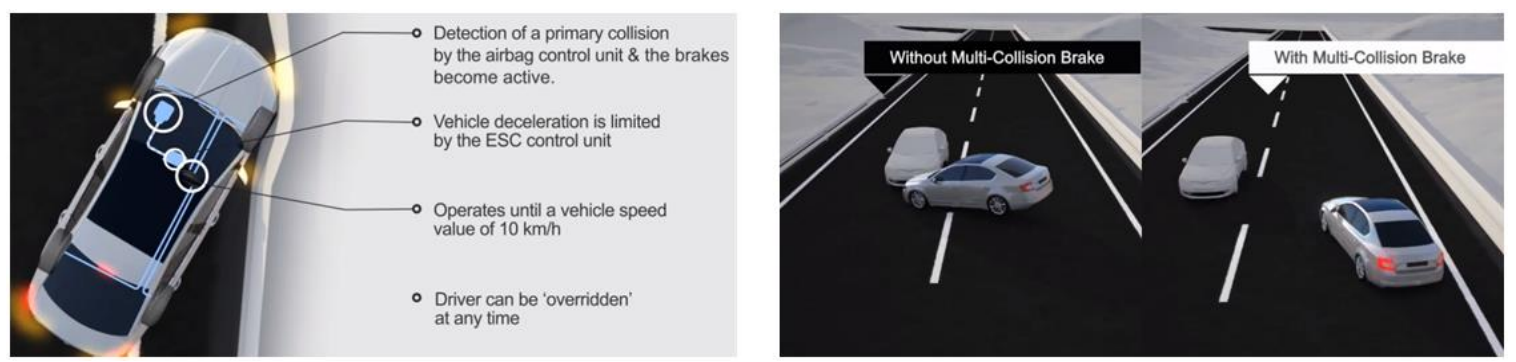

(b) Skoda, Multi-Collision Brake [44]

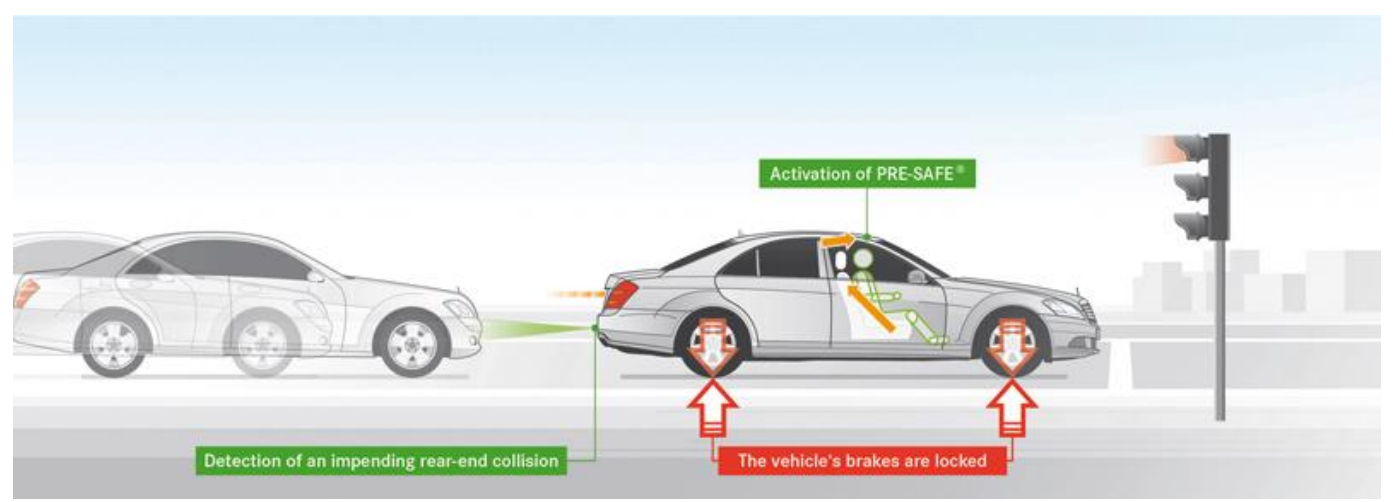

(c) Daimler, Pre-Safe Plus (Monitoring the traffic behind and applying brakes) [45]

Figure 1.9 Post impact safety applications available commercially 


\subsection{Objective and Contributions}

The objective of this dissertation is to develop an active safety system to control vehicle motion after an impact. There are two components in the research, the first includes the development of algorithms that estimate the strength of the collision force and predict the resulting vehicle motion. The collision estimation and motion prediction algorithm make it possible to initiate proper control actions as early as possible. The second part of the research is the development of the control algorithm. The main objective of the control system is to control vehicle motion so that the vehicle attains a safe heading angle while minimizing lateral displacement. Because the vehicle dynamics are nonlinear, the control should be designed considering the main nonlinear effects, namely the tire saturation. Thus, the constrained optimization framework is used. Using differential braking and active steering, the system can regulate heading angle, yaw rate, and lateral deviation to mitigate or avoid subsequent crashes. The process of achieving these objectives and the main contributions of this thesis are shown in the following:

\section{(1) Collision force estimation and vehicle motion prediction}

A methodology for collision force estimation and motion prediction is proposed. The estimation algorithm relies on a four degree of freedom vehicle model. A collision with another vehicle causes an abrupt change in vehicle motion. Within a short period of time, the vehicle can develop a substantial slip angle and yaw rate. Assuming that the on-board sensors can capture this abrupt change of vehicle state, the impulse strength can be estimated by using the vehicle dynamic model. The key feature of this algorithm is the prediction of the magnitude of the collision force in the early stage of a collision process, so that the resulting vehicle response can be predicted and the desired vehicle state can be determined promptly. In order to obtain reasonably accurate prediction, inter-sample changes in the estimated

impulse are monitored and the presumed pulse width is checked and corrected if necessary. Figure 1.10 illustrates the process of estimating impulses and predicting both the collision force and vehicle response. 


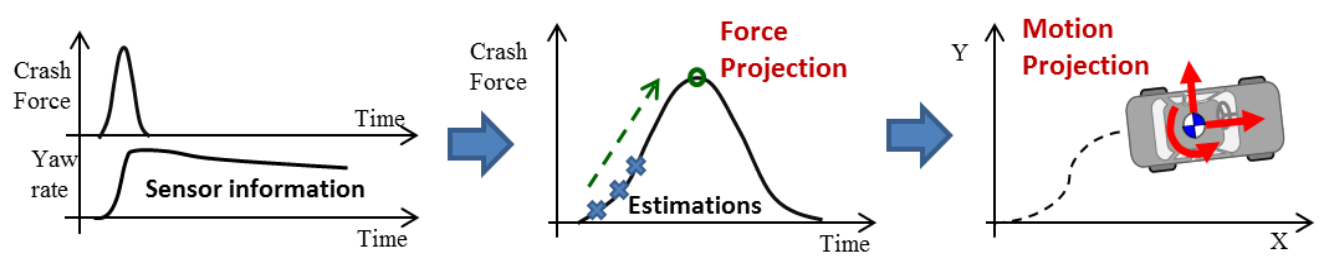

Figure 1.10 The overall procedure of estimating the collision force and predicting vehicle motion using the sensor information after the collision

(2) Development of an algorithm to determine the final vehicle orientation by analyzing vehicle dynamic characteristics

One of the key questions for controlling vehicle motion after an impact is how to effectively utilize the actuators, so that the risk of secondary collision is minimized. This thesis presents an analysis of stabilizing vehicle motion to multiple equilibria. The analysis is carried out by looking at the vehicle dynamic characteristics on the yaw rate - slip angle phase plane. Considering the fact that the control effectiveness reduces as the vehicle sideslip angle approaches \pm 90 degrees, the final desired vehicle state must be determined immediately after detecting a collision impact. When the impact is minor, it may make sense to bring the vehicle heading back to zero degrees. On the other hand, as the initial yaw rate increases, the final desired heading angles can be multiples of $180^{\circ}\left(180^{\circ}, 360^{\circ}, 540^{\circ}\right.$, etc. and their mirror states).

\section{(3) A hierarchical optimal controller for extreme vehicle maneuvers}

Optimal controllers are developed and integrated into a modular controller to provide a flexible design for vehicle motion control. Each module can be designed and tuned separately by exploiting designer's insight into the physical relationship between the inputs and outputs of individual modules. In this thesis, the controller is composed of two optimal control modules: Linear time-varying model predictive controller (upper-level controller) and optimal control allocator (lower-level controller). The upper level controller determines the desired control in the form of vehicle yaw moment and lateral forces to achieve the control objectives. To ensure that the control solutions are feasible, the optimal algorithm considers the nonlinear constraints due to tire nonlinearities. Because the tire forces are nonlinear at high 
slip angles, and the longitudinal and lateral tire forces are highly coupled, the full range of longitudinal and lateral tire force model is employed $\left(-180^{\circ} \leq\right.$ slip angle $\leq 180^{\circ}$ ), much beyond the range in typical vehicle control systems.

In the lower level controller, an optimal control allocator maps the desired vehicle level control demands onto individual commands at each wheel. Based on the tire slip angles, a constrained optimization problem is solved to obtain the wheel slip ratios. A series of simulations with varying collision severities are studied to confirm that both small lateral deviation and landing at a safe heading angle (in multiples of $180^{\circ}$ ) can be achieved.

\section{(4) Rule-based control design for real-time applications}

Optimal control techniques are computationally expensive, and many different measures have been devised to improve the computational speed of the MPC algorithm [46, 47]. However, even today it is still expensive to implement model predictive control in automotive embedded systems. The computation time for optimization is affected by the size of sampling time and the time horizon for optimization, and more importantly by the size of the system (number of states and inputs). On the other hand, a rule-based control is computationally inexpensive. In this thesis, four major control modes were found from the analysis of optimal control results, and a rule-based controller is developed.

\section{(5) Preemptive motion control strategy}

The vehicle control can start before the impact occurs. Since the major challenge in controlling excessive vehicle motions is preserving the control authority (avoiding tire saturation), vehicle steering action prior to the impact can provide more control effectiveness. The key idea of the proposed control strategy is to generate an opposite yaw moment against the expected impact as illustrated in Figure 1.11. The steering control is determined from the estimated collision force and vehicle motion prediction algorithm. An alternative version of this function using brake actuators is also studied. Similar to the skid-steering mechanism [48, 49], the vehicle can be steered by differential braking. The brake-steer is simple and readily available on all new passenger vehicles today. 


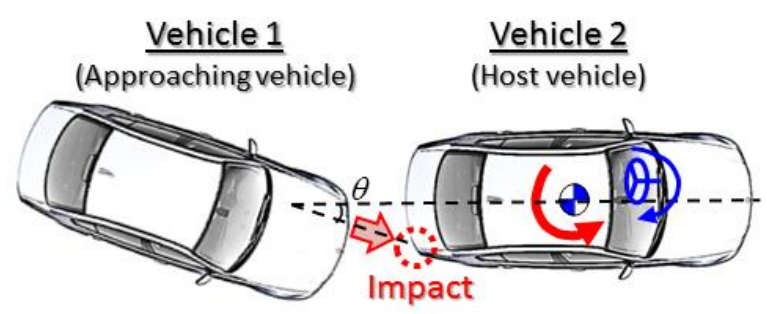

Figure 1.11 A crash scenario showing a preemptive steering control to negate the vehicle motion due to an impact

\subsection{Thesis Organization}

The remainder of this thesis is organized as follows. Chapter 2 presents the vehicle and tire models used in the thesis. Chapter 3 proposes a collision force estimation scheme and a vehicle motion prediction scheme. In Chapter 4, a procedure to determine the final desired vehicle state is presented. The design of a post-impact stability controller based on differential braking is presented in Chapter 5. The Linear Time-Varying Model Predictive Control (LTV-MPC) methodology is used to design the high-level controller. An optimal control allocation algorithm, which utilizes the relationship between the highlevel vehicle motion commands and independent braking forces, is presented. The effectiveness of the proposed control system, consisting of modules for impact detection and prediction, desired vehicle state generation, vehicle motion command, and braking force allocation, is demonstrated using computer simulations. Comparisons of the achieved control performance with other control strategies such as conventional ESC and full braking are also presented. In addition, the proposed rule-based control and preemptive steering control results are demonstrated. Finally, conclusions and future work are outlined in Chapter 6. 


\section{CHAPTER 2 SYSTEM MODELS}

In this chapter, the two system models for collision estimation and control design are discussed: a vehicle dynamic model and a tire model. In general, a vehicle modeled as a single rigid body has six degrees of freedom as shown in Figure 2.1. The axes of the local coordinate system are fixed at the vehicle CG. It consists of three translational and three rotational degrees of freedom. The translational components are defined along the vehicle's longitudinal, lateral, and vertical axes. The yaw is the rotational motion about the vertical axis, the roll is the one about the longitudinal axis, and the pitch is the one about the lateral axis.

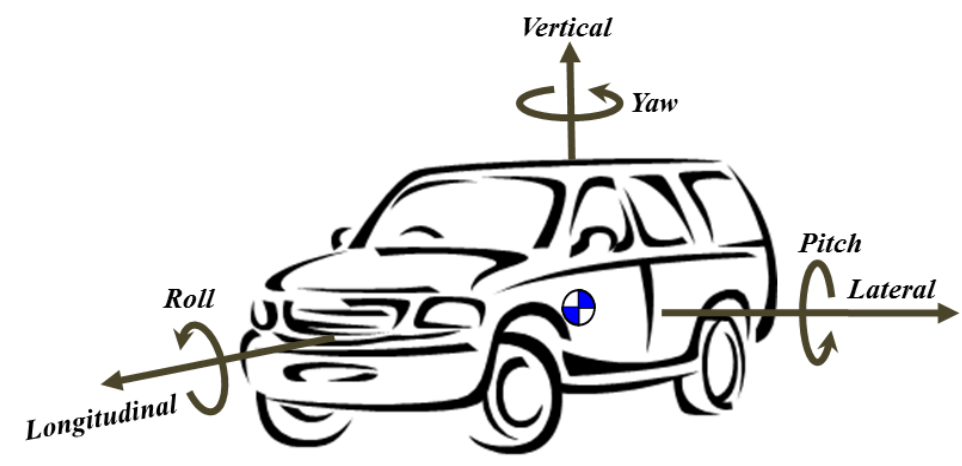

Figure 2.1 The body fixed vehicle coordinate system (ISO 8855)

Because of vehicle rotational motions and tire slip, the velocity of each wheel is not guaranteed to be aligned with the direction of the wheel. This means that the vehicle course angle can be different from the vehicle heading angle, and vehicle side slip exists. In Figure 2.2, the vehicle heading $(\psi)$, sideslip $(\beta)$, and course $(\gamma)$ angles are depicted. The vector $V$ points in the direction of the vehicle's velocity. The heading angle $(\psi)$ is the angle between the vehicle $\mathrm{x}$-axis and the $X$-axis of the earth-fixed coordinate system. Sideslip angle $(\beta)$ is the angle between the vehicle $x$-axis and the vehicle velocity vector at the vehicle CG. This angle indicates how much this vehicle is traveling sideways. The course angle $(\gamma)$ is the angle between the vehicle velocity vector at the vehicle CG and 
the horizontal axis ( $X$-axis) of the earth-fixed frame coordinate. Course angle is the sum of heading angle and sideslip angle $(\gamma=\psi+\beta)$.

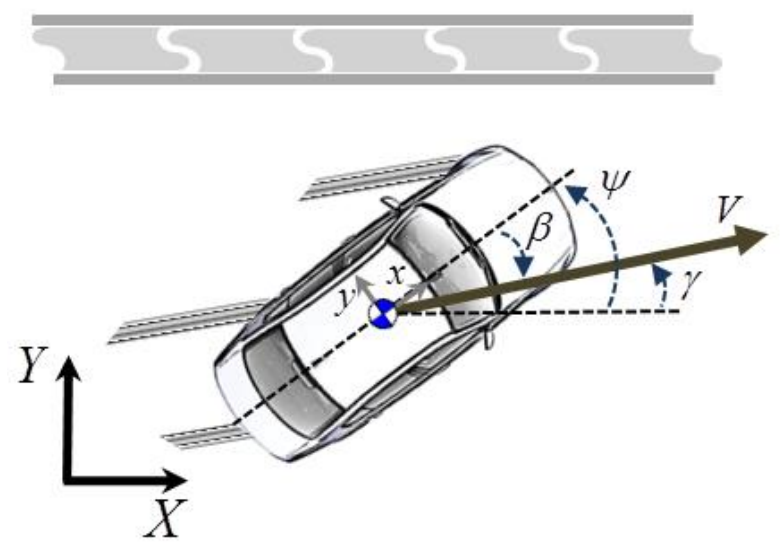

Figure 2.2 Vehicle motion shown with both the Earth-fixed frame $X$ - $Y$ and the vehiclefixed frame $x-y$

\subsection{Vehicle Model}

The main interest regarding the vehicle models in this research is on the change in vehicle kinematic states due to collisions. Therefore, changes in the $X-Y$ planar vehicle motions are the major concern. To make the problem manageable, the vehicle is treated as a rigid body with three degrees of freedom (DOF) (lateral, yaw, longitudinal). In [50], it was pointed out that the vehicle roll motion cannot be ignored in predicting the vehicle lateral and yaw motions after a collision.

Two vehicle models are used in this research. The first one is a 4-DOF model which includes vehicle longitudinal, lateral, yaw, and roll motions for better prediction of the vehicle motion after an impact. The other model is a 3-DOF (longitudinal, lateral, and yaw motion) model for vehicle motion control design. Since the vehicle position and heading controls are the focus of vehicle control, the vehicle roll motion is not considered in the control design model. It should be noted that rollover is also a type of secondary events which could be devastating; the roll mitigation function may need to be addressed explicitly for certain vehicle type but this will not be considered in this dissertation. 


\subsubsection{Four Degrees of Freedom Model}

It was shown in [5] that the 4-DOF vehicle model is a proper model to use in estimating the impulse and characterizing the vehicle sideslip, yaw, and roll motions induced by the impact. The vehicle model in this section will be used to estimate the impulse and predict the vehicle motion. The schematic diagram of the vehicle model is shown in Figure 2.3. This vehicle model separates the rolling (sprung) mass $m_{R}$ from the non-rolling (unsprung) mass $m_{N R}$. The suspension elements at the four corners are lumped into an equivalent torsional spring and a damper around the roll axis. The overall mass of the vehicle is denoted as $m$. The coordinate system $x-y-z$ is fixed on the vehicle body, and the orientation conforms to the ISO coordinate convention. The roll axis (the same as the $\mathrm{x}$-axis here) passes through the non-rolling mass and is assumed to be parallel to the ground. $a, b$, and $T_{W}$ are the distances from the front and rear axles to the $\mathrm{CG}$ and the track width of the vehicle. The distance between the rolling mass CG and the roll axis is denoted as $h$, whereas the height of the overall CG above the ground is denoted as $h_{C G}$.

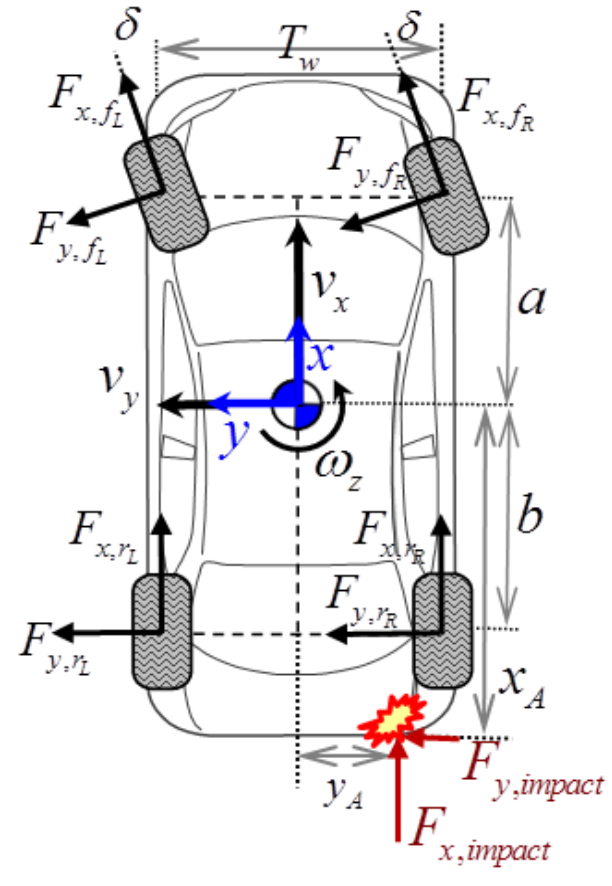

(a) Top view

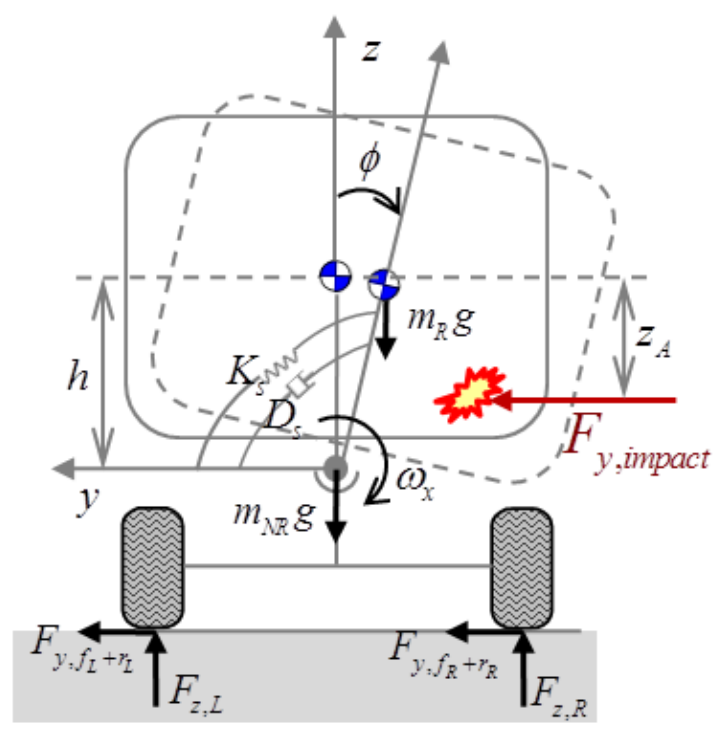

(b) Rear view

Figure 2.3 Schematic diagrams of the 4-DOF vehicle model with impact forces applied 
Figure 2.3 also shows the vehicle longitudinal velocity $\left(v_{x}\right)$, lateral velocity $\left(v_{y}\right)$, yaw rate $\left(\omega_{z}\right)$, roll angle $(\phi)$, and roll rate $\left(\omega_{x}\right)$. The impact forces $\left(F_{x, \text { impact }}, F_{y, \text { impact }}\right)$ are assumed to be acting along the horizontal plane. The impact position (A) is at $\left(x_{A}, y_{A}, z_{A}\right)$. The dynamic equations can be written as

\section{$\underline{\text { Longitudinal motion }}$}

$$
m\left(\dot{v}_{x}-v_{y} \omega_{z}\right)=F_{x, \text { impact }}+\left(F_{x, f_{L}}+F_{x, f_{R}}\right) \cos \delta-\left(F_{\mathrm{y}, f_{L}}+F_{\mathrm{y}, f_{R}}\right) \sin \delta+\left(F_{x, r_{L}}+F_{x, r_{R}}\right)
$$

\section{$\underline{\text { Lateral motion }}$}

$$
\begin{aligned}
m\left(\dot{v}_{y}+v_{x} \omega_{z}\right)-m_{R} h \dot{\omega}_{x} & =F_{y, \text { impact }}+\left(F_{y, r_{L}}+F_{y, r_{R}}\right)+\left(F_{x, f_{L}}+F_{x, f_{R}}\right) \sin \delta \\
& +\left(F_{\mathrm{y}, f_{L}}+F_{\mathrm{y}, f_{R}}\right) \cos \delta
\end{aligned}
$$

Yaw motion

$$
\begin{aligned}
& I_{z z} \dot{\omega}_{z}+I_{x z} \dot{\omega}_{x}=x_{A} F_{y, \text { impact }}-y_{A} F_{x, i m p a c t}+a\left(F_{x, f_{L}}+F_{x, f_{R}}\right) \sin \delta+a\left(F_{y, f_{L}}+F_{y, f_{R}}\right) \cos \delta \\
& -b\left(F_{y, r_{L}}+F_{y, r_{R}}\right)+T_{W} / 2\left(F_{x R R}-F_{x R L}\right)+T_{W} / 2\left[\left(F_{x, f_{R}}-F_{x, f_{L}}\right) \cos \delta-\left(F_{y, f_{R}}-F_{y, f_{L}}\right) \sin \delta\right]
\end{aligned}
$$

\section{$\underline{\text { Roll motion }}$}

$$
\begin{gathered}
I_{x x, s} \dot{\omega}_{x}+I_{x z} \dot{\omega}_{z}-m_{R} h\left(\dot{v}_{y}+v_{x} \omega_{z}\right)=F_{y, \text { impact }}\left(z_{A}-h\right)+\left(m_{R} g h-K_{s}\right) \phi-D_{s} \omega_{x} \\
\text { where, } \quad \begin{array}{l}
F_{y, f_{L}}=C_{f}\left(\alpha_{f_{L}}\right) \cdot \alpha_{f_{L}}, \quad F_{y, f_{R}}=C_{f}\left(\alpha_{f_{R}}\right) \cdot \alpha_{f_{R}} \\
F_{y, r_{L}}=C_{r}\left(\alpha_{r_{L}}\right) \cdot \alpha_{r_{L}}, \quad F_{y, r_{R}}=C_{r}\left(\alpha_{r_{R}}\right) \cdot \alpha_{r_{R}}
\end{array}
\end{gathered}
$$

The vehicle yaw moment of inertia about $z$-axis is denoted as $I_{z z} . I_{x x s}$ is the roll moment of inertia of the sprung mass with respect to the $x$-axis, $I_{x z}$ is the product of inertia of the sprung mass about the $x$-axis and $z$-axis, and $K_{s}, D_{s}$ are roll stiffness and damping coefficients of the lumped suspension. It should be noted that the lateral tire forces $\left(F_{y, f_{L}}, F_{y, f_{R}}, F_{y, r_{L}}, F_{y, r_{R}}\right)$ vary nonlinearly with the tire slip angles. Instead of using constant cornering stiffness $\left(C_{f}, C_{r}\right)$, more elaborate tire force model is used which will be described in Section 2.2.2.

\subsubsection{Three Degrees of Freedom Model}

The objective of the control system is to manage the vehicle position and orientation with respect to the road. In order to consider trajectories with extreme vehicle motions, such as spinout, it is appropriate to define the vehicle model in the earth-fixed 
frame $(X-Y$ axes). For this reason, the local longitudinal and lateral components with respect to the body-fixed coordinate are transformed to those in the earth-fixed frame.

$$
\left[\begin{array}{l}
X \\
Y
\end{array}\right]=\left[\begin{array}{cc}
\cos (\psi) & -\sin (\psi) \\
\sin (\psi) & \cos (\psi)
\end{array}\right]\left[\begin{array}{l}
x \\
y
\end{array}\right]
$$

And, tire forces are also presented in the earth-fixed frame coordinates.

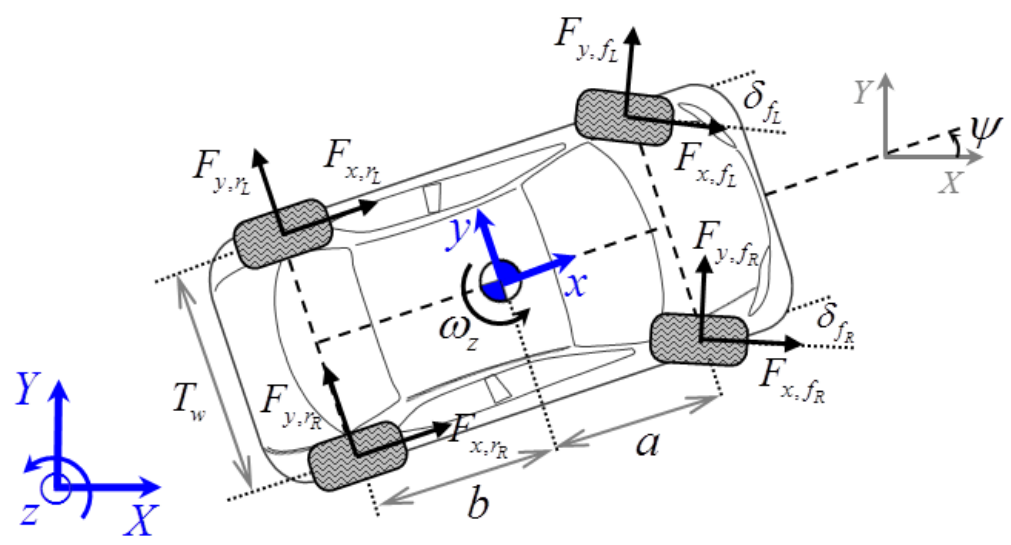

Figure 2.4 Schematic diagram of the 3-DOF vehicle model

The planar nonlinear dynamics can be represented by the following equations:

$$
\begin{aligned}
& \dot{X}=V_{X} \\
& m \cdot \dot{V}_{X}=F_{x, f_{L+R}} \cdot \cos (\psi+\delta)+F_{x, r_{L+R}} \cdot \cos (\psi)-F_{y, f_{L+R}} \cdot \sin (\psi+\delta)-F_{y, r_{L+R}} \cdot \sin (\psi) \\
& \dot{Y}=V_{Y} \\
& \begin{array}{l}
m \cdot \dot{V}_{Y}=F_{x, f_{L+R}} \cdot \sin (\psi+\delta)+F_{x, r_{L+R}} \cdot \sin (\psi)+F_{y, f_{L+R}} \cdot \cos (\psi+\delta)+F_{y, r_{L+R}} \cdot \cos (\psi) \\
\dot{\psi}=\omega_{z} \\
I_{z z} \cdot \dot{\omega}_{z}=a \cdot\left(F_{x, f_{L+R}} \cdot \sin (\delta)+F_{y, f_{L+R}} \cdot \cos (\delta)\right)-b \cdot F_{y, r_{L+R}} \\
\quad+\frac{T_{W}}{2} \cdot\left\{\left(F_{x, f_{R}}-F_{x, f_{L}}\right) \cdot \cos (\delta)+\left(F_{x, r_{R}}-F_{x, r_{L}}\right)-\left(F_{y, f_{R}}-F_{y, f_{L}}\right) \cdot \sin (\delta)\right\}
\end{array}
\end{aligned}
$$


The longitudinal and lateral forces for each tire are denoted by $F_{x, \oplus_{\ominus}}, F_{y, \oplus_{\ominus}}$ which are defined in a tire-fixed coordinate. The subscript $(\bigoplus \in[f, r])$ represents the front and rear axles and the next subscript denotes the left and right sides of the vehicle $(\ominus \in[L, R, L+R]) . x$ and $y$ are the axes located at the $C G$ in the vehicle fixed frame, while $X$ and $Y$ indicate vehicle position in the earth-fixed frame. The motion of the vehicle is defined by vehicle velocities $V_{X}, V_{Y}$ associated with $X$ and $Y$, the heading angle $\psi$, and the yaw rate $\omega_{z} \cdot a, b$, and $T_{W}$ are the distances from the front and rear axles to the $\mathrm{CG}$ and the track width of the vehicle. $m$ is the vehicle mass and $I_{z z}$ is the vehicle yaw moment of inertia.

\subsection{Tire Model}

Tire force generation is the main source of nonlinearity in vehicle dynamics. Tires are reasonably linear at small tire slip angles and on high frictional roads. However, if the tire operates near its force generation limit, nonlinear tire models must be used. Figure 2.5 shows an example set of experimental tire force data illustrating the nonlinear and coupling effects. Each line represents a set of combined tire forces under constant slip angle. It shows that the lateral force is reduced as tire traction / braking force is increased. Also, the maximum brake force that a tire can generate is reduced as slip angle increases.

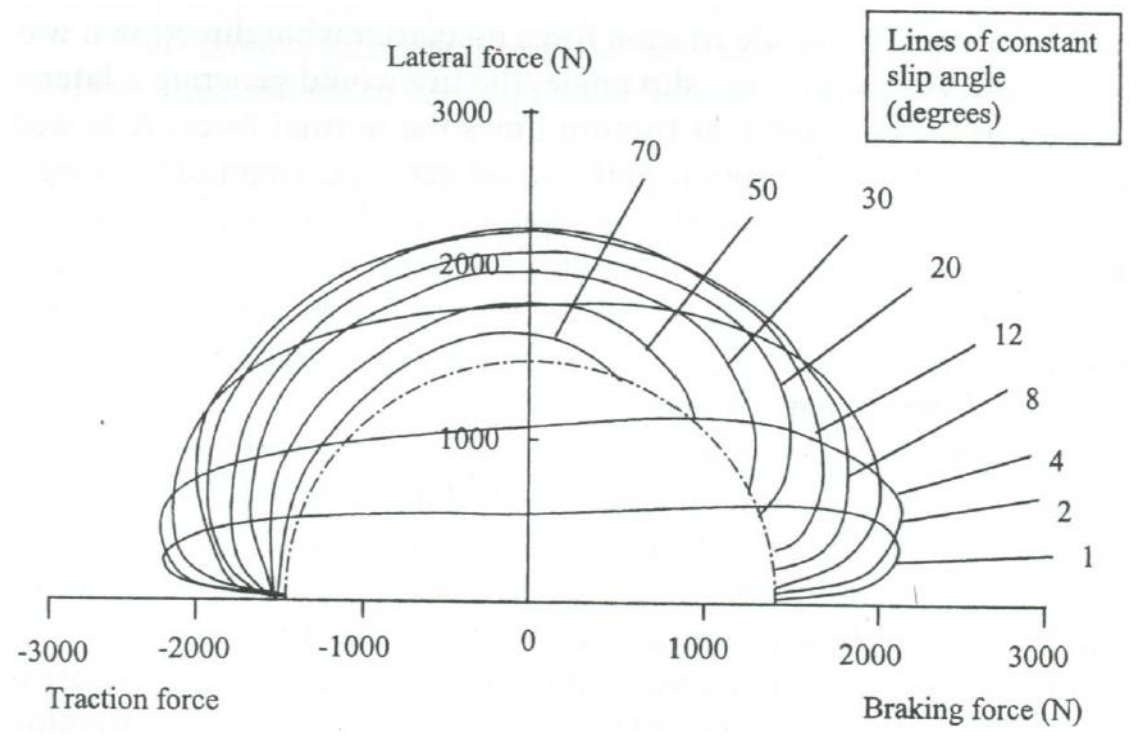

Figure 2.5 Experimental data showing combined lateral and longitudinal tire forces [51] 
The locked wheel case is indicated by the dash-dot line which shows that the amplitudes of the total tire forces are almost constant [51]. In this research, the nonlinear tire force is represented by the combined slip Pacejka's model [52], which is one of the most commonly used empirical models. This model uses empirically calibrated functions to describe the tire force profile based on a given slip ratio, slip angle, tire vertical load and surface friction coefficient [53].

\subsubsection{Pacejka Tire Model}

The Pacejka tire model, also known as the Magic Formula (MF) model, is capable of matching experimental data well by adjusting parameters of the formula.

The basic form of the Pacejka tire model is described in the following equations:

$$
y=D \sin \{C \arctan [B x-E(B x-\arctan B x)]\}
$$

where the output variable y can be the tire longitudinal force, lateral force, or aligning moment with the input variable $\mathrm{x}$ being slip angle or slip ratio. The model parameters are

functions of the tire normal load, camber angle, and road friction coefficient. They are termed stiffness factor (B), shape factor (C), peak value (D), and curvature factor (E) respectively. To handle the longitudinal and lateral force coupling effect on the tire, we use the combined-slip formula as shown in $[5,52]$.

$$
\left[\begin{array}{ll}
F_{x, \text { tire }} & F_{y, \text { tire }}
\end{array}\right]^{T}=\Phi\left(\frac{C_{\alpha}\|s\|}{F_{p}\left(F_{z}\right)}, C, E\right) \cdot \frac{F_{p}\left(F_{z}\right)}{\|s\|} \cdot s
$$

where,

$$
\begin{gathered}
\Phi(\chi, C, E)=\sin \left[C \cdot \operatorname{acrtan}\left\{\frac{\chi}{C}-E\left(\frac{\chi}{C}-\arctan \left(\frac{\chi}{C}\right)\right)\right\}\right] \\
s=\left[\begin{array}{ll}
s_{x} & s_{y}
\end{array}\right]^{T}=[\lambda \tan \alpha]^{T} \\
F_{p}\left(F_{z}\right)=F_{z} /\left(1+\left(1.5 \cdot \frac{F_{z}}{m \cdot g}\right)^{3}\right) \\
C_{\alpha}=c_{1} \cdot m \cdot g \cdot\left(1-e^{-\frac{c_{2} \cdot F_{z}}{m \cdot g}}\right)
\end{gathered}
$$


Here, $m$ is the nominal vehicle mass that effectively determines the tire loading condition. And the cornering stiffness $C_{\alpha}$ changes with the tire normal load and saturates when the normal load is sufficiently high. The parameters $c_{1}$ and $c_{2}$ are dimensionless constants obtained through data-fitting. In this study, road friction coefficient $(\mu)$ is assumed to be fixed on dry asphalt $(\mu=0.7)$, which determines parameters for the tire model. The tire slip angles are defined as the angle between the velocity vector of the wheel and the geometric heading of the wheel, as illustrated in Figure 2.6. When slip angle is generated by the lateral deformation of the tire contact region, it gives rise to lateral tire force.

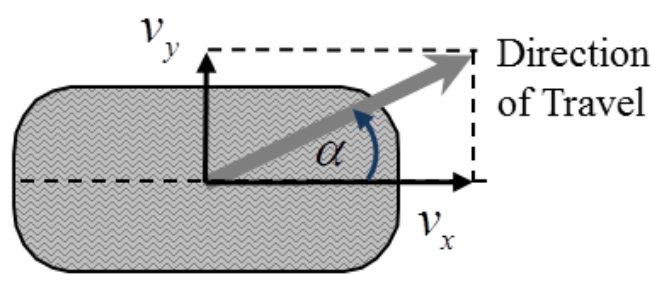

Figure 2.6 Tire slip angle between a wheel centerline and the direction of travel

Slip angle on each tire is computed from:

$$
\begin{gathered}
\alpha_{f_{L}}=-\delta+\arctan \frac{v_{y}+a \cdot \omega_{z}}{v_{x}-\left(T_{W} / 2\right) \cdot \omega_{z}} \\
\alpha_{f_{R}}=-\delta+\arctan \frac{v_{y}+a \cdot \omega_{z}}{v_{x}+\left(T_{W} / 2\right) \cdot \omega_{z}} \\
\alpha_{f_{L}}=\arctan \frac{v_{y}-b \cdot \omega_{z}}{v_{x}-\left(T_{W} / 2\right) \cdot \omega_{z}} \\
\alpha_{f_{R}}=\arctan \frac{v_{y}-b \cdot \omega_{z}}{v_{x}+\left(T_{W} / 2\right) \cdot \omega_{z}}
\end{gathered}
$$

Wheel normal loads are computed by:

$$
F_{z, f_{L}}=\frac{m}{2} \cdot\left(\frac{b}{L} \cdot g-\frac{h_{C G}}{L} \cdot A_{x}\right)-\frac{K_{s, f}}{K_{s, f}+K_{s, r}} \cdot\left(\frac{h_{C G}}{T_{W}} \cdot m \cdot A_{y}+\frac{h}{T_{W}} \cdot m_{R} \cdot g \cdot \sin \phi\right)
$$




$$
\begin{aligned}
& F_{z, f_{R}}=\frac{m}{2} \cdot\left(\frac{b}{L} \cdot g-\frac{h_{C G}}{L} \cdot A_{x}\right)+\frac{K_{s, f}}{K_{s, f}+K_{s, r}} \cdot\left(\frac{h_{C G}}{T_{W}} \cdot m \cdot A_{y}+\frac{h}{T_{W}} \cdot m_{R} \cdot g \cdot \sin \phi\right) \\
& F_{z, r_{L}}=\frac{m}{2} \cdot\left(\frac{a}{L} \cdot g+\frac{h_{C G}}{L} \cdot A_{x}\right)-\frac{K_{s, r}}{K_{s, f}+K_{s, r}} \cdot\left(\frac{h_{C G}}{T_{W}} \cdot m \cdot A_{y}+\frac{h}{T_{W}} \cdot m_{R} \cdot g \cdot \sin \phi\right) \\
& F_{z, r_{R}}=\frac{m}{2} \cdot\left(\frac{a}{L} \cdot g+\frac{h_{C G}}{L} \cdot A_{x}\right)+\frac{K_{s, r}}{K_{s, f}+K_{s, r}} \cdot\left(\frac{h_{C G}}{T_{W}} \cdot m \cdot A_{y}+\frac{h}{T_{W}} \cdot m_{R} \cdot g \cdot \sin \phi\right)
\end{aligned}
$$

where, $K_{s, f}$ and $K_{s, r}$ are the roll stiffness of front and rear suspensions.

Figure 2.7 shows the longitudinal and lateral tire force profiles over the entire range of the tire slip angle $\left(-180^{\circ} \leq \alpha \leq 180^{\circ}\right)$ with varying longitudinal slip ratio $(\lambda)$. When $\alpha$ is near 0 or $\pm 180^{\circ}$, both longitudinal and lateral forces vary significantly with $\lambda$. On the contrary, the effects of $\lambda$ are small when $\alpha$ is around $\pm 90^{\circ}$, meaning that braking action is not effective in manipulating tire forces.
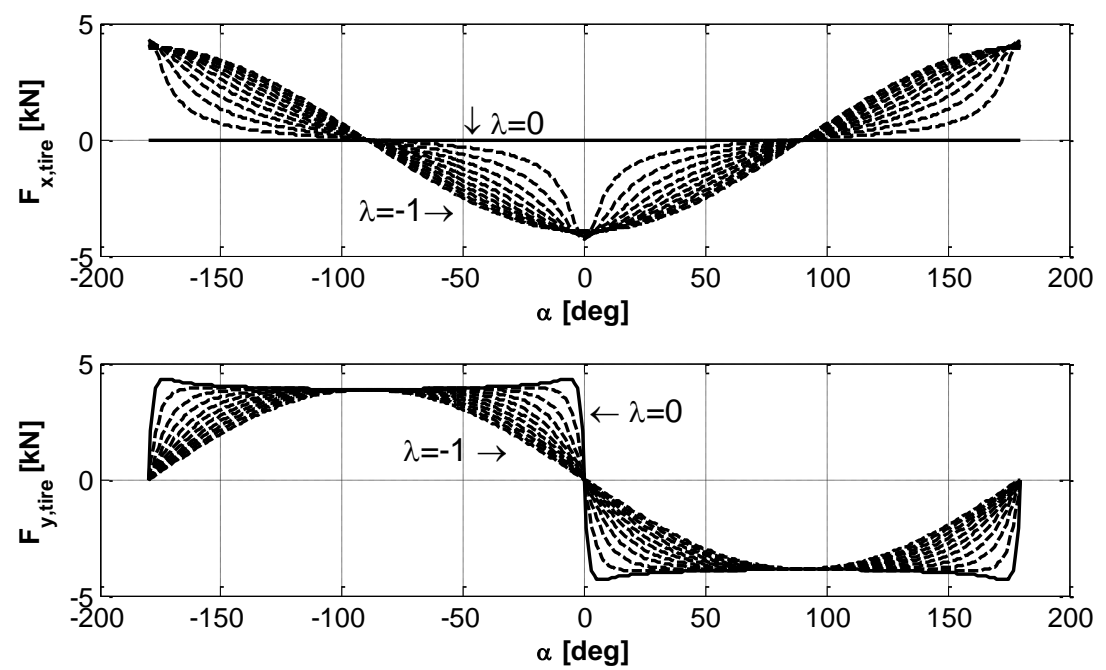

Figure 2.7 Longitudinal and lateral tire forces as functions of the tire slip angle and longitudinal slip ratio $(-1 \leq \lambda \leq 0)$

To understand the coupling effects of tire longitudinal and lateral forces, the concept of "Friction circle" is used to graphically represent tire traction capability in braking and steering condition. To review the result of tire model and analyze coupling 
effect inside the friction circle, experimental data on $F_{x}-F_{y}$ plane [51] are compared to results in Figure 2.7 showing forces on the $\alpha-F_{x}$ and $\alpha-F_{y}$ planes. As shown in Figure 2.8, each curve on the $F_{x}-F_{y}$ plane is approximately a curve, but the size and shape of the circles vary with the slip angle. Two slip angle values are highlighted in blue and red lines for comparison. The tire with $8^{\circ}$ slip angle has large operating ranges in both longitudinal and lateral forces, while the tire in high slip angle $\left(70^{\circ}\right)$ has narrower ranges.
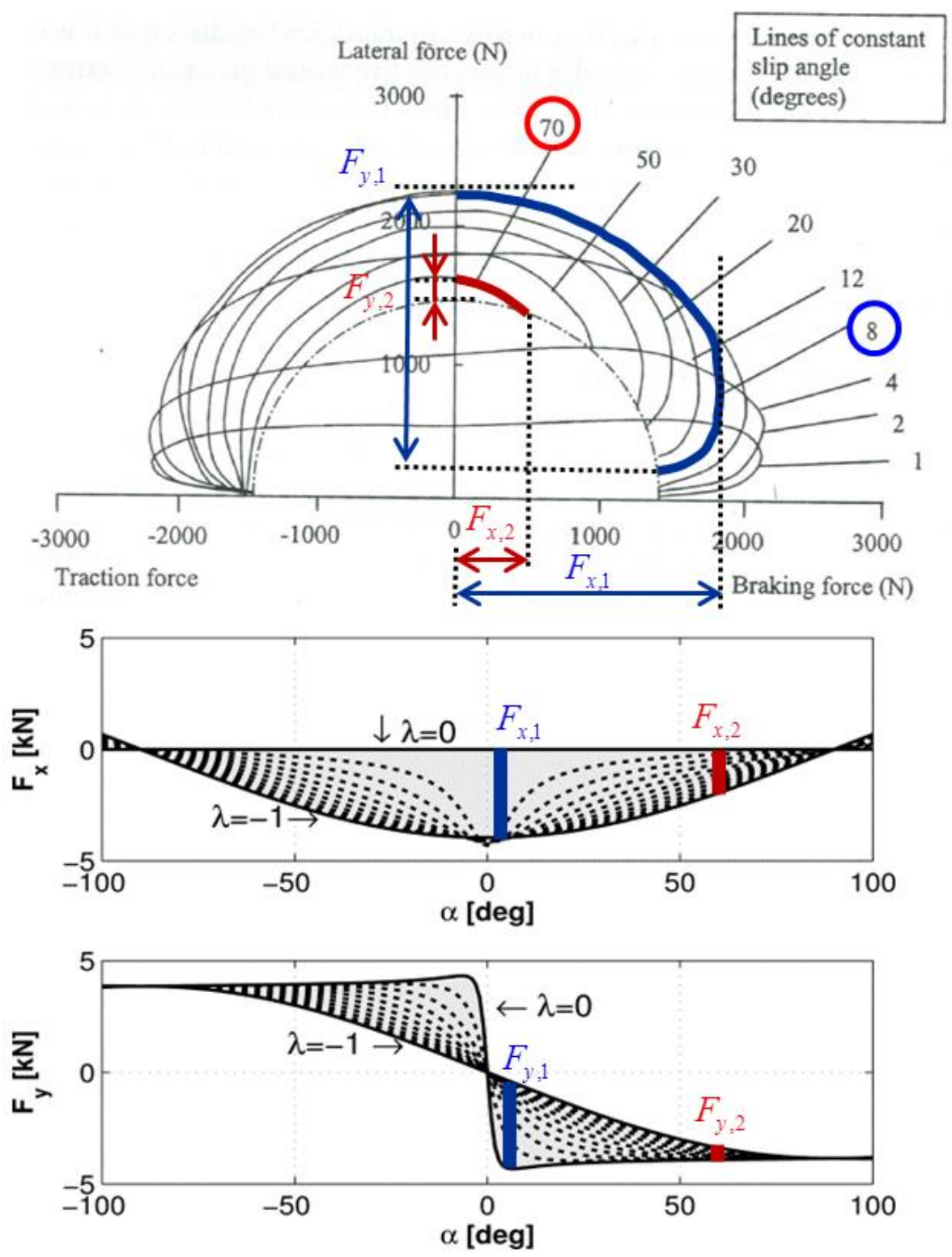

Figure 2.8 Plots to show the coupling nature of tire force generations 


\subsubsection{Effective Cornering Stiffness}

One of the most popular lateral tire models used in vehicle dynamics is a linear model:

$$
F_{y}=-C_{\alpha} \cdot \alpha
$$

When the slip angle is small, the tire cornering stiffness $\left(C_{\alpha}\right)$ is nearly constant. When the tire lateral force exceeds half of the maximum value (at slip angles larger than about 1 degree in Figure 2.9), the linear model is no longer accurate.

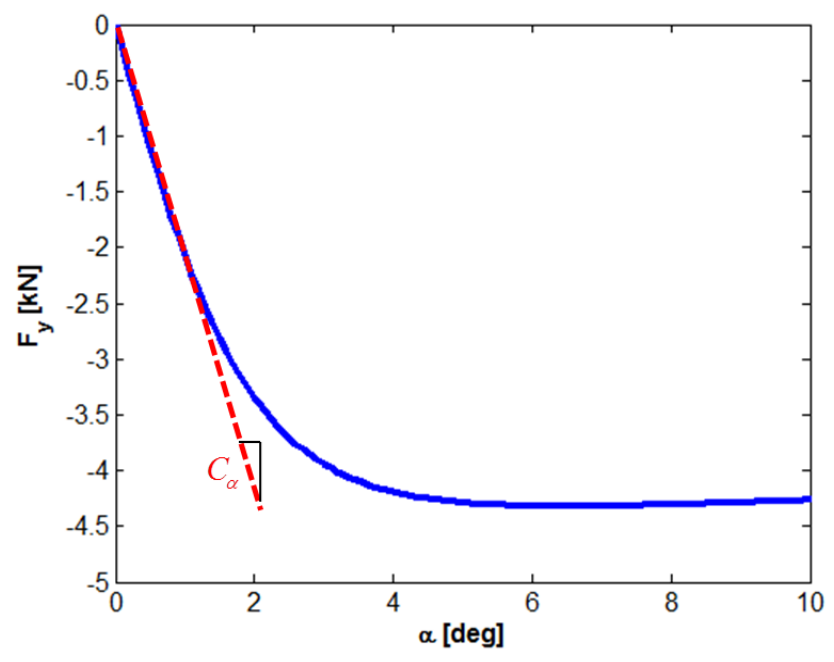

Figure 2.9 Tire Lateral Force and Slip Angle

In this case, as shown in $[54,55]$, an effective factor $(\eta)$ can be used to adjust the slope of the cornering stiffness to capture the nonlinear force profile. The value $\eta$ can vary between zero and one. When the slip angle $\alpha$ is close to 90 degrees, $\eta$ approaches zero. This effective factor is defined by the following equation:

$$
\eta(\alpha, \lambda)=-\frac{F_{y}(\alpha, \lambda)}{C_{\alpha} \cdot \alpha}
$$

Once $\eta$ is calculated based on $\alpha$ and $\lambda$, all the nonlinearity of the tire lateral force can be captured using a modified cornering stiffness $\left(\eta \cdot C_{\alpha}\right)$. Although Figure 2.10 only shows free rolling $(\lambda=0)$ and fully locked $(\lambda=-1)$ cases, more lines of $\eta$ can be drawn $(-1<\lambda<0)$. For more detailed analysis, this effective factor can also vary 
depending on the loading condition $\left(F_{z}\right)$. In this case, the dependent variables for $\eta$ are $\alpha, \lambda$, and $F_{z}$.

In Chapter 5, this formulation plays an important role in linearizing the nonlinear vehicle model at each operational point and to make it easy to formulate the controller structure without dealing with the mathematical formulation of the Magic Formula tire model.

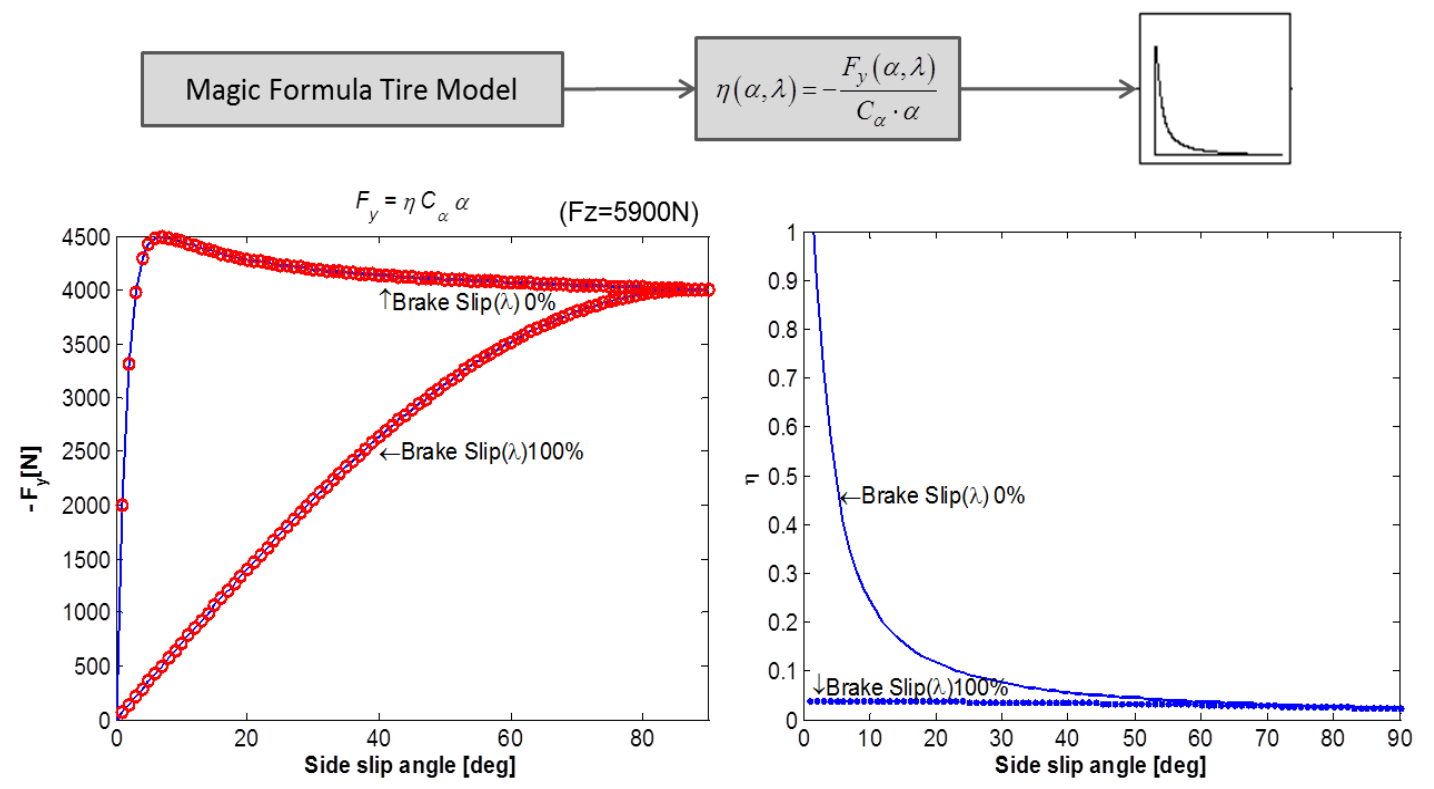

Figure 2.10 Procedure to obtain the correction factors for the effective cornering stiffness

\subsection{Parameters for Simulations}

To verify the fidelity of the 4-DOF model, comparison with simulation results from CarSim, a commercial nonlinear multi-body vehicle model, is examined. CarSim is widely used and the software has been validated against experimental test data [56, 57]. 4-DOF model parameters are summarized in Table 2.1. Responses to the same disturbance input are compared, and the parameters are tuned to achieve close agreement with CarSim. It is assumed that the vehicle travels straight with a speed of $30 \mathrm{~m} / \mathrm{s}(67$ $\mathrm{mph}, 108 \mathrm{kph}$ ) with no initial lateral speed, yaw rate, and roll rate before the impact. The impact is assumed to be located at $0.1 \mathrm{~m}$ to the right on the rear bumper and $0.66 \mathrm{~m}$ above the ground. Three simulation results in Figure 2.11, Figure 2.12, and Figure 2.13 show 
the responses from both models after external impact disturbances. The impact conditions induce different levels of accelerations: $3.7 \mathrm{~g}\left(A_{x}=3.4 g, A_{y}=1.5 g\right)$ in Figure 2.11, $0.97 \mathrm{~g}\left(A_{x}=0.67 g, A_{y}=0.7 g\right)$ in Figure 2.12, and $2.2 \mathrm{~g}\left(A_{x}=1.7 g, A_{y}=1.4 g\right)$ in Figure 2.13. In the figures, solid lines represent results from the CarSim model and dashed lines are from the 4-DOF vehicle model. The results indicate that the 4-DOF model is reasonably close to the CarSim results and it will be used for prediction/control later in this dissertation. Because CarSim includes each detail suspension dynamics at the four corners, discrepancies in yaw rate and roll rate motions increase over time. More detailed analysis about the vehicle motion characteristics after an impact will be presented in Section 4.2.

Table 2.1 Vehicle parameters for the 4-DOF model

\begin{tabular}{|c|c|c|c|}
\hline Parameters & Description & Value & Unit \\
\hline$M$ & Total vehicle mass & 2450 & $\mathrm{~kg}$ \\
\hline$m_{R}$ & Rolling mass & 2210 & $\mathrm{~kg}$ \\
\hline$m_{N R}$ & Non-rolling mass & 240 & $\mathrm{~kg}$ \\
\hline$a, b$ & Distance from axles to vehicle CG & $\begin{array}{r}1.105, \\
1.745\end{array}$ & $\mathrm{~m}$ \\
\hline$L$ & Wheelbase & 2.850 & $\mathrm{~m}$ \\
\hline$T_{W}$ & Track width & 1.600 & $\mathrm{~m}$ \\
\hline$h_{C G}$ & CG height above the ground & 0.66 & $\mathrm{~m}$ \\
\hline$h$ & Distance from sprung mass $\mathrm{CG}$ to the roll axis & 0.40 & $\mathrm{~m}$ \\
\hline$I_{z z}$ & Vehicle yaw moment of inertia about $z$ axis & 4946 & $\mathrm{~kg} \cdot \mathrm{m}^{2}$ \\
\hline$I_{x z}$ & Sprung mass product of inertia about roll and yaw axes & 40 & $\mathrm{~kg} \cdot \mathrm{m}^{2}$ \\
\hline$I_{x x s}$ & Sprung mass roll moment of inertia about the roll axis & 1597 & $\mathrm{~kg} \cdot \mathrm{m}^{2}$ \\
\hline$K_{s}$ & Total suspension roll stiffness & 94000 & $\mathrm{~N} \cdot \mathrm{m} / \mathrm{rad}$ \\
\hline$D_{s}$ & Total suspension roll damping & 8000 & $\mathrm{~N} \cdot \mathrm{m} \cdot \mathrm{s} / \mathrm{rad}$ \\
\hline$C_{f}$ & Front axle cornering stiffness & 145750 & $\mathrm{~N} / \mathrm{rad}$ \\
\hline$C_{r}$ & Rear axle cornering stiffness & 104830 & $\mathrm{~N} / \mathrm{rad}$ \\
\hline
\end{tabular}




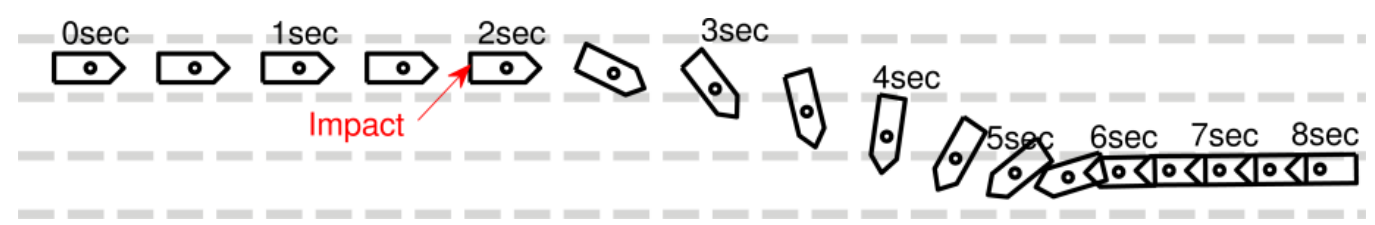

(a) Vehicle trajectories
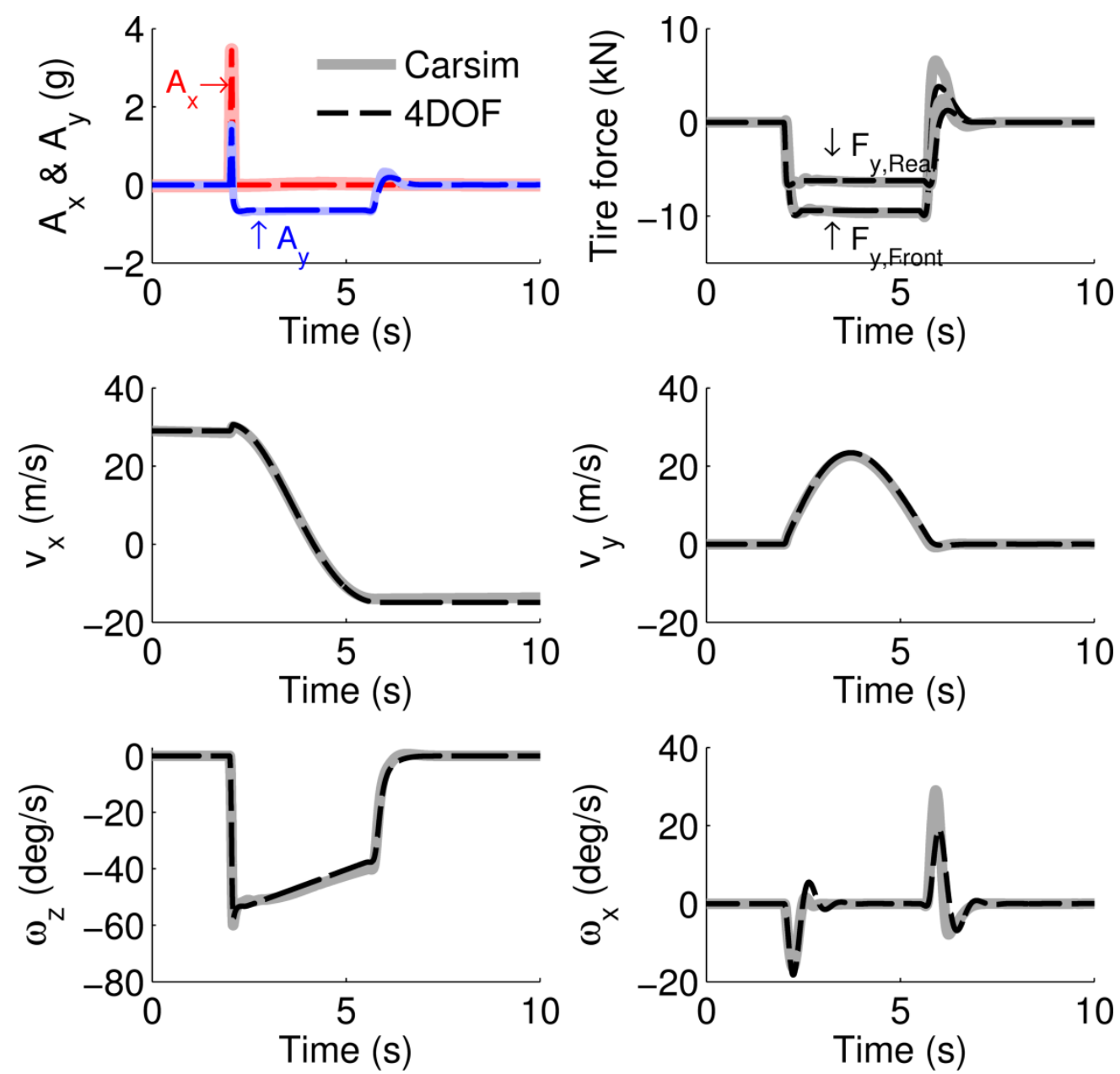

(b) Vehicle maneuver comparison

Figure 2.11 Vehicle response after an impact (No control action is involved. Initial conditions: $v_{x}=30 \mathrm{~m} / \mathrm{s}$, the impact generates $A_{x}=3.4 \mathrm{~g}, A_{y}=1.5 \mathrm{~g}$ ) 


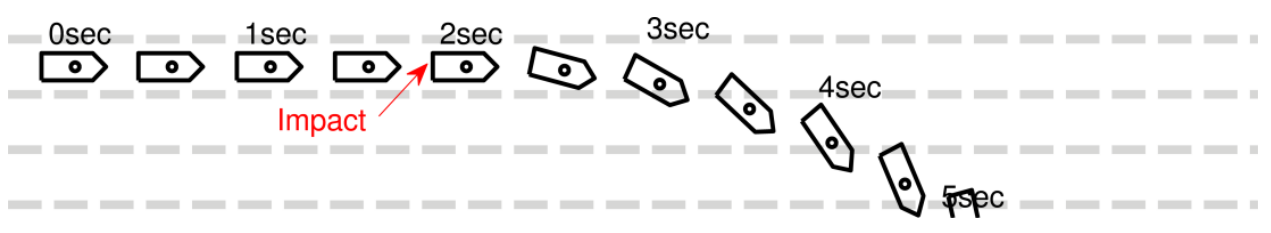

(a) Vehicle trajectories
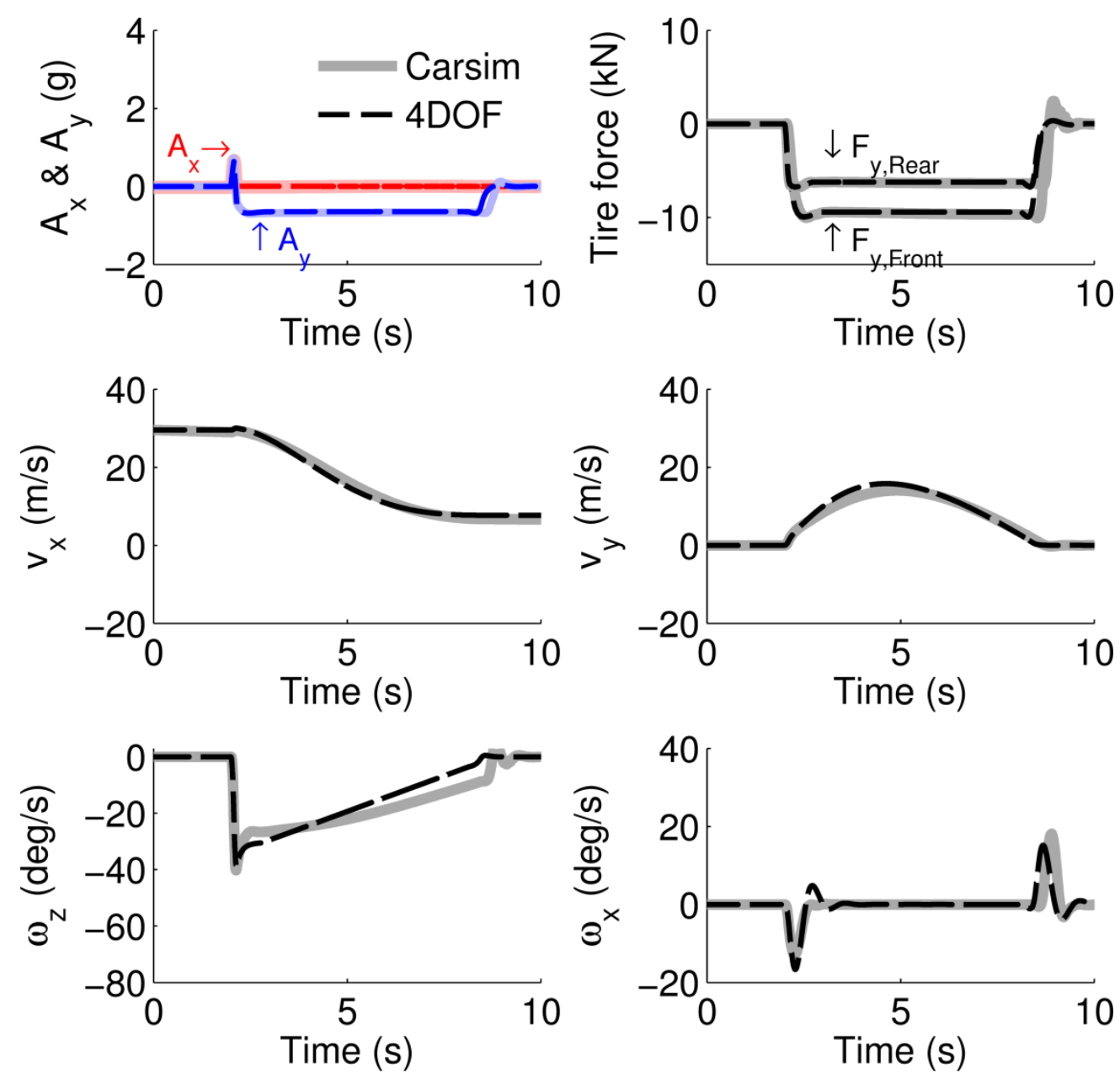

(b) Vehicle maneuver comparison

Figure 2.12 Vehicle response after an impact (No control action is involved. Initial conditions: $v_{x}=30 \mathrm{~m} / \mathrm{s}$, the impact generates $A_{x}=0.67 \mathrm{~g}, A_{y}=0.7 \mathrm{~g}$ ) 


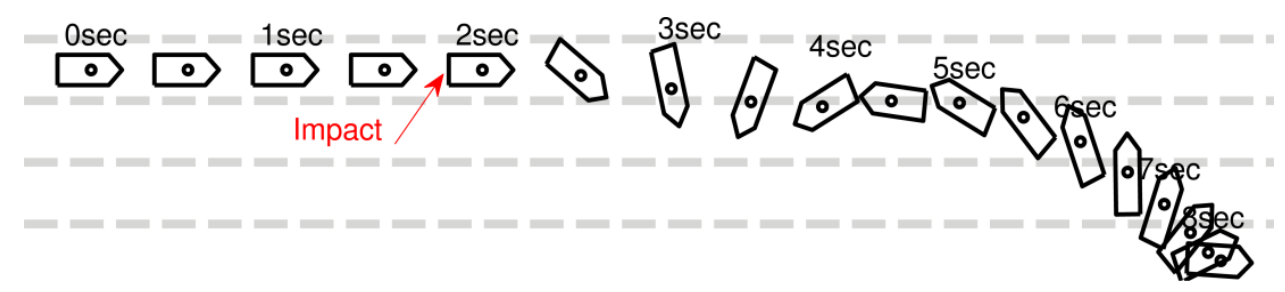

(a) Vehicle trajectories
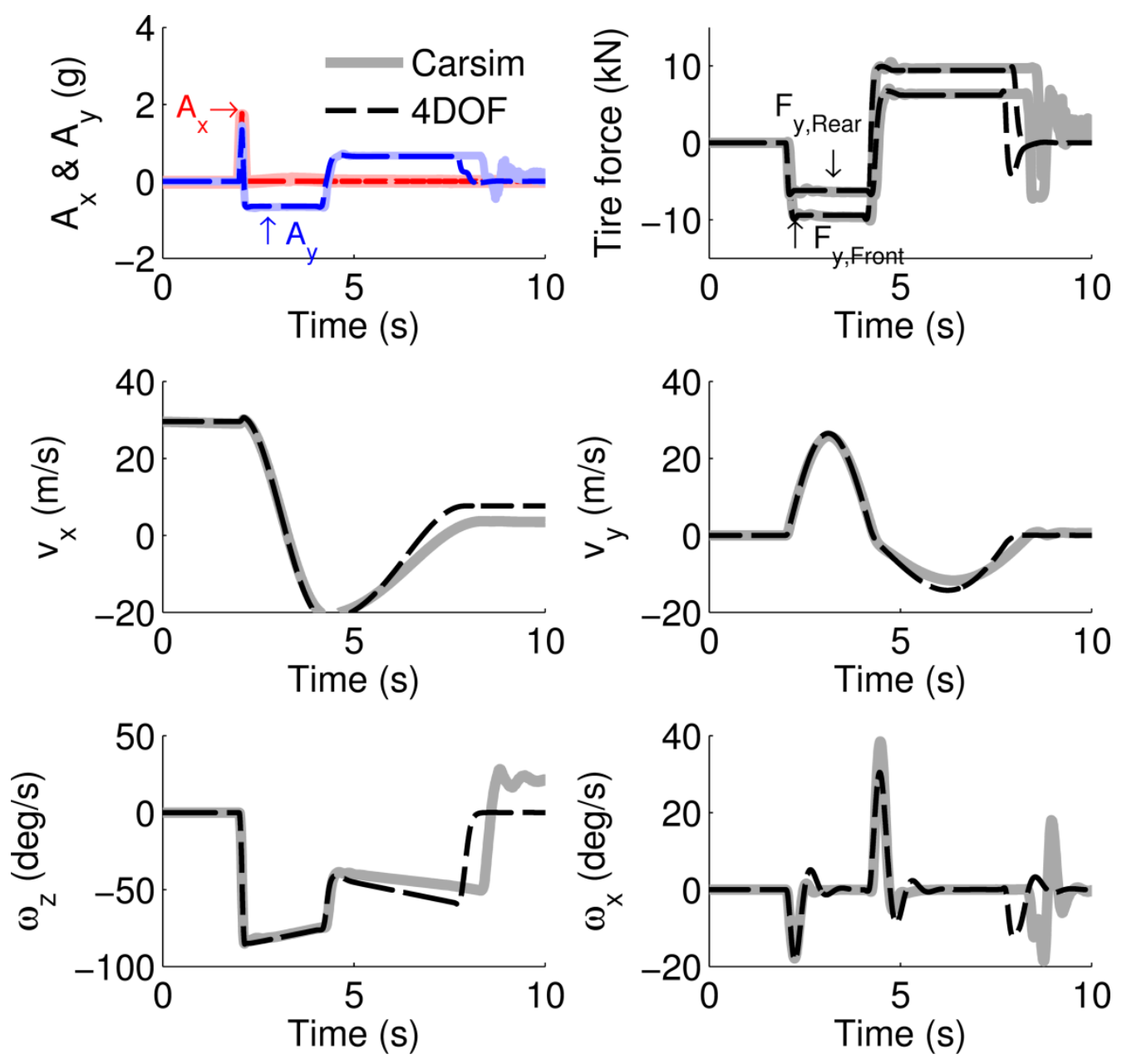

(b) Vehicle maneuver comparison

Figure 2.13 Vehicle response after an impact (No control action is involved. Initial conditions: $v_{x}=30 \mathrm{~m} / \mathrm{s}$, the impact generates $A_{x}=1.7 \mathrm{~g}, A_{y}=1.4 \mathrm{~g}$ ) 


\section{CHAPTER 3 \\ COLLISION STRENGTH ESTIMATION AND VEHICLE MOTION PREDICTION}

In this chapter, methods to estimate the strength of impact forces and predict vehicle response after an impact are proposed. The proper vehicle control actions will then be determined based on the predicted vehicle motion. The algorithm is designed based on a few assumptions. First, the entire event is assumed to occur on a straight road, and only two vehicles (a striking vehicle and a struck vehicle) are involved in the collision. The sensors and steering/braking actuators in the target vehicle are assumed to be intact after the collision and will function normally despite the collision.

A vehicle collision model to characterize the vehicle motions after a light impact is developed based on the research in [50]. Since the vehicle velocities and yaw rate change abruptly during the impact, the crash can be detected within a very short period of time during the crash using the yaw rate and lateral acceleration signals. Based on the law of conservation of momentum, the strength of the impact force and the impact location are estimated. The process for predicting the impact force is shown in Figure 3.1.

To achieve faster response, the crash force prediction is performed before reaching the half-way point of the crash duration. Measured signals from the vehicle sensors at each time step are used to estimate the impact impulses. Once a crash event is detected, the earliest three step points of the impulse estimations are used to project the half-way duration point over the entire collision pulse duration. A projection is obtained by using a linear extrapolation through previous estimation points. Then, impulse estimations in later steps keep correcting the projection point. Approximating the impact force by a triangular shape with a preset crash duration [58], the impulse strength can be

predicted at an early stage. The vehicle response after impact is then predicted using the 4-DOF vehicle equation of motion shown in Section 2.1.1. 


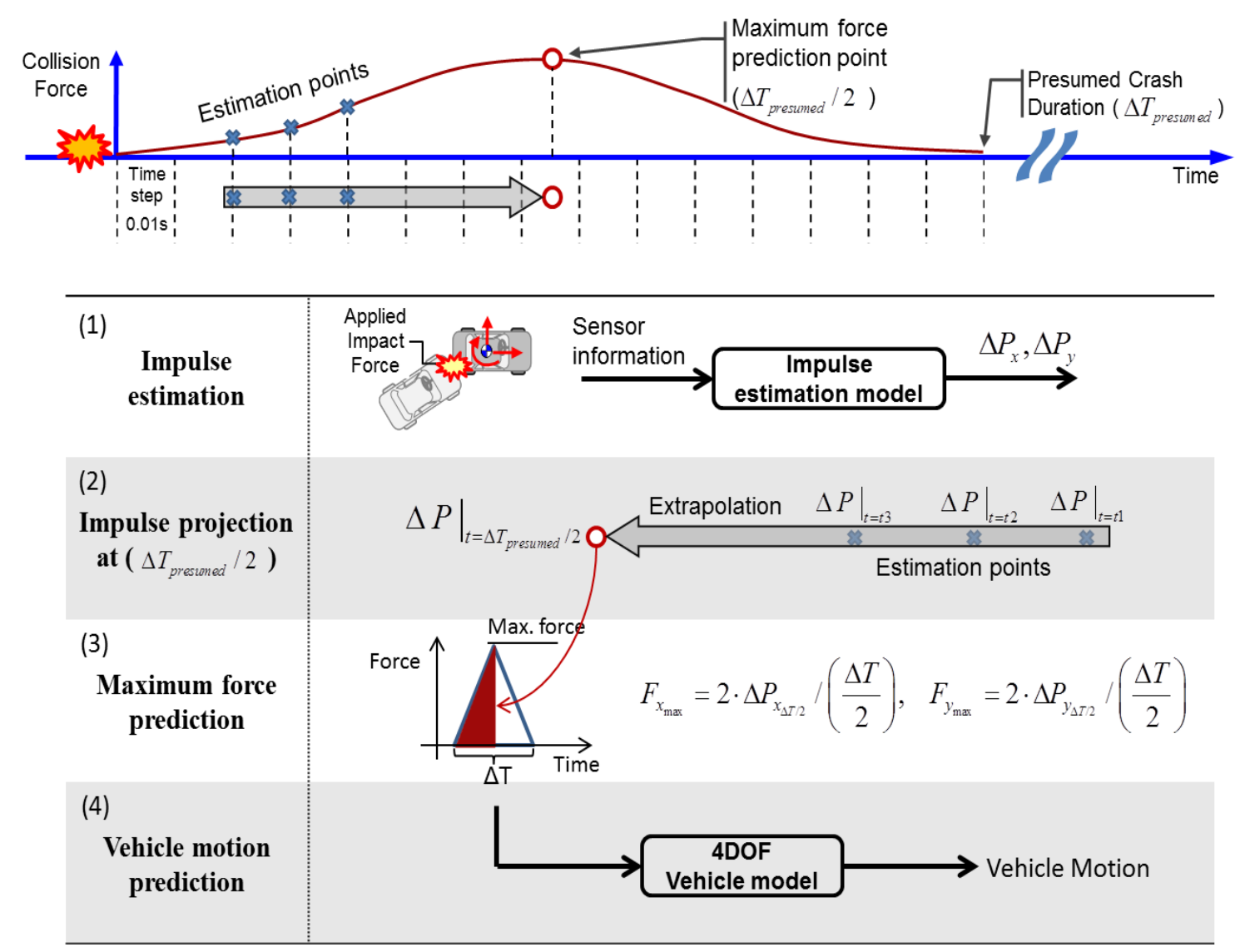

Figure 3.1 Function diagram for impact impulse and vehicle motion prediction

\subsection{Collision Force Profile and Time Duration}

Collision force profile represents the time history of the force magnitude during the collision. The collision force is characterized by shape, amplitude, and time duration. Various collision force shapes have been proposed to fit observed accelerometer signals in crash experiments [58, 59]. It was shown in [59] that different force shapes were observed depending on the collision types. The approximated shapes include haversine, sine wave, square, and triangular type. Among them, offset collisions result in vehicle lateral accelerations that are approximately triangular, as shown in Figure 3.2. Therefore, we assume the force shape is triangular because the offset collision induces more vehicle yaw and lateral motions, which is our interest in this research. If the triangle is isosceles, it is characterized by the base length and the height. This assumption makes the area 
(impulse strength) calculation easy: The area of the triangle is obtained by multiplying the base by the height, and then divided by 2 .

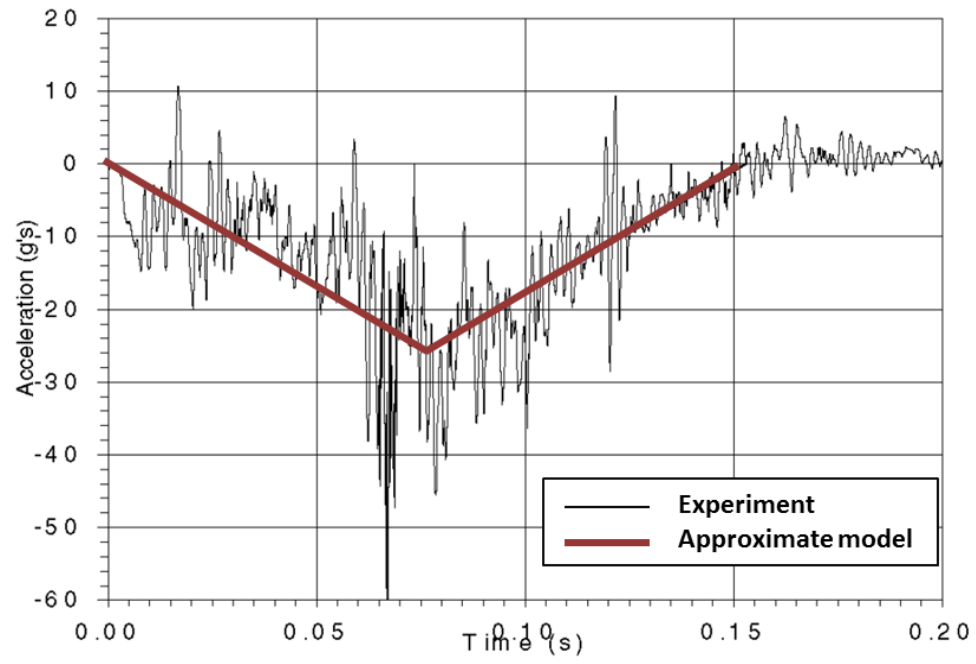

Figure 3.2 An example of acceleration measurement data from the load cell on a collision test vehicle and its approximation model [59]

As presented in [50], it is a good starting point to assume that the impulsive force lasts for 0.15 seconds. This time duration assumption may cause an error when predicting the total collision force magnitude when the actual duration is not exactly 0.15 seconds long. Although the variation of typical time duration for a collision is not wide (almost always between 0.1 and 0.2 seconds) as shown in Figure 3.3, the collision force prediction is still affected.

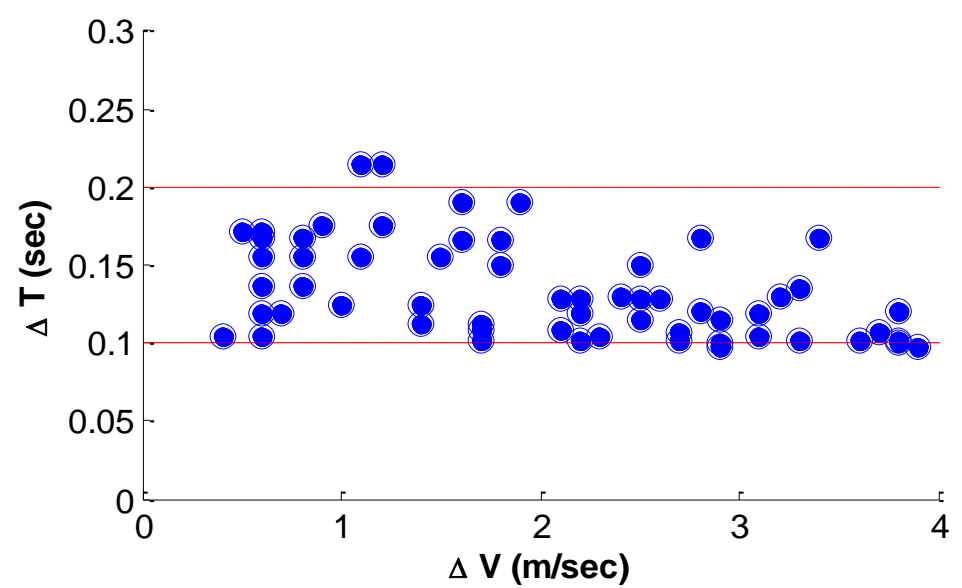

Figure 3.3 Collision time durations for 30 different car-to-car collision cases with various closing speed conditions ( $\Delta T$ : Time duration, $\Delta \mathrm{V}$ : Closing velocity) $[60,61]$ 
A triangle can be defined when we know two angles and the side length between the angles. In case of isosceles triangle which has two equal sides, only one angle is needed because both angles are the same. Taking this into account, the earliest three step points are used to determine the slope of a triangle, meaning the angle of the triangle. Then, the collision time duration is taken as the base of a triangle. The magnitude of the predicted maximum force varies depending on this collision time duration. Since the collision profile is predicted with presumed time duration ( 0.15 seconds $)$, the time duration needs to be updated for more accurate collision force prediction. Figure 3.4 demonstrates the different force predictions with triangles that have the same slopes but different time durations. It is obvious that the impulse strength error is linearly related to the impulse duration error. Hence, the presumed time duration assumption needs to be updated to correct the collision force strength. This correction process is performed during the collision process. Since the magnitude of the impulse is the time-integrated area of the collision force, the actual collision duration can be estimated from the changing rate of the impulse values.

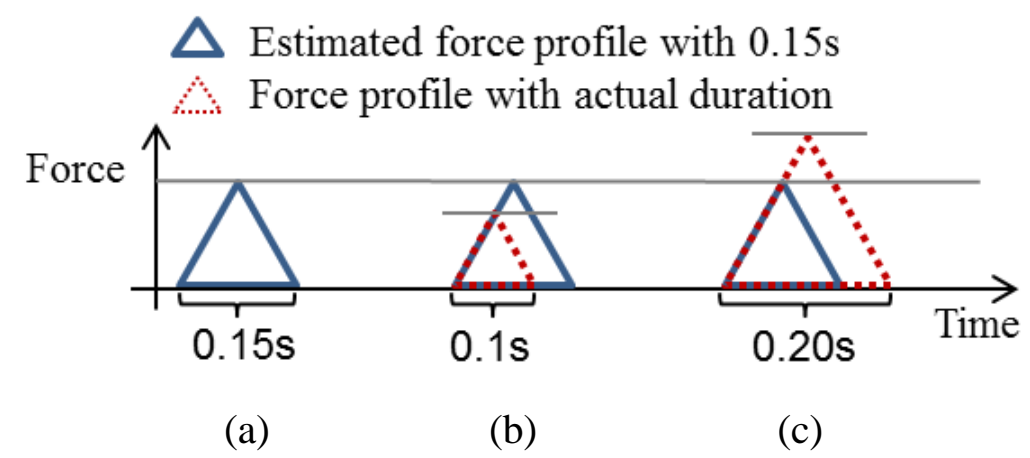

Figure 3.4 Maximum force levels with different crash time durations

If the collision ends earlier than the presumed time, there exists a point of time where the collision impulse does not increase before reaching the presumed time duration, 0.15 seconds. This indicates that the impulse reaches maximum level earlier and the collision ends around that time. For this reason, the collision force prediction algorithm keeps running even after the control is activated. If it is determined to be an over-prediction, as shown in Figure 3.4 (b), either the control magnitude should be reduced or the desired state should be revised, or both. To detect the maximum level of 
the impulse, two sampling steps are used to update the collision time duration. After detection, a new triangular collision profile with new time duration is constructed.

Similarly, if the collision ends later than the presumed time, the collision impulse keeps increasing even after passing through 0.15 seconds and the collision force is underpredicted, as shown in Figure 3.4 (c). In this case, the collision force prediction keeps updating with extended collision time durations until reaching saturation. Then, the updated force profile helps to either determine a beneficial desired state or increase the control magnitude.

\subsection{Impulse Estimation Model}

Using the vehicle sensor information, the magnitude and the location of the impulse can be computed by using a crash dynamics model and the measured vehicle states. The model includes the equations of motion in the longitudinal, lateral, and yaw degrees of freedom, presented in discrete time [50]. The strengths of the impulse $\left(\Delta P_{x}, \Delta P_{y}\right)$ and the location in the $x-y$ local coordinate plane $\left(x_{A}, y_{A}\right)$ are then inferred from the equations as follows:

$$
\begin{gathered}
\Delta P_{x}=m \cdot\left(v_{x, t}-v_{x, t-\Delta t}\right)-m \frac{\Delta t}{2}\left(v_{y, t} \cdot \omega_{z, t}+v_{y, t-\Delta t} \cdot \omega_{z, t-\Delta t}\right) \\
\Delta P_{y}=m \cdot\left(v_{y, t}-v_{y, t-\Delta t}\right)+m \frac{\Delta t}{2}\left(v_{x, t} \cdot \omega_{z, t}+v_{x, t-\Delta t} \cdot \omega_{z, t-\Delta t}\right) \\
-m_{R} h \cdot\left(\omega_{x, t}-\omega_{x, t-\Delta t}\right)-\frac{\Delta t}{2} C_{f}\left(\frac{v_{y, t}+a \cdot \omega_{z, t}}{v_{x, t}}+\frac{v_{y, t-\Delta t}+a \cdot \omega_{z, t-\Delta t}}{v_{x, t-\Delta t}}\right) \\
-\frac{\Delta t}{2} C_{r}\left(\frac{v_{y, t}-b \cdot \omega_{z, t}}{v_{x, t}}+\frac{v_{y, t-\Delta t}-b \cdot \omega_{z, t-\Delta t}}{v_{x, t-\Delta t}}\right) \\
\Delta P_{y} x_{A}-\Delta P_{x} y_{A}=I_{z z}\left(\omega_{z, t}-\omega_{z, t-\Delta t}\right)+I_{x z}\left(\omega_{x, t}-\omega_{x, t-\Delta t}\right) \\
-\frac{\Delta t}{2} a C_{f}\left(\frac{v_{y, t}+a \cdot \omega_{z, t}}{v_{x, t}}+\frac{v_{y, t-\Delta t}+a \cdot \omega_{z, t-\Delta t}}{v_{x, t-\Delta t}}\right) \\
+\frac{\Delta t}{2} b C_{r}\left(\frac{v_{y, t}-b \cdot \omega_{z, t}}{v_{x, t}}+\frac{v_{y, t-\Delta t}-b \cdot \omega_{z, t-\Delta t}}{v_{x, t-\Delta t}}\right)
\end{gathered}
$$


The above equations are derived by integrating Equations (2.1)-(2.3), given that the collision impulse is an integration of collision forces $\left(\Delta P_{i}=\int_{t 1}^{t 2} F_{i} d t, i=x, y\right)$. For the integration of nonlinear terms $\left(v_{y} \omega_{z}, v_{x} \omega_{z}, v_{y} / v_{x}, \omega_{z} / v_{x}\right)$, the trapezoidal rule shown in Figure 3.5 is used with a fixed time step $(\Delta t)$. The variables on the right-hand sides of Equations (3.1)-(3.3) are all assumed to be known from either the vehicle sensor measurements or the vehicle parameters. The subscripts for time ( $t$ and $t-\Delta t$ ) indicate measured values at each different time step. For the cornering stiffness $\left(C_{\alpha}\right)$, the time varying values are used based on the tire slip angles. Since the collision impact dramatically changes the slip angles and the lateral forces on tires, the effective factor for cornering stiffness in Equation (2.23) is updated and applied throughout the impulse.

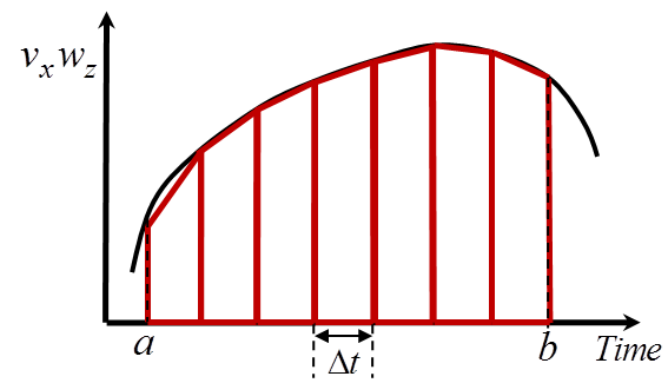

Figure 3.5 Illustration of the trapezoidal rule showing a function (black line) integration is approximated by trapezoids (red lines)

Note that the model has more unknowns $\left(\Delta P_{x}, \Delta P_{y}, x_{A}\right.$, and $\left.y_{A}\right)$ than the number of equations. To solve four unknown variables based on three equations, we use the assumption that the collision event occurs either on the sides or at the rear bumper of the vehicle. As shown in Figure 3.6, if a side impact occurs, the longitudinal location of impact $x_{A}$ is unknown, but the lateral position of impact $y_{A}$ should be equal to $y_{a}$. Similarly, in the case of rear-end impact, the longitudinal position of impact $x_{A}$ should be equal to $x_{a}$. Considering the fact that $x_{a}$ is the distance between CG and the rear bumper and $y_{a}$ is the half vehicle width, the calculated $x_{A}$ and $y_{A}$ values should be within the vehicle geometric boundaries. Consequently, when solving Equation (3.3) for $x_{A}$ and $y_{A}$, two cases (side and rear-end impact assumptions) will be calculated. Among them, only solutions with geometrically realistic values that do not exceed the values of $x_{a}$ and $y_{a}$ will be chosen, as shown in [5]. 


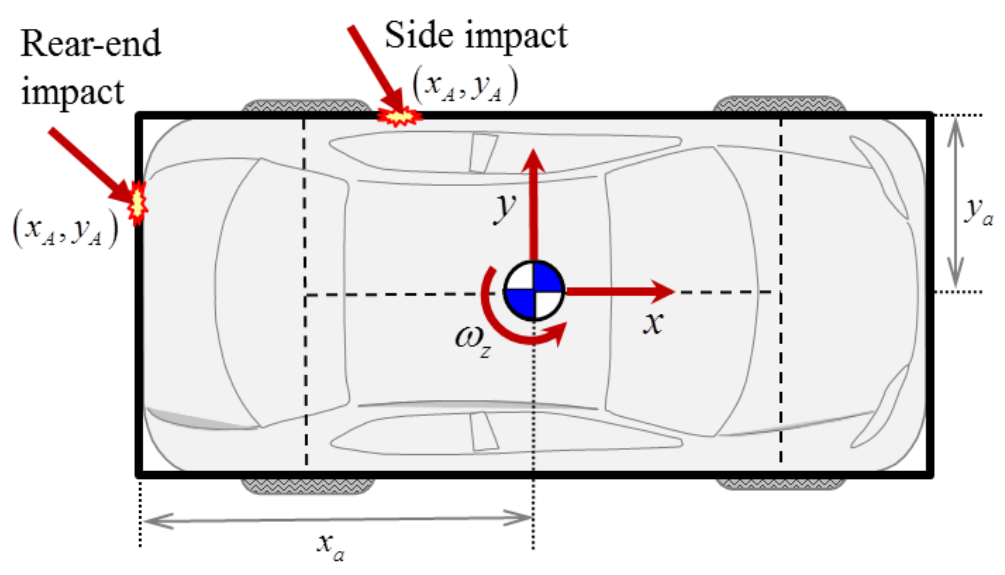

Figure 3.6 Impact locations on vehicle periphery in a collision [5]

\subsection{Impact Force Prediction}

It is desired that the collision force should be determined at an early stage of collision process. Early prediction information allows the controller (which will be described in Chapter 5) to perform promptly. For this reason, the estimated collision impulses $\left(\Delta P_{x}, \Delta P_{y}\right)$ are used to project the impulse at future time step by linear extrapolation. The projected point is half of presumed crash duration $(\Delta T / 2)$. Since the impact force profile is assumed to be an isosceles triangle, the projected impulse at halfway duration point will be half of the area of the triangle. Using the projected impulses, the maximum forces $\left(F_{x_{\max }}, F_{y_{\max }}\right)$ are then calculated by evaluating the maximum height of the triangle profile with the following relationships:

$$
F_{x_{\max }}=2 \cdot \Delta P_{x_{\Delta T / 2}} /\left(\frac{\Delta T}{2}\right), \quad F_{y_{\max }}=2 \cdot \Delta P_{y_{\Delta T / 2}} /\left(\frac{\Delta T}{2}\right)
$$

Once the maximum forces are determined from Equation (3.4), the entire collision force profiles can be constructed based on the presumed crash duration as shown in Figure 3.7. Because the apex of the isosceles triangle is directly above the base's midpoint, the maximum force level and the collision time duration are enough to describe the collision force prediction. This information will be fed into the algorithm that predicts the expected vehicle motion with the collision forces. 


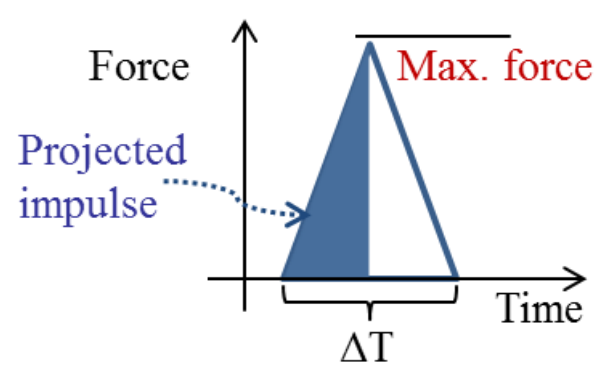

Figure 3.7 Trianglular collision profile showing the relationship between the maximum force level and the projected impulse

Still, as mentioned in Section 3.1, the collision force profiles need to be updated with the corrected time duration. There are two possible cases as demonstrated in Figure 3.8: (a) Under-estimation case with $\Delta T_{\text {actual }}>\Delta T_{\text {presumed }}$, and (b) Over-estimation case when $\Delta T_{\text {actual }}<\Delta T_{\text {presumed }}$.

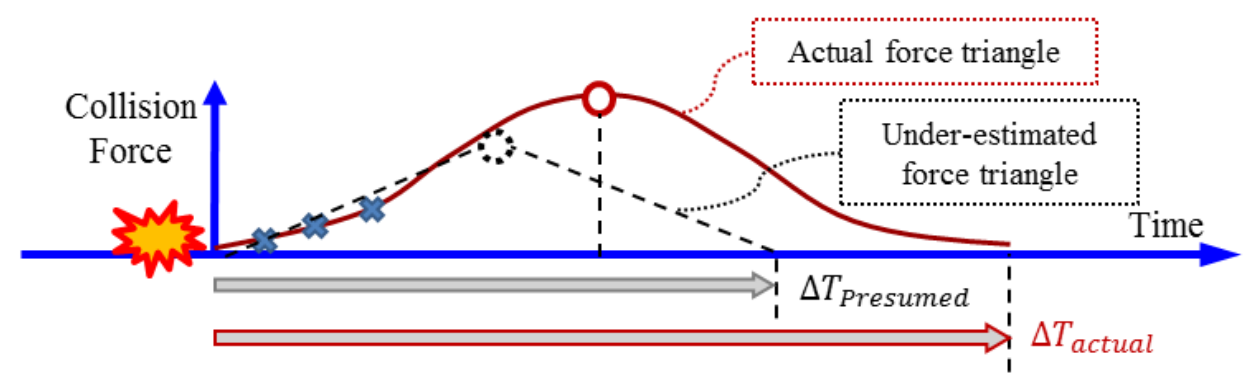

(a) $\Delta \mathrm{T}_{\text {actual }}>\Delta \mathrm{T}_{\text {Presumed }}$, Under-estimation case

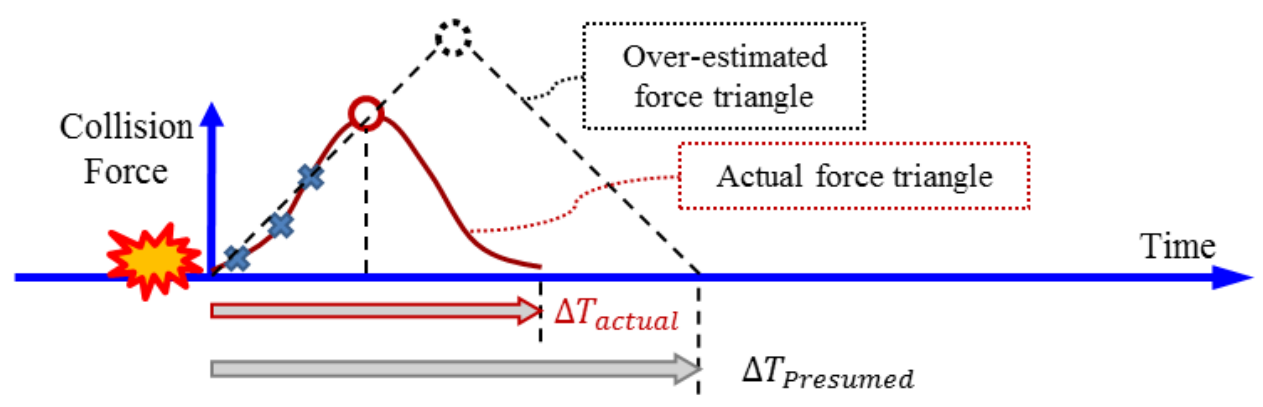

(b) $\Delta T_{\text {actual }}<\Delta T_{\text {Presumed }}$, Over-estimation case

Figure 3.8 Under-estimation and over-estimation cases depending on the actual collision time durations (Crosses indicate impulse estimation points at each timestep, and circles indicate the peak point of each profile) 
Recalling that the size of the triangle can be determined by the integration of impulse estimations, it is important to consider the behavior of the estimated impulse curve. In order to determine the cases that the time duration is not close to the presumed time, the inflection point of the impulse curve is considered. At the inflection point, the curvature changes from positive to negative or vice versa. So, the inflection point can be found by checking the sign of the second derivative of the function:

$$
\left(\left.\frac{d^{2} f}{d t^{2}}\right|_{t-\Delta t}>\left.0 \quad \& \quad \frac{d^{2} f}{d t^{2}}\right|_{t}<0\right)
$$

where, $f$ is the output curve corresponding to a time $t$, denoted by $f(t)$.

Figure 3.9 demonstrates an example result showing the algorithm finding an inflection point on the estimated impulse curve at which the actual time duration is longer than the presumed value ( $\Delta T_{\text {actual }}=0.2$ seconds and $\Delta T_{\text {Presumed }}=0.15$ seconds). The location of the green bar in (a) is the point where the inflection appears on the curve (at $\mathrm{t}=2.1$ seconds). The impulse estimation continues until this inflection point is recognized, as shown by the blue dashed line. Because this inflection point is considered as the halfway point of the actual time duration of the force profile, the force prediction can simultaneously be acquired at the point based on Equation (3.4).

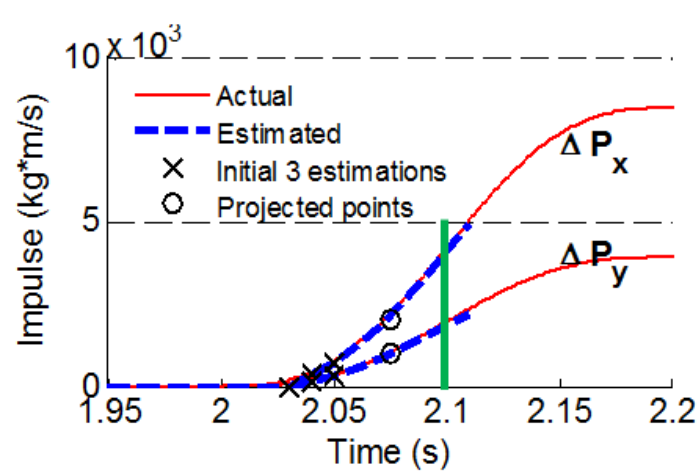

(a) Impulses

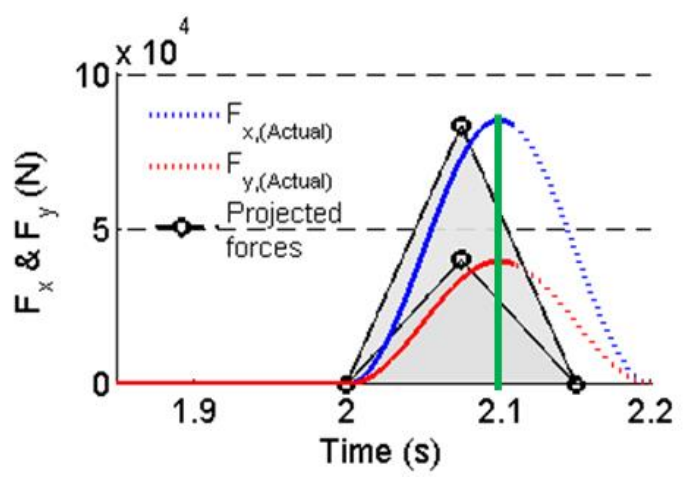

(b) Collision forces

Figure 3.9 Collision impulse and force estimation where the actual crash duration is 0.2 seconds while the presumed duration is 0.15 seconds. (The green bars indicate the time when the algorithm can detect the inflection point.) 


\subsection{Simulation Results for Impact Force Prediction}

The proposed impulse estimation and vehicle motion prediction algorithms are validated using Carsim simulations as shown in Figure 3.10. The impact occurs at 2.0 seconds and the haversine-shaped external forces are applied to the simulation model. The impulse is estimated using the measured sensor signals and the equations of motion (3.1)-(3.3). The linearly extrapolated results project the impulse at the half-way point of the crash duration. Estimated impulse points are marked with crosses and the crash event is first detected at 2.04 seconds.

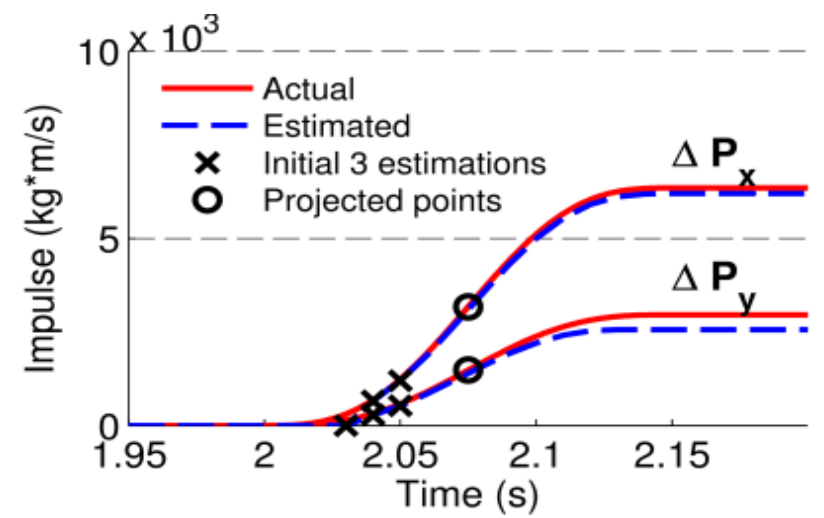

(a)

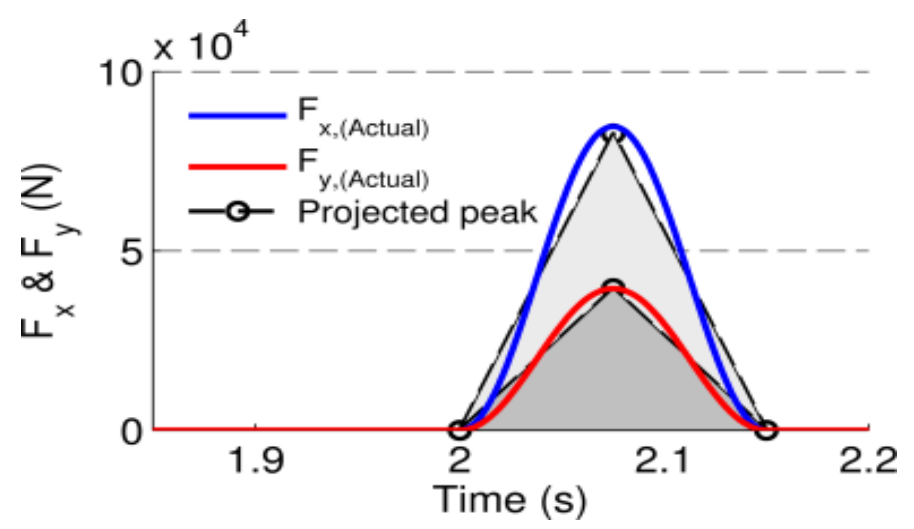

(b)

Figure 3.10 Comparison of the actual and projected impulse and impact forces ('o' represents the projection point of the maximum forces at $\Delta T=0.15$ seconds)

(a) Collision impulses, (b) Collision forces

Since the collision duration $(\Delta T)$ is initially assumed to be 0.15 seconds, the projected impulse is marked at 2.075 seconds (half of the duration). The predicted forces are obtained by calculating the height of the assumed triangle with an area that is twice 
the size of the projected impulse magnitude. The result shows that the projected triangle force shapes are fairly close to the actual ones.

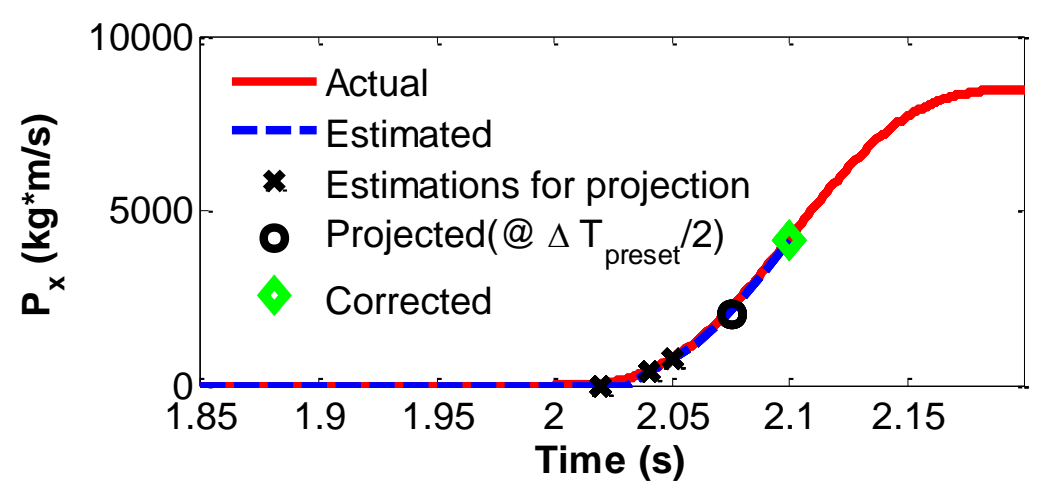

(a) Collision impulse profiles

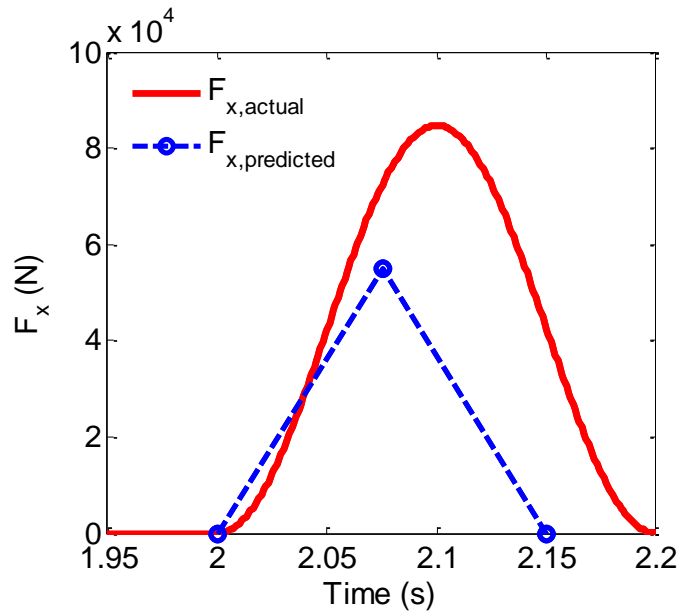

(b) Prediction before the inflection point

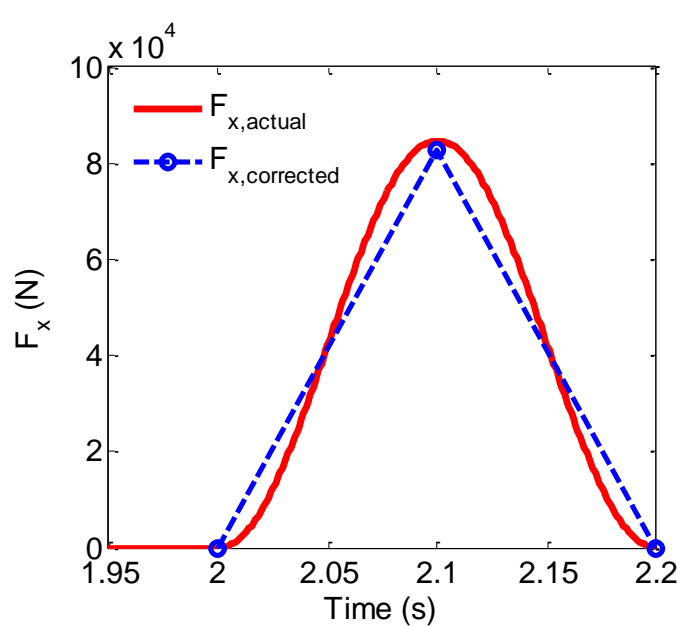

(c) Prediction after the inflection point

Figure 3.11 Collision prediction in a case when the actual collision time duration ( $\Delta T=0.2$ seconds) is longer than the nominal value

To check the performance of the prediction with respect to collision time duration, several crash time durations are simulated. Figure 3.11 and Figure 3.12 demonstrate the corrections of the projected impulses and different force predictions depending on collision time durations. Figure 3.11 (a) shows that the estimated impulse keeps increasing even after the initially projected point $\left(\Delta T_{\text {Presumed }} / 2=0.075\right.$ seconds $)$. The prediction keeps updating until an inflection point is found. On the other hand, Figure 3.12 (a) shows the inflection point is found even before the initially projected point ( 0.075 seconds). The algorithm recognizes the actual duration is shorter, and a new 
collision force profile is generated using the corrected time duration ( 0.1 seconds). Figure 3.11 (c) and Figure 3.12 (c) show the new triangular collision profiles with the corrected time duration.

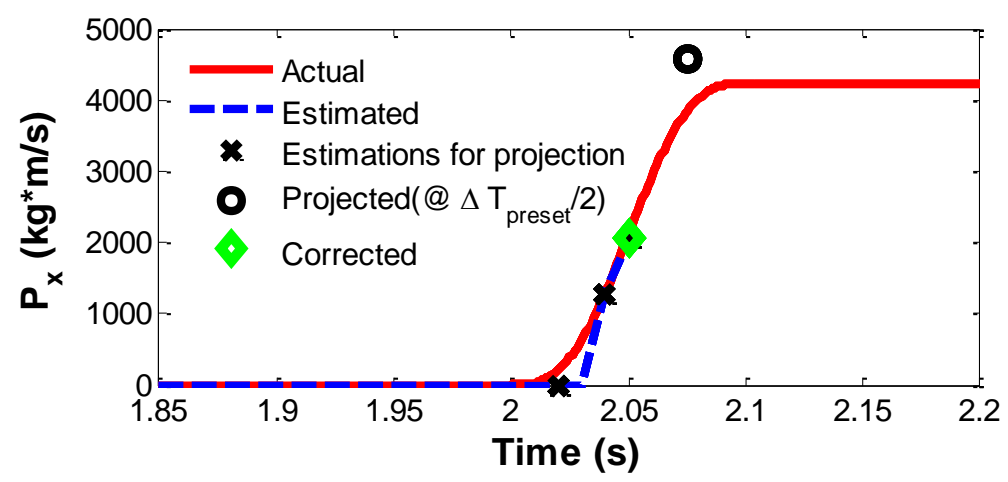

(a) Collision impulse profiles

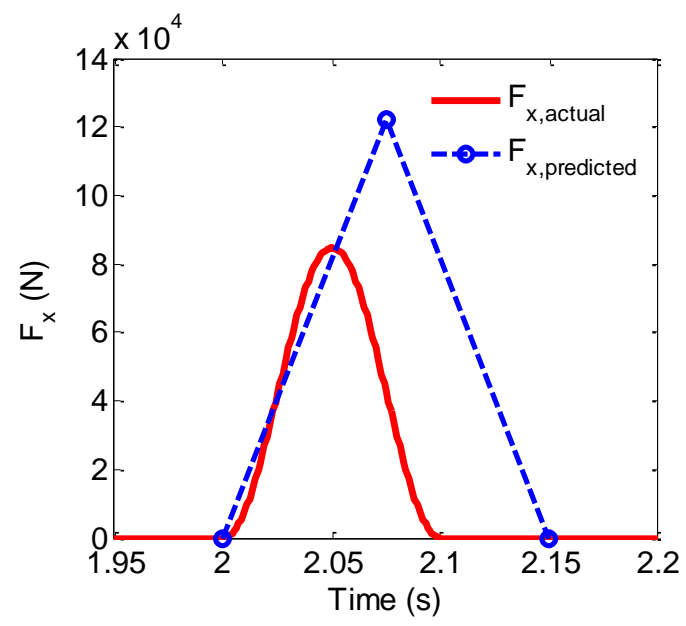

(b) Prediction before the inflection point

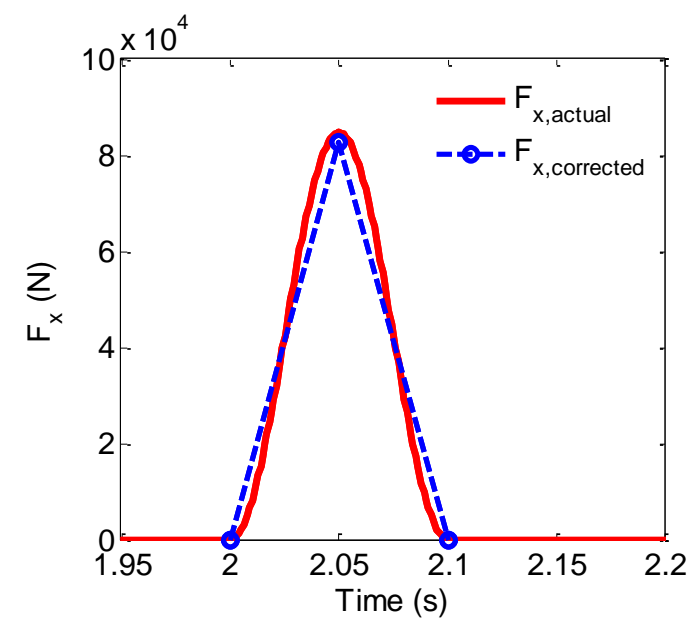

(c) Prediction after the inflection point

Figure 3.12 Collision prediction in a case when the actual collision time duration ( $\Delta T=0.1$ seconds) is shorter than the nominal value

To quantitatively compare the actual and predicted forces, the areas under the force curves are examined. Since the magnitudes of the predicted impulses are obtained by integrating the force profile, comparing the areas of the collision force profiles to evaluate the performance of the proposed algorithm is reasonable. Figure 3.13 shows the force prediction area error along the range of collision time durations from 0.1 seconds to 0.2 seconds. Note that the error levels in the longitudinal collision forces $\left(F_{x}\right)$ are similar, while the lateral collision error levels are more variable. One of the reasons for 
this difference is the effect of tire force variations. Unlike the longitudinal impulse $\left(\Delta P_{x}\right)$, the equation for solving the lateral impulse $\left(\Delta P_{y}\right)$ has terms relating to tire forces, as shown in Equation (3.2). Therefore, the prediction performances are affected by the tire force errors. The overall area errors between the actual and the predicted forces were found to be less than $10 \%$ and on average the error is around $5 \%$.

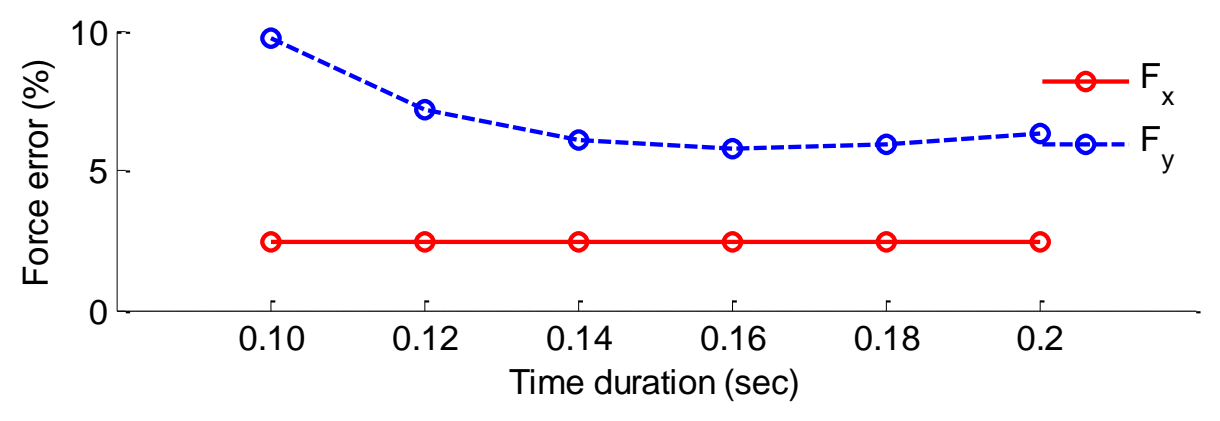

Figure 3.13 Predicted force errors

The proposed algorithm is also validated with real collision experimental data from the NHTSA Crash Test Database [62]. There are four major test scenarios in this database: (1) frontal offset test, (2) side impact test, (3) pedestrian test, and (4) pole test. The frontal offset tests were chosen for the validation. Although the frontal offset crash test is not exactly the same angled side impacts, the crash type is similar. An example test scene is shown in Figure 3.14. Moreover, although the speed differences between the two cars are relatively high, the test data can still be used to evaluate the collision force estimation method.

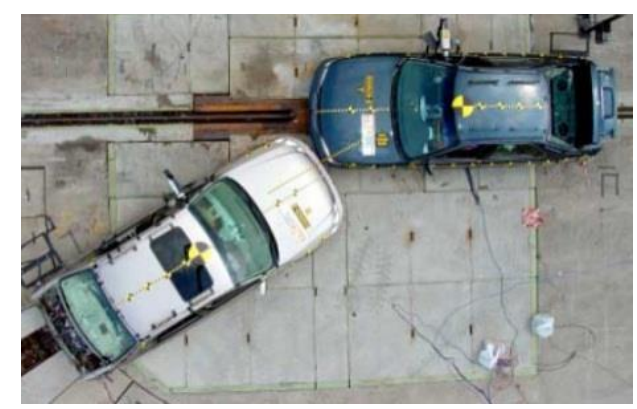

Figure 3.14 An oblique frontal offset crash test scene in the NHTSA Crash Test [62]

Validation results using four different experimental data sets are shown in Figure 3.15. Similar to the results shown in Figure 3.10, the maximum collision forces are 
projected and marked with the circles and the triangle symbols are the projected collision profiles. The error of the force profiles are shown in Table 3.1. Overall, the projected force areas are fairly close to the actual force areas except the case in Figure 3.15 (d). The lateral collision force in this result shows $26 \%$ error because the collision estimation algorithm misses a large peak of the force.

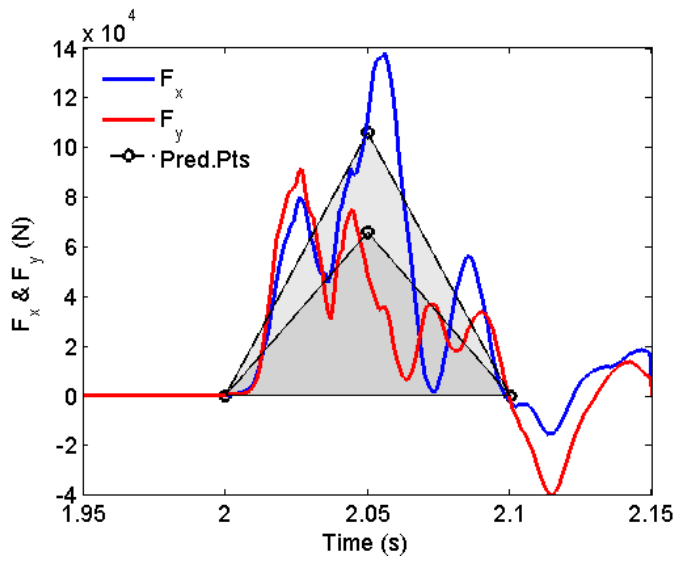

(a)

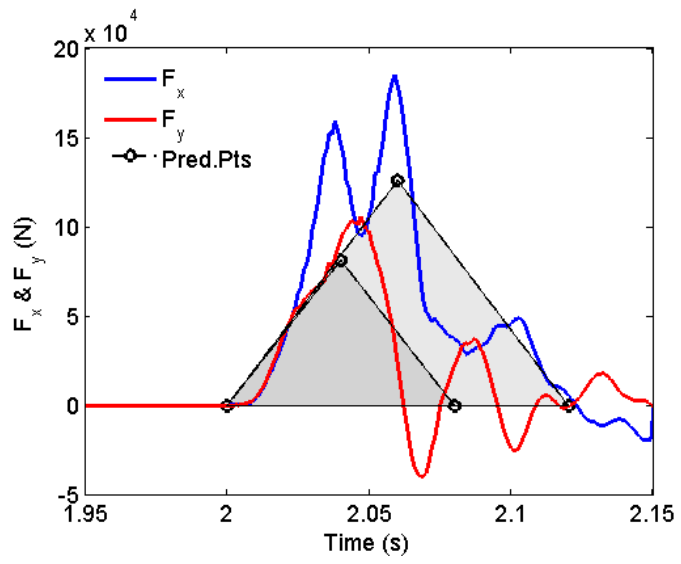

(c)

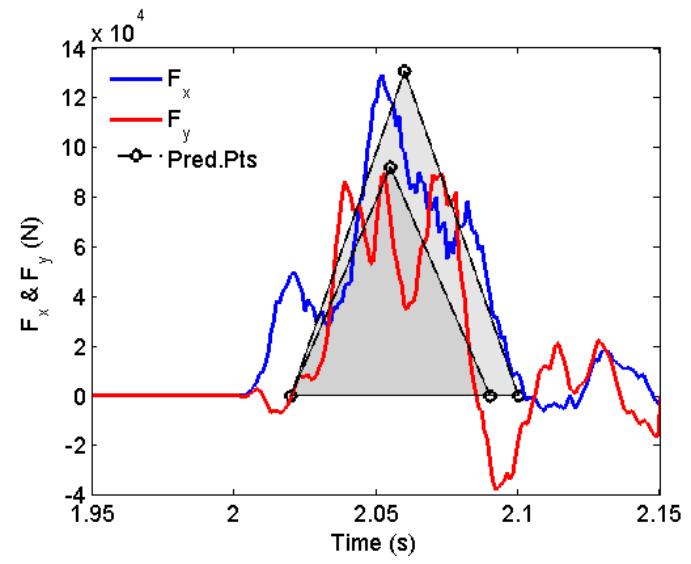

(b)

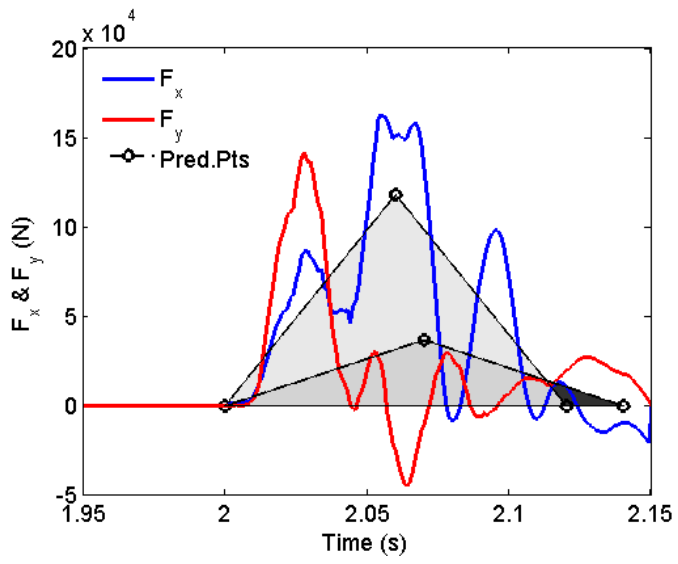

(d)

Figure 3.15 Validation of the prediction algorithm using a real crash data ('o' represents the projection point of the maximum forces, the gray lines represents the triangular shape of the predicted forces)

Table 3.1 Area errors of the collision force prediction algorithm

\begin{tabular}{|c|c|c|c|c|}
\hline Forces & (a) & (b) & (c) & (d) \\
\hline$F_{x}$ & $1.5 \%$ & $7.2 \%$ & $1.9 \%$ & $2.5 \%$ \\
\hline$F_{y}$ & $4.2 \%$ & $4.1 \%$ & $4.1 \%$ & $26 \%$ \\
\hline
\end{tabular}




\subsection{Vehicle Motion Prediction}

The calculated impact forces are used to predict the vehicle motion. The 4-DOF vehicle dynamics model in [50] is used.

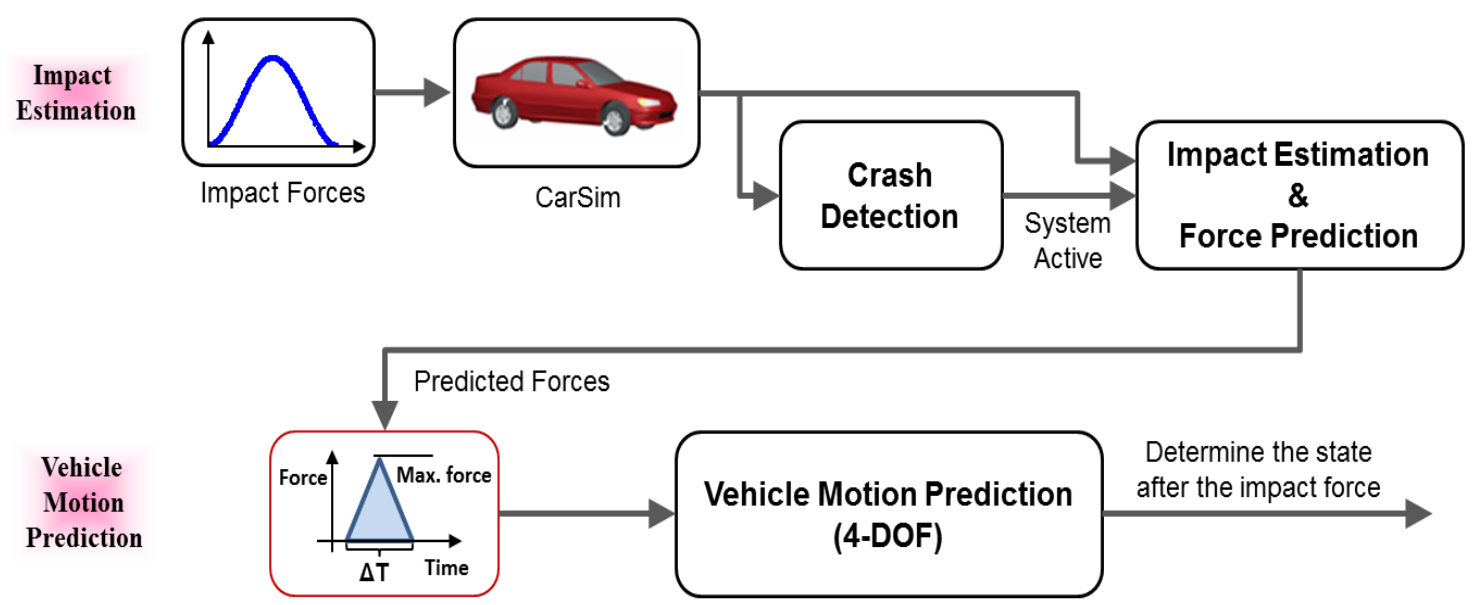

Figure 3.16 Block diagram of the collision impact estimation and the motion prediction model

A block diagram for the overall structure for collision impact estimation and vehicle motion prediction is presented in Figure 3.16. It consists of two major parts: impact estimation and motion prediction. The vehicle dynamics model in the form of a CarSim S-function takes external impact forces that have a haversine profile. The estimation starts when an impact is detected at the 'Crash Detection' block. The impact detection is performed by thresholds that indicate substantial changes in yaw rate and lateral acceleration. The thresholds are based on changes of yaw rate $\left(\omega_{z}\right)$ and lateral acceleration $\left(A_{y}\right)$ in successive sampling times ( 0.01 seconds interval), and its values are $\Delta \omega_{z}=3 \mathrm{deg} / \mathrm{s}$ and $\Delta A_{y}=0.1 \mathrm{~g}$. To increase the reliability of sensing information, three consecutive changes are considered. Crash detection generates a flag that enables the 'Impact Estimation \& Force Prediction' block. If the crash detection is valid, the impulse estimation is performed. The predicted force is fed into the 4-DOF vehicle model to determine the vehicle motion after the impact. 


\subsection{Simulation Results for Vehicle Motion Prediction}

To evaluate the effectiveness of the proposed estimation algorithm, a simulation is performed. Vehicle motion from CarSim is taken as the reference and compared with the prediction results from the 4-DOF model.

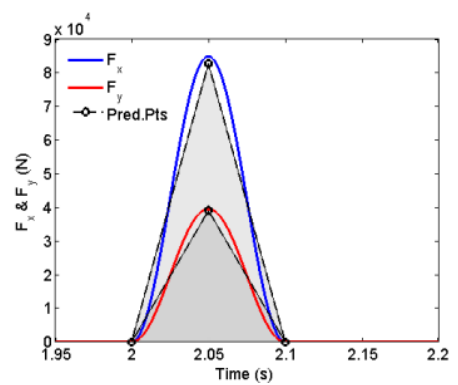

(a) $\Delta T=0.1$ seconds

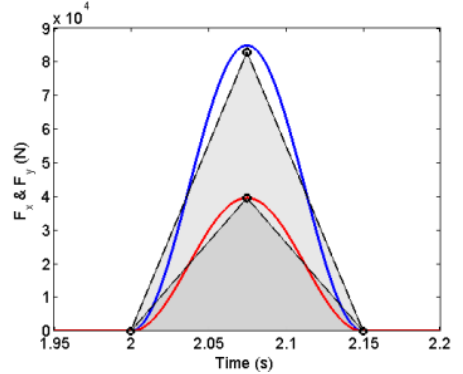

(b) $\Delta T=0.15$ seconds

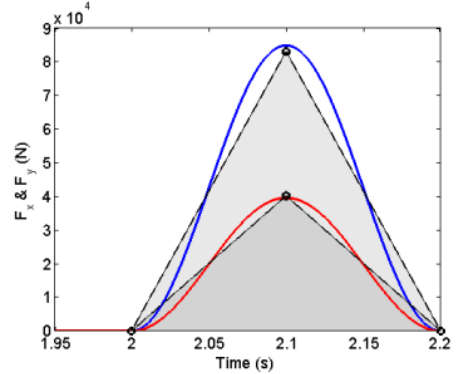

(c) $\Delta T=0.2$ seconds

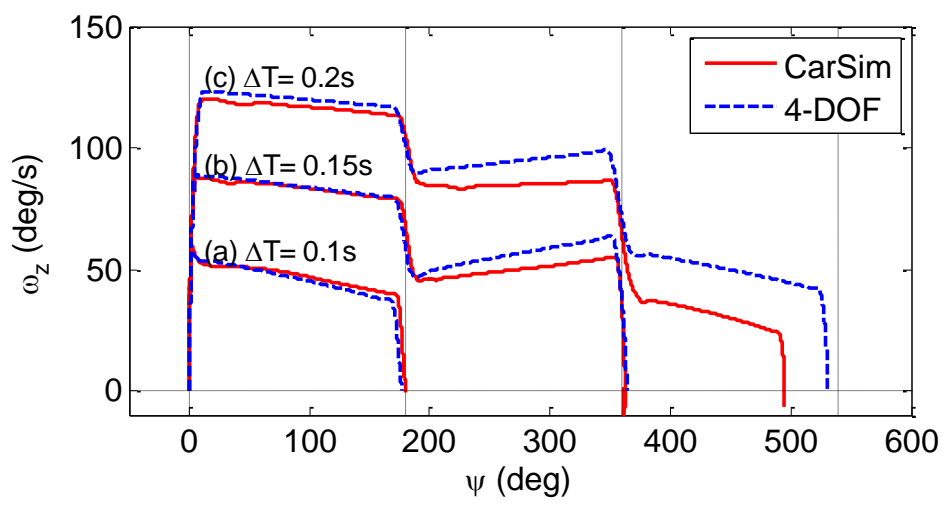

Figure 3.17 Vehicle motion prediction results with different durations of collision forces

Comparison between the CarSim simulation and the estimation is presented in Figure 3.17. Before the impact, the vehicle travels straight at $30 \mathrm{~m} / \mathrm{s}(67 \mathrm{mph}, 108 \mathrm{kph})$ with zero heading angle and yaw rate on a road surface with an adhesion coefficient of $\mu=0.7$. The impact is assumed to be located at $0.1 \mathrm{~m}$ to the right on the rear bumper. Three different collision forces are applied with varying collision time durations. The results show that the longer duration leads to more rotational motion with higher collision impulse. This also implies the importance of estimating the collision time duration for vehicle motion prediction. Although the discrepancies between CarSim and the 4-DOF model increase when the vehicle spins more with a higher yaw rate, responses within the 180 degree heading angle region show close agreement. 


\section{CHAPTER 4 DESIRED VEHICLE MOTION DETERMINATION}

In this chapter, the desired vehicle motion to mitigate secondary collision accidents is discussed. Before determining the desired vehicle motion, it is necessary to understand the governing dynamics in extreme vehicle maneuvers after an impact. The main analysis tool used in this chapter is the phase plane method [63-66]. Then, an optimization technique is used to determine the desired vehicle motion.

\subsection{Vehicle States in the Phase Plane}

The phase plane illustrates a system's dynamics graphically by plotting the state variables in a two-dimensional figure. This is a useful tool to visualize the behavior of nonlinear systems whose analytical solutions cannot be solved explicitly. The phase plane provides insight to the effect of initial states on the system, illustrating the state trajectory starting from different initial conditions. Showing the location of equilibrium points and regions of convergence is a useful way to analyze the nonlinear system behavior. In the vehicle dynamics field, phase plane analysis has been conducted to study vehicle motion of active steering control and ESC systems [63, 64]. Because the phase plane shows the system response, such as side slip angle, slip angle rate, and yaw rate, vehicle dynamic characteristics are easily visualized.

Figure 4.1 shows the phase plane plot of the vehicle side slip angle $(\beta)$ and yaw rate $\left(\omega_{z}\right)$. Assuming that the vehicle longitudinal speed $\left(v_{x}\right)$ is fixed at $30 \mathrm{~m} / \mathrm{s}(67 \mathrm{mph}$, $108 \mathrm{kph}$ ) and steering is zero, the phase plot is obtained by solving the two-wheel nonlinear planar bicycle model shown in Equations (4.1) and (4.2) with a series of initial conditions. The vehicle model is simplifications of Equations (2.2) and (2.3) considering only planar behavior of the vehicle without tire longitudinal forces. 


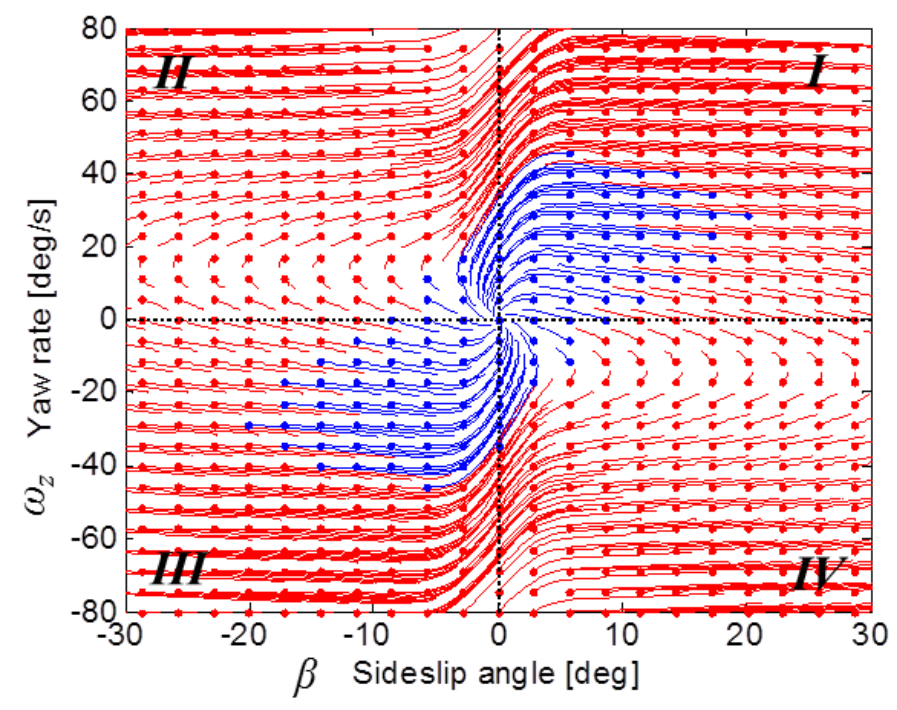

Figure 4.1 Vehicle dynamic motion analysis using the yaw rate-sideslip angle phase plane plot. Blue lines: converged in 1 second, Red lines: not converged in 1 second

(Convergence criteria: $\omega_{z} \in\left[-3.0^{\circ} / \mathrm{s},+3.0^{\circ} / \mathrm{s}\right], \beta \in\left[-1.5^{\circ},+1.5^{\circ}\right]$ )

$$
\begin{gathered}
m \cdot\left(\dot{v}_{y}+v_{x} \cdot \omega_{z}\right)=F_{y, f}+F_{y, r} \\
I_{z z} \cdot \dot{\omega}_{z}=a \cdot F_{y, f}-b \cdot F_{y, r}
\end{gathered}
$$

Initial impact effects are considered as non-zero initial states. Each nonlinear front and rear tire forces are summed as single equivalent tire forces on each axle.

$$
\begin{gathered}
F_{y, f}=F_{y, f_{L}}+F_{y, f_{R}} \\
F_{y, r}=F_{y, r_{L}}+F_{y, r_{R}}
\end{gathered}
$$

The vehicle side slip angle is defined as

$$
\beta=\arctan \left(\frac{v_{y}}{v_{x}}\right)
$$

The abscissa shows vehicle side slip angle and the ordinate shows the yaw rate. Each dot in Figure 4.1 represents an initial condition of the vehicle and the lines starting from the dots are traces of vehicle motion as they evolve over time. Here, the initial states, depicted as dots, are states caused by external impulsive disturbances. Then, the lines 
show the subsequent vehicle motion. Lines from each initial point, converging around the origin point $\left(\omega_{z} \in\left[-3.0^{\circ} / \mathrm{s},+3.0^{\circ} \mathrm{s}\right], \beta \in\left[-1.5^{\circ},+1.5^{\circ}\right]\right)$ within a selected time duration (for 1 second), are colored in blue. Other lines, colored in red, do not converge to the selected convergence region. The shape and the size of the convergence region change with steering angle, vehicle longitudinal velocity, road conditions, vehicle parameters, etc.

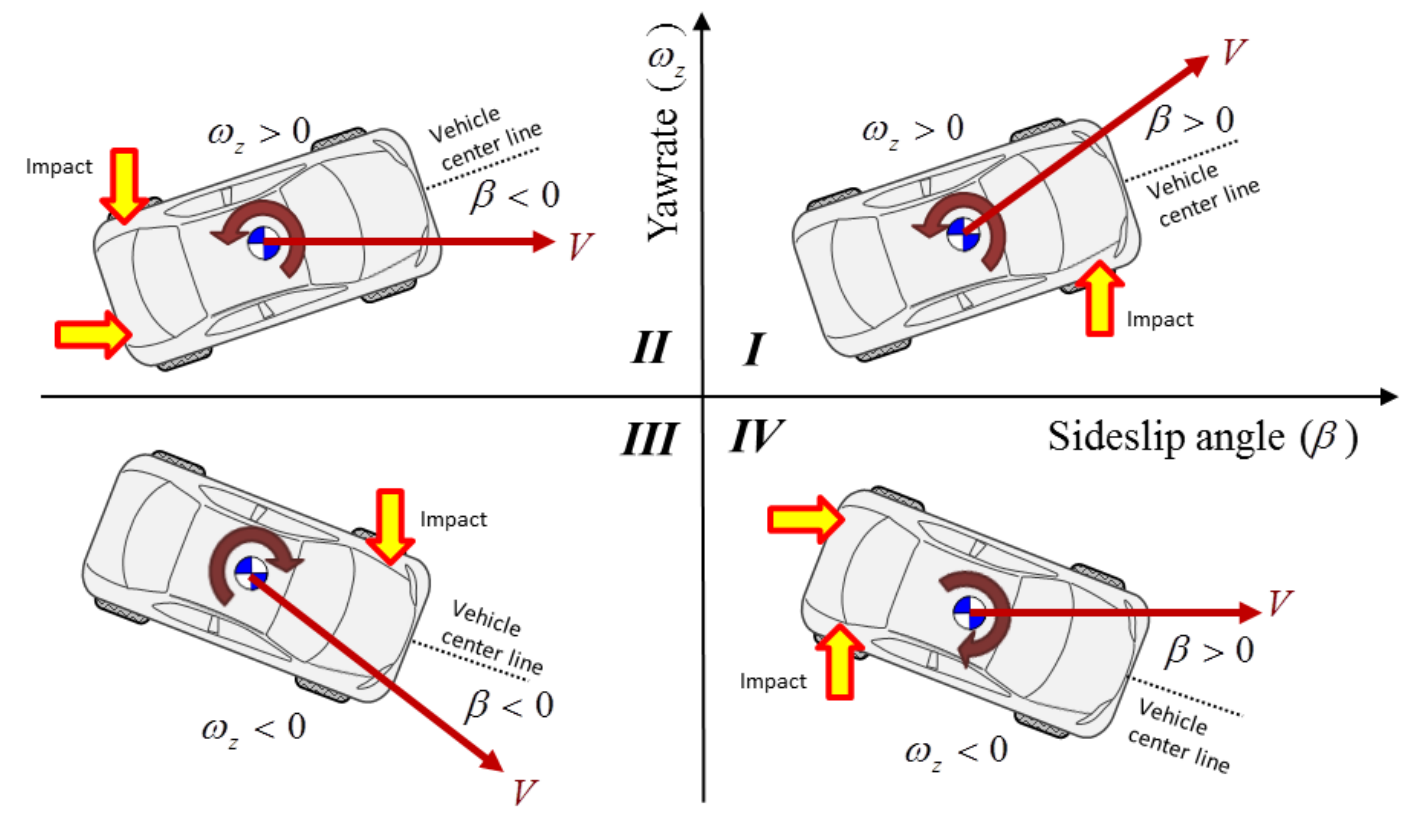

Figure 4.2 A few imagined scenarios with various impact conditions that will create different initial conditions on the phase plane

After an impact, the yaw rate and side slip values can be in any of the four quadrants as Figure 4.2. The vehicle states in quadrants $I$ and III, corresponding to states with yaw rate and side slip with the same sign, tend to move toward the origin. On the other hand, the vehicle states in quadrants $I I$ and $I V$, corresponding to the cases where the signs of yaw rate and side slip are opposite, tend to stay from the origin. An interesting observation, when comparing the length of each state trajectory from the same initial yaw rate, is that the initial sideslip in quadrants $I I$ and $I V$ will take more time to converge toward the origin than the conditions in quadrants $I$ and $I I I$. For example, when looking at the state trajectories from two points where the initial sideslip and yaw rate are $\left(-10^{\circ}, 10^{\circ} / \mathrm{s}\right)$ and $\left(+10^{\circ}, 10^{\circ} \% \mathrm{~s}\right)$ in Figure 4.1, the curve length is quite different. During the time when the curve from the point $\left(+10^{\circ}, 10^{\circ} \%\right)$ converges to the origin, the curve from the point $\left(-10^{\circ}, 10^{\circ} / \mathrm{s}\right)$ does not move much, meaning that larger control efforts 
and longer time are needed for initial conditions in quadrants $I I$ and $I V$ than in quadrants $I$ and $I I I$.

The situation is even worse for states with high initial yaw rates. As shown in Figure 2.12, an external impact to the vehicle can create acceleration higher than $1.0 \mathrm{~g}$ and higher yaw rate than what is possible by steering. Because of the friction limit, there exists a maximum yaw rate that can be generated by steering and the vehicle control becomes harder when the vehicle yaw rate is higher than the maximum steady-state yaw rate, stated in $[67,68]$. The maximum steady-state yaw rate can be approximated by using the relationship in Equations (4.1) with $\dot{\beta}=0$ and by considering limits of handling due to the limited tire forces:

$$
\omega_{z, \max } \approx \frac{\mu \cdot F_{z}}{m \cdot v_{x}}=\frac{\mu \cdot m \cdot g}{m \cdot v_{x}}=\frac{\mu \cdot g}{v_{x}}
$$

Based on this assumption, the maximum steady-state yaw rate $\left(\omega_{z, \max }\right)$ is about $15^{\circ} / \mathrm{s}$, when $v_{x}=30 \mathrm{~m} / \mathrm{s}$. If the initial yaw rate after an impact is higher than $\omega_{z, \max }$, the vehicle states will diverge from the origin on the phase plane initially. The situation is worse in quadrants $I I$ and $I V$ than quadrants $I$ and $I I I$. The maximum steady-state yaw rate is speed dependent and becomes higher as the vehicle speed decreases.

Imagine that there are vehicle states with impact conditions beyond the normal operating range of the conventional ESC. If the side slip angle and yaw rate are larger than what are experienced in typical driving conditions, and ESC tries to control the vehicle back to the origin, it may take a long time to stabilize the vehicle, which could have issues, such as large lateral deviation. It can be seen that it is relatively easy to control the vehicle to the origin for initial states in the convergence region. This is because initial conditions inside the convergence region are moving towards the origin even without control. On the other hand, large control effort is needed when the initial state is located far away from the convergence region. In addition, the control authority is limited when tire slip angles are high. For this reason, in designing an ESC control, the typical range of side slip for vehicle control or parameter estimation is smaller than the location of saddle-node equilibrium along the sideslip angle axis on the phase plane, which indicates a maximum and minimum limit of converging region (typical range is at most \pm 30 degrees [69]). 
Vehicle motions with excessive side slip angle are usually outside of the scope of conventional vehicle stability control systems. For this research, however, it is necessary to use the extended phase plane shown in Figure 4.3. The extended phase plane shows vehicle motion trajectories with a wider range of side slip angles. It should be noted that this phase plane shows multiple equilibria. Even though an initial state (outside of region (1)) may be outside the convergence region of the origin, it can converge to another equilibrium point located at, say, 360 degree side slip angle (region (2)). In addition, it is important to note that fast yaw rate reduction is achieved around multiples of 180 degree slip angles. A detail analysis on this fact will be discussed in the next section. If a control system tries to drive the vehicle with a high initial yaw rate and high side slip angle (e.g., point 'A') back to the origin, the control action will be high. While without any control effort, the vehicle state naturally converges to the alternative equilibrium with a side slip of $360^{\circ}$. In other words, selecting an alternative equilibrium with the final heading angle at multiples of 180 degrees may be beneficial.

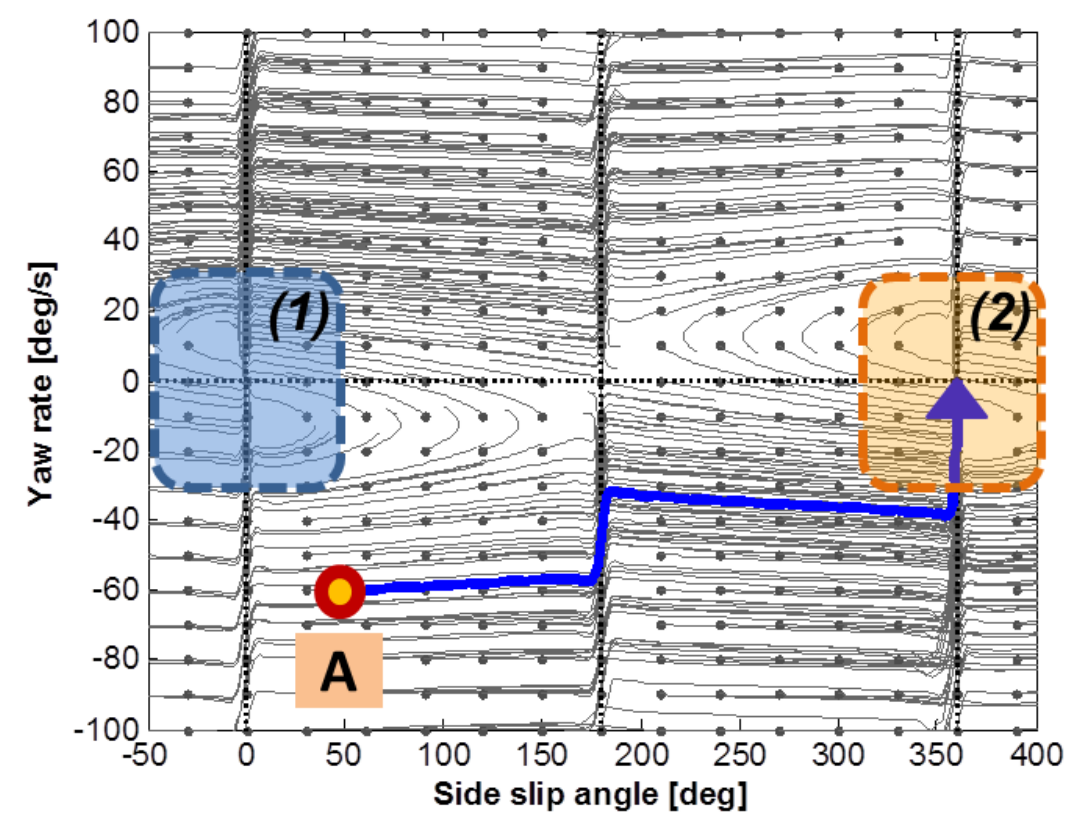

Figure 4.3 Extended phase plane. Region (1) is the convergence region for the origin, while Region (2) represents the one for an alternative equilibrium. Point ' $A$ ' indicates a possible initial state after an impact outside of region (1). Blue line illustrates the resulting vehicle motion without control. 


\subsection{Dynamics Analysis and Beneficial Angles}

Two real world accident scenarios, shown in Figure 4.4 [70] and Figure 4.5 [71], are studied. Both cases have a similar initial condition to point ' $A$ ' in Figure 4.3, then the vehicle in the first case tried to go back to Region (1) while the vehicle in the second case reached another equilibrium point in Region (2).

Figure 4.4 has an initial condition in quadrant $I V$ of Figure 4.2. After the impact, the vehicle's heading angle in the scene is toward the outside of the road. It is shown that the side slip angle of the vehicle keeps growing and the vehicle stops with a severe secondary crash with a roadside pole. According to the analysis in Section 4.1, the induced sideslip from the first collision cannot be easily be diminished and in fact was growing. This is a representative example that shows the difficulty in controlling the vehicle back to the origin from adverse initial conditions.
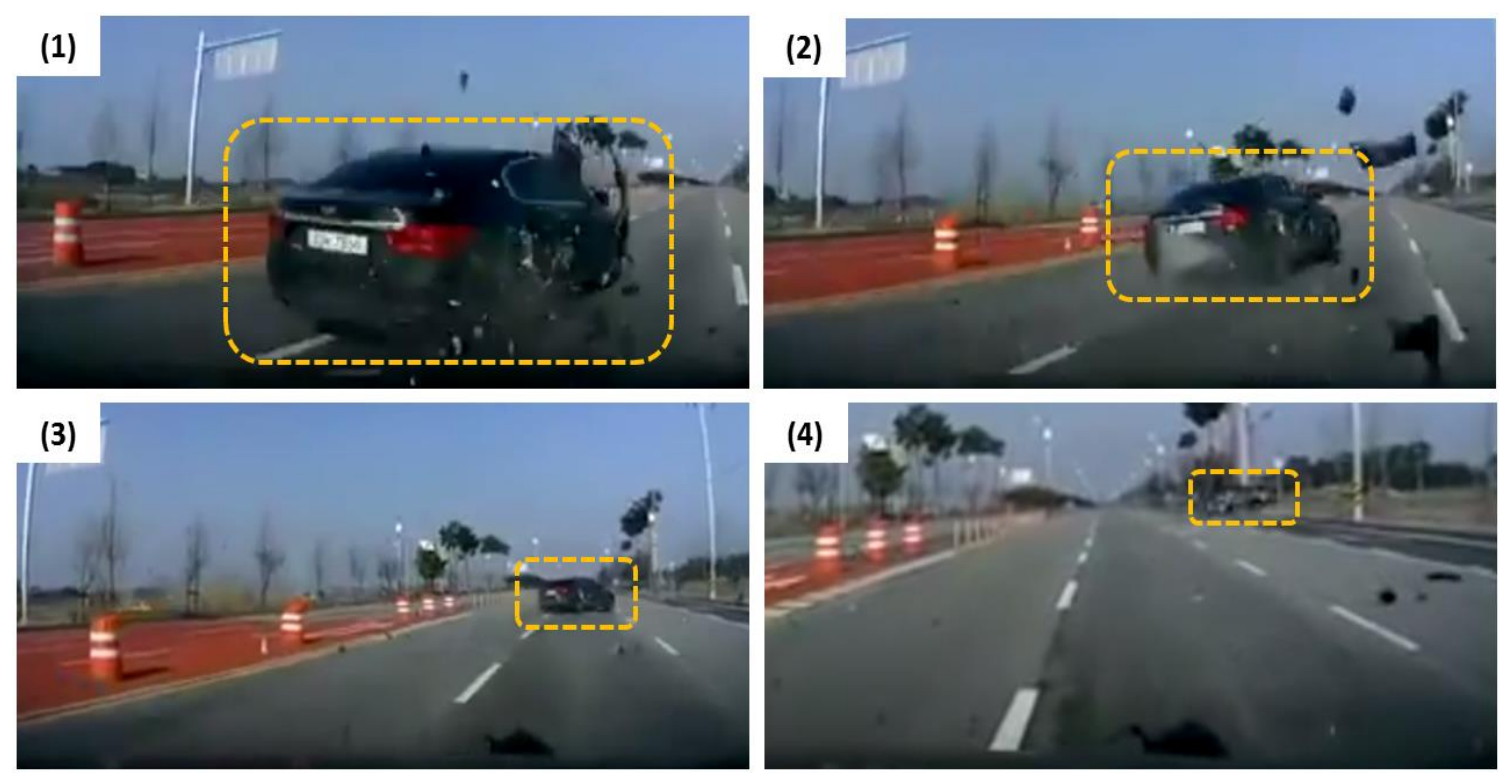

Figure 4.4 A sequential scene captured on dashboard camera in the striking car, which ends with a side pole crash. The sequence is from upper left to lower right [70]

On the other hand, Figure 4.5 can provide insight into the potential benefit of performing extreme vehicle spin motion to $180^{\circ}$ or $360^{\circ}$ (or multiples of $180^{\circ}$ thereafter). Vehicle collisions do not only occur just as accidents, but can happen on purpose. The Precision Immobilization Technique (PIT), shown in Figure 4.5, is a pursuit tactic used 
by the law enforcement to stop a fleeing vehicle. The technique is performed by intentionally steering sharply into the target vehicle to create a large yaw motion that causes the driver in the pursued vehicle to lose control and stop. Figure 4.5 shows a skillful driver in the fleeing vehicle could recover and converge to an alternative equilibrium point. The vehicle motion in Figure 4.5 exemplifies the potential benefit of controlling a vehicle to multiples of $180^{\circ}$ angles.

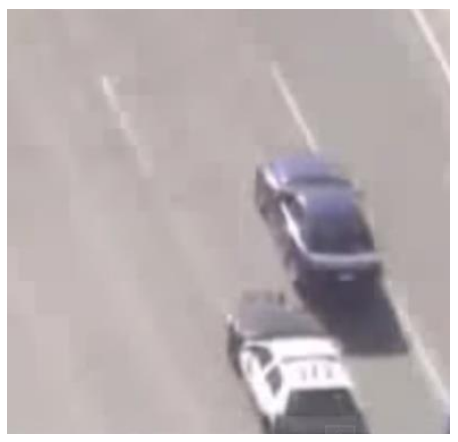

(1) The police car impacts the fleeing car.

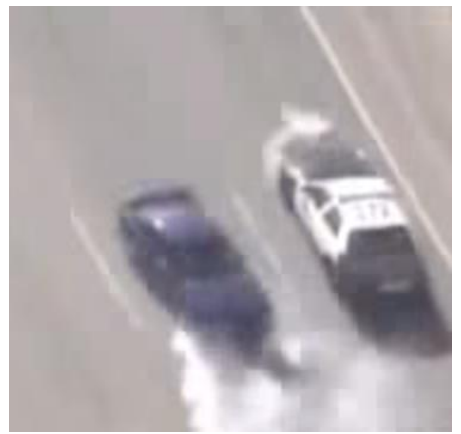

(4) The fleeing car drives in the opposite direction.

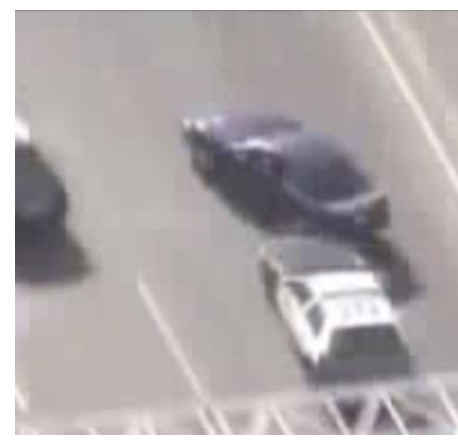

(2) The police car steers sharply and the fleeing car starts to spin.

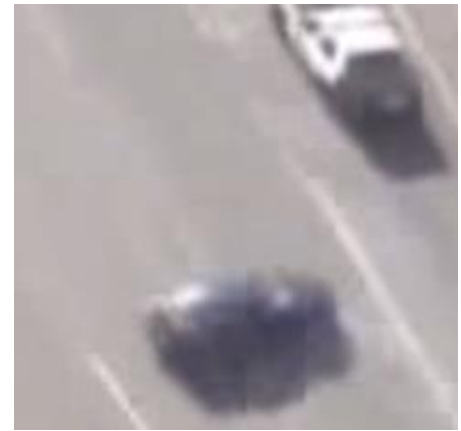

(5) The driver in the fleeing car continues to turn.

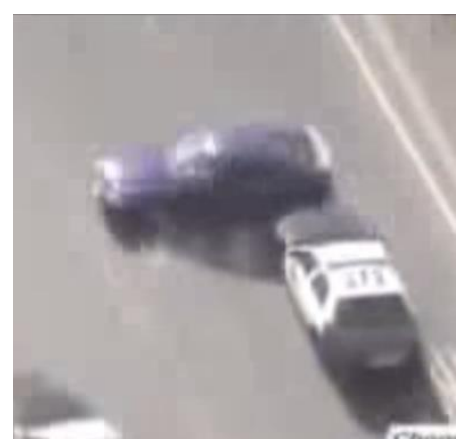

(3) The fleeing car loses control.

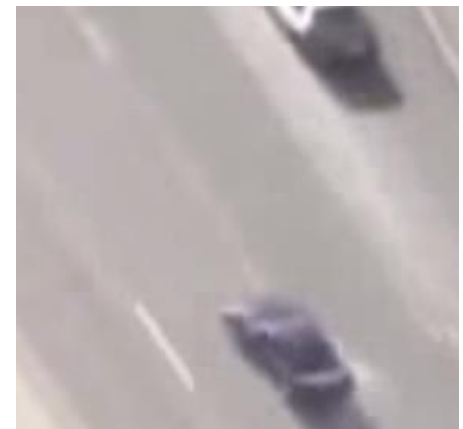

(6) The fleeing car turns $360^{\circ}$ and continues to drive

Figure 4.5 A recovery motion of a fleeing vehicle chased by a law enforcement vehicle executing a PIT (Precision Immobilization Technique) maneuver. The sequence is from upper left to lower right. (Blue color: The suspect vehicle, Black-and-white color: The police vehicle) [71]

To understand the benefit of reaching to a final heading angle at multiple of $180^{\circ}$, the vehicle motion after an external impact is examined by a simulation. The simulation is performed using the commercial software CarSim with the built-in template parameters corresponding to the "Baseline big SUV." It is assumed that the vehicle is initially 
traveling straight with an initial longitudinal speed of $30 \mathrm{~m} / \mathrm{s}(67 \mathrm{mph}, 108 \mathrm{kph})$ and zero initial lateral speed, yaw rate, and roll rate. The collision impact $\left(F_{x_{-} \max }=8.4673 \mathrm{e}+004 \mathrm{~N}\right.$, $F_{y_{-} \max }=-3.9484 \mathrm{e}+004 \mathrm{~N}$ ) lasts for 0.15 seconds and has a sine square force profile, and the impact location is $0.1 \mathrm{~m}$ to the left of the center of the rear bumper. The road is assumed to be flat and straight, and the adhesion is assumed to be homogeneous with the frictional coefficient $\mu=0.7$. The induced yaw moment $\left(M_{\text {Friction }}\right)$ from the tire lateral forces is calculated through the following relationship:

$$
M_{\text {Friction }}=a \cdot\left(F_{y, f_{L}}+F_{y, f_{R}}\right)-b \cdot\left(F_{y, r_{L}}+F_{y, r_{R}}\right)
$$

where, $F_{y, f_{L}}, F_{y, f_{R}}, F_{y, r_{L}}, F_{y, r_{R}}$ are the lateral forces of four tires.

Because driving and braking are not included in the simulation, the tire longitudinal forces are ignored and zero steering angles is assumed. Figure 4.6 illustrates tire forces, tire slip angles, and yaw moments acting on the vehicle.

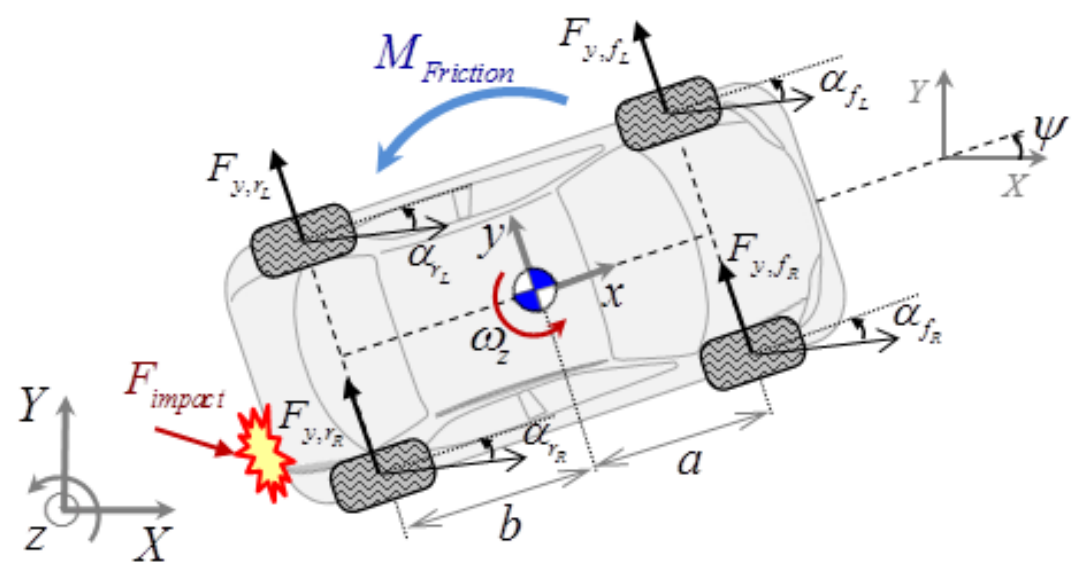

Figure 4.6 Induced yaw moment from the lateral tire forces

Figure 4.7 shows the vehicle motion induced by the crash without control. $F_{y F}$ and $F_{y R}$ denote the sum of the left and right tire forces at each axle. 

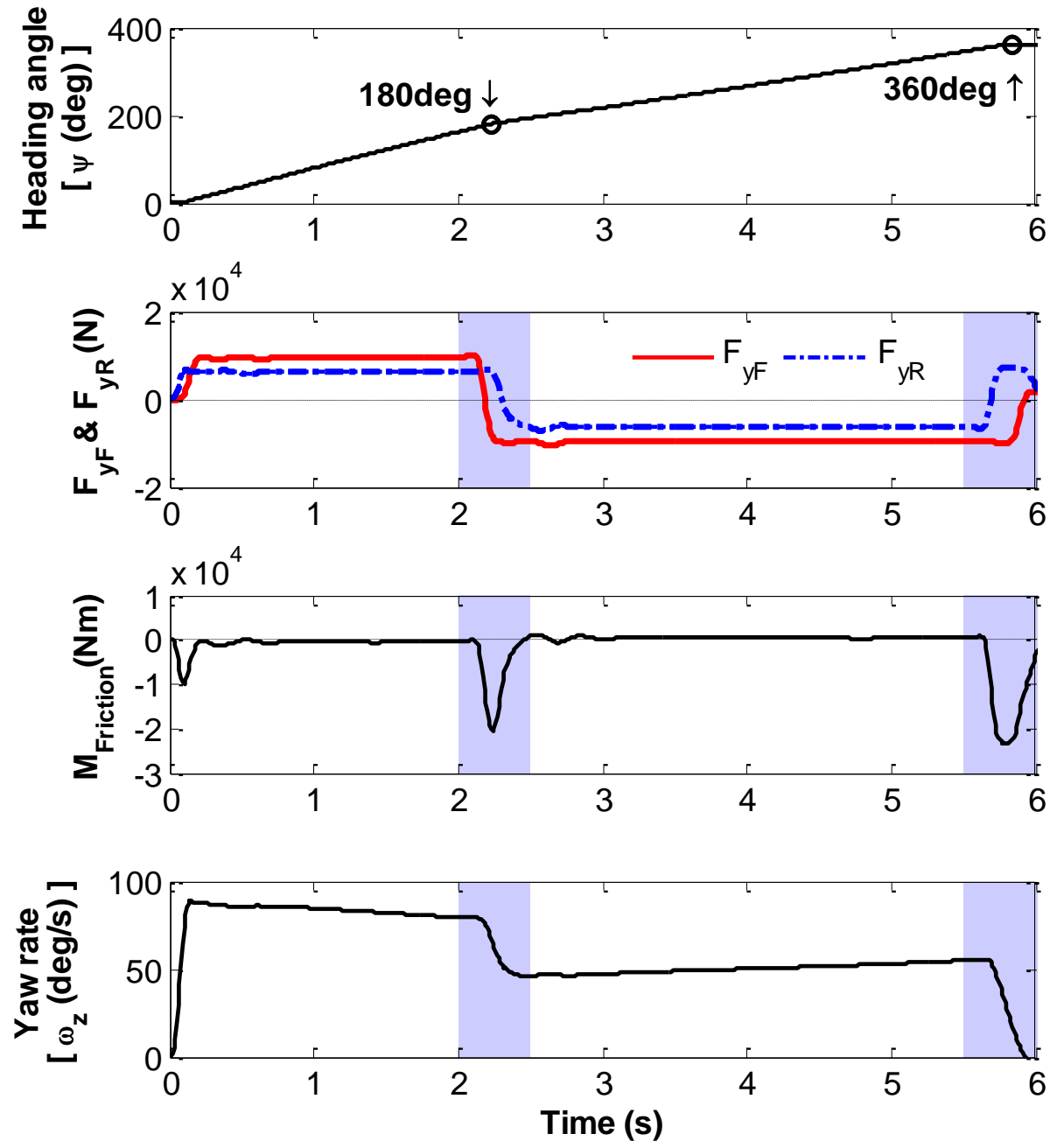

Figure 4.7 Open-loop simulation results showing the vehicle motion, forces, and yaw moment generated by the tires

Interestingly, the induced yaw moment acts as a counter-reaction against the yaw motion and reduces the yaw rate significantly when the vehicle heading angle approaches $180^{\circ}$ and $360^{\circ}$. This situation can be explained by the tire force characteristics shown in Figure 2.7 where the lateral force changes rapidly when the tire slip angle is around $0^{\circ}$ or multiples of $180^{\circ}$ angles, especially when the tires are rolling $(\lambda=0)$. Figure 4.8 shows tire forces change along with tire slip angles. $\alpha_{f}$ and $\alpha_{r}$ denote the average of the left and right tire slip angles at each axle. In the shaded regions, it is shown that the lateral forces of the front and rear tires switch signs at slightly different times. This time difference introduces 
a large induced yaw moment $\left(M_{\text {Friction }}\right)$ when the lateral forces at the two axles are in opposite directions, as illustrated in Figure 4.9. For this reason, significant yaw rate reductions happen.
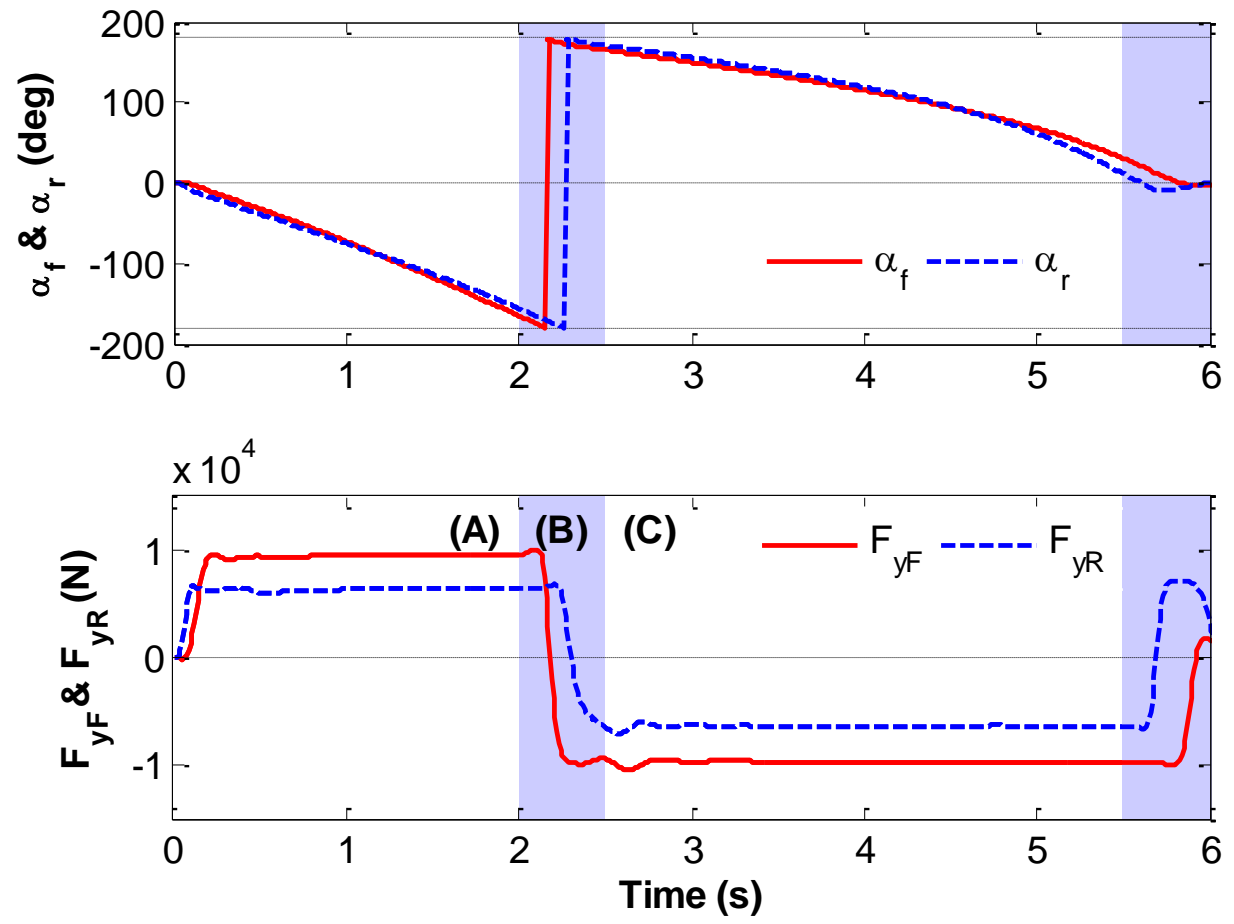

Figure 4.8 Tire lateral forces and slip angle after an impact
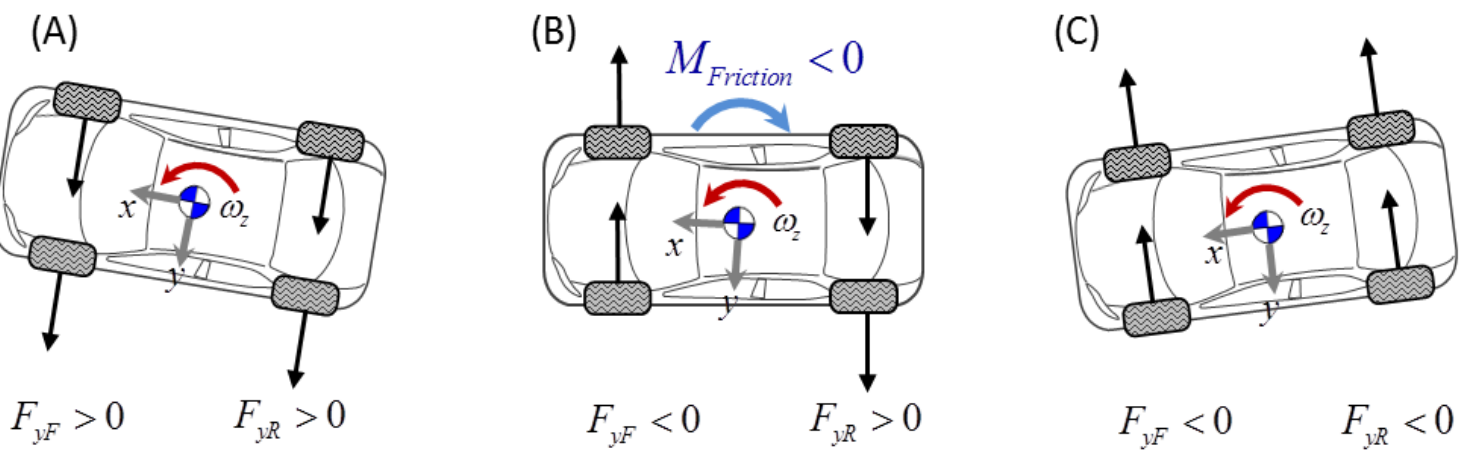

Figure 4.9 Schematic view at (A), (B), and (C) time sequence in Figure 4.8 


\subsection{Vehicle Motion with Open Loop Braking Actions}

Vehicle longitudinal and lateral forces are functions of the slip angles and longitudinal slip ratios of the four tires. In this section, open loop braking actions are applied using Equations (2.7)-(2.11) to compare the transitions of vehicle motions by analyzing the role of individual wheel brakes. First, Figure 4.10 shows example scenarios after several initial impact conditions without any braking. Each circle on the zero heading angle vertical line represents an initial yaw rate after the impact. It is assumed that the vehicle was traveling at a longitudinal speed of $30 \mathrm{~m} / \mathrm{s}(67 \mathrm{mph}, 108 \mathrm{kph})$ with zero heading angles. The gray lines are different initial yaw rates ranging between $10 \% \mathrm{~s}$ and $200 \%$ s. In particular, the red lines in the figures are the trajectories with the following initial conditions: $3 \mathrm{~m} / \mathrm{s}$ lateral speed and $100 \% \mathrm{~s}$ yaw rate. The horizontal black dashed lines represent traffic lanes on the road and the space between adjacent lines is $3.65 \mathrm{~m}$. When the vehicle position on the red line crosses road lines, those events are marked with the '*' symbols.
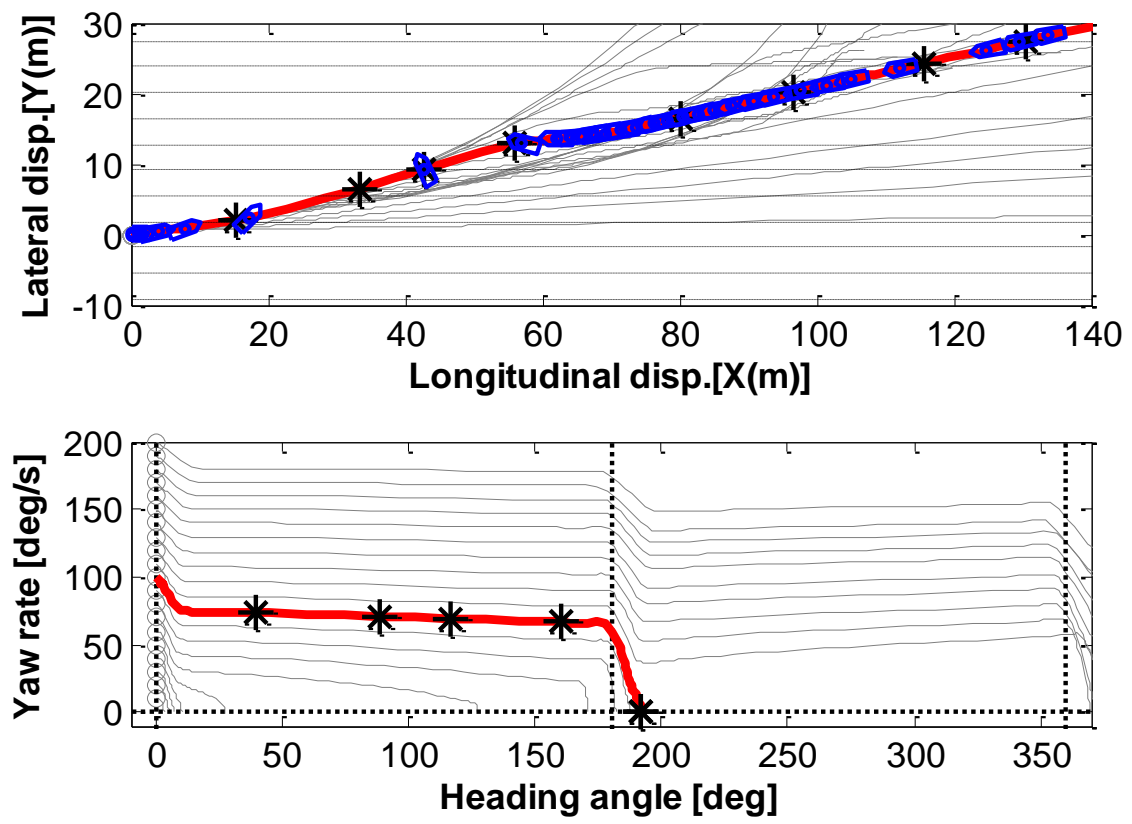

Figure 4.10 Projected vehicle position and motion without any control (*: indicates lane boundary crossing)

This result shows that the final heading angles vary depending on the initial yaw rate. And the lateral deviation gets larger as the vehicle trajectory advances because the 
vehicle converges to an arbitrary heading angle. However, a significant yaw rate reduction occurs when the vehicle heading angle approaches $180^{\circ}$.

Next, Figure 4.11 shows the effects of different braking control actions in the phase plane. The red lines in the plots highlight the trajectories with $100 \%$ s yaw rate initial condition for comparison. Since the tire forces are affected significantly by the tire slip ratios and slip angles, the vehicle yaw rate and heading angle are quite different for the three braking actions.

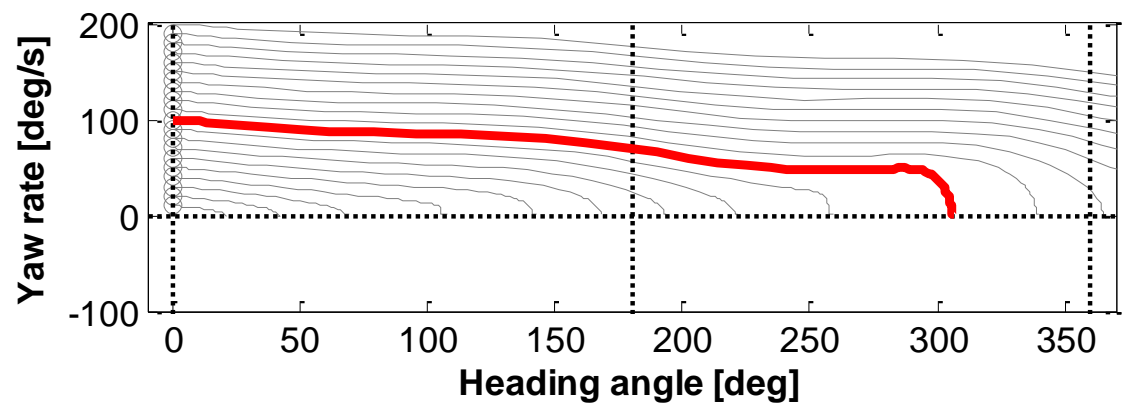

(a)

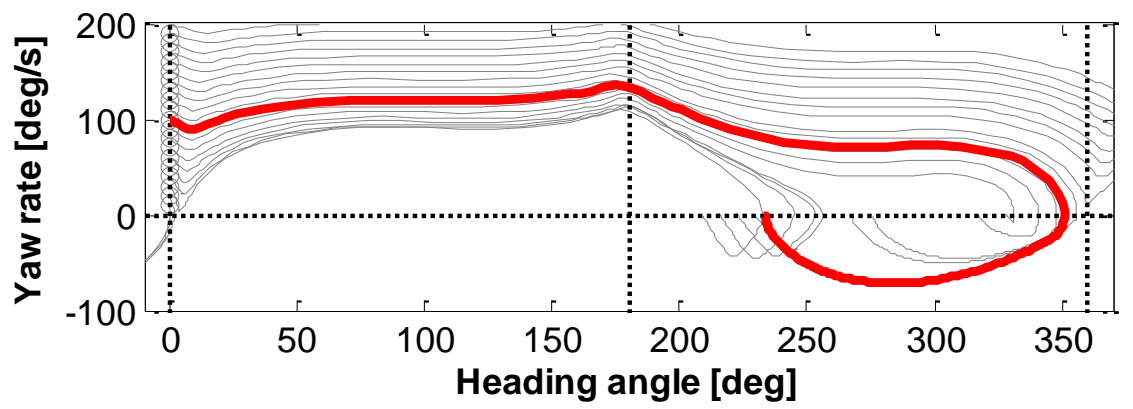

(b)

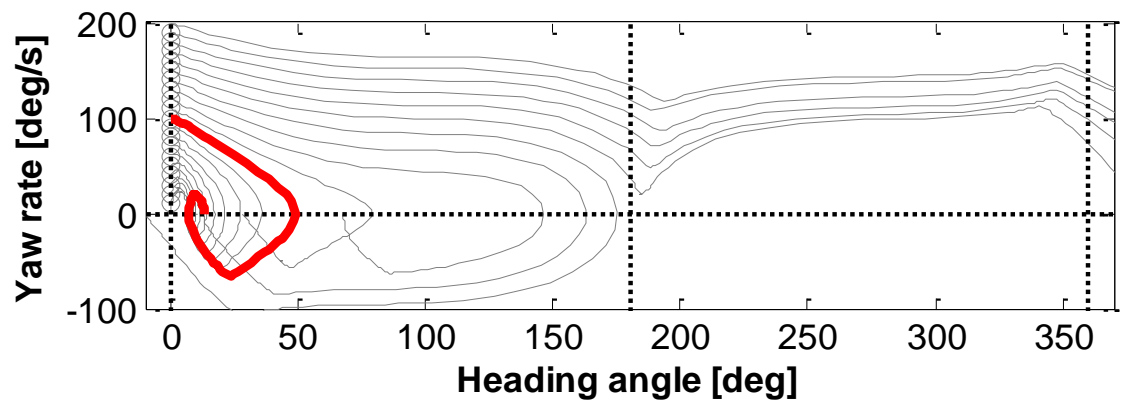

(c)

Figure 4.11 Comparison of braking actions with the initial state $v_{x}=30 \mathrm{~m} / \mathrm{s}$ and $\omega_{z}=100 \% \mathrm{~s}$. (a) 4-wheel brake lock. (b) Rear 2-wheel brakes lock. (c) Front 2-wheel brake lock.

As opposed to the results in (b) and (c), applying full braking to all four wheels in (a) generates smooth yaw rate trajectories. Because of the tire coupling nonlinearities, tire 
lateral force is reduced when longitudinal braking is applied and ideally lateral tire force profile becomes similar to the sine wave when the wheel is locked $(\lambda=-1)$, as shown in Figure 4.12. In other words, when signs of the tire lateral forces change (slip angles are around $0^{\circ}$ and $\pm 180^{\circ}$ ), the slope of lateral tire force in wheel braking case is lower than the free rolling case $(\lambda=0)$. Therefore, no significant yaw rate reduction occurs in Figure 4.11 (a).

Meanwhile, different transitions of yaw rate are shown in locking the rear 2-wheel and front 2-wheel cases because the distances from the CG to the front/rear wheel axles are different. As illustrated in Figure 4.12 and Figure 4.13, lateral tire forces are reduced when braking is applied. In particular, the direction of variations of rear tire lateral forces $\left(\Delta F_{y, r_{L}}\right.$ and $\left.\Delta F_{y, r_{R}}\right)$, defined in the Equation (4.8), are in the same direction with the yaw rate induced by the impact.

$$
\Delta F_{y, i}=F_{y, i}^{\text {brake }}-F_{y, i}^{\text {nobrake }} \quad\left(i \in f_{L,} f_{R,}, r_{L}, r_{R}\right)
$$

For this reason, the yaw rate response in Figure 4.11 (b) is higher than the response in Figure 4.10. On the other hand, the variations of the front tire lateral forces $\left(\Delta F_{y, f_{L}}\right.$ and $\Delta F_{y, f_{R}}$ ) act against the direction of the initial yaw rate, in turn the yaw rate is reduced when braking is applied.

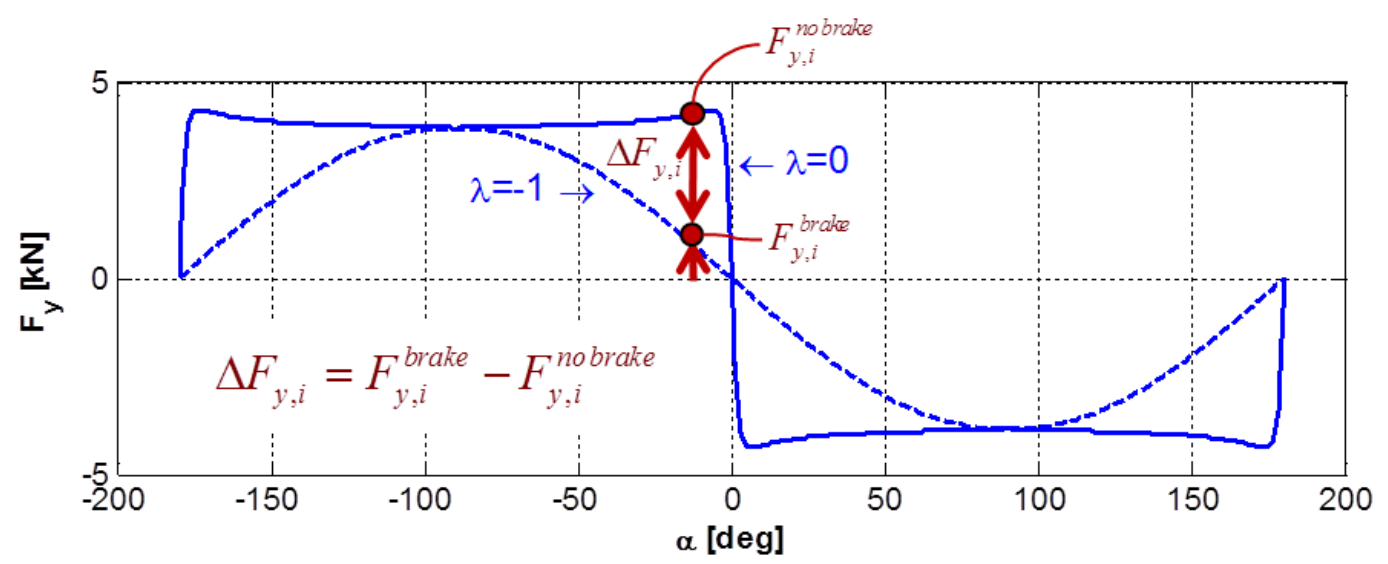

Figure 4.12 Reduction in tire lateral force when braking is applied $\left(i \in f_{L}, f_{R}, r_{L}, r_{R}\right)$ 


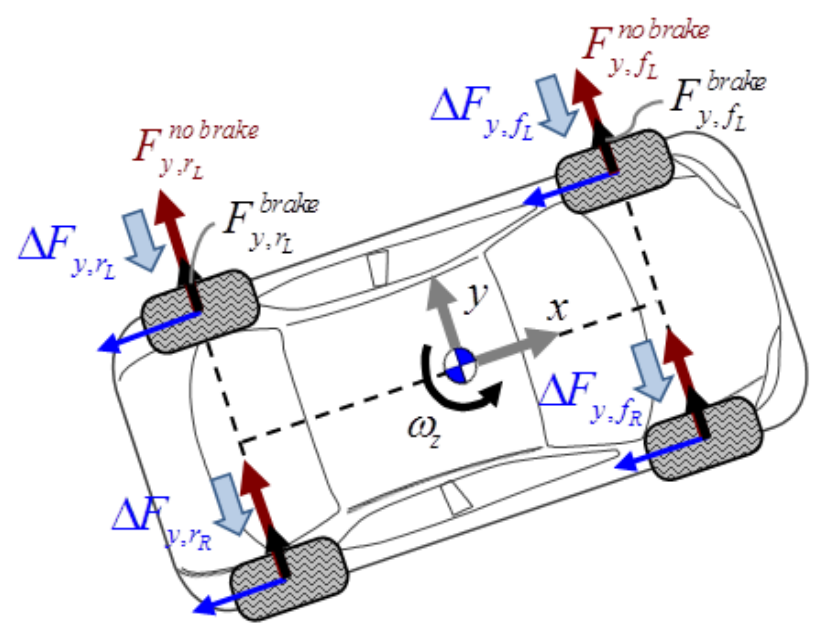

Figure 4.13 Effects of vehicle yaw moment when the lateral tire forces are affected by braking

\subsection{Optimized Trajectory for Secondary Collision Safety}

Minimizing the lateral deviation from the original path and aligning the vehicle heading angle to the original course direction are both important to reduce the threat of secondary collisions. Lateral deviation is a hazard that may cause a collision with vehicles in other lanes or stationary objects on the roadside. Heading angle is crucial because broadsided by another vehicle can cause severe occupant injuries due to the fact that vehicles have less crash energy absorbing materials on the side than on the front or the back of the vehicle.

An optimization problem is solved to obtain proper control actions. The objective function takes into account both lateral course deviation and vehicle heading angle. To solve the optimization problem, a gradient descent approach is implemented to find the optimal control signals to balance between minimum lateral deviation and safe heading angle. The 'fmincon' function in the MATLAB toolbox is used to solve this constrained optimization problem [72]. The control inputs (longitudinal slip ratios of the four tires) are found to minimize the objective function under the constraints: 


$$
\begin{aligned}
& \min _{\lambda_{f L}, \lambda_{f R}, \lambda_{r L}, \lambda_{r R}} J\left(\lambda_{f L}, \lambda_{f R}, \lambda_{r L}, \lambda_{r R}\right) \\
& \text { subject to } \quad-1 \leq \lambda_{f L}, \lambda_{f R}, \lambda_{r L}, \lambda_{r R} \leq 0
\end{aligned}
$$

where, $\lambda_{f L}, \lambda_{f R}, \lambda_{r L}, \lambda_{r R}$ are slip ratios of the front axle tires and rear axle tires, respectively.

The objective function to be optimized is:

$$
J=w_{1} \cdot \sum_{i=1}^{N}(Y(i))^{2}+w_{2} \cdot \sum_{i=1}^{N}(2 \pi-\psi(i))^{2}
$$

The objective function $J$ consists of two terms. The first term penalizes lateral displacement from the original path and the second term penalizes the vehicle heading angles to $2 \pi$, in this particular simulation. Effects of each term can be evaluated by varying the weighting factors, $w_{1}$ and $w_{2}$.

Figure 4.14 compares four cases: (1) without control, (2) control to minimize lateral displacement, (3) control heading angle to $2 \pi$, and (4) control both heading angle and lateral displacement. Each graph shows the resulting vehicle trajectories and slip ratios from the optimization. The initial vehicle states are the same as the one in Figure 4.10. Optimization results from the displacement control in Figure 4.14 (b) show a small lateral deviation. Although the vehicle converges to a small but non-zero heading angle, it comes back to the original lane. On the other hand, the vehicle in Figure 4.14 (c) turns to $2 \pi$ heading angle, but a large lateral deviation from the original lane is introduced while achieving the final heading. Lastly, the vehicle trajectory in Figure 4.14 (d) has both small lateral deviation and the final heading angle. 


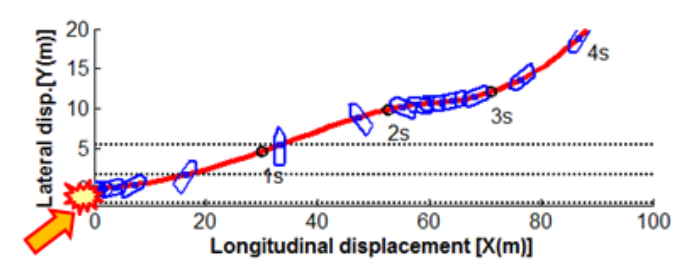

(a) No control action
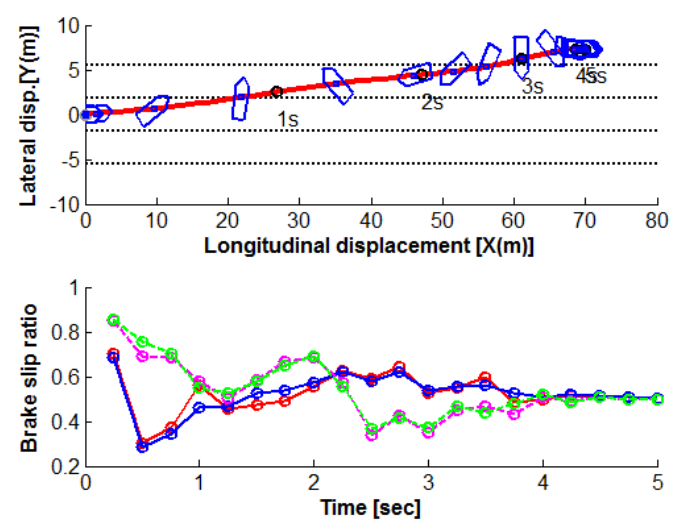

(c) Heading angle control $\left(w_{1}=0, w_{2}=1\right)$
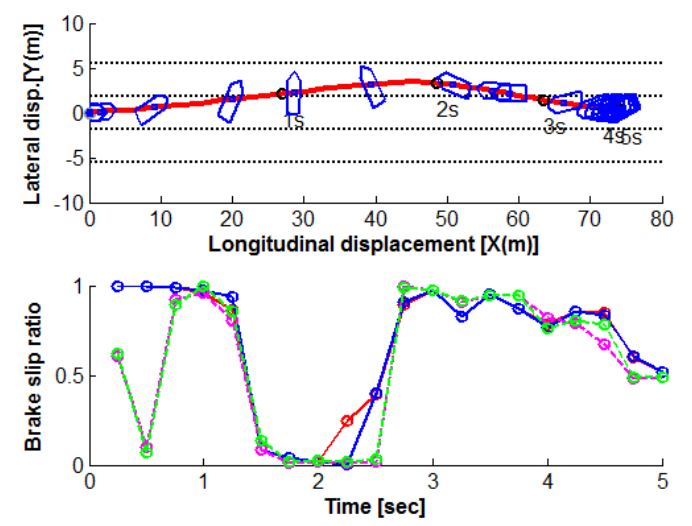

(b) Displacement control $\left(w_{1}=1, w_{2}=0\right)$
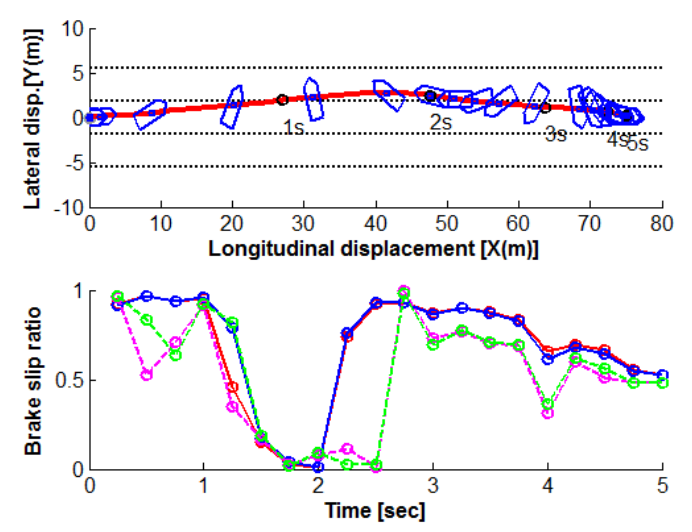

(d) Consider both displacement and heading angle $\left(w_{1}=1, w_{2}=1\right)$

Figure 4.14 Vehicle trajectories with different objective functions

Based on the result in Figure 4.14, the following objective function is designed:

$$
J=w \cdot \sum_{i=1}^{N}\left(Y_{s}(i)\right)^{2}+\sum_{i=1}^{N}\left(\left|\psi_{\text {mod }}(i)-\frac{\pi}{2}\right|-\frac{\pi}{2}\right)^{2}
$$

where $Y_{s}$ is the lateral displacement from the lane center in the Earth-fixed coordinate system, $\psi_{\text {mod }}$ is the modulo operation of heading angles with $\pi\left(\psi_{\bmod }=\bmod (\psi, \pi)\right)$, which computes the remainder that results from performing a division with $\pi$. A weighting factor $w$ is used to balance the effects of the two terms in the cost function. It should be noted that the desired heading angle in the objective function varies depending on the 
current heading angle state. Since the output range of the term, $\bmod (\psi, \pi)$, is $[0, \pi]$ even when the heading angle $(\psi)$ is greater than $\pi$, the errors with $\pi,(\pi-\bmod (\psi, \pi))$, decrease as slip angle approaches multiples of $180^{\circ}$, as shown in Figure 4.15.

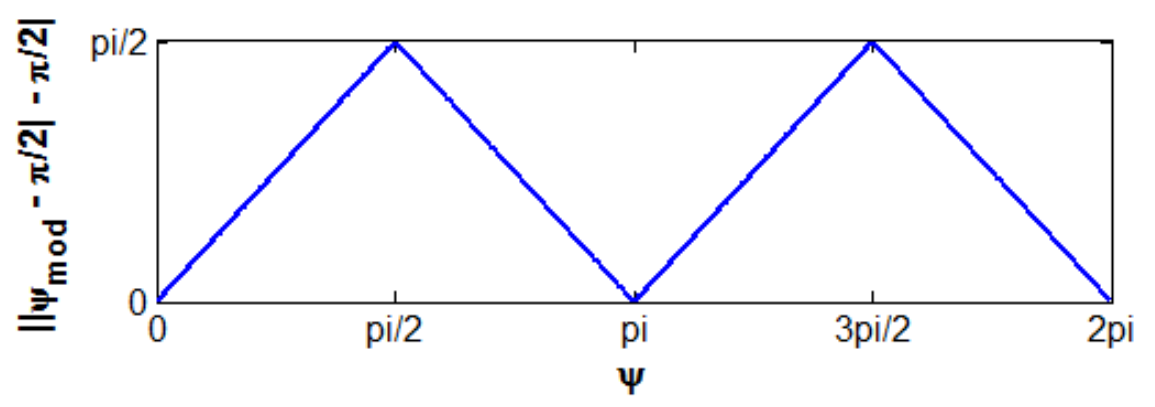

Figure 4.15 Penalty function for the heading angle

The second component of the cost function is designed to control the vehicle heading angle to multiples of $180^{\circ}$. To avoid the situation where the vehicle's longitudinal axis is perpendicular to the road coordinates, the highest penalty value will be assigned to when the heading angle is close to $90^{\circ}$ and $270^{\circ}$. Then, as the objective function gets minimized, the lateral deviations decrease and the heading angles approach multiples of $180^{\circ}$, at the same time. A "trade-off" between small lateral deviations and safe heading angles can be made by adjusting the weighting factor in the objective function.

Figure 4.16 shows the wheel brake actions from the optimization scheme. In this simulation, all measurements such as position, speed, yaw rate, and heading angle are assumed to be available and accurate, and actuator delays are ignored. Figure 4.16 (a) and (b) compares the trade-offs between lateral displacement and heading angle with different weighting factors. The result in (a) achieves $180^{\circ}$ heading angle with one lane offset from the course, while the control in case (b) bring the vehicle to the original lane with a $360^{\circ}$ turn. If the vehicle is free from secondary collisions in spinning to $360^{\circ}$, the result in (b) will be preferred. On the contrary, if there is a risk of secondary collision during spinning, keeping the final angle at $180^{\circ}$ may be safer. 

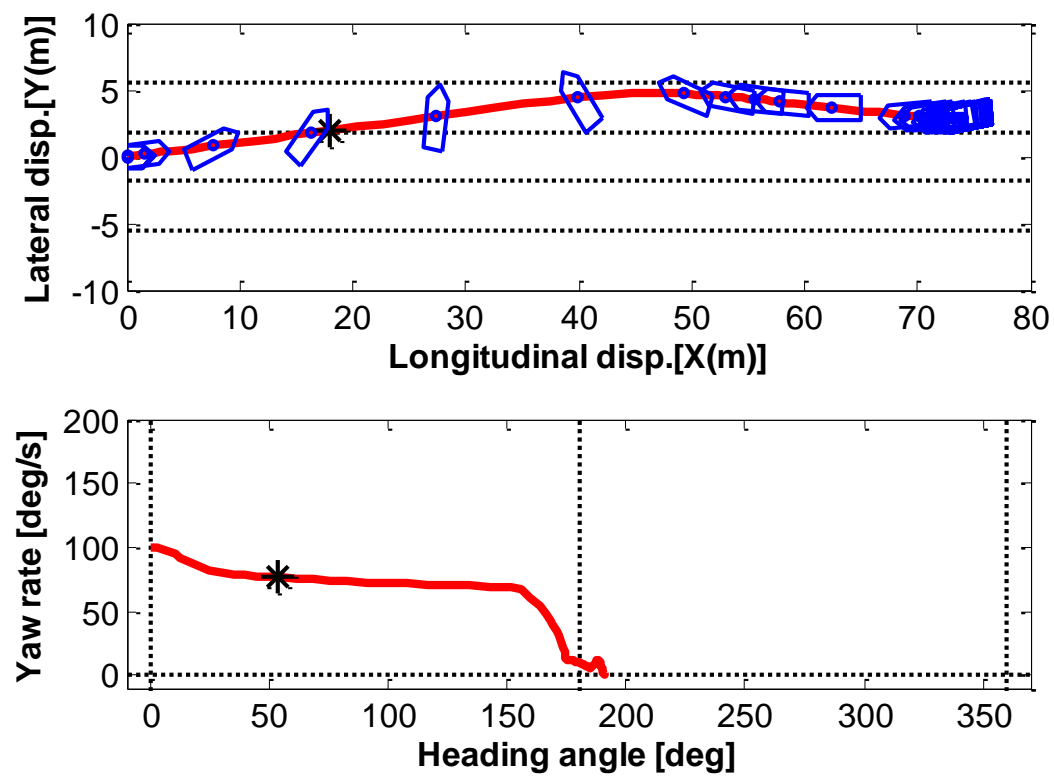

(a) $\mathrm{w}=10$
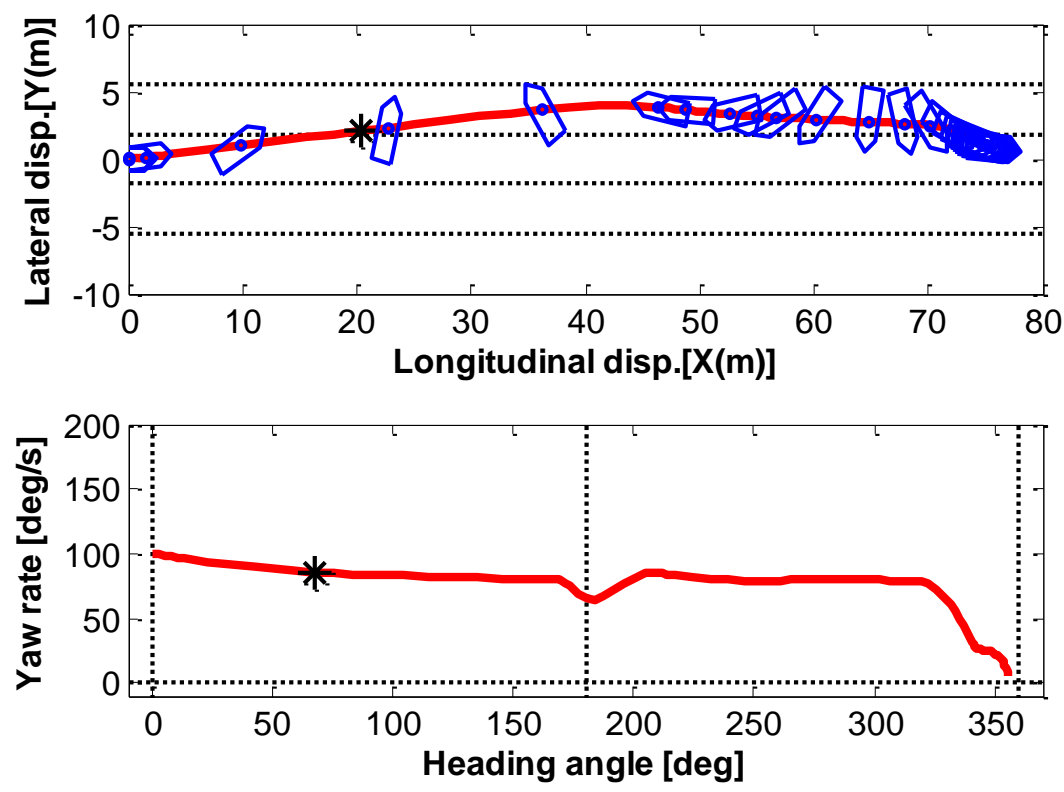

(b) $\mathrm{w}=1000$

Figure 4.16 Optimized vehicle trajectory for two different weightings 


\subsection{Desired Final Heading Angles}

Vehicle control will be performed based on the control target determined by a desired vehicle state. Based on the predicted vehicle motion from collision force estimation, the desired final heading angle is found. Since we found that a heading angle at multiples of $180^{\circ}$ is beneficial to avoid broadside impact, the final heading angle is a major parameter for the desired vehicle state. By changing the initial conditions of the vehicle states, the desired final heading angles are obtained by solving the optimization problem in Equation (4.9). This optimization is conducted offline and the results can be implemented as a lookup table. Figure 4.17 shows example points included in the lookup table. The initial conditions for longitudinal and lateral velocities are: $V_{X}=30 \mathrm{~m} / \mathrm{s}, V_{Y}=2$ $\mathrm{m} / \mathrm{s}$, and the yaw rate conditions $\left(\omega_{z}\right)$ vary from $30 \%$ s to $170 \% \mathrm{~s}$. It is noted that the final heading angle is higher as the initial yaw rate becomes larger.

More extensive simulations are performed with various initial conditions shown in Figure 4.18, and the results are used to setup the lookup table for the desired final heading angle. Given two different initial longitudinal speeds, Figure 4.18 shows the desired final heading angle levels. Although similar patterns are shown in (a) and (b), it is clear that the heading angle decreases as vehicle speed increases. The results also indicate that the initial lateral speed $\left(V_{Y}\right)$ has less influence on the desired heading angle than the initial yaw rate $\left(\omega_{z}\right)$.

In summary, this section demonstrates the process of determining the desired heading angle for vehicle motion control. The vehicle motion after an impact is reviewed through the phase plane analysis, and it is demonstrated that the multiples of $180^{\circ}$ angle is beneficial in maintaining small lateral displacement. The process in this section is the intermediate step between collision strength prediction and vehicle motion control. The results from the motion prediction in the previous chapter are used as initial conditions of the vehicle motion to determine the final heading angle, and then the control algorithm in the next chapter is performed based on the lookup table information determined by the offline optimization solutions in this chapter. 


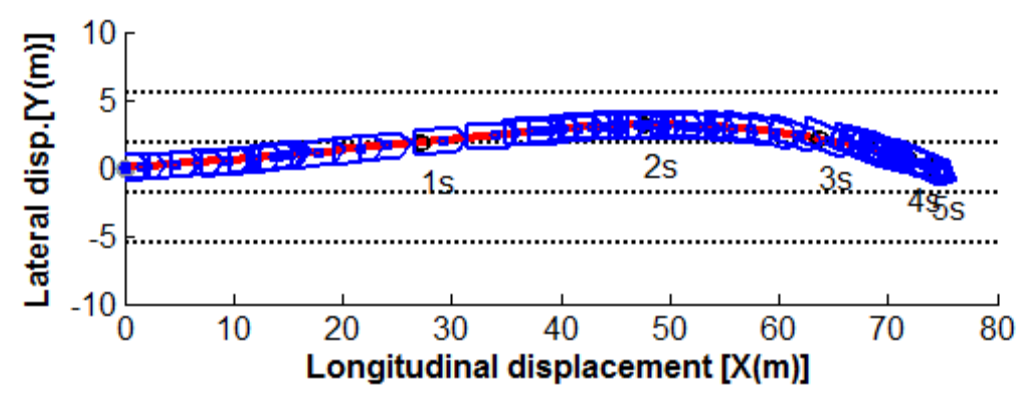

(a) Desired final heading angle: $0^{\circ}$, when initial yaw rate $\omega_{z}=30^{\circ} / \mathrm{sec}$

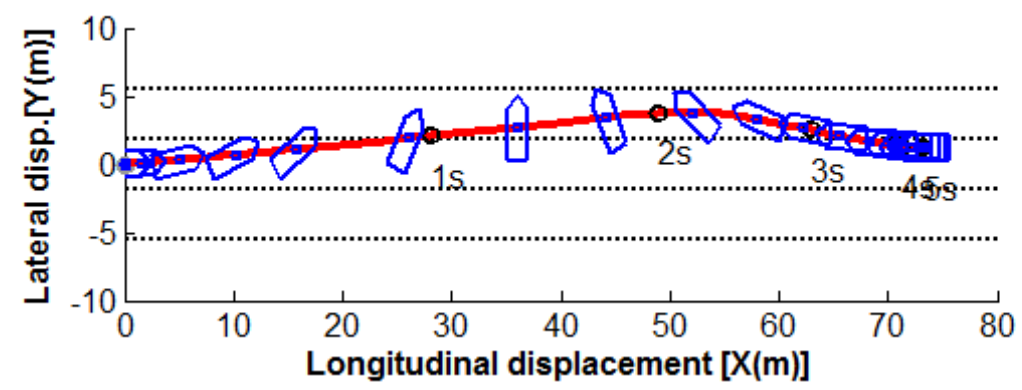

(b) Desired final heading angle: $180^{\circ}$, when initial yaw rate $\omega_{z}=70^{\circ} / \mathrm{sec}$

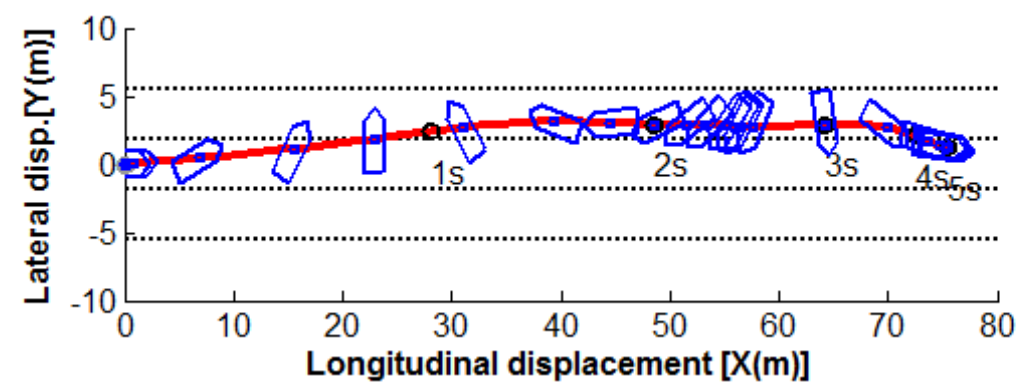

(c) Desired final heading angle: $360^{\circ}$, when initial yaw rate $\omega_{z}=120^{\circ} / \mathrm{sec}$

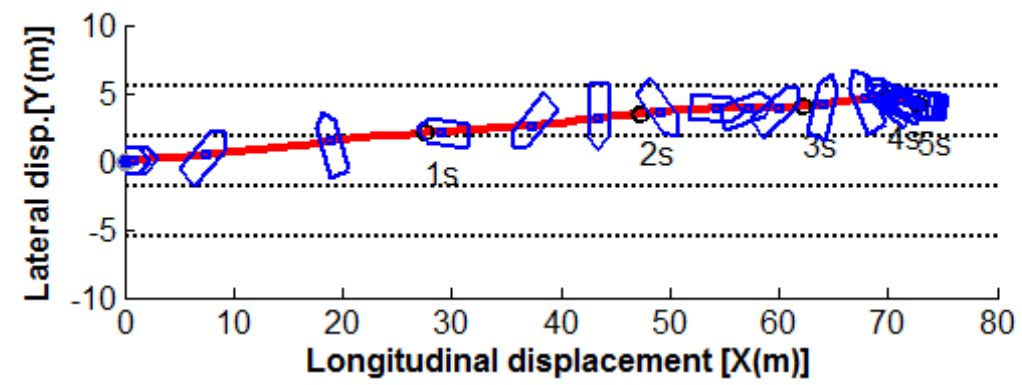

(d) Desired final heading angle: $540^{\circ}$, when initial yaw rate $\omega_{z}=170^{\circ} / \mathrm{sec}$

Figure 4.17 Optimization results showing desired final heading angles for various initial yaw rates (Initial conditions: $V_{X}=30 \mathrm{~m} / \mathrm{s}, V_{Y}=2 \mathrm{~m} / \mathrm{s}$ ) 


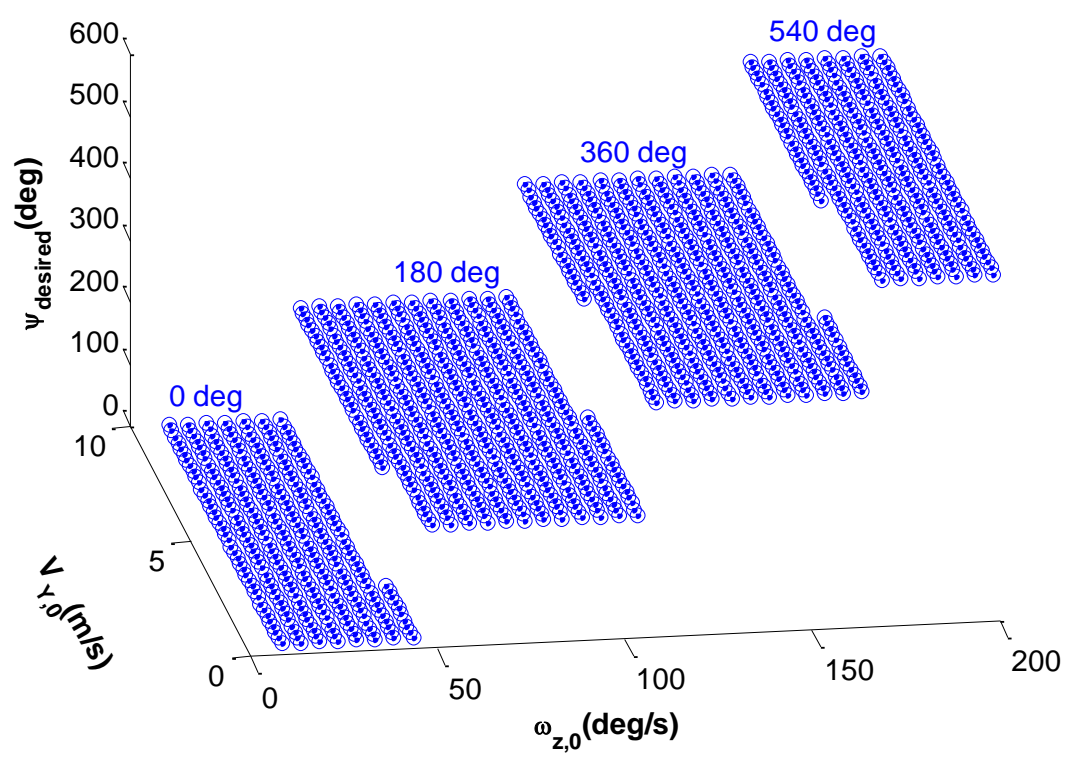

(a) $V_{X}=30 \mathrm{~m} / \mathrm{s}$

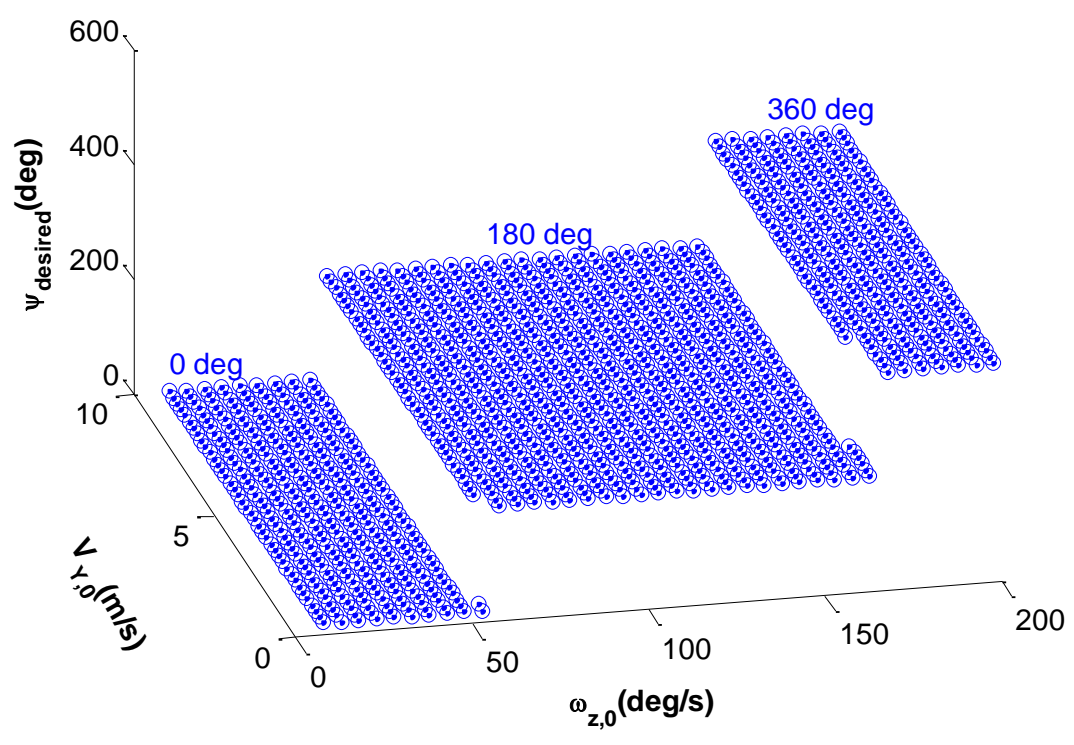

(b) $V_{X}=20 \mathrm{~m} / \mathrm{s}$

Figure 4.18 Desired final heading angle maps depending on vehicle speed 


\section{CHAPTER 5 \\ CONCLUSION AND FUTURE WORK}

In this chapter the design of a post-impact control system based on differential braking is proposed and verified. The main objective of the control is to minimize the lateral deviation and bring the vehicle heading angle to a desired angle which is parallel to the road traveling direction. The development of the controller will be based on the 3DOF planar vehicle model, and the controller is assumed to have access to all necessary vehicle states.

\subsection{Controller Design Concept}

The proposed control design structure is illustrated in Figure 5.1. The control system is composed of two parts, upper level controller and lower level controller. The upper level controller determines required virtual forces and moment acting on the vehicle CG, which are the longitudinal and lateral forces and the yaw moment.
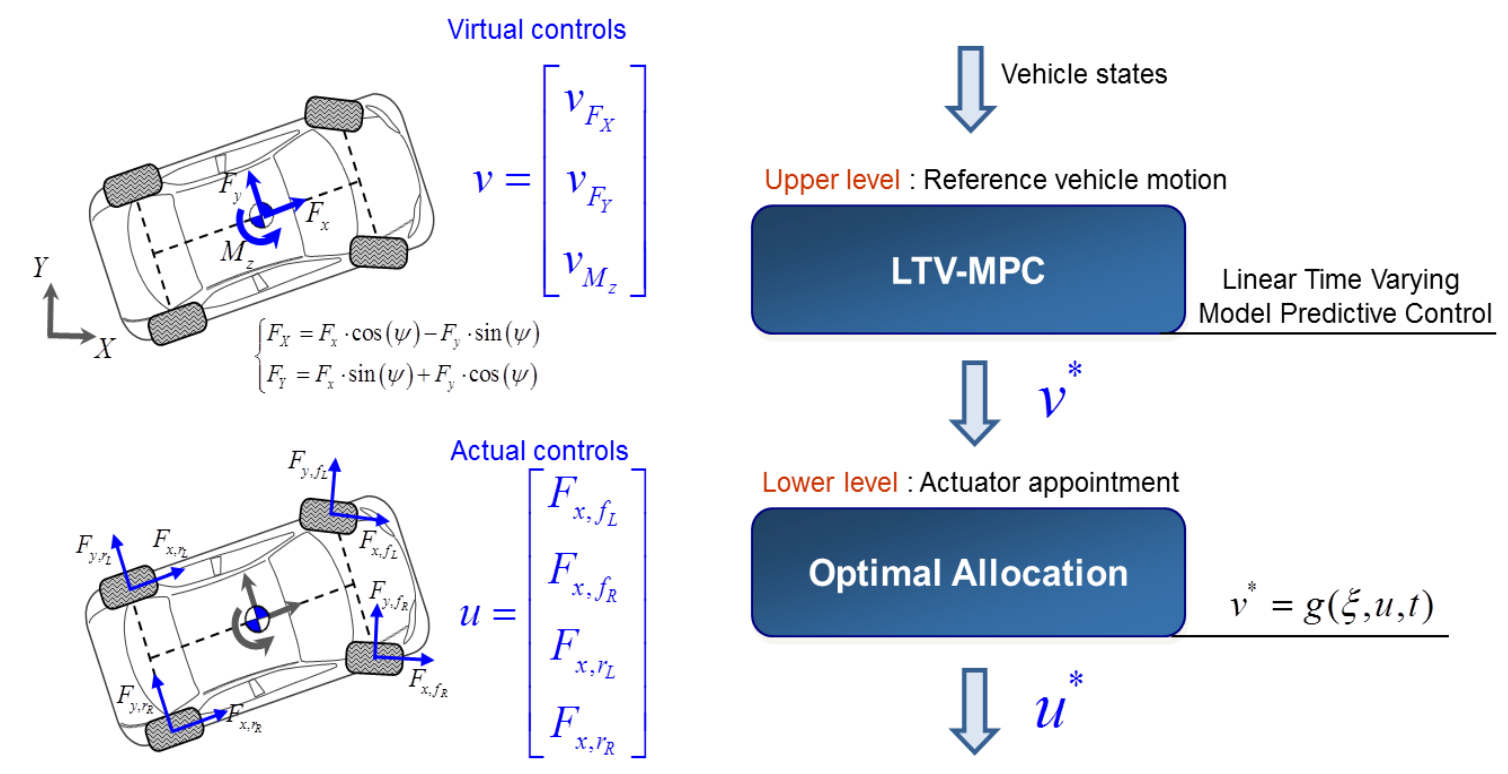

Figure 5.1 Hierarchical frame work for the vehicle control systems 
The Model Predictive Control (MPC) scheme is selected because it intrinsically handles constraints and multivariable problems. Specifically, the linear-time varying MPC design methodology is applied to handle the vehicle nonlinearities. The purpose of the lower level control is to determine the actual wheel braking controls based on the virtual forces and yaw moments from the upper level.

\subsection{Model Predictive Control}

The Model Predictive Control (MPC) methodology has been used in many automotive applications [73, 74] to achieve fuel economy, emissions, and safety [75]. In the MPC scheme, the plant models are used to predict the response of the dynamical system. MPC uses the predictions and current measurements to determine optimal control inputs that minimize the cost function over a prediction horizon, while satisfying the state and input constraints. As mentioned in [76], MPC has a few benefits: First, it naturally handles multi-input-multi-output control problems. Second, it incorporates physical constraints which reflect control actuator and output state limits in a structured way. Third, it uses the predicted model behaviors in the prediction horizon. Fourth, tuning parameters can be well understood and it is easy to tune.

In this research, three important criteria were considered in the design of the MPC controller: (1) the controller should handle the highly nonlinear vehicle motion, (2) the tire authority constraints should be considered, and (3) the computational load should be within acceptable range. To deal with vehicle nonlinearity, the nonlinear MPC (NMPC) strategy can be a choice. However, its computation load is too high and the solver is not yet reliable enough, which were known issues for NMPC in real-time implementation [77]. For vehicle safety systems, reliable real-time performance while operating at the limits of handling capability is required [78]. To overcome these issues, the idea of using a time-varying linear model was proposed in [79]. In that study, the plant models are linearized and the tire cornering stiffness is identified at each time step. Although they limited the operation within the linear region, their algorithm was evaluated with a test vehicle. Adopting the scheme shown in [79], we use the same linear time-varying MPC 
(LTV-MPC) scheme in this thesis but do not restrict the operational region to the linear region.

\subsection{Equations for LTV-MPC Controller Design}

The vehicle control model is derived from the planar nonlinear vehicle dynamics introduced in Chapter 2.1.2. Considering the fact that the longitudinal braking forces on each tire are independently controlled, Equations (2.7)-(2.11) can be used to construct a six-state nonlinear state space form:

$$
\dot{\xi}(t)=f(\xi, t)+g(\xi, u, t)
$$

where, $\xi=\left[\begin{array}{llllll}X & V_{X} & Y & V_{Y} & \psi & \omega_{z}\end{array}\right]$ and

$$
\begin{aligned}
& f(\xi, t)=\left[\begin{array}{c}
V_{X} \\
-\left\{F_{y, f_{L+R}}^{0} \cdot \sin (\psi+\delta)+F_{y, r_{L+R}}^{0} \cdot \sin (\psi)\right\} / m \\
V_{Y} \\
\left\{F_{y, f_{L+R}}^{0} \cdot \cos (\psi+\delta)+F_{y, r_{L+R}}^{0} \cdot \cos (\psi)\right\} / m \\
\omega_{z} \\
\left\{\begin{array}{c}
a \cdot F_{y, f_{L+R}}^{0} \cdot \cos (\delta)-b \cdot F_{y, r_{L+R}}^{0} \\
-\frac{T_{W}}{2} \cdot\left(F_{y, f_{R}}^{0}-F_{y, f_{L}}^{0}\right) \cdot \sin (\delta)
\end{array}\right\} / I_{z z}
\end{array}\right] \\
& g(\xi, u, t)=\left[\begin{array}{c}
0 \\
\left\{F_{x, f_{L+R}} \cdot \cos (\psi+\delta)+F_{x, r_{L+R}} \cdot \cos (\psi)\right\} / m \\
0 \\
\left\{F_{x, f_{L+R}} \cdot \sin (\psi+\delta)+F_{x, r_{L+R}} \cdot \sin (\psi)\right\} / m \\
0 \\
\left\{\begin{array}{l}
a \cdot F_{x, f_{L+R}} \cdot \sin (\delta)+\frac{T_{W}}{2} \cdot\left(F_{x, r_{R}}-F_{x, r_{L}}\right) \\
+\frac{T_{W}}{2} \cdot\left(F_{x, f_{R}}-F_{x, f_{L}}\right) \cdot \cos (\delta)
\end{array}\right\} / \mathrm{I}_{z z}
\end{array}\right]
\end{aligned}
$$




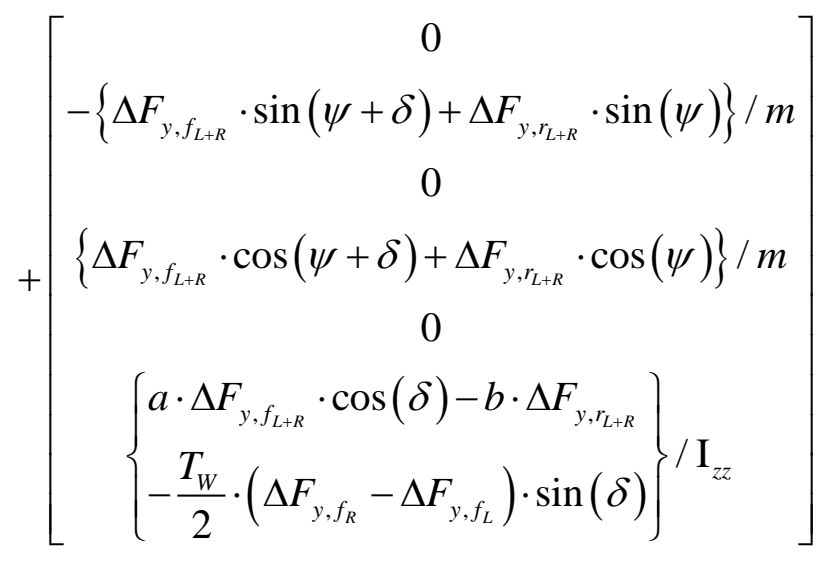

Here, $u$ represents the independent 4-wheel brake control actions ( $u=$ $\left[\begin{array}{llll}F_{x, f_{L}} & F_{x, f_{R}} & F_{x, r_{L}} & F_{x, r_{R}}\end{array}\right], \mathrm{F}_{\mathrm{y}, \star}^{0}$ are the lateral tire forces at a known slip angle under zero longitudinal slip conditions (no braking), and $\Delta F_{y, \oplus_{\ominus}}$ are the induced lateral tire force differences $\left(\Delta F_{y, \oplus_{\ominus}}=F_{y, \oplus_{\ominus}}-F_{y, \oplus_{\ominus}}^{0}\right)$ caused by the combined-slip effect under braking. It should be noted that $f(\xi, t)$ is not related to the actuator control inputs $(u)$, while $g(\xi, u, t)$ is split into two parts: the direct effect of control inputs $\left(F_{x, \oplus_{\ominus}}\right)$, and the indirect effect (disturbance) due to lateral force variance $\left(\Delta F_{y, \oplus_{\ominus}}\right)$. Later, we will solve the control problem in two steps: first, we will solve the virtual control input $g(\xi, u, t)$, and then we will compute the tire force $F_{x, \oplus_{\ominus}}$ that will track the virtual control input, despite the combined-slip tire nonlinearities.

The control structure is separated into two parts: LTV-MPC and optimal allocation algorithm [80]. These two optimization problems exploit the feasible control bounds based on physical constraints so that the resulting control signals are achievable by the tires. To determine the control constraints based on the vehicle states, coupling between the tire longitudinal and lateral forces and actuation limits are considered. The overall control structure is shown in Figure 5.2. At each time step, equations of motion in the state space form are linearized around the operating point acquired from the last time step. After discretizing the linearized state space equation, the optimization equations are constructed with a cost function and constraints. The optimization problem is solved by the quadratic programming routine, and its solution is fed to the optimal allocator to find the physical brake control actions. 


\section{Equations of motion and state space form}

Using estimated or measured vehicle states, nonlinear dynamic properties are linearized at every predefined operating time steps

$$
\begin{gathered}
\dot{\xi}=f(\xi, \mathrm{u}, t) \\
\dot{\xi}=A(t) \cdot \xi+B(t) \cdot v
\end{gathered}
$$

$u$ : Real actuator controls $v$ : virtual controls

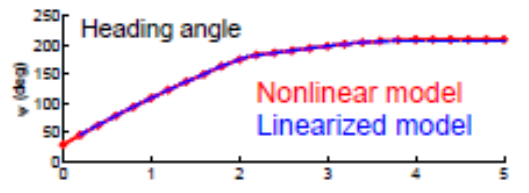

\section{Receding horizon optimal control with constraints}

Cost function for optimization:

$$
J_{k}=\sum_{i=1}^{N+1} \tilde{\xi}_{k+i}^{T} Q \tilde{\xi}_{k+i}+\sum_{i=0}^{N} v_{k+i}^{T} R v_{k+i}
$$

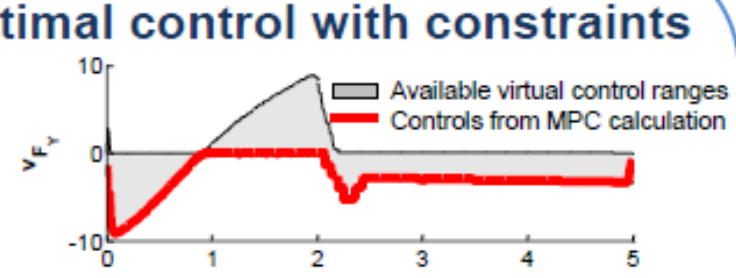

Control objective formulation:

$$
\begin{aligned}
& \left\{\begin{array}{l}
\left\{\begin{array}{l}
\min _{\rightarrow-N-1} J_{k} \\
\text { subject to } h(\underset{\rightarrow N}{\Xi}, \underset{\rightarrow N-1}{V})<0
\end{array}\right. \\
\underset{\rightarrow \rightarrow N-1}{V}=\left[v_{k}, v_{k+1}, \cdots, v_{k+N-1}\right] \\
\underset{\rightarrow N}{\Xi}=\left[\xi_{k+1}, \xi_{k+2}, \cdots, \xi_{k+N}\right]
\end{array}\right. \\
& \left\{\begin{array}{l}
\left\{\begin{array}{l}
\min _{\rightarrow-N-1} J_{k} \\
\text { subject to } h(\underset{\rightarrow N}{\Xi}, \underset{\rightarrow N-1}{V})<0
\end{array}\right. \\
\underset{\rightarrow \rightarrow N-1}{V}=\left[v_{k}, v_{k+1}, \cdots, v_{k+N-1}\right] \\
\underset{\rightarrow N}{\Xi}=\left[\xi_{k+1}, \xi_{k+2}, \cdots, \xi_{k+N}\right]
\end{array}\right. \\
& \left\{\begin{array}{l}
\left\{\begin{array}{l}
\min _{\rightarrow-N-1} J_{k} \\
\text { subject to } h(\underset{\rightarrow N}{\Xi}, \underset{\rightarrow N-1}{V})<0
\end{array}\right. \\
\underset{\rightarrow \rightarrow N-1}{V}=\left[v_{k}, v_{k+1}, \cdots, v_{k+N-1}\right] \\
\underset{\rightarrow N}{\Xi}=\left[\xi_{k+1}, \xi_{k+2}, \cdots, \xi_{k+N}\right]
\end{array}\right. \\
& \left\{\begin{array}{l}
\left\{\begin{array}{l}
\min _{\rightarrow-N-1} J_{k} \\
\text { subject to } h(\underset{\rightarrow N}{\Xi}, \underset{\rightarrow N-1}{V})<0
\end{array}\right. \\
\underset{\rightarrow \rightarrow N-1}{V}=\left[v_{k}, v_{k+1}, \cdots, v_{k+N-1}\right] \\
\underset{\rightarrow N}{\Xi}=\left[\xi_{k+1}, \xi_{k+2}, \cdots, \xi_{k+N}\right]
\end{array}\right.
\end{aligned}
$$
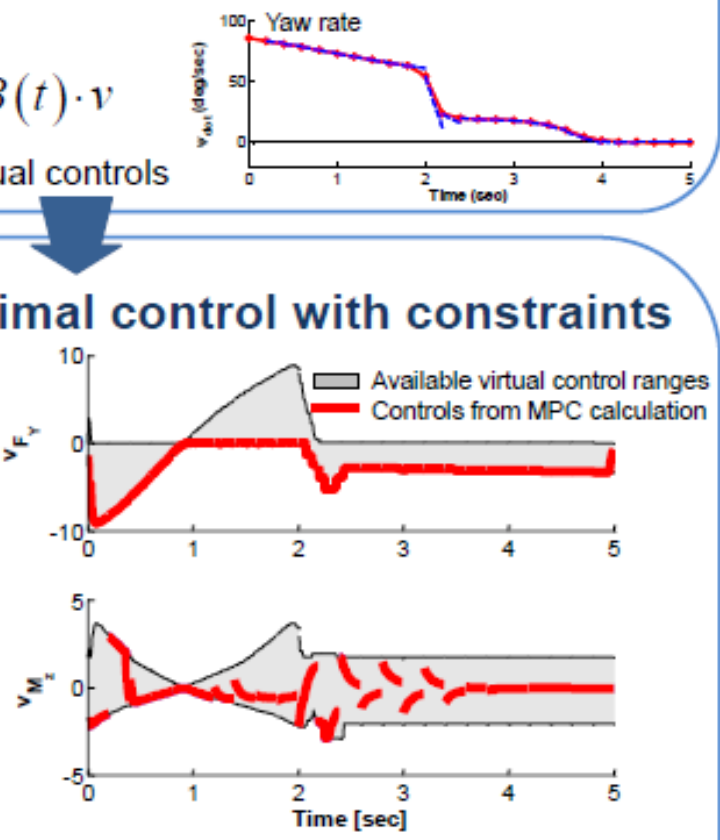

Constraints considered with the coupling effect between the tire longitudinal and lateral forces

\section{Optimal allocation}

Determine the effective physical brake control actions using the nonlinear relationship between the virtual controls $\left(v_{k}^{*}\right)$ and the actual controls $\left(u_{k}\right)$ :

$$
v_{k}^{*}=g\left(\xi_{k}, u_{k}\right) \quad\left(v^{*} \in R^{2}, u^{*} \in R^{4}\right)
$$

Allocation objective formulation:

$$
\begin{aligned}
& \min _{u_{k}}\left(\left\|u_{k}\right\|_{2}^{2}+w \cdot\left\|\eta_{k}\left(v_{k}^{*}, v_{k-1}^{*}, u_{k-1}\right)-G_{k-1} \cdot u_{k}\right\|_{2}^{2}\right) \\
& \text { subject to } \underline{u}\left(\xi_{k}\right) \leq u_{k} \leq \bar{u}\left(\xi_{k}\right)
\end{aligned}
$$$$
\left.G_{k-1} \triangleq \frac{\partial g}{\partial u}\right|_{\hat{s}_{k-1}, u_{k-1}}
$$

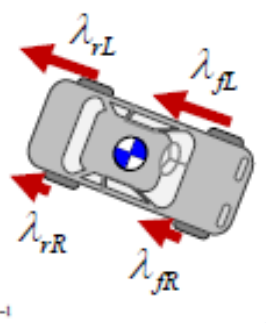

Figure 5.2 Flow chart of the control methodology 


\subsection{Linear Time Varying Model Predictive Control (LTV-MPC)}

\subsubsection{Overview}

The first step to implement the LTV-MPC is the linearization of the nonlinear vehicle dynamics at the current vehicle state. The linearization continues at every linearizing time step, and a quadratic programming structure is constructed based on the linearized formulation $[76,79]$. This method decomposes the nonlinear design problem into several linear sub-problems. The successive linearization points do not need to be equilibriums. From these points, MPC finds a control sequence which minimizes a linear quadratic cost function over the prediction horizon.

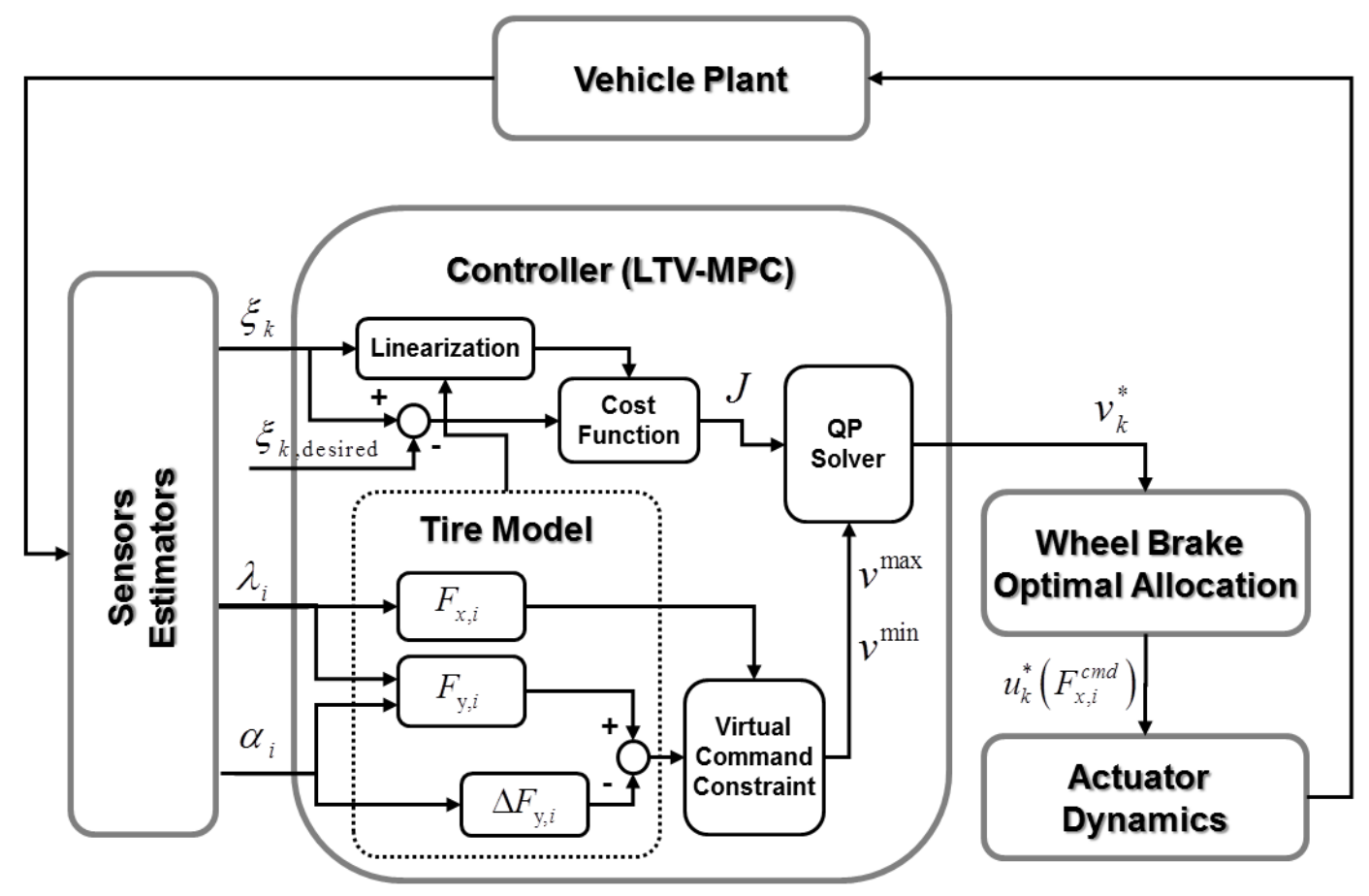

Figure 5.3 Architecture of the proposed control system

The overall control structure is shown in Figure 5.3. The desired vehicle states are first compared with their current states. Then, in response to the state errors, the LTVMPC controller determines the desired virtual controls based on the QP (Quadratic Programming) optimization solution under feasible vehicle dynamic constraints. Next, the optimal control allocation process maps the virtual control demand onto individual 
wheel brake forces. In the last stage, actuator controllers manipulate physical variables, such as wheel cylinder braking pressures, to achieve the desired tire forces. Then, the actuator actions affect the vehicle motion and the resulting vehicle states are measured or estimated for feedback control.

\subsubsection{Linearizing at Non-Equilibrium Points}

Let the second term in Equation (5.1) be the virtual control input (v):

$$
g(\xi, u, t)=B_{v} \cdot v
$$

Considering the nonlinear dynamics shown in Equation (5.1), the linearization is done through Taylor expansion around points $\left(\xi_{0}\right)$ :

$$
\dot{\xi}(t) \approx f\left(\xi_{0}, t\right)+A(t) \cdot\left(\xi-\xi_{0}\right)+B_{v} \cdot v(t)
$$

where,

$$
A(t)=\left.\frac{\partial f(\xi, t)}{\partial \xi}\right|_{\xi_{0}}, \quad B_{v}=\left[\begin{array}{llll}
0 & 1 & 0 & 0 \\
0 & 0 & 0 & 1
\end{array}\right]^{T}, \quad v(t)=\left[\begin{array}{c}
v_{F_{Y}} \\
v_{M z}
\end{array}\right]
$$

Rearrange the equation as:

$$
\dot{\xi}(t)=A(t) \cdot \xi+B_{v} \cdot v(t)+\mathrm{B}_{\tau} \cdot \tau(t)
$$

where,

$$
\begin{gathered}
B_{\tau}=I_{4} \quad(=4 \times 4 \text { identity matrix }) \\
\tau(t)=f\left(\xi_{0}, t\right)-A(t) \cdot \xi_{0}
\end{gathered}
$$

Here, we exclude the first two states $\left(X, V_{X}\right)$ and the model is reduced to a 4-state system $\left(Y, V_{Y}, \psi, \omega_{z}\right)$ because we are mainly concerned with the vehicle lateral displacement error and yaw directional motion. From Equation (5.6), the numerical discretization is applied to build a linear time-varying, discrete time, state-space system with a sampling time $\left(T_{s}\right)$ : 


$$
\xi_{k+1}=A_{d, k} \cdot \xi_{k}+B_{v, d} \cdot v_{k}+B_{\tau, d} \cdot \tau_{k}
$$

Here, we assume that the coefficients in the system and input matrices are constant over the time horizon $n$ :

$$
\begin{aligned}
& \bar{A} \triangleq A_{d, k} \approx A_{d, k+1} \approx \cdots \approx A_{d, k+n-1} \\
& \bar{B}_{v} \triangleq B_{v, d} \\
& \bar{B}_{\tau} \triangleq B_{\tau, d}
\end{aligned}
$$

And, $\tau_{k}$ is also assumed to be constant over the horizon $n\left(\bar{\tau}:=\tau_{k} \approx \tau_{k+1} \approx \cdots \approx\right.$ $\left.\tau_{k+n-1}\right)$. The sequence of prediction state over the time horizon ( $n$ steps) can be expanded as:

$$
\begin{aligned}
\xi_{k+1}= & \bar{A} \xi_{k}+\bar{B}_{v} v_{k}+\bar{B}_{\tau} \bar{\tau} \\
\xi_{k+2} & =\bar{A}^{2} \xi_{k}+\bar{A} \bar{B}_{v} v_{k}+\bar{B}_{v} v_{k+1}+\left(\bar{A} \bar{B}_{\tau}+\bar{B}_{\tau}\right) \bar{\tau} \\
\vdots & \\
\xi_{k+n} & =\bar{A}^{n} \xi_{k}+\bar{A}^{n-1} \bar{B}_{v} v_{k}+\bar{A}^{n-2} \bar{B}_{v} v_{k+1}+\cdots \\
& +\bar{A}^{2} \bar{B}_{v} v_{k+n-2}+\bar{A} \bar{B}_{v} v_{k+n-2}+\bar{B}_{v} v_{k+n-1} \\
& +\left(\bar{A}^{n-1} \bar{B}_{\tau}+\bar{A}^{n-2} \bar{B}_{\tau}+\cdots+\bar{A} \bar{B}_{\tau}+\bar{B}_{\tau}\right) \bar{\tau}
\end{aligned}
$$

Note that the states at a future moment are dependent on the current states and other sequence terms. And, this can be summarized with a matrix form:

$$
\underset{\rightarrow \mathrm{n}}{\Xi}=G_{\xi} \cdot \xi_{k}+H \cdot \underset{\rightarrow n-1}{V}+W \cdot \mathrm{T}
$$

where,

$$
\underset{\rightarrow \mathrm{n}}{\Xi}=\left[\begin{array}{c}
\xi_{k+1} \\
\xi_{k+2} \\
\xi_{k+3} \\
\vdots \\
\xi_{k+n}
\end{array}\right], G_{\xi}=\left[\begin{array}{c}
\bar{A} \\
\bar{A}^{2} \\
\bar{A}^{3} \\
\vdots \\
\bar{A}^{n}
\end{array}\right], \underset{\rightarrow n-1}{V}=\left[\begin{array}{c}
v_{k} \\
v_{k+1} \\
v_{k+2} \\
\vdots \\
v_{k+n-1}
\end{array}\right], \mathrm{T}=\left[\begin{array}{c}
1 \\
1 \\
1 \\
\vdots \\
1
\end{array}\right] \bar{\tau}
$$




$$
\begin{aligned}
H & =\left[\begin{array}{ccccc}
\bar{B}_{v} & 0 & 0 & \cdots & 0 \\
\bar{A}_{v} \bar{B}_{v} & \bar{B}_{v} & 0 & \cdots & 0 \\
\bar{A}^{2} \bar{B}_{v} & \bar{A}_{v} & \bar{B}_{v} & \cdots & 0 \\
\vdots & \vdots & \vdots & \ddots & \vdots \\
\bar{A}^{n-1} \bar{B}_{v} & \bar{A}^{n-2} \bar{B}_{v} & \bar{A}^{n-3} \bar{B}_{v} & \cdots & \bar{B}_{v}
\end{array}\right] \\
W & =\left[\begin{array}{ccccc}
\bar{B}_{\tau} & 0 & 0 & \cdots & 0 \\
\bar{A}_{\bar{B}} & \bar{B}_{\tau} & 0 & \cdots & 0 \\
\bar{A}^{2} \bar{B}_{\tau} & \bar{A} \bar{B}_{\tau} & \bar{B}_{\tau} & \cdots & 0 \\
\vdots & \vdots & \vdots & \ddots & \vdots \\
\bar{A}^{n-1} \bar{B}_{\tau} & \bar{A}^{n-2} \bar{B}_{\tau} & \bar{A}^{n-3} \bar{B}_{\tau} & \cdots & \bar{B}_{\tau}
\end{array}\right]
\end{aligned}
$$

\subsubsection{Optimal Problem Formulation}

The control goal is to minimize both the lateral deviation from the original course and to achieve a safe heading angle while minimizing control efforts. Therefore, the cost function is defined as the summation of weighted state penalty and control input.

$$
J=\sum_{i=1}^{n+1} \tilde{\xi}_{k+i}^{T} \cdot Q \cdot \tilde{\xi}_{k+i}+\sum_{i=0}^{n} v_{k+i}^{T} \cdot R \cdot v_{k+i}
$$

where, $\tilde{\xi}_{k+i}=\xi_{k+i}-\xi_{k, d e s i r e d}$ and $Q$ and $R$ are diagonal weighting matrices. Here, the same time horizon $(n)$ is used for both state and input sequences. Once the optimal control input sequence $\left(\underset{\rightarrow n-1}{V^{*}}\right)$ is found, only the first control element $\left(v_{k}^{*}\right)$ will be implemented. Equation (5.13) can be rewritten using a more compact notation as follows:

$$
J=\underset{\rightarrow \mathrm{n}}{\tilde{\Xi}} \cdot \operatorname{diag}(Q) \cdot \underset{\rightarrow \mathrm{n}}{\tilde{\Xi}}+\underset{\rightarrow n-1}{V^{T}} \cdot \operatorname{diag}(R) \cdot \underset{\rightarrow n-1}{V}
$$

where, $\operatorname{diag}(Q)=\left[\begin{array}{cccc}Q & 0 & \cdots & 0 \\ 0 & Q & \cdots & 0 \\ \vdots & \vdots & \ddots & \vdots \\ 0 & 0 & \cdots & Q\end{array}\right]$ and $\operatorname{diag}(R)=\left[\begin{array}{cccc}R & 0 & \cdots & 0 \\ 0 & R & \cdots & 0 \\ \vdots & \vdots & \ddots & \vdots \\ 0 & 0 & \cdots & R\end{array}\right]$.

By using Equation (5.12), Equation (5.14) can be rewritten in a quadratic form. 


$$
\begin{aligned}
J & =\underset{\rightarrow n-1}{V}\left(H^{T} Q H+R\right) \underset{\rightarrow n-1}{V}+2 \underset{\rightarrow n-1}{V} H^{T} Q\left(G_{\xi} \tilde{\xi}_{k}+W \mathrm{~T}\right) \\
& +\left(G_{\xi} \tilde{\xi}_{k}\right)^{T} Q\left(G_{\xi} \tilde{\xi}_{k}\right)+2(W \mathrm{~T})^{\mathrm{T}} Q G_{\xi} \tilde{\xi}_{k}+(W \mathrm{~T})^{\mathrm{T}} Q(W \mathrm{~T})
\end{aligned}
$$

Since the last three terms in Equation (5.15) are not affected by $\underset{\rightarrow n-1}{V}$, those terms can be ignored when determining the optimal control set $\left.\underset{\rightarrow n-1}{V^{*}}\right)$.

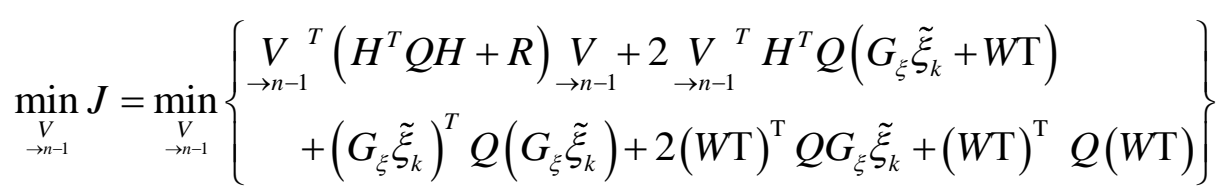

Then,

$$
\min _{\substack{V \\ \rightarrow n-1}} J=\min _{\substack{\rightarrow \\ \rightarrow-1}}\left\{\underset{\rightarrow n-1}{V^{T}}\left(H^{T} Q H+R\right) \underset{\rightarrow n-1}{V}+2 \underset{\rightarrow n-1}{V^{T}} H^{T} Q\left(G_{\xi} \tilde{\xi}_{k}+W T\right)\right\}
$$

This allows the control objective to be formulated in a simpler quadratic form:

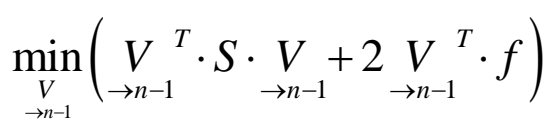

where,

$$
\begin{gathered}
S=H^{T} Q H+R \\
f=H^{T} Q\left(G_{\xi} \tilde{\xi}_{k}+W \mathrm{~T}\right)
\end{gathered}
$$




\subsubsection{Constraints Handling}

Because of the coupling effect of longitudinal-lateral tire forces, the induced lateral tire force $\left(\Delta F_{y, \oplus_{\ominus}}\right)$ generated by braking force $\left(F_{x, \oplus_{\ominus}}\right)$ needs to be considered. Figure 5.4 illustrates the influence of the braking actions on tires, which generate yaw moments calculated from Equations (5.21)-(5.28). $M_{z, \oplus_{\ominus}}$ are the yaw moments generated by $F_{x, \oplus_{\ominus}}$, and $\widetilde{M}_{z, \oplus_{\ominus}}$ are the yaw moments generated by $\Delta F_{y, \oplus_{\ominus}}$.

$$
\begin{gathered}
M_{z, f_{L}}=F_{x, f_{L}} \cdot\left(a \cdot \sin (\delta)-\frac{T_{W}}{2} \cdot \cos (\delta)\right) \\
M_{z, f_{L}}=F_{x, f_{R}} \cdot\left(a \cdot \sin (\delta)+\frac{T_{W}}{2} \cdot \cos (\delta)\right) \\
M_{z, r_{L}}=-F_{x, r_{L}} \cdot \frac{T_{W}}{2} \\
M_{z, r_{R}}=F_{x, r_{R}} \cdot \frac{T_{W}}{2} \\
\tilde{M}_{z, f_{L}}=\Delta F_{y, f_{L}} \cdot\left(a \cdot \cos (\delta)+\frac{T_{W}}{2} \cdot \sin (\delta)\right) \\
\tilde{M}_{z, f_{L}}=\Delta F_{x, f_{R}} \cdot\left(a \cdot \cos (\delta)-\frac{T_{W}}{2} \cdot \sin (\delta)\right) \\
\tilde{M}_{z, r_{L}}=-\Delta F_{x, r_{L}} \cdot b \\
\tilde{M}_{z, r_{R}}=-\Delta F_{x, r_{R}} \cdot b
\end{gathered}
$$

Because of the coupling effect of tire forces, a wheel brake action will affect yaw moment both directly (Equations (5.21)-(5.24)) and indirectly (Equations (5.25)-(5.28)). 


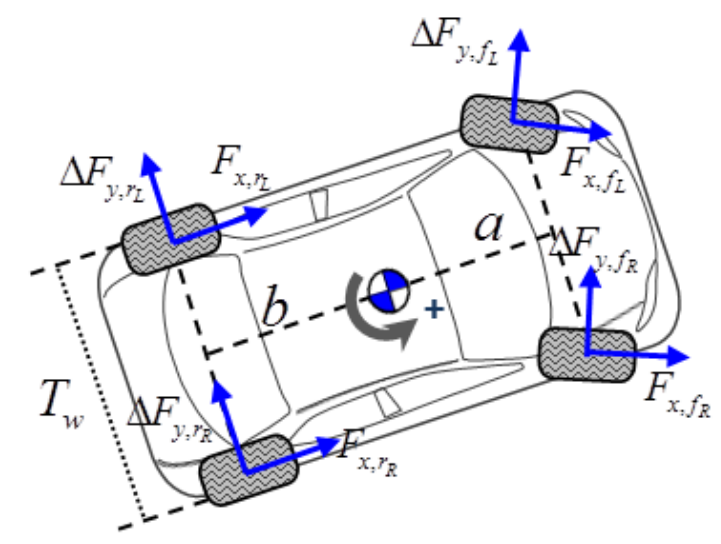

Figure 5.4 Tire force vectors, showing the force effects on vehicle yaw moment with respect to the vehicle $\mathrm{CG}$

Figure 5.5 shows possible ranges of yaw moment $\left(\sum M_{z, \oplus_{\ominus}}:=M_{z, \oplus_{\ominus}}+\widetilde{M}_{z, \oplus_{\ominus}}\right)$ when the wheel slip ratio varies from free rolling $(\lambda=0)$ to fully locked $(\lambda=-1)$ at zero steering angle. The optimization solver will use these bounds to define the feasible control regions. It is noted that the most effective wheel to change yaw moment is determined by the vehicle sideslip angle. For example, there might be a case when the vehicle needs a positive yaw moment and the slip angles of all tires are small positive. In this case, the front-left wheel is the best candidate because the yaw moment on that tire $\left(\sum M_{z, f_{L}}\right)$ shows a greater positive value than others. Similarly, the rear-right wheel is the best to use to generate negative yaw moment generation for the same situation. It should be noted that there exist regions that have limited control authorities for all tires. In Figure 5.5, it can be seen that when the tire slip angle is around $\pm 90^{\circ}$, little yaw moment can be generated, meaning that it is hard to control vehicle yaw motion in those situations.

Feasible regions can also be calculated when the steering angle is non-zero. Figure 5.6 shows the possible yaw moment ranges calculated from Equations (5.21)(5.22) and (5.25)-(5.26). Here, only the yaw moments by front wheel brakes are displayed because we assume the rear wheels are not steered. If MPC needs to solve the optimal solution in non-zero steering angle condition, these feasible regions should be applied. However, in this thesis, only zero steering cases are considered. 

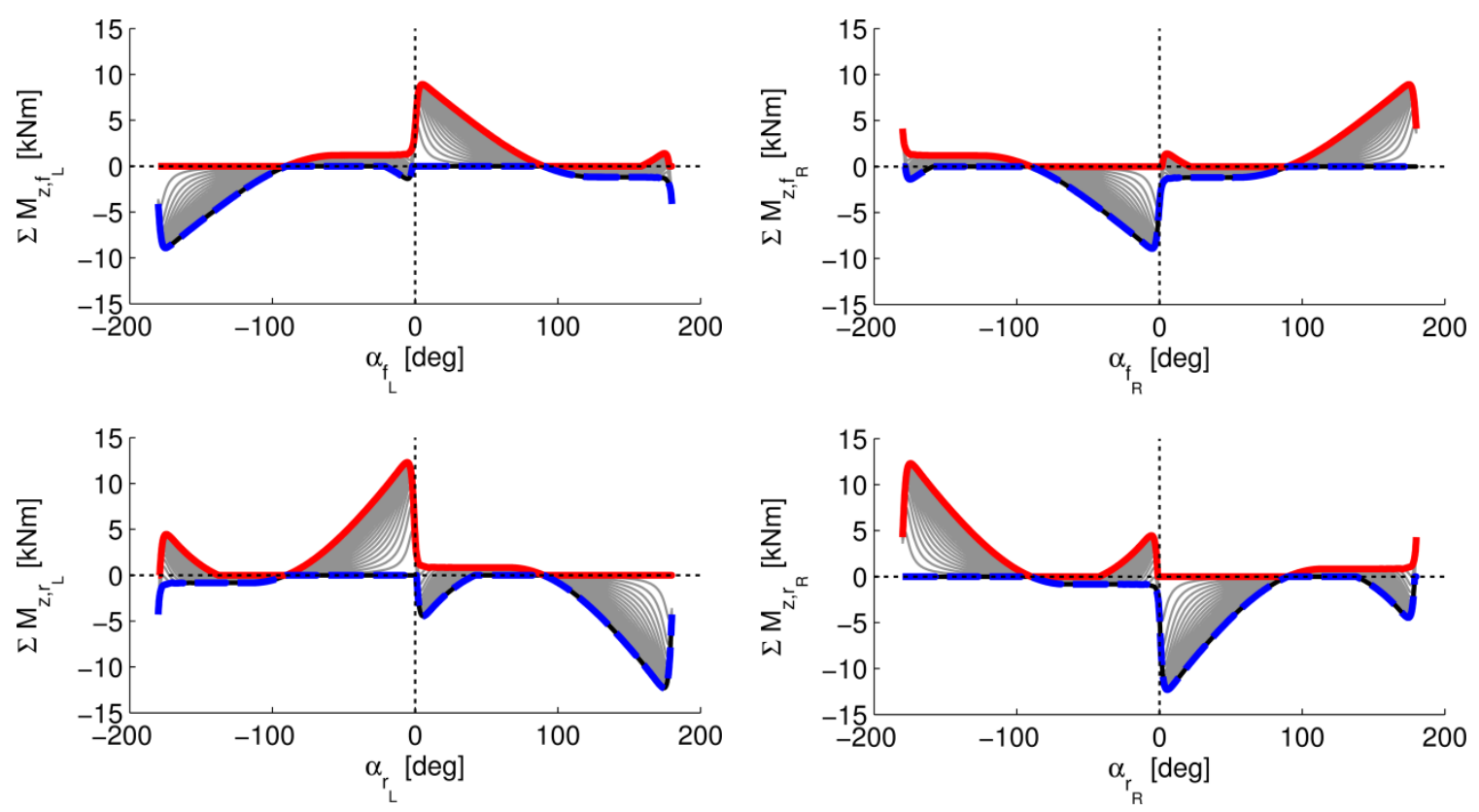

Figure 5.5 Vehicle yaw moments that can be generated by braking at zero steering angle.

The shaded area between red line (upper bound) and blue dashed line (lower bound) depicts achievable region for all possible slip ratio $(-1 \leq \lambda \leq 0)$
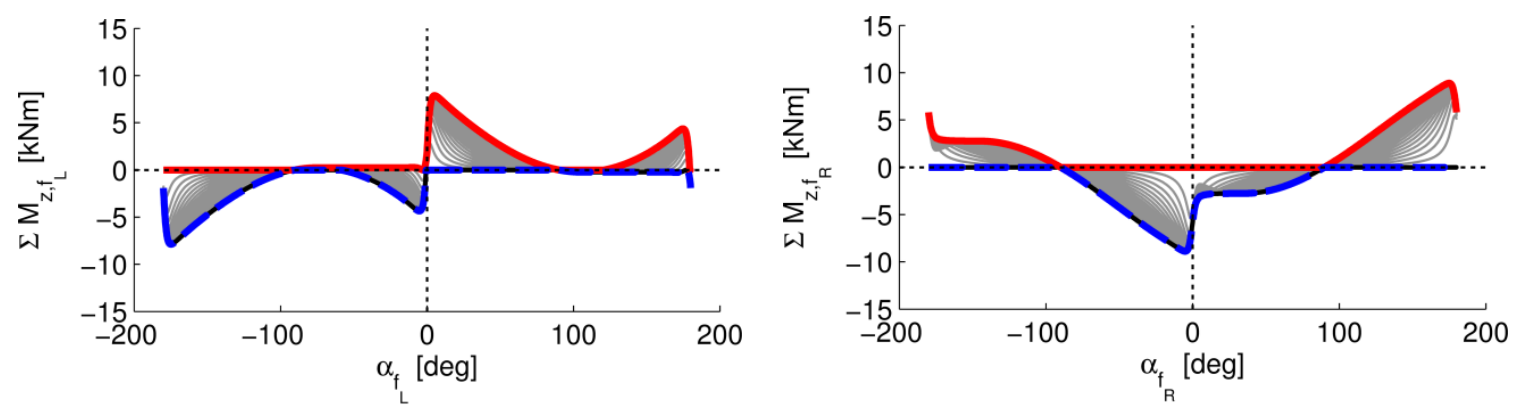

(a) $\delta=20^{\circ}$
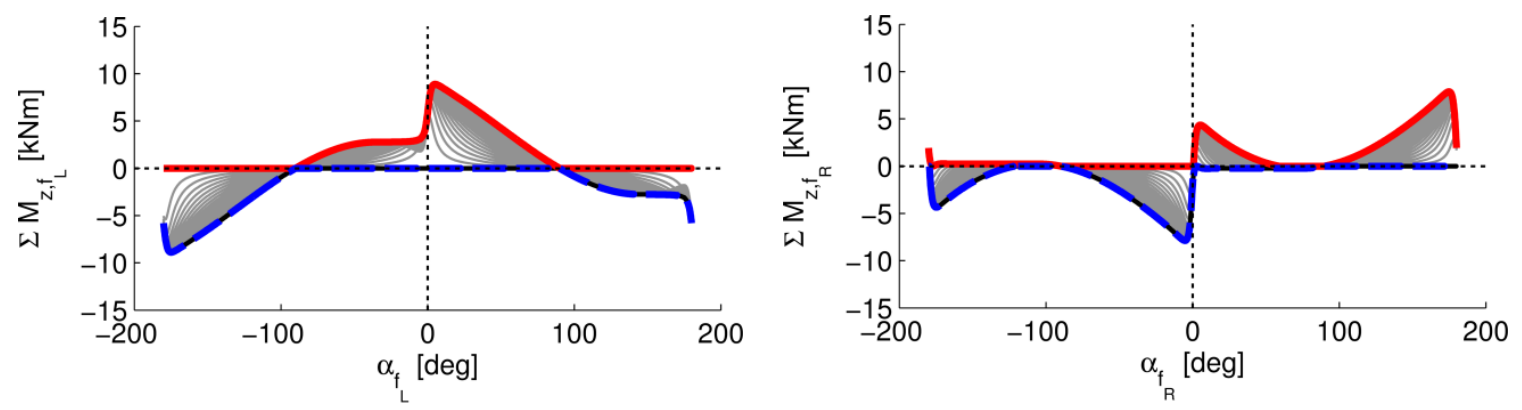

(b) $\delta=-20^{\circ}$

Figure 5.6 Effects of front steering on vehicle yaw moment by braking. Only the front wheels are shown because the rear wheels are not steered. 
Since we are interested in the lateral vehicle motion with respect to its original lane, the vehicle heading angle needs to be considered together with the tire slip angle and slip ratio to find the feasible control regions. In Figure 5.7, the maximum and minimum available forces as functions of the slip angle and heading angle are shown. These properties can be calculated offline and saved as a map. In this way, the optimization calculation considering constraints can be perfomed without taking significant time in realtime.

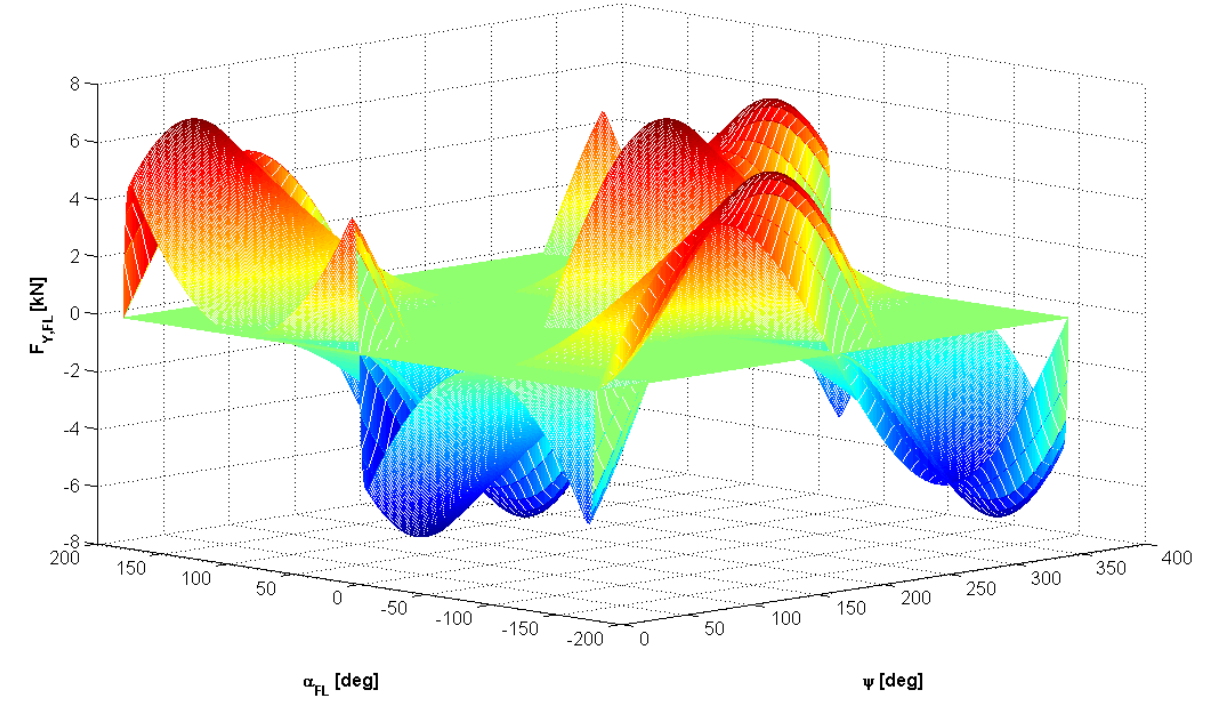

Figure 5.7 Upper and lower bounds of available vehicle lateral forces in the earth-fixed frame.

\subsubsection{Quadratic Programming}

Assuming the upper and lower bounds of the constraints are constant over the MPC problem horizon, which is the same as the horizon $n$. Then, constraints on the control magnitude and rate of change can be defined with the upper and lower bounds in the following inequality equations:

$$
\left[\begin{array}{c}
v_{k}^{l b} \\
v_{k}^{l b} \\
\vdots \\
v_{k}^{l b}
\end{array}\right] \leq\left[\begin{array}{c}
v_{k} \\
v_{k+1} \\
\vdots \\
v_{k+n-1}
\end{array}\right] \leq\left[\begin{array}{c}
v_{k}^{u b} \\
v_{k}^{u b} \\
\vdots \\
v_{k}^{u b}
\end{array}\right]
$$




$$
\left[\begin{array}{c}
\Delta v_{k}^{l b} \\
\Delta v_{k}^{l b} \\
\vdots \\
\Delta v_{k}^{l b}
\end{array}\right] \leq\left[\begin{array}{c}
v_{k}-v_{k-1} \\
v_{k+1}-v_{k} \\
\vdots \\
v_{k+n}-v_{k+n-1}
\end{array}\right] \leq\left[\begin{array}{c}
\Delta v_{k}^{u b} \\
\Delta v_{k}^{u b} \\
\vdots \\
\Delta v_{k}^{u b}
\end{array}\right]
$$

Note that the control limits are constant over the prediction horizon $n$, but vary at every time step $k$. This is because the ranges of the virtual controls change with tire slip angle and heading angle. In other words, the constraints are functions of the states and control inputs. Equation (5.29) can be rewritten in terms of a single set of linear inequalities:

$$
\left[\begin{array}{c}
\mathrm{I} \\
-\mathrm{I}
\end{array}\right] \cdot\left[\begin{array}{llll}
v_{k} & v_{k+1} & \cdots & v_{k+n-1}
\end{array}\right]^{T} \leq\left[\begin{array}{c}
V_{k}^{u b} \\
V_{k}^{l b}
\end{array}\right] \text {, or }\left[\begin{array}{c}
\mathrm{I} \\
-\mathrm{I}
\end{array}\right] \cdot \underset{\rightarrow n-1}{V} \leq\left[\begin{array}{c}
V_{k}^{u b} \\
V_{k}^{l b}
\end{array}\right]
$$

where, I is an identity matrix, $V_{k}^{u b}=\left[\begin{array}{llll}v_{k}^{u b} & v_{k}^{u b} & \cdots & v_{k}^{u b}\end{array}\right]^{T}$ and $V_{k}^{l b}=\left[\begin{array}{llll}v_{k}^{l b} & v_{k}^{l b} & \cdots & v_{k}^{l b}\end{array}\right]^{T}$.

For the rate limit in the control sequence, the equation (5.30) can also be rewritten:

$$
\left[\begin{array}{c}
C_{\Delta} \\
-C_{\Delta}
\end{array}\right] \cdot \underset{\rightarrow n-1}{V} \leq\left[\begin{array}{c}
\Delta V_{k}^{u b}+E \cdot v_{k-1} \\
-\Delta V_{k}^{l b}-E \cdot v_{k-1}
\end{array}\right]
$$

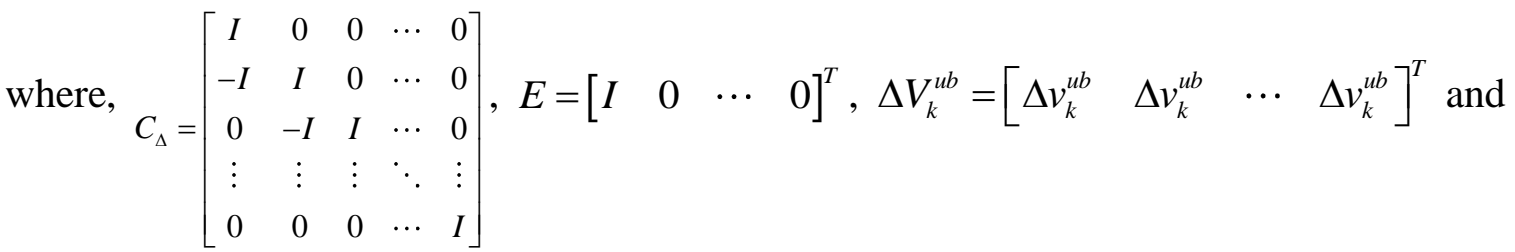
$\Delta V_{k}^{l b}=\left[\begin{array}{llll}\Delta v_{k}^{l b} & \Delta v_{k}^{l b} & \cdots & \Delta v_{k}^{l b}\end{array}\right]^{T}$.

After combining Equations (5.31) and (5.32) into one matrix form, one can obtain a simplified constraint expression as: $h(\underset{\rightarrow \mathrm{n}}{\Xi} \underset{\rightarrow n-1}{V})<0$.

Then, we obtain a general quadratic programming problem with the inequality constraints, which can be solved using the MATLAB Optimization Toolbox (quadprog).

$$
\min _{\substack{V \\ \rightarrow n-1}} J\left(\xi_{k}, \underset{\rightarrow n-1}{V}\right) \quad \text { subject to } \quad h(\underset{\rightarrow n \rightarrow n-1}{\Xi} \underset{, V}{V})
$$




\subsection{Optimal Allocation Problem}

The control laws derived in the previous section computes the optimal virtual controls. As shown in Figure 5.8, the virtual control commands are fed to the wheel brake optimal allocation module to determine the effective physical controls. Since we assume that the control actuators of the system are the individual wheel brakes, the outputs of this module are longitudinal wheel brake forces of each tire. Specifically, the objective of this module is to find optimal control $u_{k}^{*} \in \mathcal{R}^{4}$, from the first step of the virtual control sequence $v_{k}^{*} \in \mathcal{R}^{2}$.

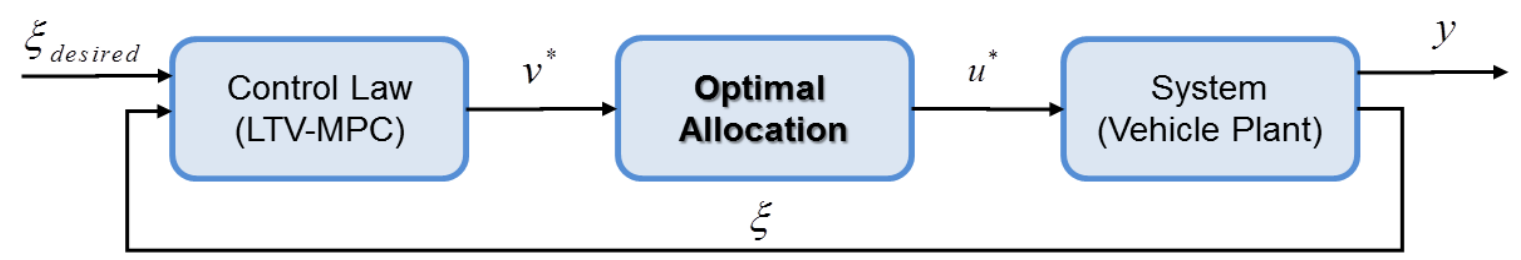

Figure 5.8 Signal flows for the optimal allocation algorithm

\subsubsection{Tire Force Relationship}

Equation (5.4) can be rewritten as:

$$
g(\xi, u, t)=B_{u} \cdot u+W_{d} \cdot d
$$

where, $d=\left[\begin{array}{llll}\Delta F_{y, f_{L}} & \Delta F_{y, f_{R}} & \Delta F_{y, r_{L}} & \Delta F_{y, r_{R}}\end{array}\right]$,

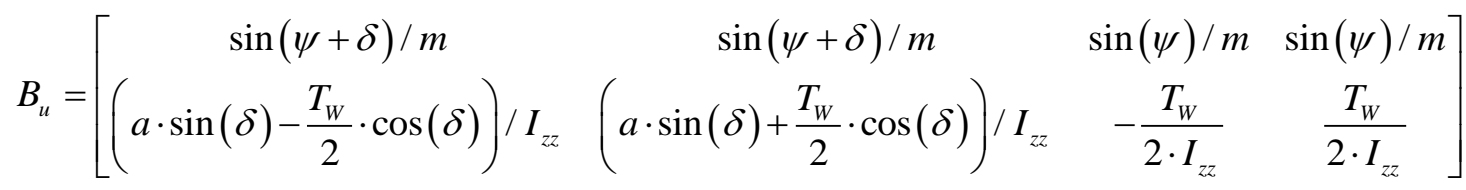

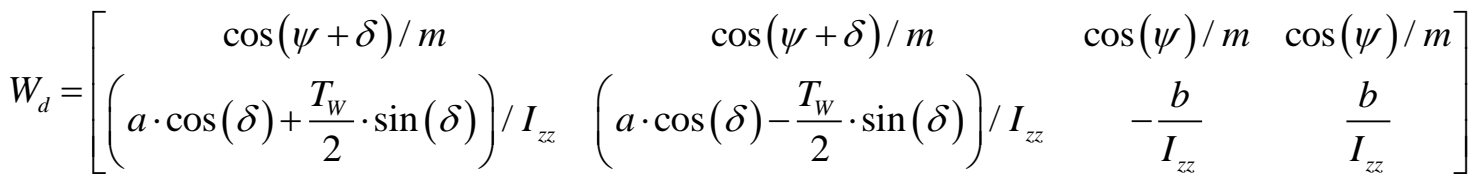


To replace the induced lateral force term $d$ as a function of $u$, we utilize the force coupling effect. The tire forces are constrained by an enveloping curve, called a friction circle or friction ellipse [81]. The Magic Formula used in this research inherently describes combined tire forces within this friction ellipse, and this profile can be constructed as a linear function by manipulating the tire model equations. From the definition in Equation (2.13), one can analytically obtain the gradients of forces $\left(F_{x}, F_{y}\right)$ with respect to $\lambda\left(=s_{x}\right)$.

$$
\begin{aligned}
& \frac{\partial F_{x}}{\partial s_{x}}=\frac{F_{p} \cdot s_{y}^{2}}{\left(s_{x}^{2}+s_{y}^{2}\right)^{3 / 2}} \cdot \Phi\left(\frac{C_{\alpha}\|s\|}{F_{p}}\right)+\Phi^{\prime}\left(\frac{C_{\alpha}\|s\|}{F_{p}}\right) \cdot \frac{C_{\alpha} \cdot s_{x}^{2}}{s_{x}^{2}+s_{y}^{2}} \\
& \frac{\partial F_{y}}{\partial s_{x}}=\frac{-F_{p} \cdot s_{x} \cdot s_{y}}{\left(s_{x}^{2}+s_{y}^{2}\right)^{3 / 2}} \cdot \Phi\left(\frac{C_{\alpha}\|s\|}{F_{p}}\right)+\Phi^{\prime}\left(\frac{C_{\alpha}\|s\|}{F_{p}}\right) \cdot \frac{C_{\alpha} \cdot s_{x} \cdot s_{y}}{s_{x}^{2}+s_{y}^{2}}
\end{aligned}
$$

Equation (5.21) is then used to relate $F_{x}$ and $\Delta F_{y}$ :

$$
\Gamma:=\frac{\partial \Delta F_{y}}{\partial F_{x}}=\frac{\partial\left(F_{y}-F_{y}^{0}\right)}{\partial s_{x}} \cdot \frac{\partial s_{y}}{\partial F_{x}}=\frac{\partial F_{y}}{\partial s_{x}} \cdot \frac{\partial s_{y}}{\partial F_{x}}
$$

This matrix $\Gamma^{4 \times 4}$ is a diagonal matrix which maps the tire longitudinal forces onto the lateral forces, $\left[\Delta F_{y}\right]=\Gamma \cdot\left[F_{x}\right]$ :

$$
\Gamma=\operatorname{diag}\left[\frac{\partial\left(\Delta F_{y, f_{L}}\right)}{\partial F_{x, f_{L}}} \frac{\partial\left(\Delta F_{y, f_{R}}\right)}{\partial F_{x, f_{R}}} \frac{\partial\left(\Delta F_{y, r_{L}}\right)}{\partial F_{x, r_{L}}} \frac{\partial\left(\Delta F_{y, r_{R}}\right)}{\partial F_{x, r_{R}}}\right]
$$

Then, Equation (5.34) becomes:

$$
B_{v} \cdot v=\left(B_{u}+W_{d} \cdot \Gamma\right) \cdot u
$$




\subsubsection{Allocation Algorithm}

The optimal allocation problem solves the linear relationship, Equation (5.39), in the least-squares sense subject to the actuator saturation limit. Since the actuators are

wheel brake forces, the control bounds are $F_{x}^{\min } \leq u \leq F_{x}^{\max }$. Because we consider brake control actions only, the value $u$ should have negative values. We employ the following least-squares problem for optimal allocation:

$$
\min _{\mathrm{u}_{k}}\left(\left\|u_{k}\right\|_{2}^{2}+w \cdot\left\|\left(B_{u}+W_{d} \cdot \Gamma\right) \cdot \mathrm{u}_{k}-B_{v} \cdot v_{k}^{*}\right\|_{2}^{2}\right)
$$

$$
\text { Subject to } \quad u^{\min }\left(\xi_{k}\right) \leq u_{k} \leq u^{\max }\left(\xi_{k}\right)
$$

where, the parameter $w$ is the positive weighting factor chosen to achieve the relationship in Equation (5.39) as close as possible.

\subsection{Simulation Results with Control Actions}

The results in this section show the performance of the proposed secondary collision mitigation function. The simulated scenario, as shown in Figure 1.2, is that two vehicles are involved in a minor sideswipe. Then the collision leads the vehicle to travel to the opposite traffic lane if no control is applied. All state measurements such as position, speed, yaw rate, and heading angle are assumed to be available and accurate, and actuator delays are assumed to be non-existing. In addition, the first impact is assumed to be minor so that all actuators function normally. The vehicle is assumed to be a big SUV in CarSim $\left(m=2450 \mathrm{~kg}, I_{z}=4946 \mathrm{kgm}^{2}, a=1.105 \mathrm{~m}, b=1.745 \mathrm{~m}\right)$. The vehicle is assumed to travel with an initial longitudinal speed of $30 \mathrm{~m} / \mathrm{s}$ (67 mph, $108 \mathrm{kph}$ ) on a flat and straight road. The first impact is assumed to result in the initial conditions: lateral speed $5.0 \mathrm{~m} / \mathrm{s}$, heading angle $9.2^{\circ}$, and yaw rate $114.6^{\circ} / \mathrm{sec}$. The time period for linearization and MPC time horizon are $0.2 \mathrm{sec}$, and the sampling time to run a simulation is $0.01 \mathrm{sec}$. Simulated four vehicle trajectories are presented in Figure 5.9 for comparison. It is shown that the vehicle with the proposed controller settles to a safe final heading angle of $180^{\circ}$ and returns to the original lane. In contrast, the vehicles without 
braking intervention and with other control strategies depart from their original lane, and can be broadsided by vehicles in other lanes.

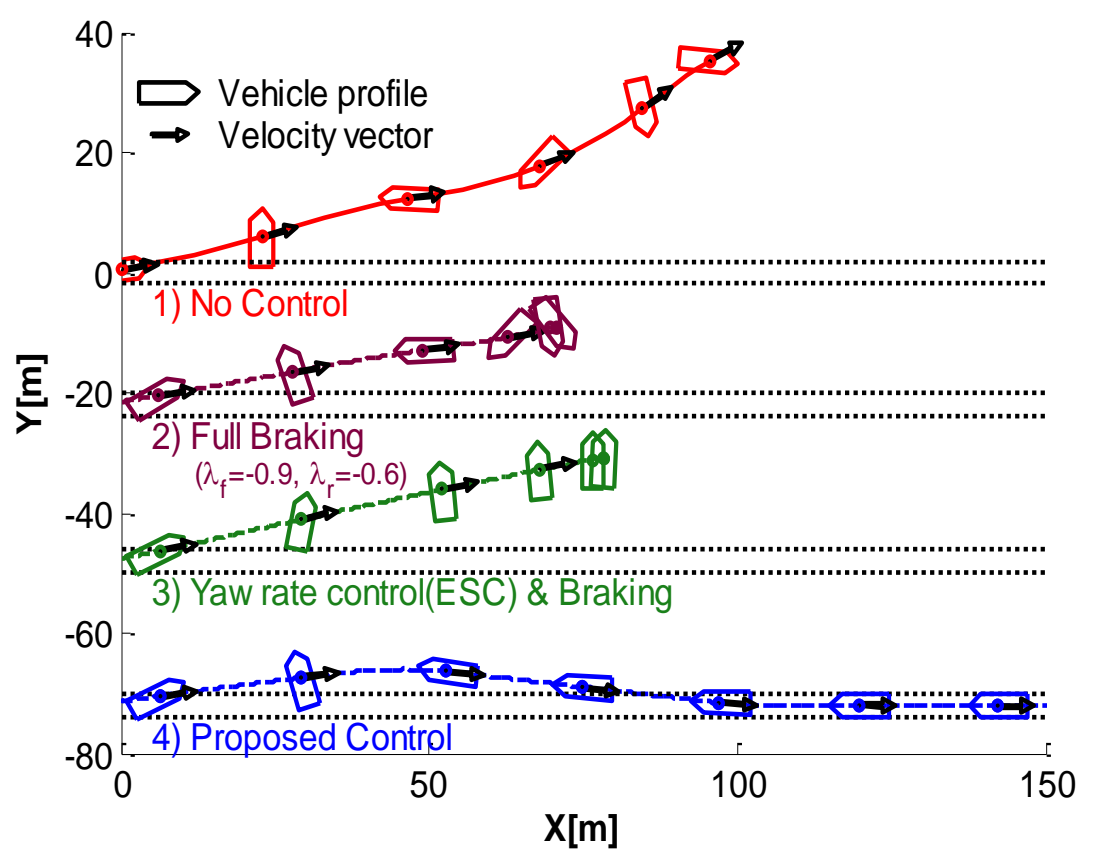

Figure 5.9 Vehicle trajectories under three control strategies. (Vehicle sizes are doubled.)
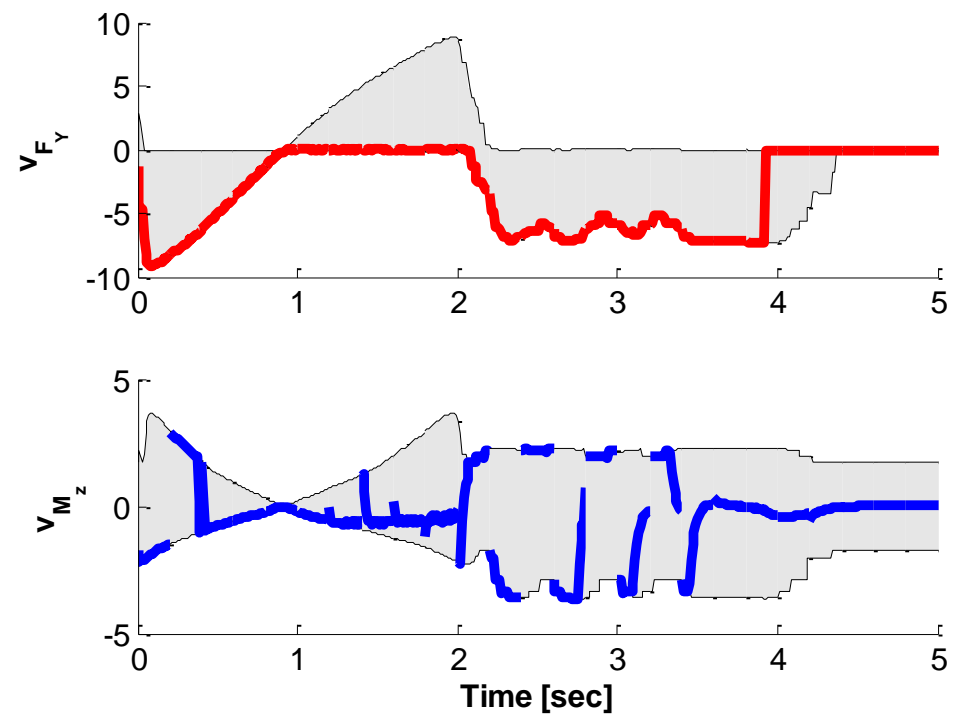

Figure 5.10 Virtual control inputs from the proposed control strategy. Feasible boundaries are shown by gray shaded regions. Virtual controls solved by MPC are shown in bold lines. 
In the case of yaw rate control, the brake control actions to change the vehicle yaw motion are very limited at the end of the maneuver because the tire slip angles are all around $\pm 90^{\circ}$ as shown in Figure 5.5. Moreover, attempting to drive the vehicle to the original lane (i.e. heading angle to the original $0^{\circ}$ ) can cause a large lateral deviation [82].

The virtual control bounds and control results from the LTV-MPC are shown in Figure 5.10. Individual wheel control commands are shown in Figure 5.11. In Figure 5.12, the capability of the proposed control strategy is evaluated under a few different initial conditions. The conditions are similar to the one illustrated in Figure 5.9, but with different values for initial yaw rate and heading angle. Overall, it is seen that the proposed control reduces the maximum lane deviations and brings the vehicle back to the original lane with the desired heading angle of either $180^{\circ}$ or $360^{\circ}$.
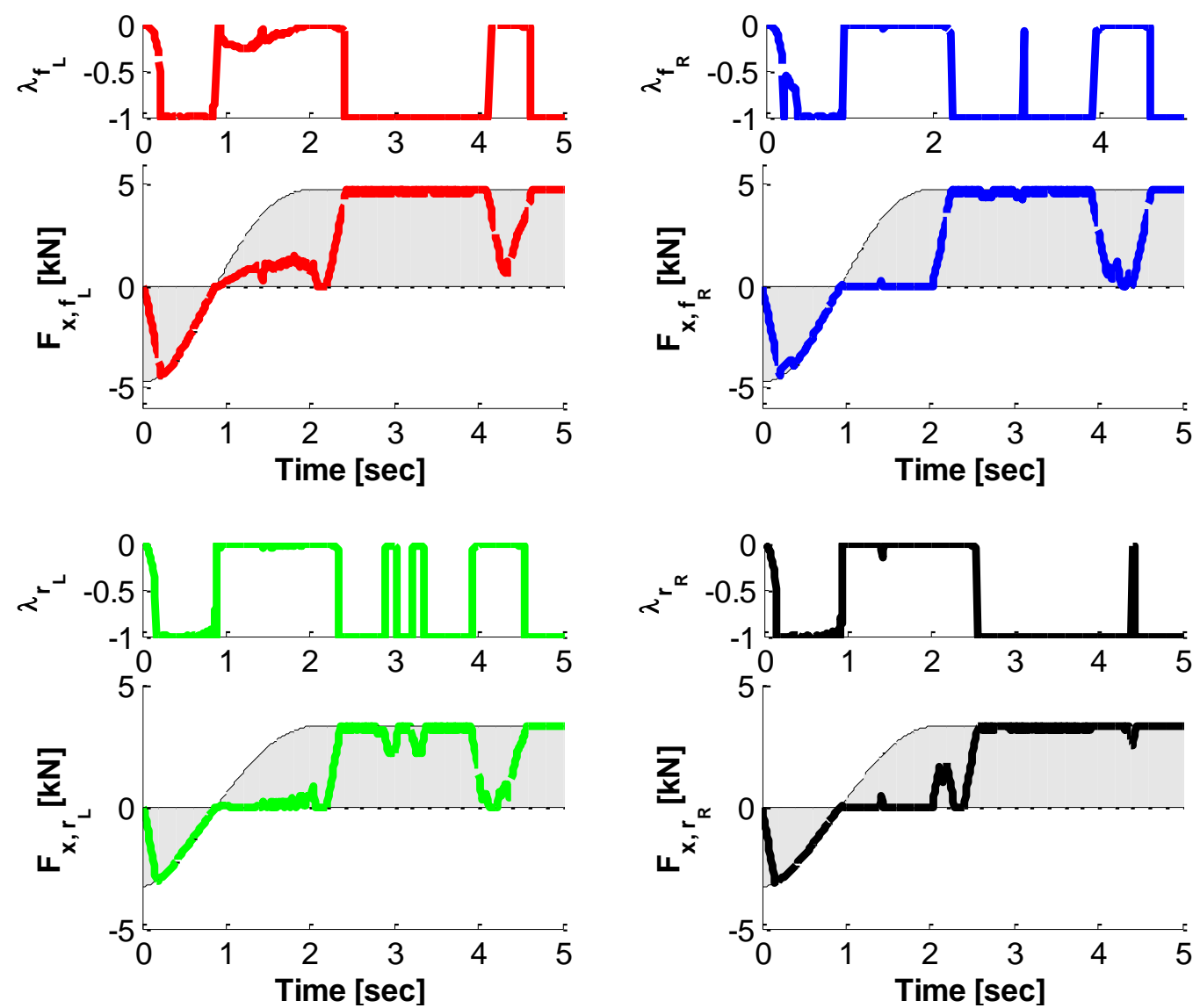

Figure 5.11 Longitudinal tire slip ratio commands and corresponding longitudinal forces calculated by the optimal allocation problem. The gray shaded regions depict the feasible control bounds. 


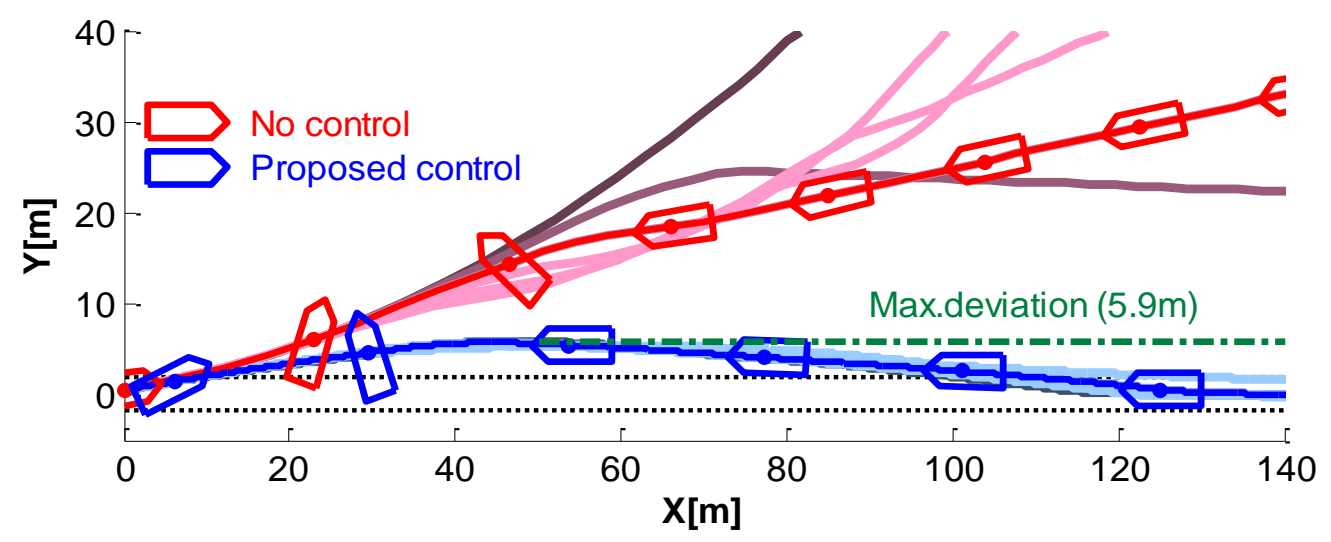

(a) Initial yaw rate: $80 \sim 120^{\circ} / \mathrm{s}$ and heading angle: $9 \sim 17^{\circ}$

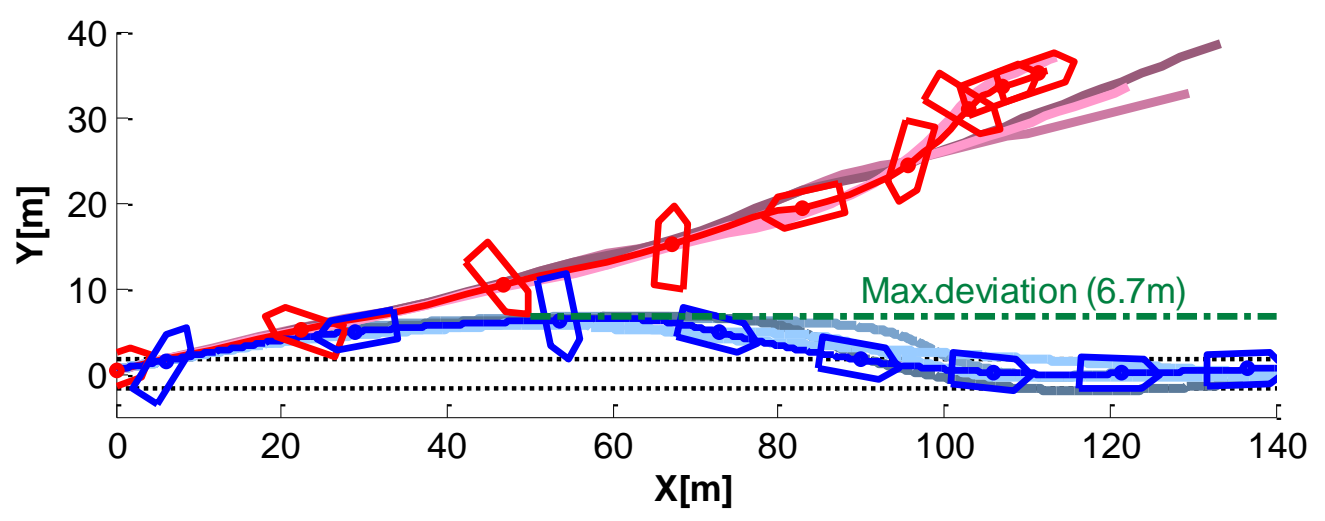

(b) Initial yaw rate: 130 200\% and heading angle: $18 \sim 23^{\circ}$

Figure 5.12 Vehicle trajectories under several initial conditions representing different levels of impact: yaw rate $80 \sim 200^{\circ}$ s and heading angle $9 \sim 23^{\circ}$ with fixed lateral speed $(5 \mathrm{~m} / \mathrm{s})$ and longitudinal speed $(30 \mathrm{~m} / \mathrm{s})$ 


\subsection{Rule-based Control for Real-time System Implementation}

A rule-based control uses if-then rules that do not require complex mathematical models [83]. The controller is run by explicit relationships between the defined control rules and the system responses $[84,85]$. Although the control performance is usually not optimal, it can be tuned to achieve reasonable performance if a benchmark control is available. One major benefit of the rule based controls is that they usually have very light computational load. In the following, the rule based control is constructed to mimic the optimal control behavior generated by the LTV-MPC. This involves knowledge extraction from the LTV-MPC control results.

\subsubsection{Learning from the LTV-MPC Control Sequence and Rule Extraction}

Three optimal control results calculated by LTV-MPC are shown in Figure 5.13Figure 5.15. At the beginning in Figure 5.13, all wheels are locked until the heading angle reaches $90^{\circ}$. Then, one of the wheels, which can generate opposite directional yaw motion by braking, takes a dominant role to regulate the spin motion of the vehicle until the heading angle approaches $180^{\circ}$. Because the yaw rate is not low enough at $180^{\circ}$ heading angle, another wheel locking action is performed, followed by the spin motion regulation by one of the wheel brakes when the heading angle passed $270^{\circ}$. Finally, the differential brake control is applied to stabilize the vehicle motion around $360^{\circ}$.

Note that there are some repeating patterns in Figure 5.14 and Figure 5.15, as well. Shaded regions in Figure 5.13-Figure 5.15 are roughly divided in colors (Red, Blue, and Green) based on the heading angles which are multiples of $90^{\circ}$. It is noted that the pattern from heading angle $0^{\circ}$ to $180^{\circ}$ is similar to the one from $180^{\circ}$ to $360^{\circ}$ and from $360^{\circ}$ to $540^{\circ}$. These switching patterns are also shown in other test examples, and it was found that the switching conditions are dependent on vehicle heading angle, yaw rate, and vehicle longitudinal speed. By looking at each vehicle motion pattern, corresponding brake control actions are identified, and modes and specific switching conditions are assigned. 

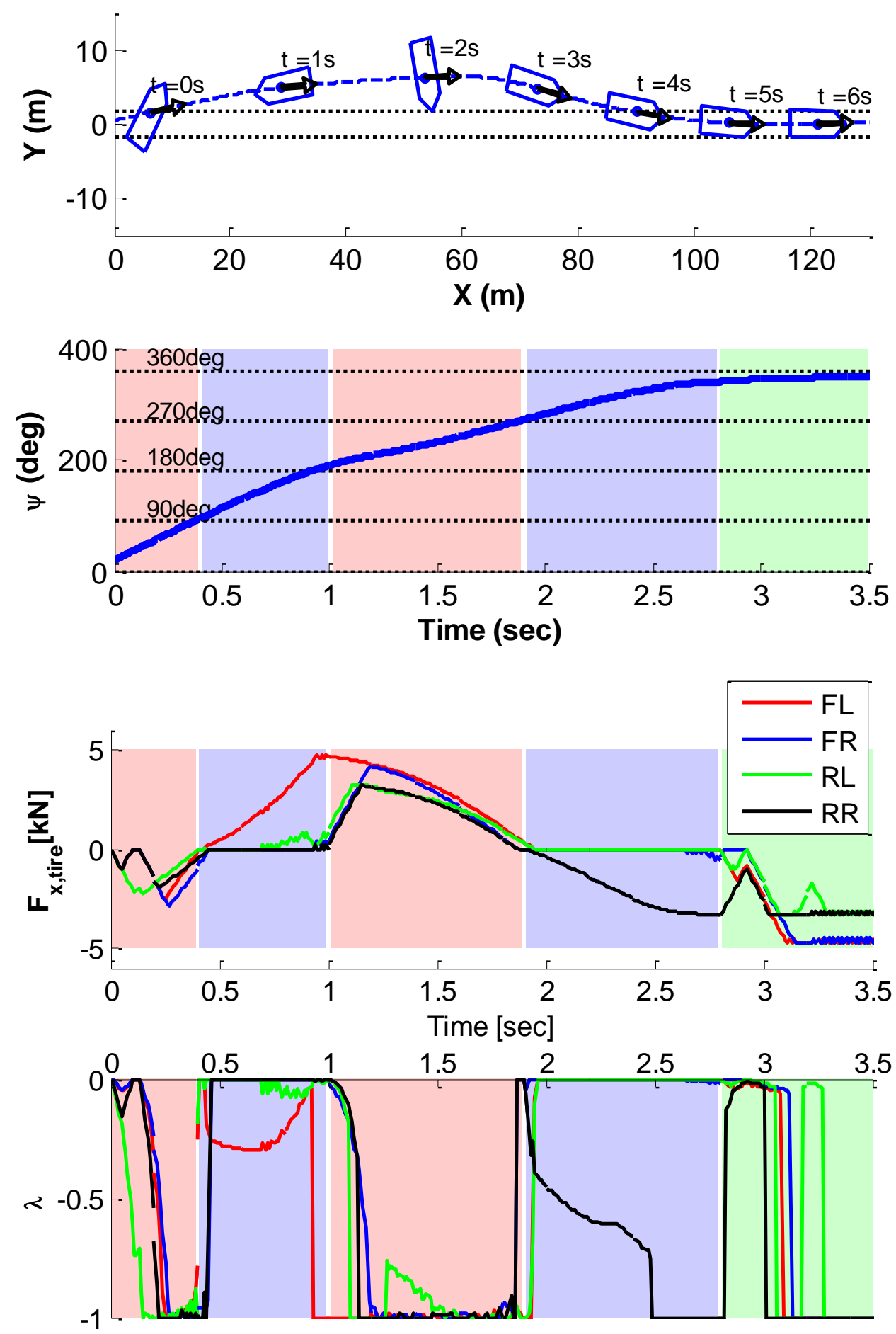

Figure 5.13 Vehicle motion trajectories and brake control sequences from the LTV-MPC (Final heading angle: $360^{\circ}$ ) 

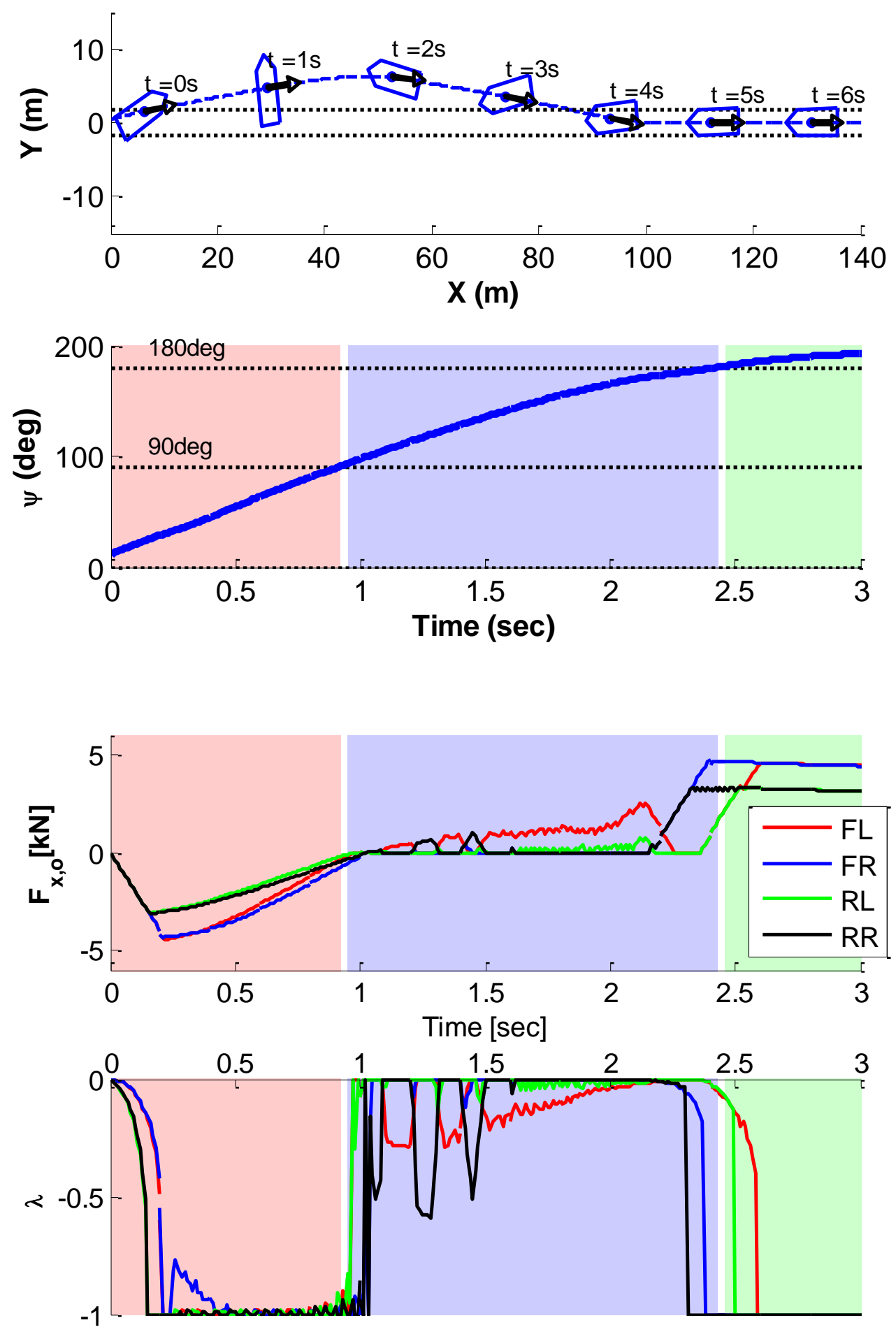

Figure 5.14 Vehicle motion trajectories and brake control sequences from the LTV-MPC (Final heading angle: $180^{\circ}$ ) 

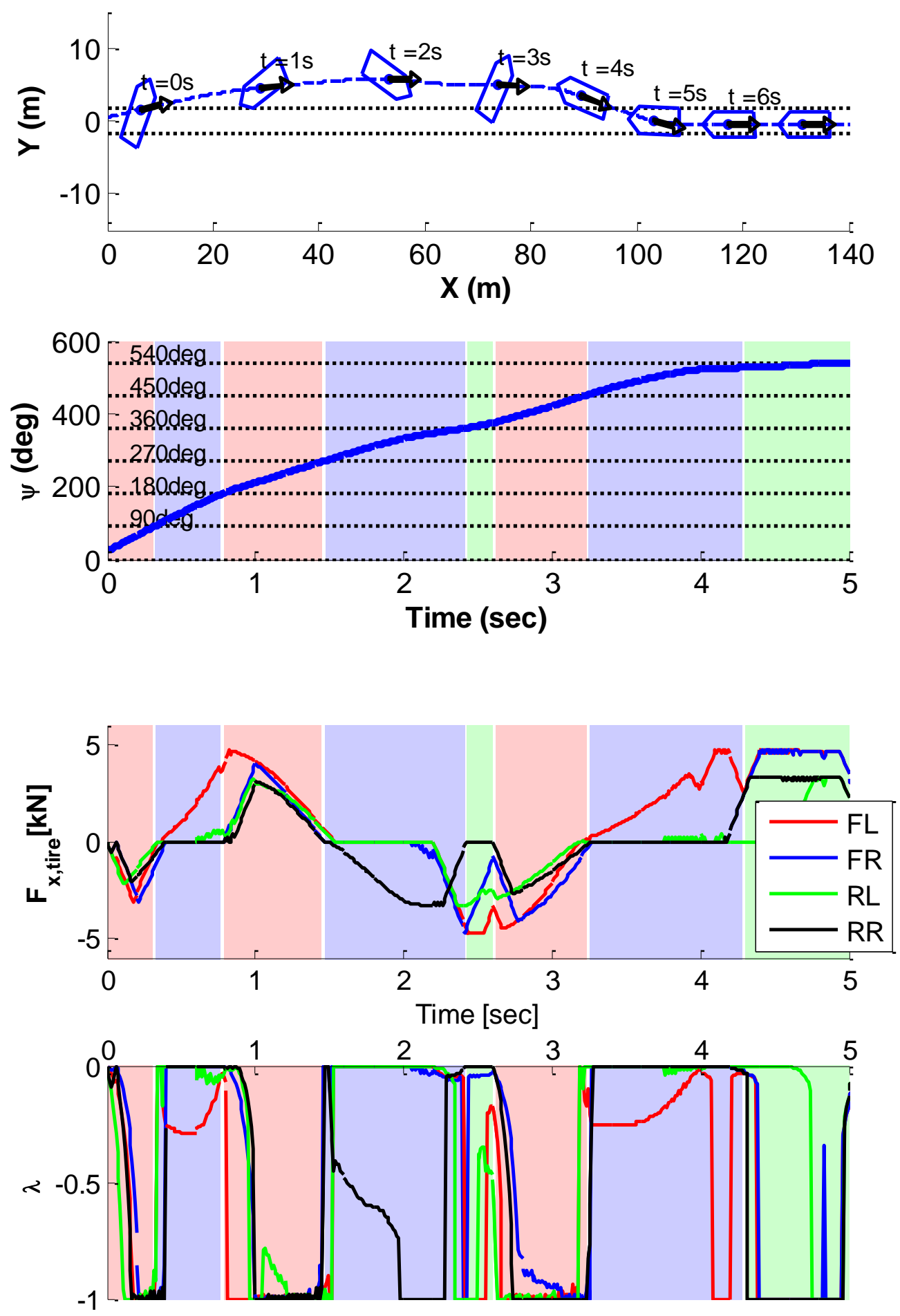

Figure 5.15 Vehicle motion trajectories and brake control sequences from the LTV-MPC (Final heading angle: $540^{\circ}$ ) 
In Figure 5.16, which is zoomed-in view to Figure 5.13, two brake control modes are observed. Rear wheels locking increases both yaw rate and lateral displacement (Notice $V_{Y}$ and $\omega_{z}$ at $t=0.1 \sim 0.2$ seconds in Figure 5.16). Although this mode sacrifices lateral displacement, it is a useful mode to promptly pass the heading angles around $90^{\circ}$ and $270^{\circ}$, which are known as hazardous heading angles to introduce broadside impact. All wheels locking mode allows for the vehicle to reduce both the lateral displacement and the yaw rate. This mode is beneficial to reduce the lateral deviation in high heading angle situations.
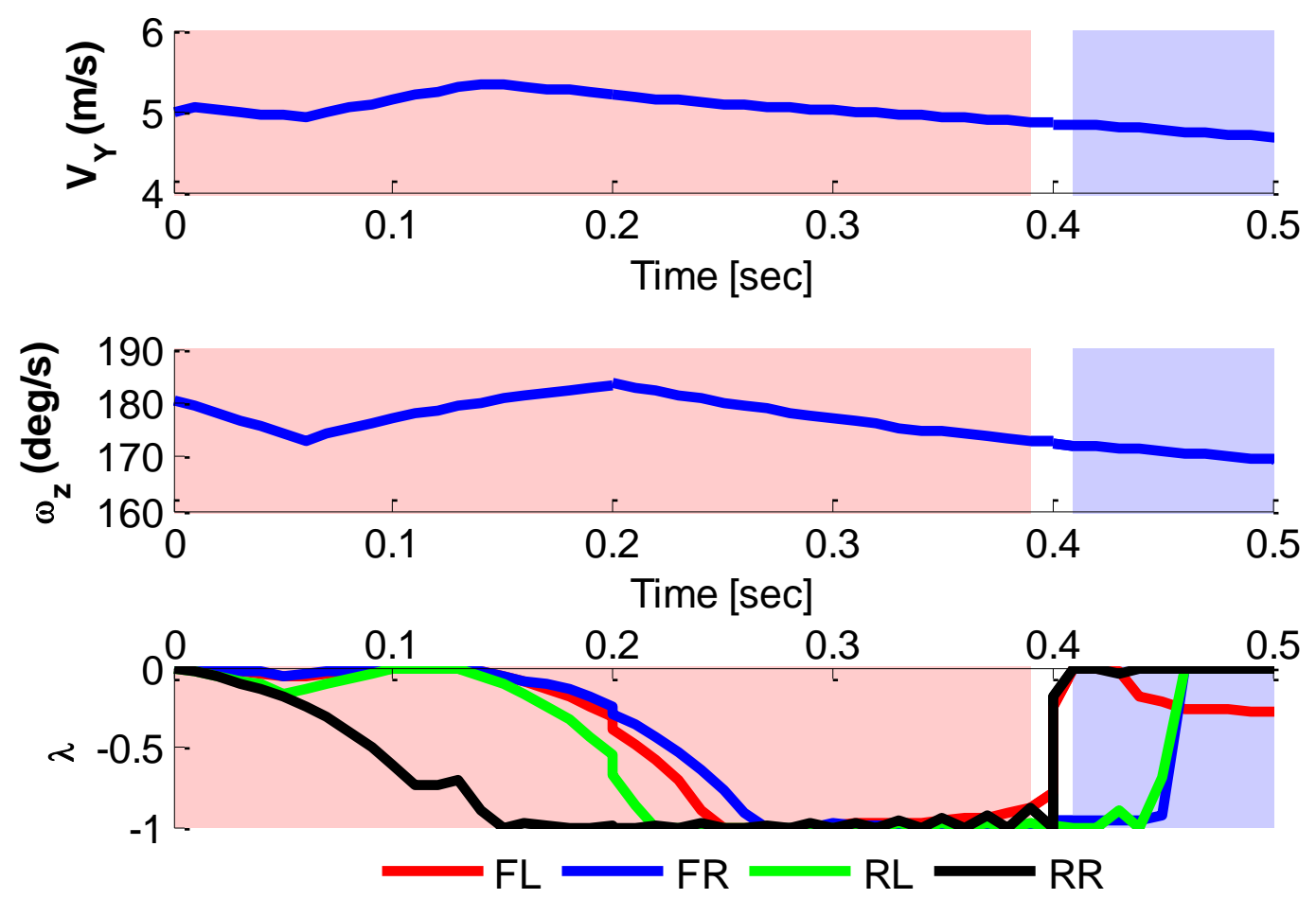

Figure 5.16 Vehicle dynamic motion with brake controls (Zoomed in from Figure 5.13) 


\subsubsection{Controller Configuration}

Based on the review of optimal control results, the following five modes are defined: (1) Wheel locking mode, (2) Yaw rate regulation mode, (3) Yaw angle regulation mode, (4) Stabilization mode, and (5) No control action mode. Small lateral deviation performance is achieved by the wheel locking mode. Higher yaw rate can be achieved with the yaw rate regulation mode, which applies rear wheel braking (front wheel braking when the vehicle heading is reversed, at $180^{\circ}$ ). The yaw angle control mode regulates the heading angle around multiples of $180^{\circ}$. Stabilization mode, which performs the same differential brake control action as ESC, minimizes yaw rate error and sideslip angle. Based on this concept, a rule-based control structure is designed. Figure 5.17 shows the structure of switching modes, where each mode produces wheel brake forces to control the vehicle.

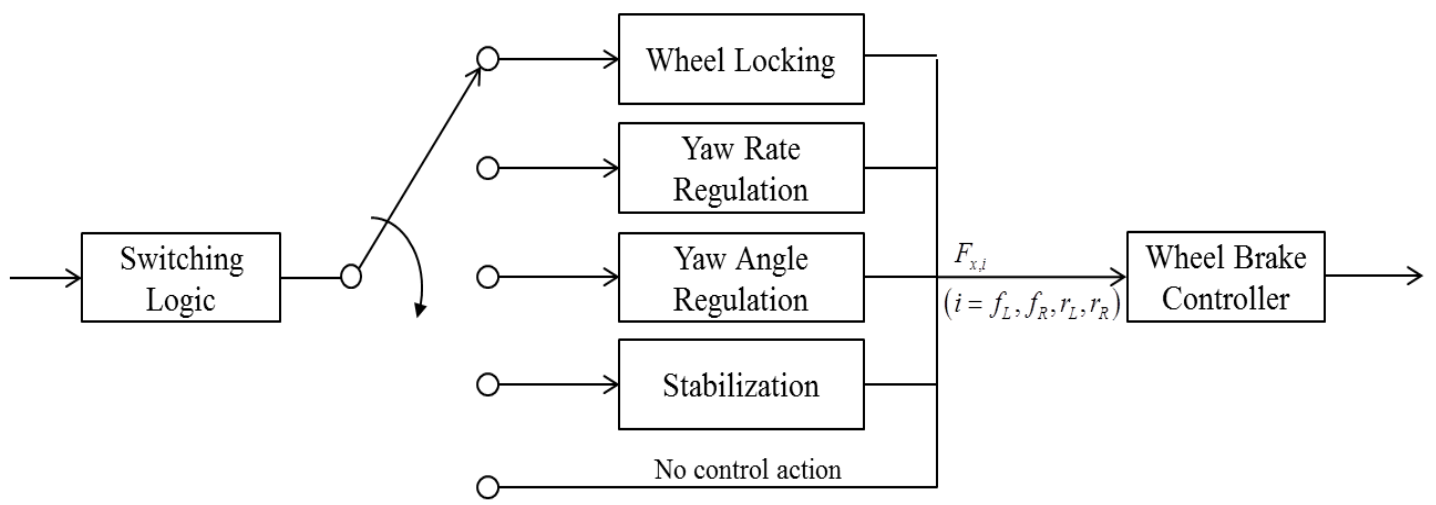

Figure 5.17 Switching structure for the rule-based control

The signal required for switching is the heading angle of the vehicle. Before examining the rule, the heading angle needs to be converted in a manageable range, in $(-2 \pi, 2 \pi)$. We apply modulo operator shown in Equation (5.42) to compute the remainder resulting from division with $2 \pi$.

$$
\psi_{m}=\psi-D_{2 \pi} \cdot\left[\frac{\psi}{D_{2 \pi}}\right]
$$

where, [.] is a floored division, and a modulo heading angle $\left(\psi_{m}\right)$ is the remainder of the division. 
Control action in each mode is described in Table 5.1, and conditions for switching modes are presented in

Table 5.2.

Table 5.1 Modes and control actions

\begin{tabular}{|c|c|c|}
\hline Mode & Actions & Control Objective \\
\hline $\begin{array}{l}\text { (1) Wheel Locking } \\
\text { Control }\end{array}$ & $F_{x, i}=F_{x, \text { tire-max }, i} \quad\left(i=f_{L}, f_{R}, r_{L}, r_{R}\right)$ & $\begin{array}{l}\text { Reduce lateral course } \\
\text { deviation }\left(F_{x, \text { tire-max }, i} \text { are }\right. \\
\text { peak values of the } \\
\text { longitudinal tire force })\end{array}$ \\
\hline $\begin{array}{l}\text { (2) Yaw Rate } \\
\text { Regulation }\end{array}$ & $\begin{array}{l}\text { If }-\pi / 2 \leq \psi_{m} \leq \pi / 2 \\
\left.\qquad \begin{array}{llll}F_{x, f_{L}} & F_{x, f_{R}} & F_{x, r_{L}} & F_{x, r_{R}}\end{array}\right] \\
\quad=\left[\begin{array}{lllll}0 & 0 & F_{x, \text { tire }- \text { max }, r_{L}} & F_{x, \text { tire-max }, r_{R}}\end{array}\right] \\
\text { Else } \\
\left.\qquad \begin{array}{lllll}F_{x, f_{L}} & F_{x, f_{R}} & F_{x, r_{L}} & F_{x, r_{R}}\end{array}\right] \\
=\left[\begin{array}{lllll}F_{x, \text { tire-max }, f_{L}} & F_{x, \text { tire-max }, f_{R}} & 0 & 0\end{array}\right]\end{array}$ & $\begin{array}{l}\text { Increase yaw rate to get } \\
\text { through the limited control } \\
\text { region at around } 90^{\circ} \text { and } \\
270^{\circ} \text { heading angles. }\end{array}$ \\
\hline $\begin{array}{l}\text { (3) Yaw Angle } \\
\text { Control }\end{array}$ & 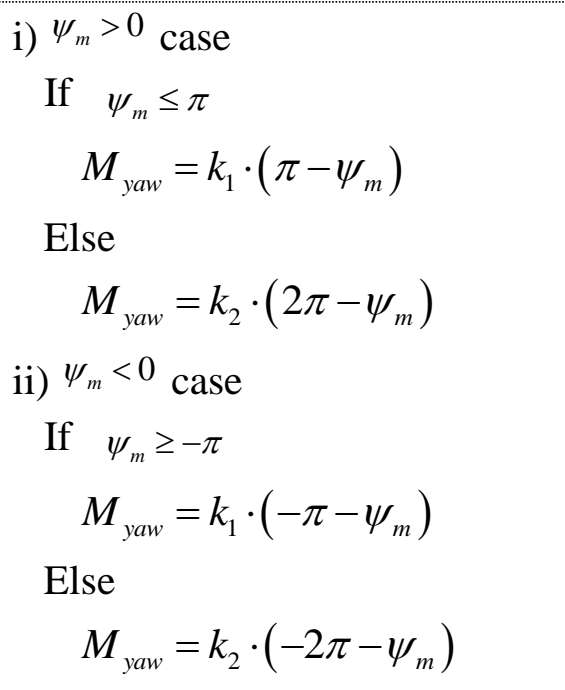 & $\begin{array}{l}\text { Calculate corrective yaw } \\
\text { moment for the yaw angle } \\
\text { regulation } \\
\left(k_{1}, k_{2}: \text { control gains) }\right.\end{array}$ \\
\hline (4) No Control & $F_{x, i}=0 \quad\left(i=f_{L}, f_{R}, r_{L}, r_{R}\right)$ & Free wheel rolling \\
\hline $\begin{array}{l}\text { (5) Stabilization } \\
\text { Control }\end{array}$ & 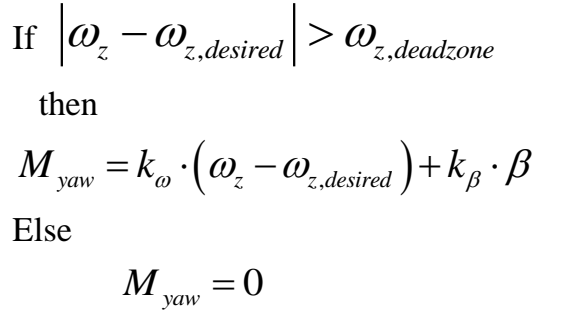 & $\begin{array}{l}\text { Regulate yaw rate and side } \\
\text { slip angle to stabilize } \\
\text { vehicle motion } \\
\left(k_{\omega}, k_{\beta}: \text { control gains) }\right.\end{array}$ \\
\hline
\end{tabular}


Table 5.2 Rule-based mode switching logic

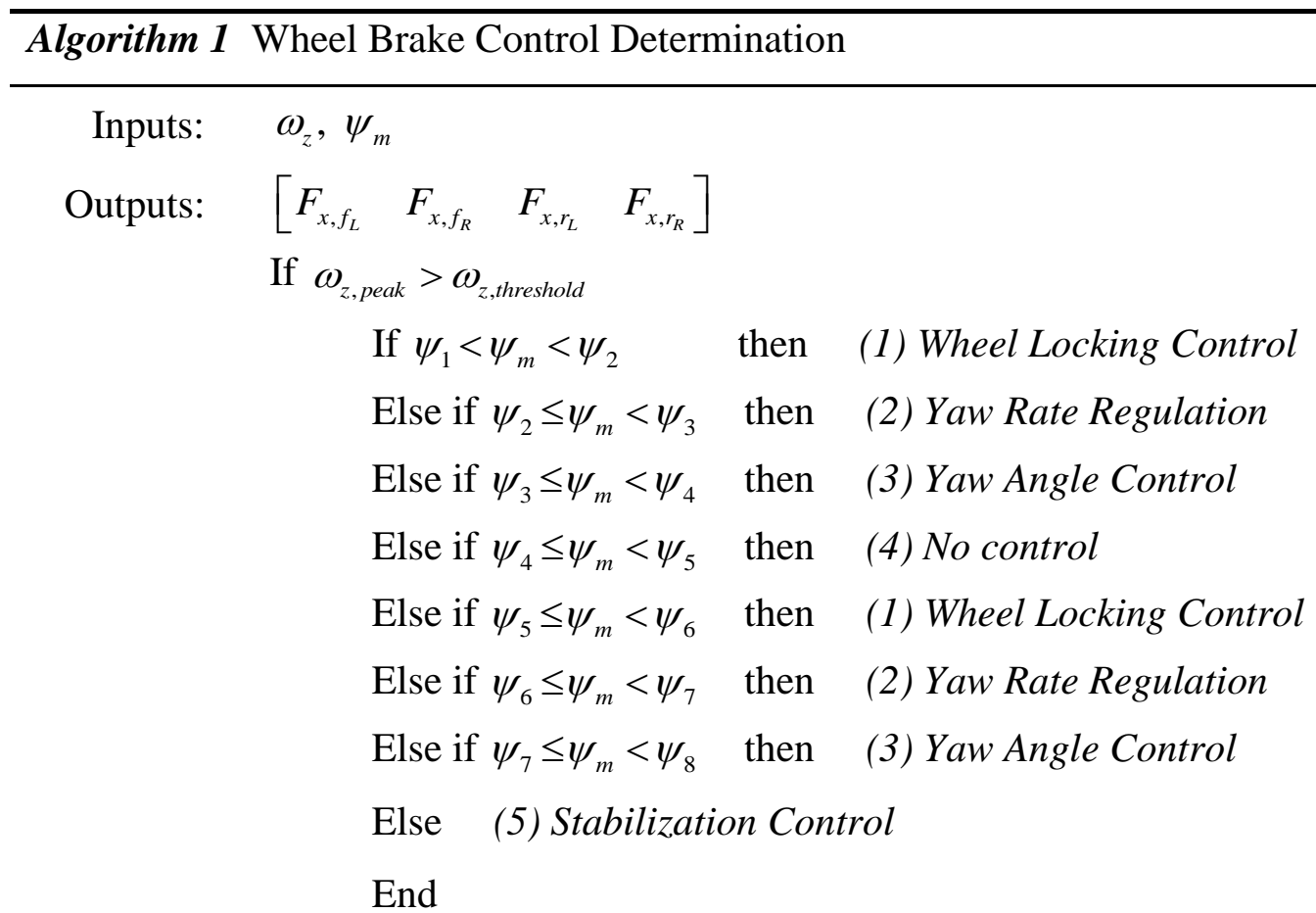

Else if $\omega_{z, \text { peak }}<-\omega_{z, \text { threshold }}$

If $-\psi_{2} \leq \psi_{m} \leq-\psi_{1} \quad$ then (1) Wheel Locking Control

Else if $-\psi_{3} \leq \psi_{m} \leq-\psi_{2}$ then (2) Yaw Rate Regulation

Else if $-\psi_{4} \leq \psi_{m} \leq-\psi_{3}$ then (3) Yaw Angle Control

Else if $-\psi_{5} \leq \psi_{m} \leq-\psi_{4}$ then (4) No control

Else if $-\psi_{6} \leq \psi_{m} \leq-\psi_{5}$ then (1) Wheel Locking Control

Else if $-\psi_{7} \leq \psi_{m} \leq-\psi_{6}$ then (2) Yaw Rate Regulation

Else if $-\psi_{8} \leq \psi_{m} \leq-\psi_{7}$ then (3) Yaw Angle Control

Else (5) Stabilization Control

End

Else

(5) Stabilization Control

End 
Table 5.3 Parameters for the switching rule

\begin{tabular}{|c|c|c|c|}
\hline Parameters & Values & Parameters & Values \\
\hline$\psi_{1}$ & $10^{\circ}$ & $\psi_{5}$ & $190^{\circ}$ \\
\hline$\psi_{2}$ & $25^{\circ}$ & $\psi_{6}$ & $200^{\circ}$ \\
\hline$\psi_{3}$ & $90^{\circ}$ & $\psi_{7}$ & $270^{\circ}$ \\
\hline$\psi_{4}$ & $170^{\circ}$ & $\psi_{8}$ & $350^{\circ}$ \\
\hline$\omega_{z, \text { threshold }}$ & $55^{\circ} / \mathrm{sec}$ & - & - \\
\hline
\end{tabular}

Parameters for mode switching rules are presented in

Table 5.3. The yaw rate threshold value $\left(\omega_{z, \text { threshold }}\right)$ is defined based on the analysis in Section 4.5, which describes the final heading angle for small lateral deviation. This means that achieving $180^{\circ}$ heading angle from this yaw rate condition results in a similar lateral deviation level of a $0^{\circ}$ heading angle control.

\subsubsection{Simulation Results}

The proposed rule-based control is evaluated by comparing it to several optimal control results in Section 5.6. The same vehicle model (a big SUV in CarSim) is used for the simulations. Before impact, the vehicle is assumed to travel at $30 \mathrm{~m} / \mathrm{s}(67 \mathrm{mph}, 108$ $\mathrm{kph}$ ) on a flat and straight road. The same settings in Figure 5.12 are applied as initial collision effects: lateral speed $5.0 \mathrm{~m} / \mathrm{s}$, initial yaw rate ranges are $80 \sim 120^{\circ} / \mathrm{s}$ and heading angle: ranges are $9 \sim 17^{\circ}$.

Comparison results are shown in Figure 5.18. Each dot on the plot represents the ratio of settling time and maximum lateral deviation between rule-based control and LTV-MPC at each simulation condition. The criteria to determine the settling time are vehicle yaw rate $\left|\omega_{z}\right|<10^{\circ} / s$ and lateral acceleration $\left|A_{y}\right|<0.1 \mathrm{~g}$. These indicate how much the two control performances are different in terms of settling time and maximum lateral displacement. 


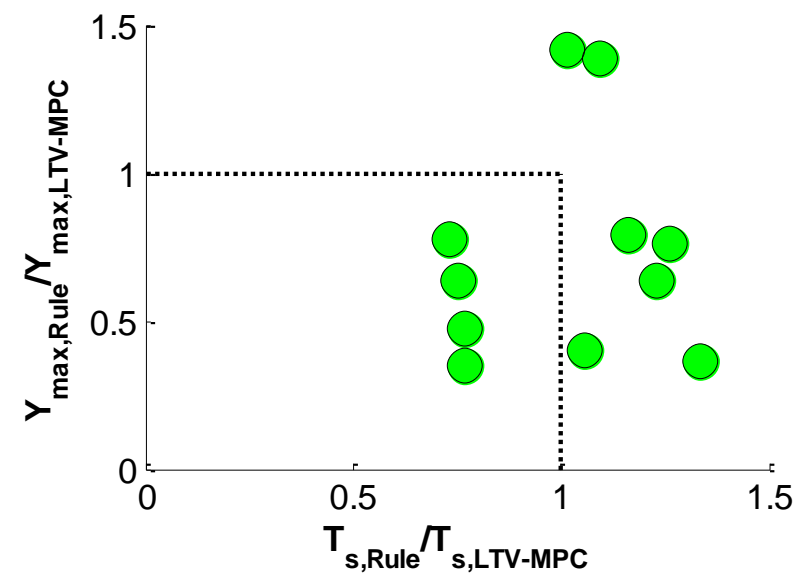

Figure 5.18 Simulation result comparison between the rule-based control and LTV-MPC

Considering that the dotted lines in Figure 5.18 indicate the same performance, the result shows that the settling times vary by about $\pm 30 \%$, and the maximum yaw rates differ by $\pm 60 \%$.

Further evaluation of the rule-based controller is conducted by comparing it to the PISC design developed in [5], where the control aims to regulate both yaw rate and sideslip angle and bring the vehicle back to its original heading - named as 'PISC-toorigin' in results. A series of scenarios is set by increasing the lateral impact force from the reference condition in Table 5.4. Because the initial yaw rate and heading angle are mostly affected by the lateral impact force, variations of the lateral impact forces are determined by the scaling factors as shown in Table 5.5.

Table 5.4 Reference conditions for collision forces

\begin{tabular}{|c|c|c|}
\hline Parameters & Description & Values \\
\hline$F_{x, \max }$ & $\begin{array}{c}\text { X-directional } \\
\text { maximum impact force }\end{array}$ & $8.4673 \mathrm{e}+004[\mathrm{~N}]$ \\
\hline$F_{y, \max }$ & $\begin{array}{c}\text { y-directional } \\
\text { maximum impact force }\end{array}$ & $-3.9484 \mathrm{e}+004[\mathrm{~N}]$ \\
\hline$\Delta T$ & $\begin{array}{c}\text { Collision time duration } \\
\text { (both x- and y- direction) }\end{array}$ & 0.15 seconds \\
\hline$x_{A}$ & $\begin{array}{c}\text { Impact location of the } \\
\text { controlled car (x-direction) }\end{array}$ & $-2.65 \mathrm{~m}$ \\
\hline$y_{A}$ & $\begin{array}{c}\text { Impact location of the } \\
\text { controlled car (y-direction) }\end{array}$ & $0.88 \mathrm{~m}$ \\
\hline$z_{A}$ & $\begin{array}{c}\text { Impact location of the } \\
\text { controlled car (z-direction) }\end{array}$ & $0.65 \mathrm{~m}$ \\
\hline
\end{tabular}


Table 5.5 Series of test conditions

\begin{tabular}{|c|c|c||c|c|c|}
\hline $\begin{array}{c}\text { Test } \\
\text { Cases }\end{array}$ & Factors & $F_{y, \max }$ Values & $\begin{array}{c}\text { Test } \\
\text { Cases }\end{array}$ & Factors & $F_{y, \max }$ Values \\
\hline 1 & 0.80 & $-3.15872 \mathrm{e}+004[\mathrm{~N}]$ & 10 & 1.25 & $-4.9355 \mathrm{e}+004[\mathrm{~N}]$ \\
\hline 2 & 0.85 & $-3.36561 \mathrm{e}+004[\mathrm{~N}]$ & 11 & 1.30 & $-5.1329 \mathrm{e}+004[\mathrm{~N}]$ \\
\hline 3 & 0.90 & $-3.35535 \mathrm{e}+004[\mathrm{~N}]$ & 12 & 1.35 & $-5.3303 \mathrm{e}+004[\mathrm{~N}]$ \\
\hline 4 & 0.95 & $-3.7509 \mathrm{e}+004[\mathrm{~N}]$ & 13 & 1.40 & $-5.5277 \mathrm{e}+004[\mathrm{~N}]$ \\
\hline $\mathbf{5}$ & $\mathbf{1 . 0 0}$ & $-\mathbf{- 3 . 9 4 8 4} \mathrm{e}+\mathbf{0 0 4}[\mathrm{N}]$ & 14 & 1.45 & $-5.7251 \mathrm{e}+004[\mathrm{~N}]$ \\
\hline 6 & 1.05 & $-4.1458 \mathrm{e}+004[\mathrm{~N}]$ & 15 & 1.50 & $-5.9226 \mathrm{e}+004[\mathrm{~N}]$ \\
\hline 7 & 1.10 & $-4.3432 \mathrm{e}+004[\mathrm{~N}]$ & 16 & 1.55 & $-6.1200 \mathrm{e}+004[\mathrm{~N}]$ \\
\hline 8 & 1.15 & $-4.5406 \mathrm{e}+004[\mathrm{~N}]$ & 17 & 1.60 & $-6.3174 \mathrm{e}+004[\mathrm{~N}]$ \\
\hline 9 & 1.20 & $-4.7380 \mathrm{e}+004[\mathrm{~N}]$ & - & - & - \\
\hline
\end{tabular}

Here, 17 cases are tested to compare the control performance. Figure 5.19 shows the initial conditions when controls are initiated. It is obvious that a higher lateral impact force induces higher yaw rate and lateral speed.
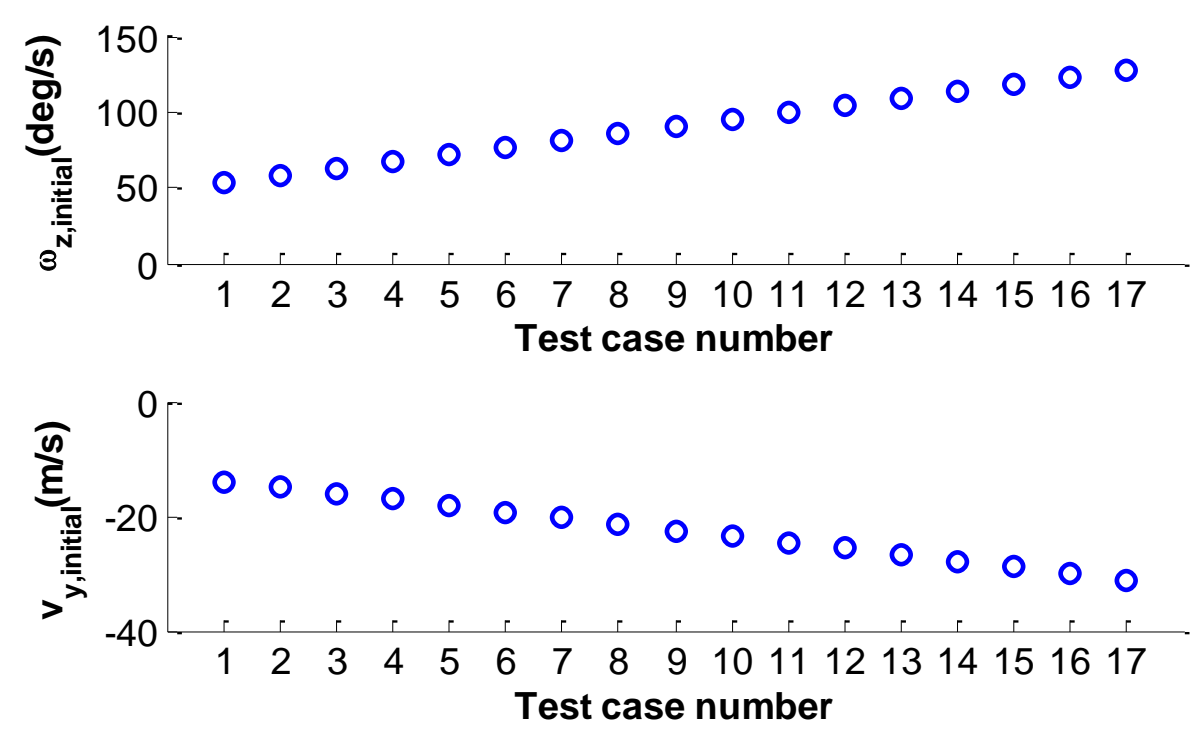

Figure 5.19 Vehicle dynamics parameters when control actions initiated 


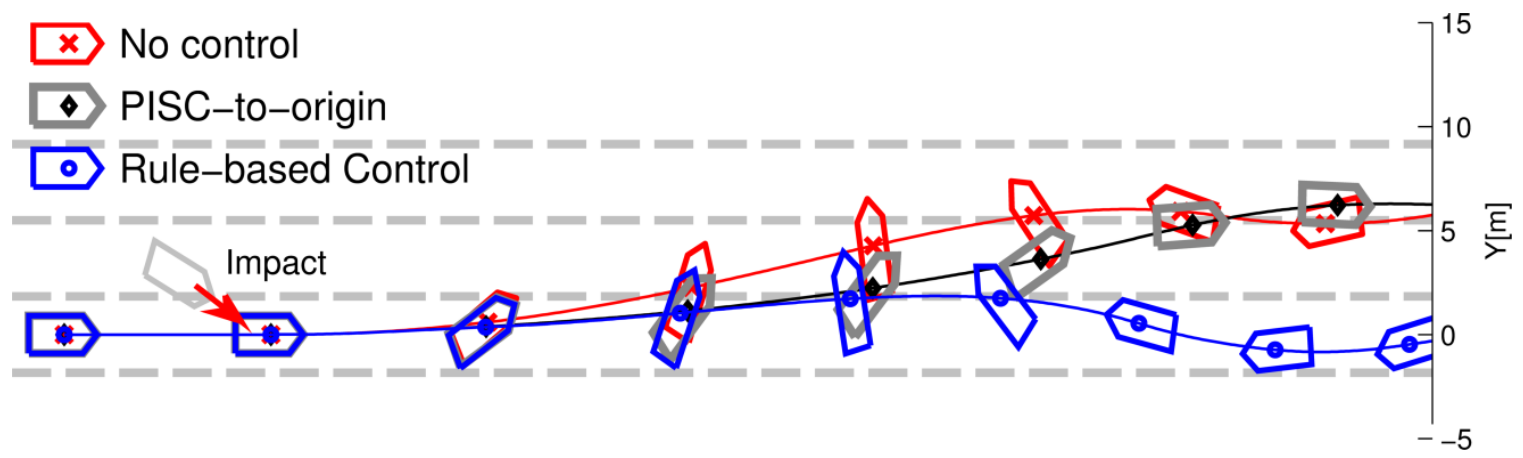

(a) Test case \#5

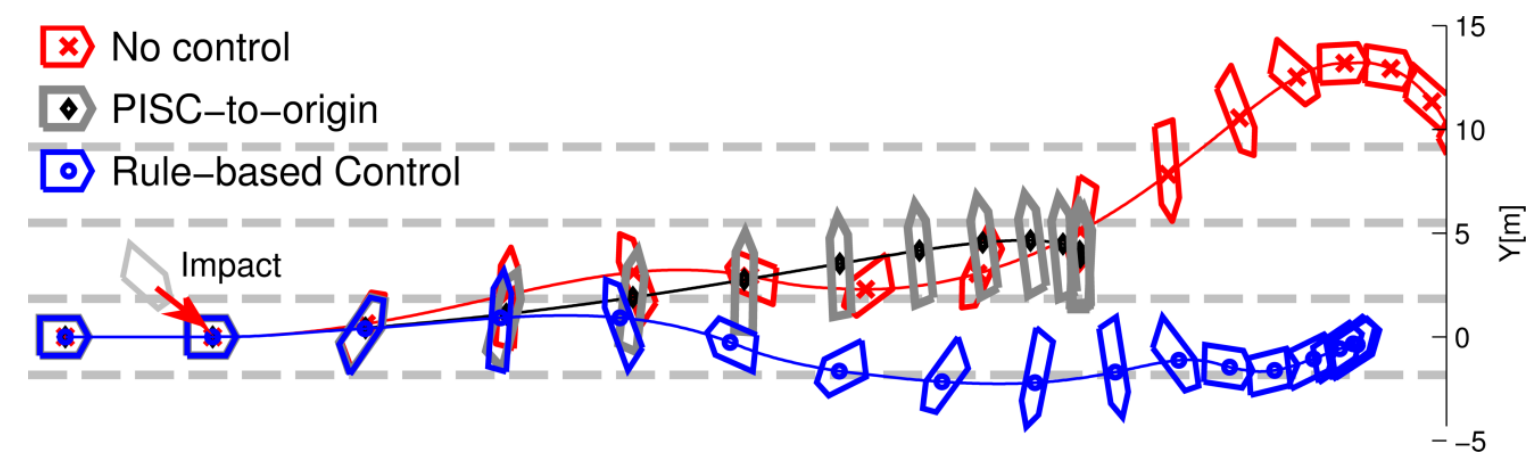

(b) Test case \#9

Figure 5.20 Vehicle trajectory comparisons

Comparisons of the vehicle trajectories for two of the 17 cases are shown in Figure 5.20. Vehicle trajectories without control (red cars) have large lateral deviation with arbitrary heading angles. Although the controller in gray cars ('PISC-to-origin') try to bring the vehicle back to the origin, the lateral deviation becomes high and the vehicle in Test case $\# 9$ even lands at $90^{\circ}$ heading angle. The trajectories with the rule-based control (blue cars) in general have much smaller lateral displacement and better final heading angle.

Control performances in all 17 cases are compared in Figure 5.21. The maximum lateral deviation and the settling time are used as the key performance indices. It is noted that rule-based controller consistently shows smaller lateral deviation. Although there are some cases (Test case \#14, 15, 17) where the 'PISC-to-origin' shows smaller deviations than the rule-based ones, they happen by chance when the vehicle turns to a $180^{\circ}$ or $360^{\circ}$ heading angle. In contrast, the settling time $\left(T_{s}\right)$ differences between the two controllers 
are small. The settling time criteria are vehicle yaw rate $\left|\omega_{z}\right|<10^{\circ} / \mathrm{s}$ and lateral acceleration $\left|A_{y}\right|<0.1 \mathrm{~g}$, the same as the settings for Figure 5.18. The rule-based control in Test case numbers 1-5 manipulate the vehicle to land at $180^{\circ}$ heading angle and control to $360^{\circ}$ heading angle in the rest of the cases. Thus, these simulation results demonstrate that the proposed control strategy achieves significant reduction in lateral deviation compared with the PISC control.
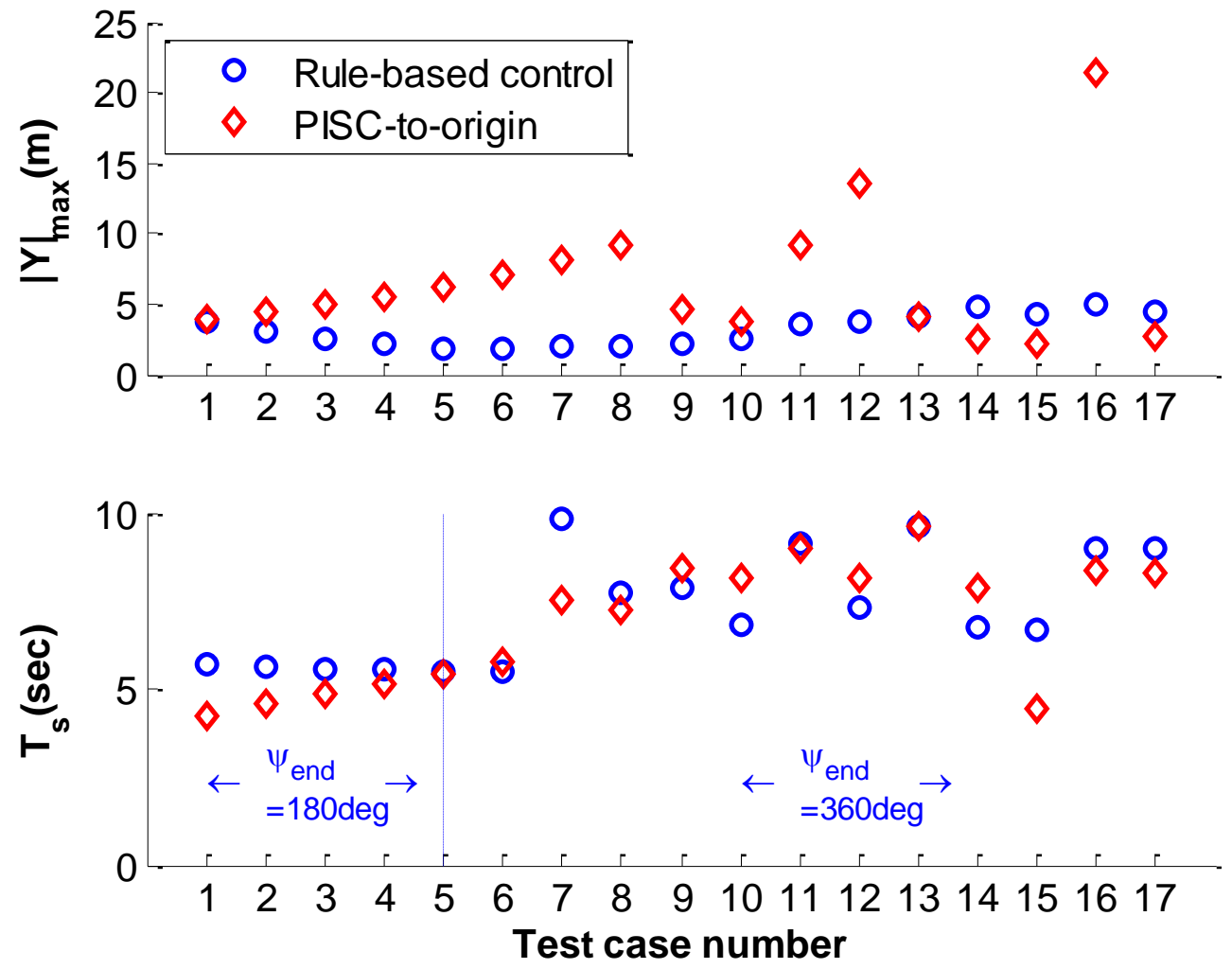

Figure 5.21 Simulation results of 17 different cases 


\subsection{Steering for Control Enhancement}

Active steering is an effective way to control a vehicle's yaw and roll dynamics as shown in [86]. For example, as illustrated in Figure 5.22, the amount of yaw moment that can be generated by steering is compared to the available yaw moment with left twowheel braking. The available yaw moment torque from the left two-wheel braking is:

$$
M_{\text {brake }}=\left(F_{f, \max }+F_{r, \text { max }}\right) \cdot T_{W} / 2
$$

The one from front wheel steering is (assuming that the nominal condition of the vehicle is driving straight with no steering):

$$
M_{\text {steer }}=2 \cdot F_{f, \max } \cdot a
$$

Typically, the distance between the front axle distance to the CG $(a)$ is longer than the half of track width $\left(T_{W} / 2\right)$. For a front-wheel drive vehicle, the maximum force

on the front tires $\left(F_{f, \text { max }}\right)$ is larger than the rear tires $\left(F_{r, \text { max }}\right)$ because the normal loads on front tires are higher than the rear, thus in turn $M_{\text {brake }}<M_{\text {steer }}$. For this reason, steering can generate higher yaw moment than differential braking.

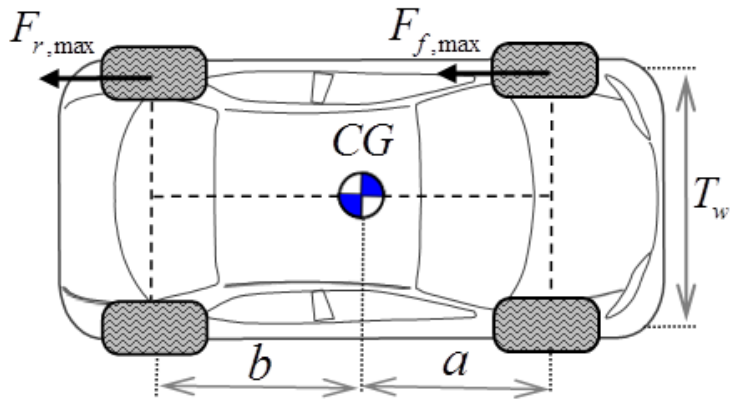

(a) Differential braking case

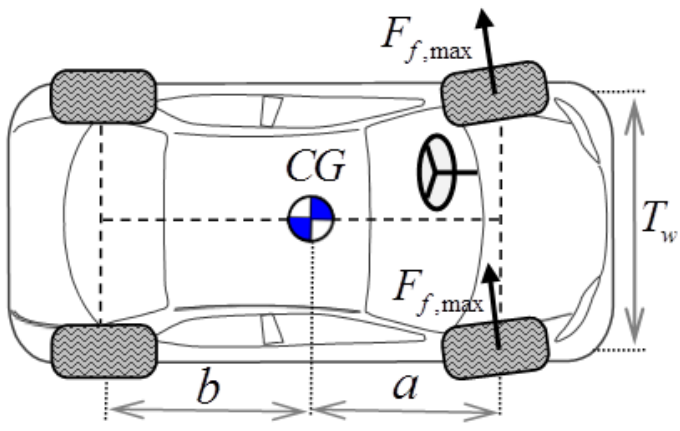

(b) Front wheel steering case

Figure 5.22 Generating yaw moment by using differential braking and steering

Furthermore, the steering control can create different slip angles between the front and rear tires, which in turn can generate a yaw moment of the vehicle. Thus, even in a 
higher side slip angle situation where brake controls are limited, steering action expands the range of yaw moment control. For example, imagine that the vehicle has a positive yaw rate and all tires initially have negative slip angles after an impact, shown as circles in Figure 5.23 (b). The possible yaw moment from wheel brake control actions are calculated from the kinematic relationships shown in Equations (5.45)-(5.48) and plotted as solid and dashed lines in Figure 5.23.

$$
\begin{aligned}
& M_{z, f_{L}}=F_{x, f_{L}} \cdot\left(a \cdot \sin (\delta)-\frac{T_{W}}{2} \cdot \cos (\delta)\right)+F_{y, f_{L}} \cdot\left(a \cdot \cos (\delta)+\frac{T_{W}}{2} \cdot \sin (\delta)\right) \\
& M_{z, f_{R}}=F_{x, f_{R}} \cdot\left(a \cdot \sin (\delta)+\frac{T_{W}}{2} \cdot \cos (\delta)\right)+F_{y, f_{R}} \cdot\left(a \cdot \cos (\delta)-\frac{T_{W}}{2} \cdot \sin (\delta)\right) \\
& M_{z, r_{L}}=-F_{x, r_{L}} \cdot \frac{T_{W}}{2}-F_{y, r_{L}} \cdot b \\
& M_{z, r_{R}}=F_{x, r_{R}} \cdot \frac{T_{W}}{2}-F_{y, r_{R}} \cdot b
\end{aligned}
$$

The vehicle in Figure 5.23 (a) needs a clockwise yaw moment about the CG (negative yaw moment). When all tires have the same negative sign in slip angles, the rear tires naturally produce negative yaw moment even without brake action. The front right tire can help to produce the negative yaw moment when brake is applied. Due to the coupling effect between longitudinal and lateral tire forces, braking on the rear right wheel does not help at all to increase the clockwise yaw moment.

On the other hand, if steering action is involved, it can change the slip angles of the front tires so that both front tires can generate the needed yaw moment, illustrated as 'stars' in Figure 5.23 (b). As long as the slip angles on the front tires are within the range of the steering angle limit, steering can provide more control authority than brake control only. In this sense, a proper steering control action can expand the region of control authority. 


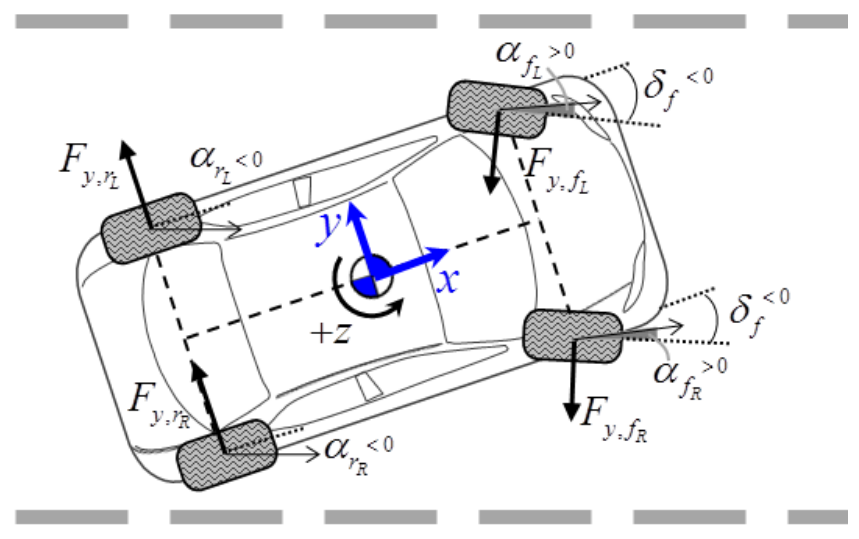

(a)

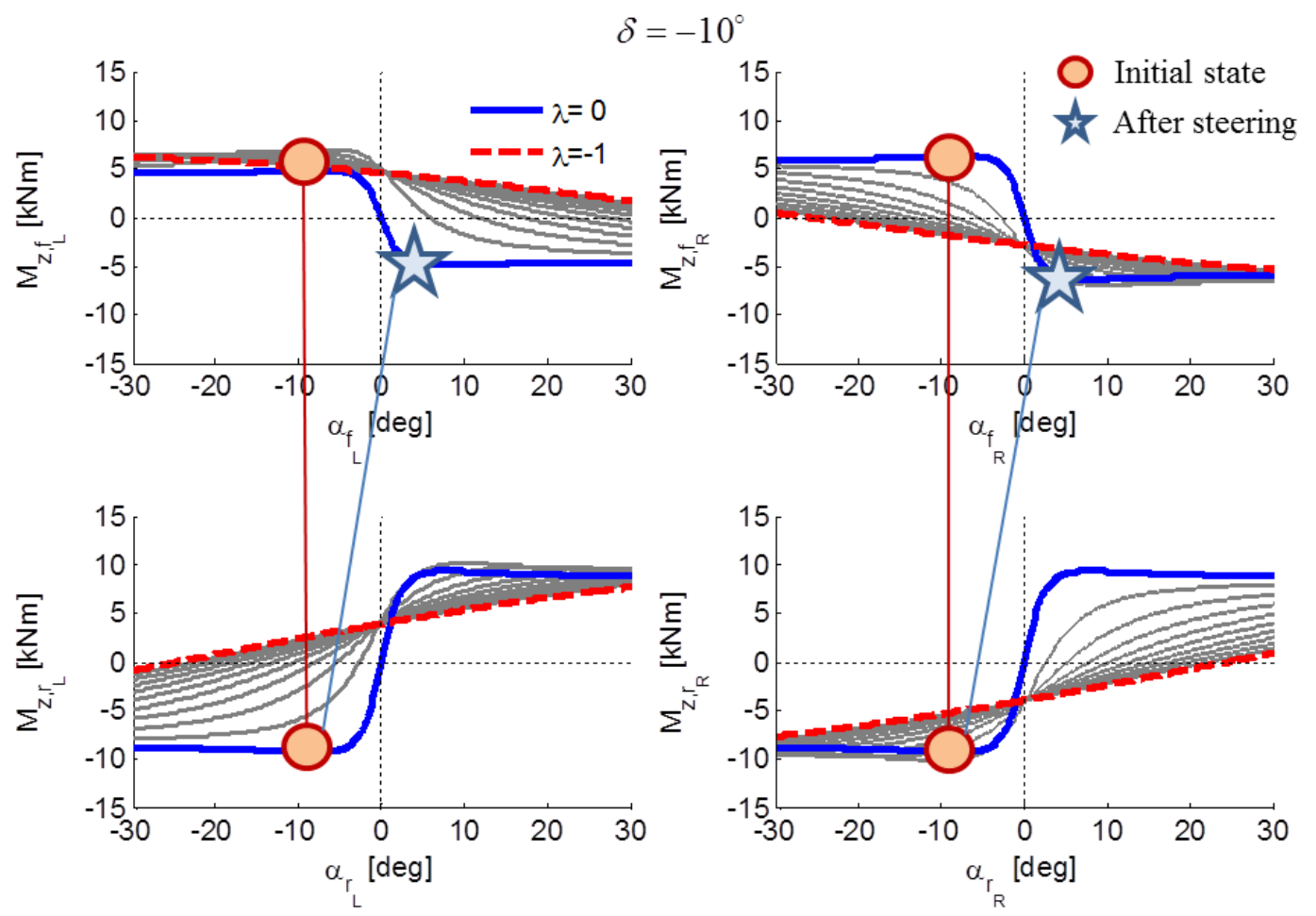

(b)

Figure 5.23 Vehicle dynamics showing the steering changes the direction of the front tire forces and yaw moment to the vehicle CG. (a) Vehicle model showing the tire force, slip angle, and steering angle. (b) Possible yaw moments that can be generated by each tire. Red circles indicate $0^{\circ}$ steering, the blue stars indicate $-10^{\circ}$ steering. Gray lines are yaw moment with a tire slip ratio between $\lambda=0$ (no braking) and $\lambda=-1$ (wheel locking). 


\subsubsection{Steering Action Analysis}

Although steering action is an effective mechanism to control vehicle yaw motion, it has a limited availability to generate yaw moments as tire forces saturate due to the friction limit. If the vehicle has a high slip angle before control actions are applied, the steering action may not change the lateral tire forces effectively. Moreover, the yaw moment achieved by steering starts to saturate as lateral tire forces enter the nonlinear region. Figure 5.24 shows the open loop dynamics on the $\beta-\omega_{z}$ phase plane at $30 \mathrm{~m} / \mathrm{s}$ (67 mph, $108 \mathrm{kph}$ ) for several steering angles. The same equations and settings, illustrated in Figure 4.1, are used to obtain the phase plots. Note that the maximum amount of yaw rate by steering (depicted in black bold lines) does not linearly increase with the steering angle. The peak yaw rate levels in (d), (e), and (f) are similar ( $\omega_{z}$ are approximately $-20^{\circ} / \mathrm{s}$ ), even though the steering angle increases from $-5^{\circ}$ to $-15^{\circ}$. This result indicates that the peak yaw moment by steering saturates due to tire-road friction limit. In other words, the maximum yaw rate is also limited. In addition, the size of the spiral shaped trajectory around the equilibrium point increases as the steering angles are high, meaning that the yaw rate response seems to become oscillatory in high steering angles.

The maximum yaw rate amount also changes with the vehicle speed, as shown in Figure 5.25. The peak yaw rates increase from (a) to (c), while the peak yaw rates are saturated in (d), (e), and (f). In the lower speed cases, such as (a), (b), and (c), the equilibrium points are located in the region where slip angle values have negative signs, while the equilibrium points at higher speeds $\left(v_{x} \geq 20 \mathrm{~m} / \mathrm{s}\right)$ appear at positive side slip angle. This is a well-known vehicle dynamics effect, "under-steer". As most front-wheeldrive vehicles, like the vehicle model in this simulation, have a tendency to under-steer, this happens when the front tires approach to the friction limit and the lateral forces from those tires do not increase any more. 


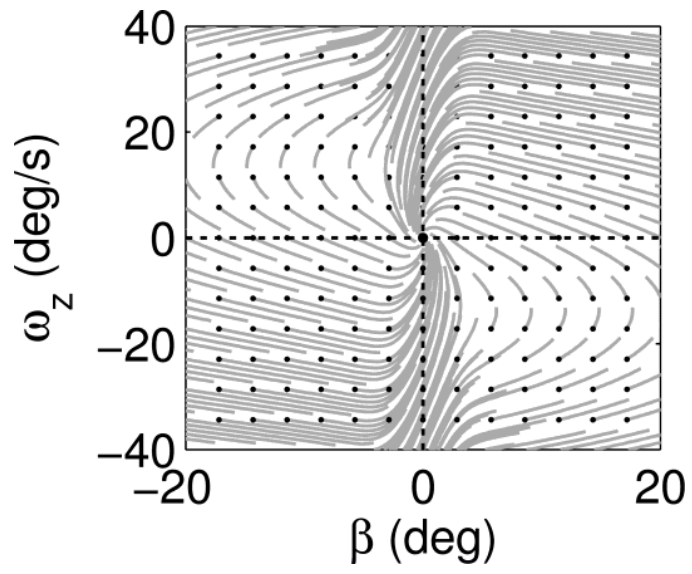

(a) $\delta=0^{\circ}$

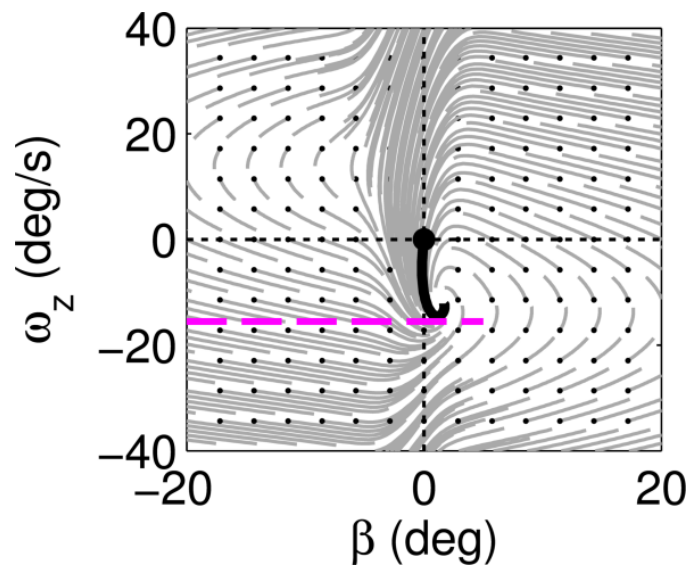

(c) $\delta=-2^{\circ}$

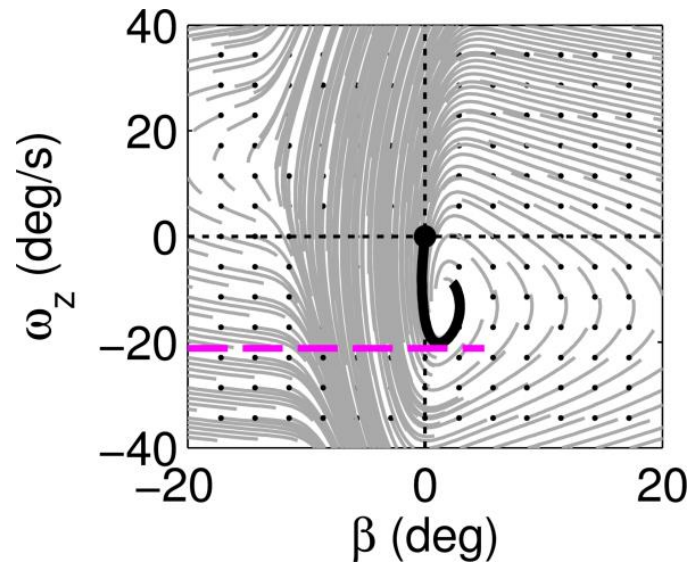

(e) $\delta=-10^{\circ}$

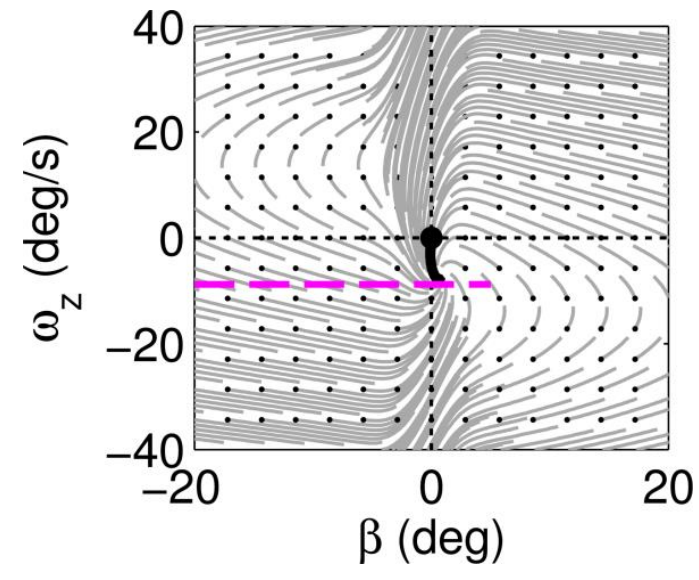

(b) $\delta=-1^{\circ}$

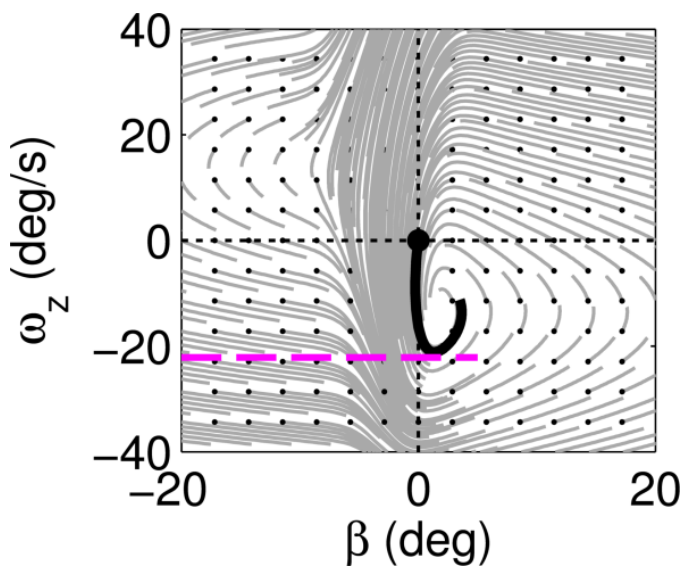

(d) $\delta=-5^{\circ}$

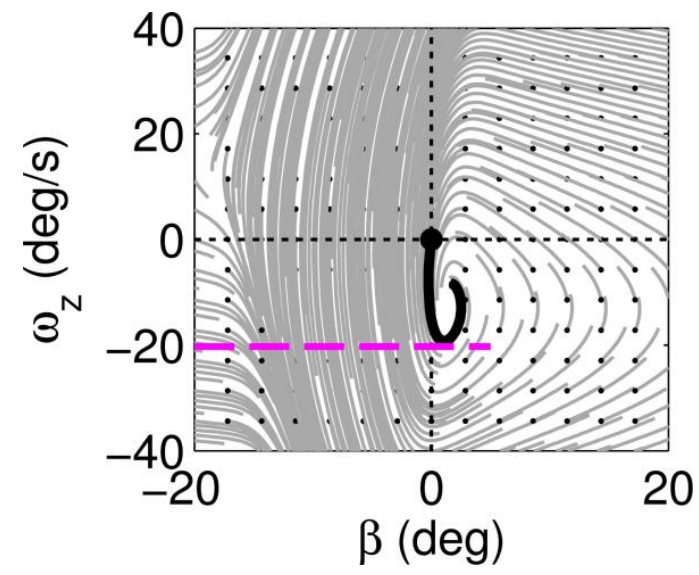

(f) $\delta=-15^{\circ}$

Figure 5.24 Open loop dynamics on the phase plane at $v_{x}=30 \mathrm{~m} / \mathrm{s}$ with different steering angles (Black bold lines: vehicle trajectories from the origin for 1 second, magenta dashed line indicates peak level of yaw rate from the origin) 


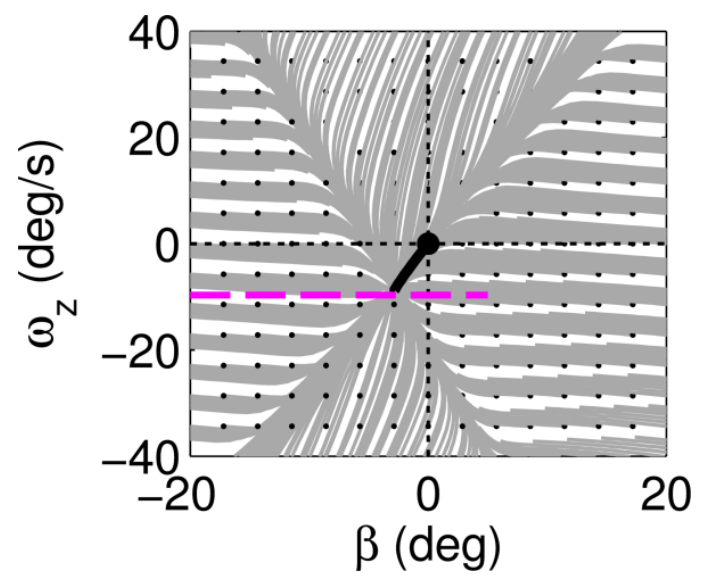

(a) $v_{x}=5 \mathrm{~m} / \mathrm{s}$

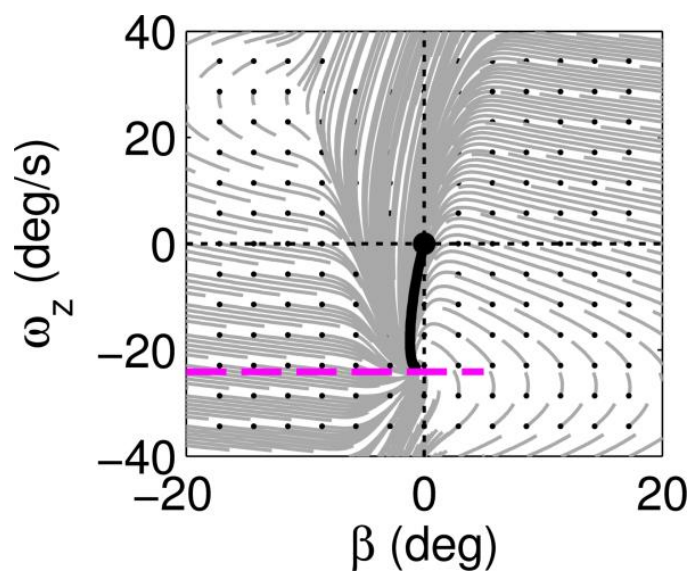

(c) $v_{x}=15 \mathrm{~m} / \mathrm{s}$

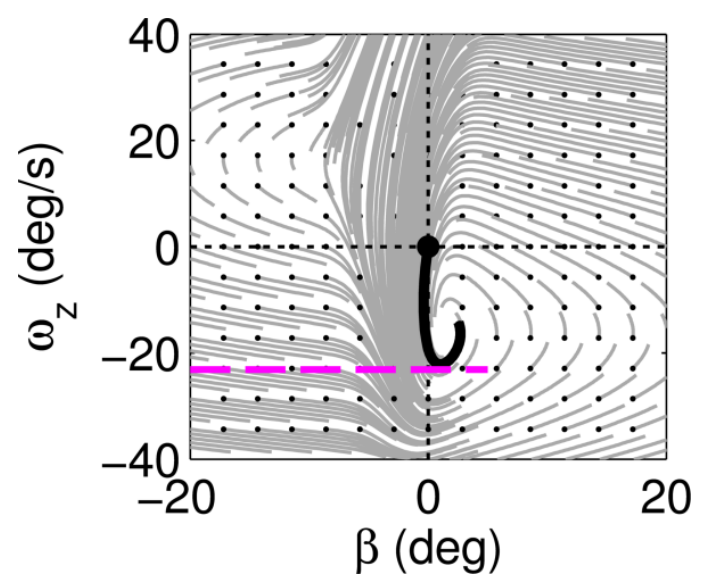

(e) $v_{x}=25 \mathrm{~m} / \mathrm{s}$

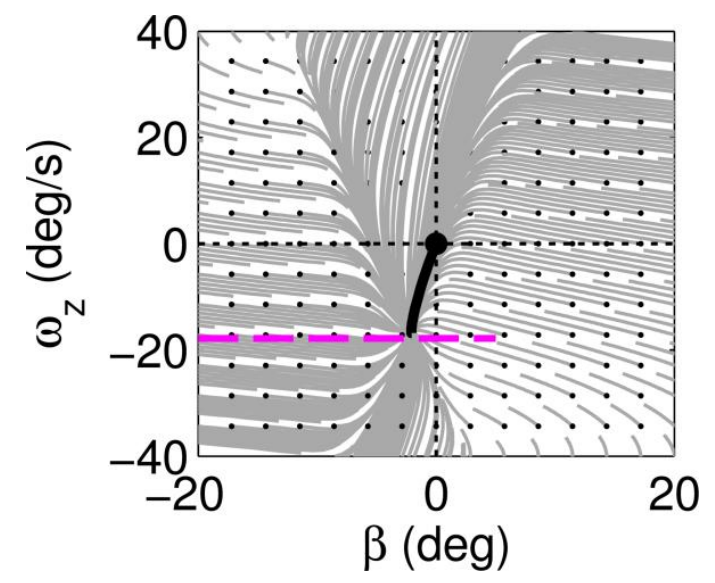

(b) $v_{x}=10 \mathrm{~m} / \mathrm{s}$

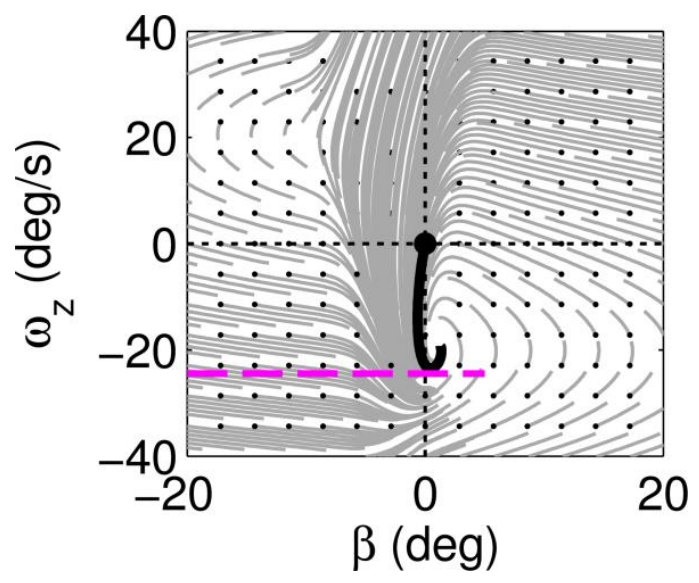

(d) $v_{x}=20 \mathrm{~m} / \mathrm{s}$

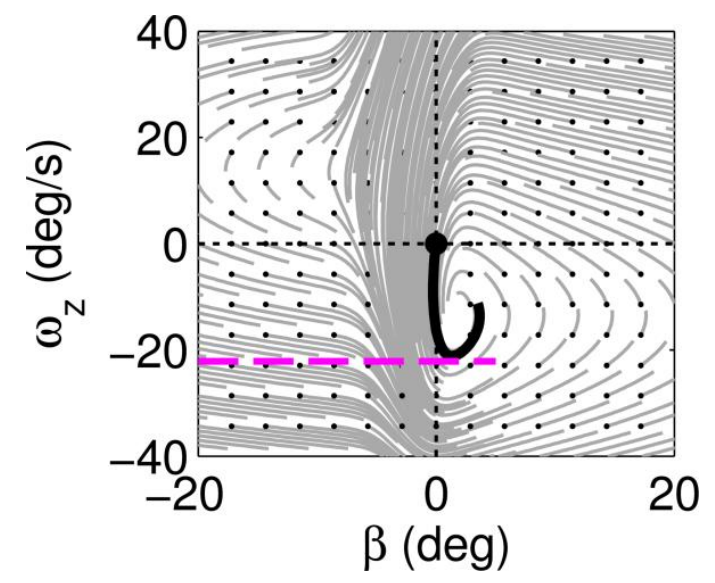

(f) $v_{x}=30 \mathrm{~m} / \mathrm{s}$

Figure 5.25 Open loop dynamics on the phase plane with $\delta=-5^{\circ}$ at different vehicle speed conditions (Black bold lines: vehicle trajectories from the origin for 1 second, magenta dashed line indicates peak level of yaw rate) 
Simulation results in Figure 5.26 also show vehicle yaw rate saturation at high vehicle forward speeds. The simulations were performed with the same fixed steering angle input but at different initial vehicle speeds in CarSim. The results show that the peak yaw rate is achieved at $60 \mathrm{kph}$ speed and may decrease continuously passed the peak.

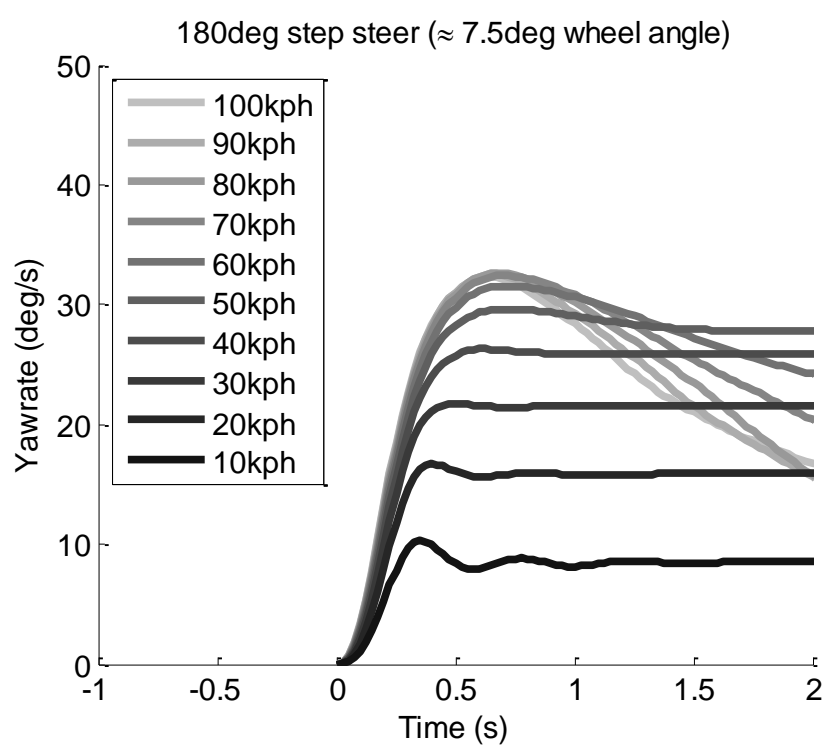

Figure 5.26 Yaw rate responses with a same step steering input under different longitudinal speeds

Based on the above results, we could conclude that the steering control for vehicle yaw moment regulation is a challenging task when the front tires are saturated. This is especially true at high forward speeds. In other words, the effectiveness of the steering control is attenuated if the tires are already saturated. Therefore, prompt control initiation before tire saturation is beneficial. By doing so, yaw rate and side slip angle are suppressed effectively and subsequent differential braking control performance can be enhanced.

\subsubsection{Preemptive Steering Control}

To maximize the benefit of the active steering control, preemptive steering control might be a possible solution. Suppose that the system can trace other vehicle moving trajectories and detect approaching vehicles, as shown in Figure 5.27. Then, a counter- 
yaw moment generated before the collision can significantly reduce the yaw moment from the collision. A major benefit of this action is to avoid large tire slip angles, which render differential braking ineffective [87].

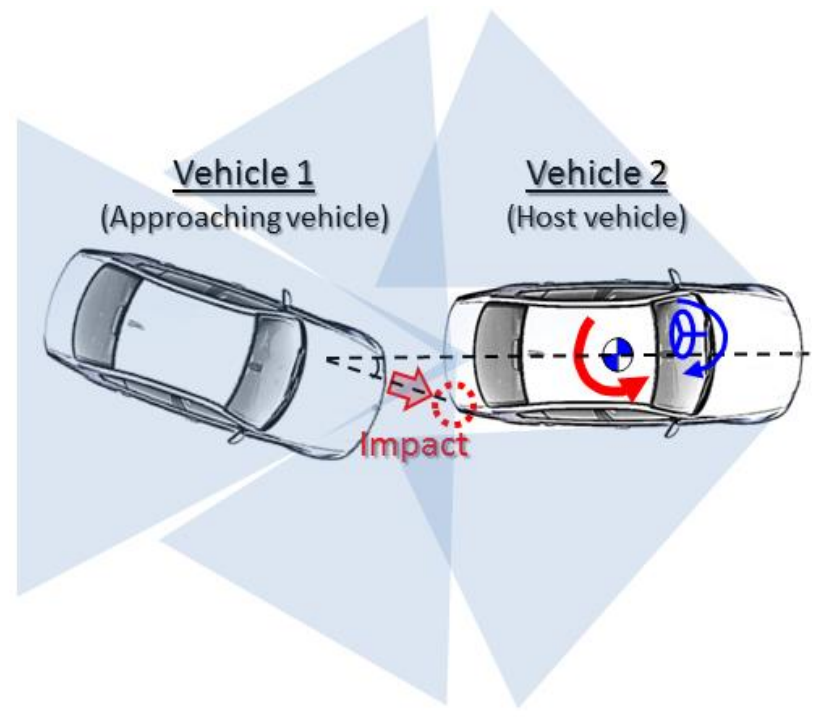

Figure 5.27 A crash scenario showing a counter-steering control to negate the vehicle motion due to an impact (Shaded triangle regions illustrate possible sensor coverage for detecting an approaching vehicle to the host vehicle)

The steering action changes the vehicle lateral and yaw motion. As seen in the previous analysis shown in Figure 5.24, the equilibrium point with a nonzero steering angle is not the origin. So, the resulting peak yaw rate due to an impact can be reduced because the yaw rate in the opposite direction is developed in advance by the steering action. In addition, this steering action mitigates large sideslip angle development and yaw rate divergence after an impact. Because of the preemptive control, as shown in Figure 5.28 (a) and (b), faster sideslip reduction and yaw rate convergence can be achieved. The red dot in (a) represents the resulting yaw rate and sideslip angle after an initial impact and the red dashed line from the dot is the vehicle motion as they evolve over time. The black solid line in (b) is the traces of vehicle motion from the origin induced by the steering action before the impact. In this case, the vehicle state in blue dot caused by the impact shows smaller magnitude of yaw rate and sideslip angle than the red dot in (a). In addition, the vehicle motion trace in blue dashed line exhibits a fast converging tendency to the equilibrium. 


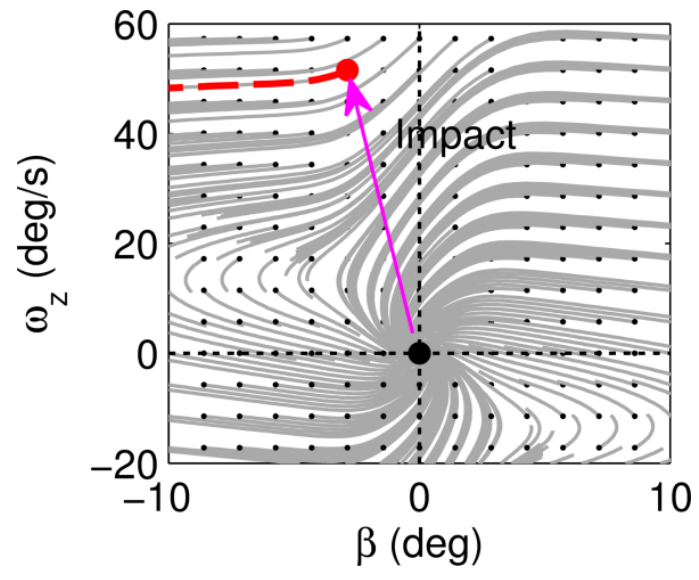

(a) $\delta=0^{\circ}$

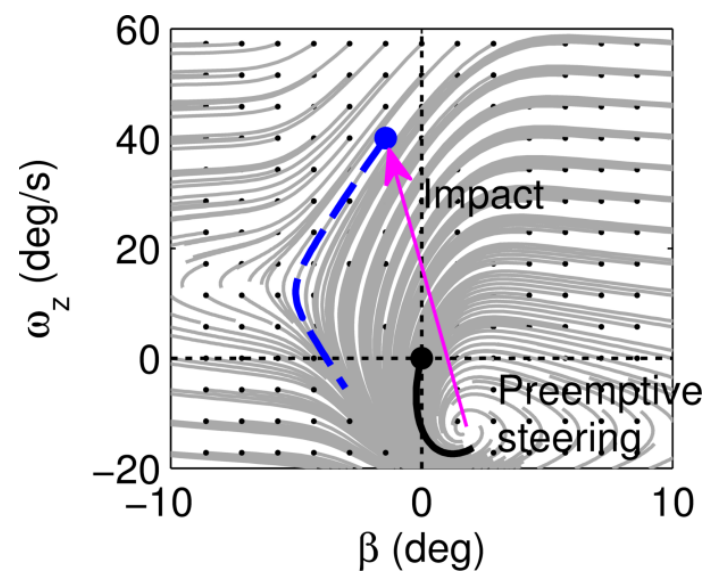

(b) $\delta=-2.5^{\circ}$

Figure 5.28 Vehicle motions on the phase plane at $v_{x}=30 \mathrm{~m} / \mathrm{s}$ showing the advantage of preemptive steering action. The vehicle motion with an impact condition without any steering action, which causes $\Delta \omega_{z}=50^{\circ} / s, \Delta \beta=-3^{\circ}$, is shown in (a), and the vehicle motion with a preemptive steering $\left(-2.5^{\circ}\right)$ prior to the same impact is shown in (b)

The designed system monitors vehicles in adjacent lanes and behind the host vehicle. When the motion of a vehicle around the host vehicle is detected as a hazard for imminent collision, the system applies a required steering angle to generate counter-yaw moment against the collision force. The impact position and angle are assumed to be available from sensors. The force prediction and estimation in Chapter 2 play major roles in determining the feed-forward control input. While significant vehicle motion may still develop during and after an impact, a feedback controller starts to take action to attenuate the vehicle motions not cancelled by the feed-forward action. As shown in Figure 5.29, the final desired steering commands is the sum of feed-forward and feedback control actions.

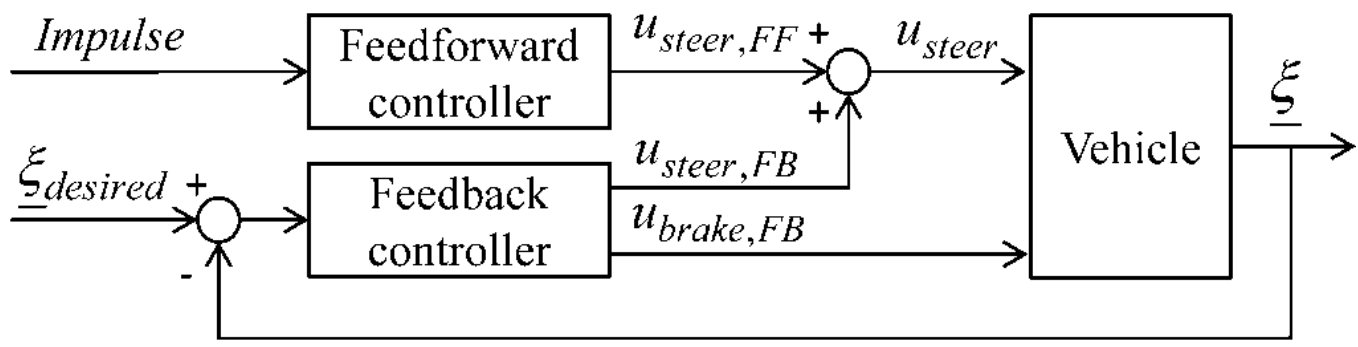

Figure 5.29 Block diagram of the control structure, $\underline{\xi}=\left[\begin{array}{ll}v_{y} & \omega_{z}\end{array}\right]^{T}$ 


\subsubsection{Feed-forward Control}

The purpose of the feed-forward control is to achieve faster vehicle motion response to alleviate the collision effect preemptively. The steering angle is determined by the expected vehicle motion after the impact. As shown in Figure 5.30, the first step is to estimate the collision force. Because the control action needs to be initiated before the collision occurs, impulse estimation should be simpler than the one shown in Equations (3.1)-(3.3) as there are no sensor measurements yet. Before the collision happens, the impulses can only be estimated by expected speed differences from the collision:

$$
\begin{gathered}
m_{1} \cdot v_{1}+m_{2} \cdot v_{2}=\left(m_{1}+m_{2}\right) \cdot v_{f} \\
\Rightarrow v_{f}=\frac{m_{1} \cdot v_{1}+m_{2} \cdot v_{2}}{m_{1}+m_{2}}
\end{gathered}
$$

where, the subscripts 1 and 2 represent 'vehicle 1 and 2', and $v_{f}$ is the final speed after collision. By assuming that the masses of the two cars are identical, Equation (10) is simplified to an average speed of both car speeds $\left(v_{f} \approx\left(v_{1}+v_{2}\right) / 2\right)$. By applying this relationship in both $x$ - and $y$-directions, the magnitudes of the final velocity components $\left(v_{f, x}, v_{f, y}\right)$ are found. To calculate the expected impulse strength (the change in momentum before and after the collision), the linear momentum equations are used:

$$
\begin{aligned}
& \Delta \hat{P}_{x}=m \cdot\left(v_{f, x}-v_{x}\right) \\
& \Delta \hat{P}_{y}=m \cdot\left(v_{f, y}-v_{y}\right)
\end{aligned}
$$

Since we only consider the motion of the control vehicle, subscript 1 is omitted for convenience. The "identical mass assumption" is adequate to start the control action. When better information is available, it certainly can be used for a more accurate estimation and control. Once the impulse strength is estimated, the expected collision force profiles can be predicted using the relationship shown in Equation (3.4). 


$$
\hat{F}_{x_{\max }}=2 \cdot \Delta \hat{P}_{x} / \Delta T, \quad \hat{F}_{y_{\max }}=2 \cdot \Delta \hat{P}_{y} / \Delta T
$$

Next, the vehicle motion is predicted by using the vehicle model shown in Equation (2.1)-(2.4). The target yaw motion with the steering control is set to cancel the predicted vehicle motion due to the impact. Obviously, when the collision happens, the impulse estimation and collision force predictions can be conducted in the same way as described in Figure 3.1.

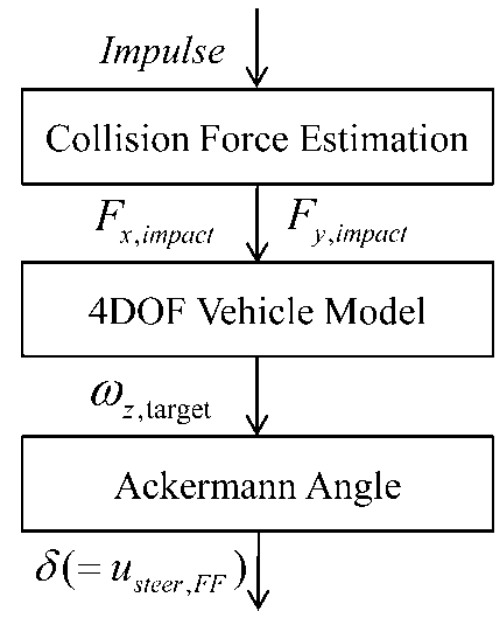

Figure 5.30 Structure for the Feed-forward steering control

The mathematical relationship between the steering angle $(\delta)$ and target vehicle yaw rate $\left(\omega_{z, \text { target }}\right)$ is obtained from the Ackermann angle [88]:

$$
\delta=\left\{\frac{(a+b)}{v_{x}}+K_{u} \cdot v_{x}\right\} \cdot \omega_{z, \text { target }}
$$

where, $K_{u}=-\frac{m}{a+b} \cdot\left\{\frac{a}{C_{\alpha, r}}-\frac{b}{C_{\alpha, f}}\right\}$ is the vehicle under-steer coefficient.

\subsubsection{Feedback Control}

The feedback controller is designed by using the planar 3-DOF vehicle model which excludes the roll motion and external forces in Equations (2.2) and (2.3). The governing equations are: 


$$
\begin{aligned}
& m \cdot\left(\dot{v}_{y}+v_{x} \omega_{z}\right)=\left(F_{x, f_{L}}+F_{x, f_{R}}\right) \sin \delta+\left(F_{y, f_{L}}+F_{y, f_{R}}\right) \cos \delta+\left(F_{y, r_{L}}+F_{y, r_{R}}\right) \\
& I_{z z} \dot{\omega}_{z}=a\left(F_{x, f_{L}}+F_{x, f_{R}}\right) \sin \delta+a\left(F_{y, f_{L}}+F_{y, f_{R}}\right) \cos \delta-b\left(F_{y, r_{L}}+F_{y, r_{R}}\right) \\
& +\frac{T_{W}}{2}\left(F_{x, r_{R}}-F_{x, r_{L}}\right)+\frac{T_{W}}{2}\left[\left(F_{x, f_{R}}-F_{x, f_{L}}\right) \cos \delta-\left(F_{y, f_{R}}-F_{y, f_{L}}\right) \sin \delta\right]
\end{aligned}
$$

To cancel the effect of the disturbance, the sliding mode control concept is applied. Since the purpose of the control is to bring both lateral velocity and yaw rate to the desired states $\left(v_{y, d}, \omega_{z, d}\right)$, the multiple sliding surface control theory [28,89] is applied to suppress those output errors. The first and the second sliding surfaces are defined as

$$
\begin{gathered}
S_{1}=v_{y}-v_{y, d} \\
S_{2}=\omega_{z}-\omega_{z, d}
\end{gathered}
$$

To achieve the control objectives, it is desired that

$$
\begin{aligned}
& \dot{S}_{1}=-k_{1} \cdot S_{1} \\
& \dot{S}_{2}=-k_{2} \cdot S_{2}
\end{aligned}
$$

where, $k_{1}$ and $k_{2}$ are positive definite values that can be chosen for desired convergence rate. Then the sliding surfaces yield the following lateral and yaw accelerations:

$$
\begin{gathered}
\dot{v}_{y}=\dot{v}_{y, d}-k_{1} \cdot\left(v_{y}-v_{y, d}\right) \\
\dot{\omega}_{z}=\dot{\omega}_{z, d}-k_{2} \cdot\left(\omega_{z}-\omega_{z, d}\right)
\end{gathered}
$$

Substituting Equation (5.60) into (5.54) and rearranging yields the desired vehicle yaw rate $\left(\bar{\omega}_{z, d}\right)$ for the first sliding surface:

$$
\bar{\omega}_{z, d}=\frac{\left(\hat{F}_{x, f_{L}}+\hat{F}_{x, f_{R}}\right) \sin \delta+\left(\hat{F}_{y, f_{L}}+\hat{F}_{y, f_{R}}\right) \cos \delta}{m \cdot v_{x}}+\frac{\hat{F}_{y, r_{L}}+\hat{F}_{y, r_{R}}}{m \cdot v_{x}}-\frac{\dot{v}_{y, d}-k\left(v_{y}-v_{y, d}\right)}{v_{x}}
$$


Here, we assume that an estimator for both longitudinal and lateral tire forces exists. The estimated values are marked with the “^” symbol. The yaw rate command in Equation (5.62) is processed with a first-order filter to feed into the second sliding surface.

$$
\tau \cdot \dot{\omega}_{z, d}+\omega_{z, d}=\bar{\omega}_{z, d}
$$

where, $\tau$ is the time constant for the filter.

Two types of actuators that generate yaw moment to the vehicle are considered: a front tire steering angle and a yaw moment by independent tire braking forces:

$$
\begin{gathered}
u_{1}=\delta \\
u_{2}=\frac{T_{W}}{2}\left[\left(F_{x, f_{R}}-F_{x, f_{L}}\right) \cos \delta+\left(F_{x, r_{R}}-F_{x, r_{L}}\right)\right]+a\left(F_{x, f_{L}}+F_{x, f_{R}}\right) \sin \delta
\end{gathered}
$$

Substituting Equation (5.65) into (5.55) and applying the small steering angle assumption results in the following:

$$
\dot{\omega}_{z}=\frac{1}{I_{z z}} u_{2}-\frac{b}{I_{z z}}\left(F_{y, r_{L}}+F_{y, r_{R}}\right)+\frac{a}{I_{z z}}\left(F_{y, f_{L}}+F_{y, f_{R}}\right)-\frac{T_{W}}{2 \cdot I_{z z}}\left(F_{y, f_{R}}-F_{y, f_{L}}\right) \cdot u_{1}
$$

Using Equation (5.61) and rearranging the terms in Equation (5.66), we obtain the desired steering angle and the desired yaw moment:

$$
\begin{gathered}
u_{\text {steer }, F B}=\frac{-2}{T_{W} \cdot\left(\hat{F}_{y, f_{R}}-\hat{F}_{y, f_{L}}\right)} \cdot\left[\begin{array}{l}
b\left(\hat{F}_{y, r_{L}}+\hat{F}_{y, r_{R}}\right)-a\left(\hat{F}_{y, f_{L}}+\hat{F}_{y, f_{R}}\right)-\hat{u}_{2} \\
+I_{z z} \cdot\left\{\dot{\omega}_{z, d}-k_{2}\left(\omega_{2}-\omega_{z, d}\right)\right\}
\end{array}\right] \\
u_{\text {brake }, F B}=-a \cdot\left(\hat{F}_{y, f_{L}}+\hat{F}_{y, f_{R}}\right)+b \cdot\left(\hat{F}_{y, r_{L}}+\hat{F}_{y, r_{R}}\right)+\frac{T_{W}}{2} \cdot\left(\hat{F}_{y, f_{R}}-\hat{F}_{y, f_{L}}\right) \cdot \hat{u}_{1} \\
+I_{z z} \cdot\left\{\dot{\omega}_{z, d}-k_{2}\left(\omega_{2}-\omega_{z, d}\right)\right\}
\end{gathered}
$$

The desired yaw moment is converted into variations of brake forces at each wheel. Application of the left or right side brakes is determined by the direction of the desired yaw moment. Then the yaw moment from Equation (5.68) can be considered into two cases: 
When $u_{\text {brake, } F B}>0, \quad u_{\text {brake }, F B}=\left(-\frac{T_{W}}{2} \cos \delta+a \sin \delta\right) \cdot F_{x, f_{L}}-\frac{T_{W}}{2} \cdot F_{x, r_{L}}$

When $u_{\text {brake, } F B}<0, \quad u_{\text {brake }, F B}=\left(\frac{T_{W}}{2} \cos \delta+a \sin \delta\right) \cdot F_{x, f_{R}}+\frac{T_{W}}{2} \cdot F_{x, r_{R}}$

From Equations (5.69) and (5.70), the braking control pressure amounts are approximated by the linear relationship between wheel brake pressure and corresponding brake force. Then the desired yaw moment has a relationship with the braking pressure $(P)$ as

$$
u_{\text {brake }, F B}=\kappa_{f} \cdot P_{f_{L \text { or } R}}+\kappa_{r} \cdot P_{r_{L \text { or } R}}
$$

where, $\kappa_{f}$ and $\kappa_{r}$ are constant gains.

To determine the front and rear wheel brake pressure, a brake proportioning rule is followed:

$$
P_{r_{L}}=\rho \cdot P_{f_{L}}, P_{r_{R}}=\rho \cdot P_{f_{R}} \quad \text { where, } \rho \in(0,1)
$$

Then, the equation becomes

$$
\text { When } u_{\text {brake, } F B}>0, \quad P_{f_{L}}=u_{\text {brake }, F B} /\left(\kappa_{f}+\kappa_{r} \cdot \rho\right), \quad P_{r_{L}}=\rho \cdot P_{f_{L}}
$$

When $u_{\text {brake, } F B}<0, \quad P_{f_{R}}=u_{\text {brake }, F B} /\left(\kappa_{f}+\kappa_{r} \cdot \rho\right), \quad P_{r_{R}}=\rho \cdot P_{f_{R}}$ 


\subsubsection{Simulation Result of the Preemptive Control Algorithm}

The simulation results shown in Figure 5.31 and Figure 5.32 demonstrate the feasibility of this preemptive steering control action. It is assumed that the striking vehicle has a heading angle of $25^{\circ}$ when the collision occurs with a speed of $33.5 \mathrm{~m} / \mathrm{s}$ (120 kph or $75 \mathrm{mph})$. The struck vehicle was running straight at $30 \mathrm{~m} / \mathrm{s}(108 \mathrm{kph}$ or 67 $\mathrm{mph}$ ). The counter-yaw rate is generated 0.5 seconds before the collision happens. On the other hand, the vehicle without control shows a large lateral deviation from the original course and a substantial slip angle develops as shown in Figure 5.31. It is also noted that the steering and braking control after the impact ('PISC-without-preemptive') shows larger lateral deviation than the proposed preemptive control. Figure 5.32 shows the time history comparison. The collision impact generates $-1.1 \mathrm{~g}$ lateral peak acceleration and $51 \%$ peak yaw rate without control and causes a large lateral deviation and sideslip angle.

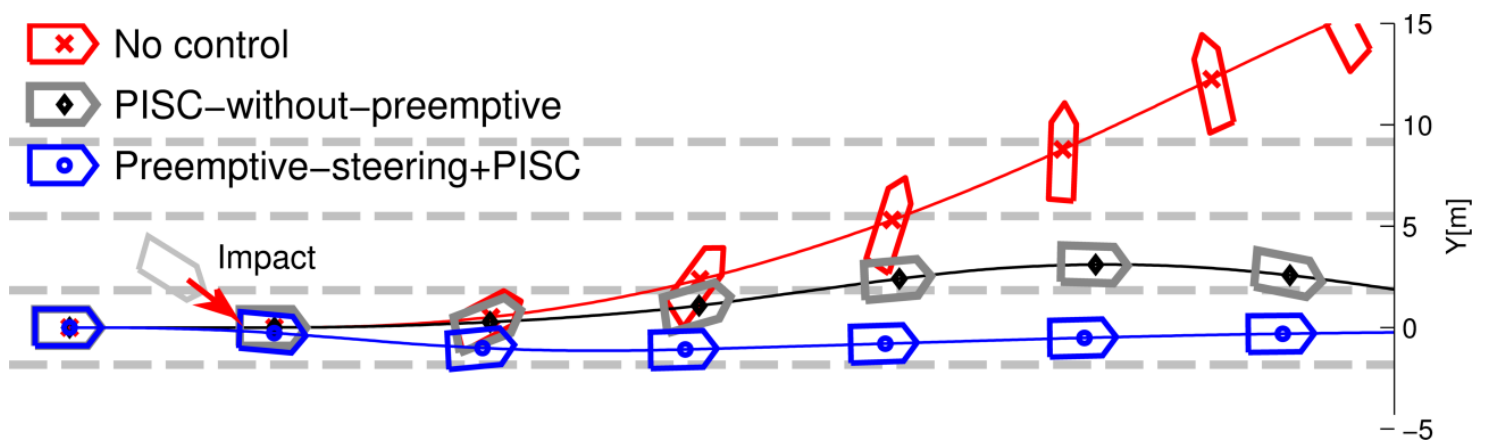

Figure 5.31 Trajectories of the vehicles with and without the proposed preemptive steering control

The preemptive steering control is triggered before the impact at $t=1.95$ seconds, which results in $-0.39 \mathrm{~g}$ lateral acceleration and $-12 \%$ yaw rate. With the preemptive control, the yaw rate and the sideslip angle converge to zero with less oscillation than that of PISC. The wheel steering control outputs are shown in Figure 5.32 (b). It is noted that the proposed system reduces both front and rear tire slip angles. Considering that the control authority of steering and braking can be dramatically reduced when the tire is saturated, keeping the tire slip angle small is beneficial. 


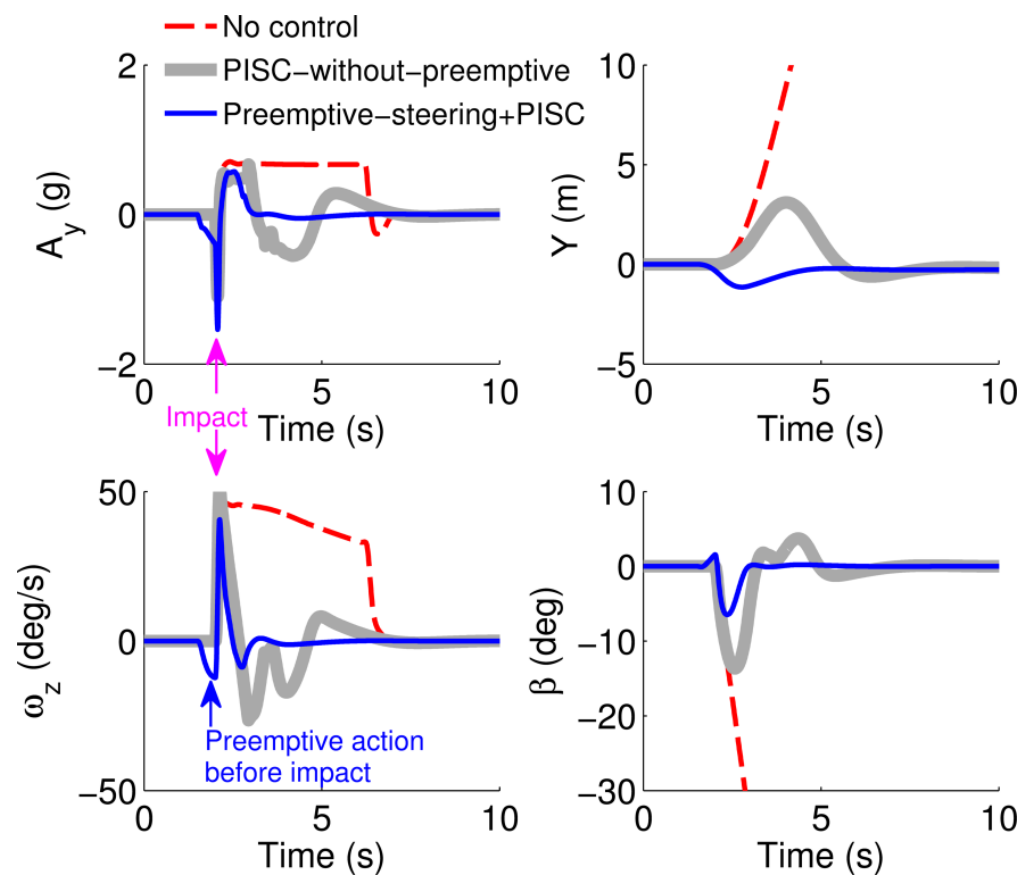

(a)
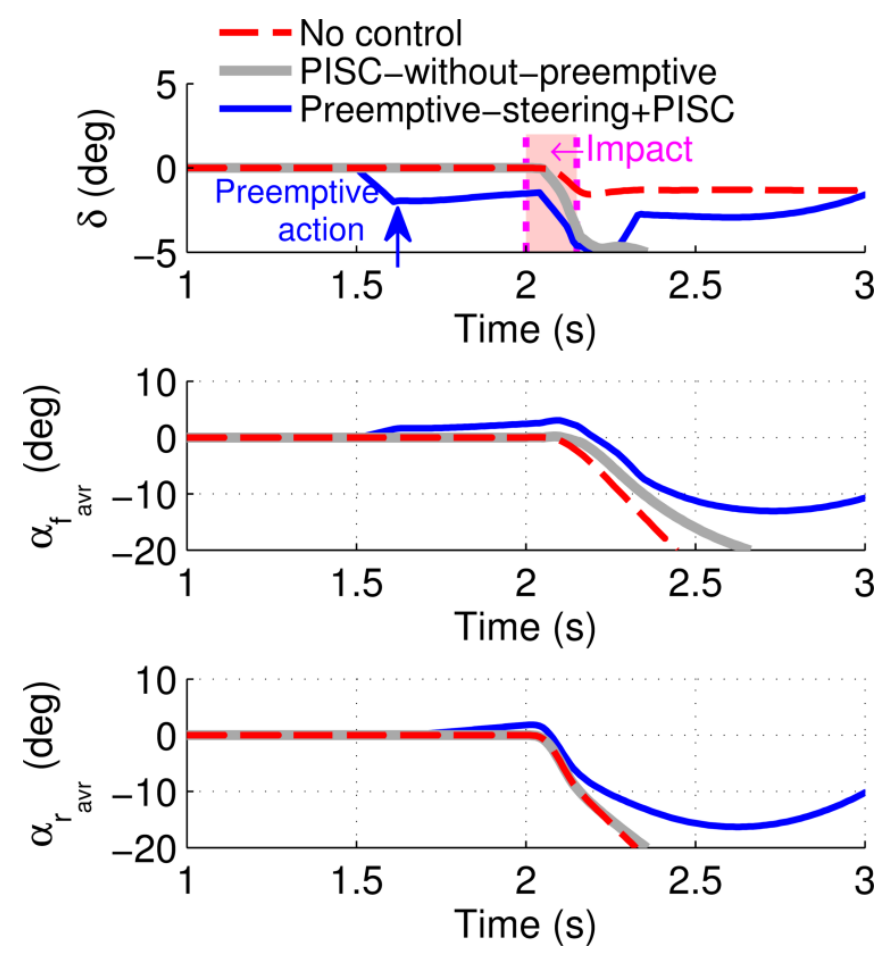

(b)

Figure 5.32 Test result comparison. (vehicle motion without control, with a steering and brake control during and after the impact (PISC), and with preemptive counter-steering control before the impact ) 


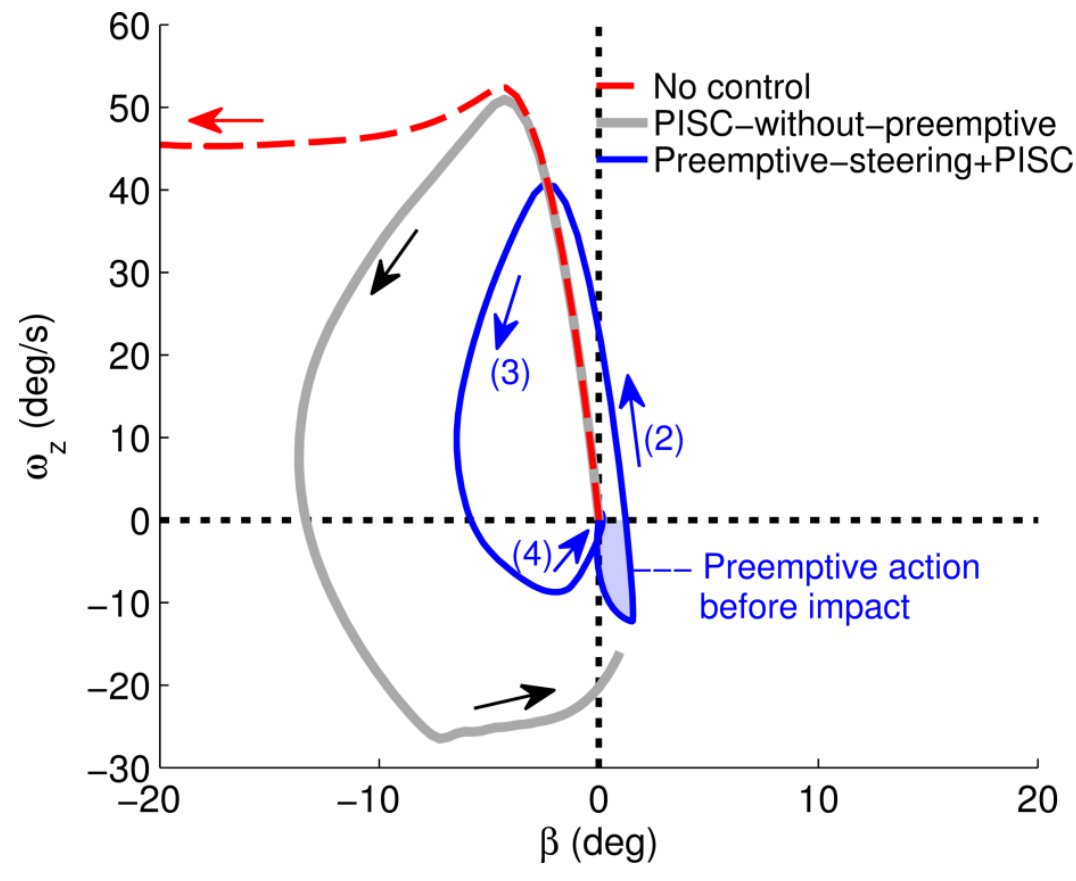

Figure 5.33 Evolution of the vehicle motion trajectories on the slip angle-yaw rate phase plane $\left(\beta-\omega_{z}\right)$ after an impact

In the phase plane plot shown in Figure 5.33, it is obvious that the proposed preemptive control suppresses the yaw and sideslip motion and improves the convergence to the origin. These results show that the subsequent control performances with the preemptive action become much more effective when the preemptive control reduces the tire slip angles and yaw rate after the impact. Therefore, the proposed algorithm can be understood as an enabler to other differential-braking based post-impact control strategy presented in [28, 41]. Figure 5.34 compares two sets of control performances under the same simulation conditions. The plot shows the lateral displacement is reduced up to $90 \%$ by the preemptive steering $\left(Y_{\max , \text { Pre }}\right)$. In other words, the preemptive steering can effectively attenuate the lateral displacement. The reduction in maximum yaw rate is less but still significant. Overall, the risk of secondary collision is expected to be reduced significantly. 


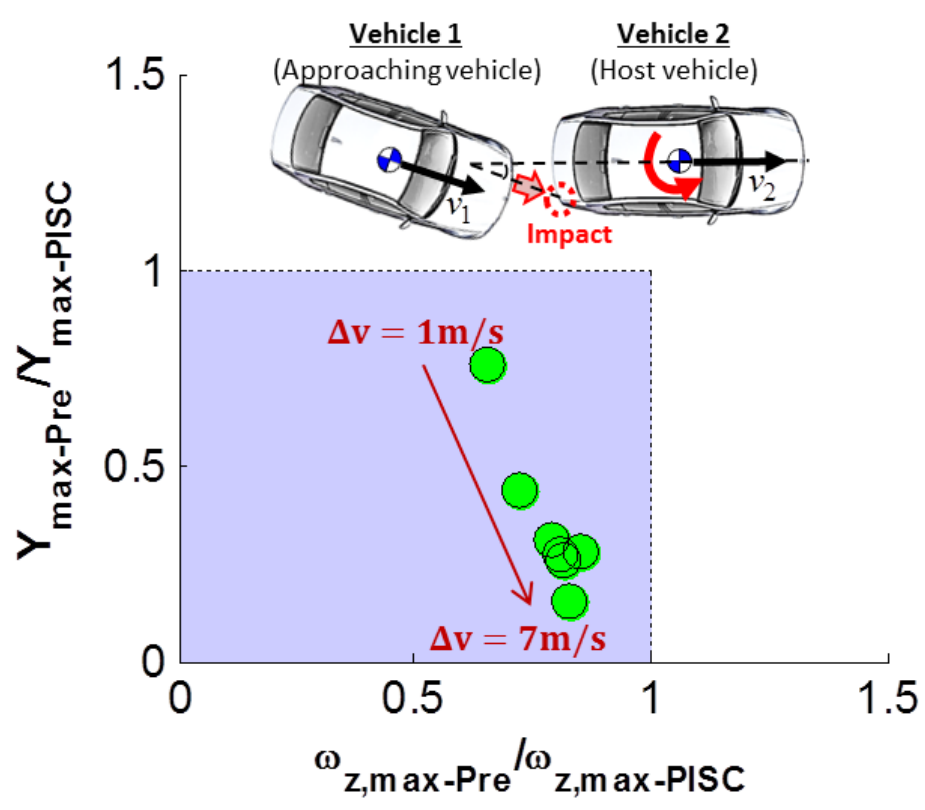

Figure 5.34 Maximum lateral deviation and maximum yaw rate comparison with and without the preemptive steering control:

speed of vehicle $1\left(v_{1}=31 \sim 37 \mathrm{~m} / \mathrm{s}\right)$, speed of vehicle $2\left(v_{2}=30 \mathrm{~m} / \mathrm{s}\right)$, and collision angle between vehicles $\left(\theta=25^{\circ}\right)$

\subsubsection{Preemptive Differential Braking Control}

\subsubsection{Brake Control Strategy}

An alternative way of generating vehicle yaw moment preemptively is considered in this section. ESC, the brake control system that helps to "steer" the vehicle [90] is already available on all new light duty vehicles in the US. Other active safety functions, which utilizes automatic braking, can take advantage of the ESC hardware [91, 92]. The preemptive brake-steering function can be realized utilizing the ESC hardware, instead of adding an active steering control system. By doing so, similar performance is expected.

As analyzed in Figure 5.23, steering can produce more yaw moment than braking. Nonetheless, the braking action is still helpful to generate yaw moment on the vehicle. For example, in Figure 5.35, brakes with the left front and rear tires generate positive yaw moment (counter-clockwise direction) while brakes with the right front and rear tires do negative yaw moment (clockwise direction). Figure 5.36 also illustrates the brake effects from the right tires. So, control actuators can be selected with a simple condition: When 
the desired yaw moment is positive, brakes are applied to left tires. Else, apply brake to the right tires.
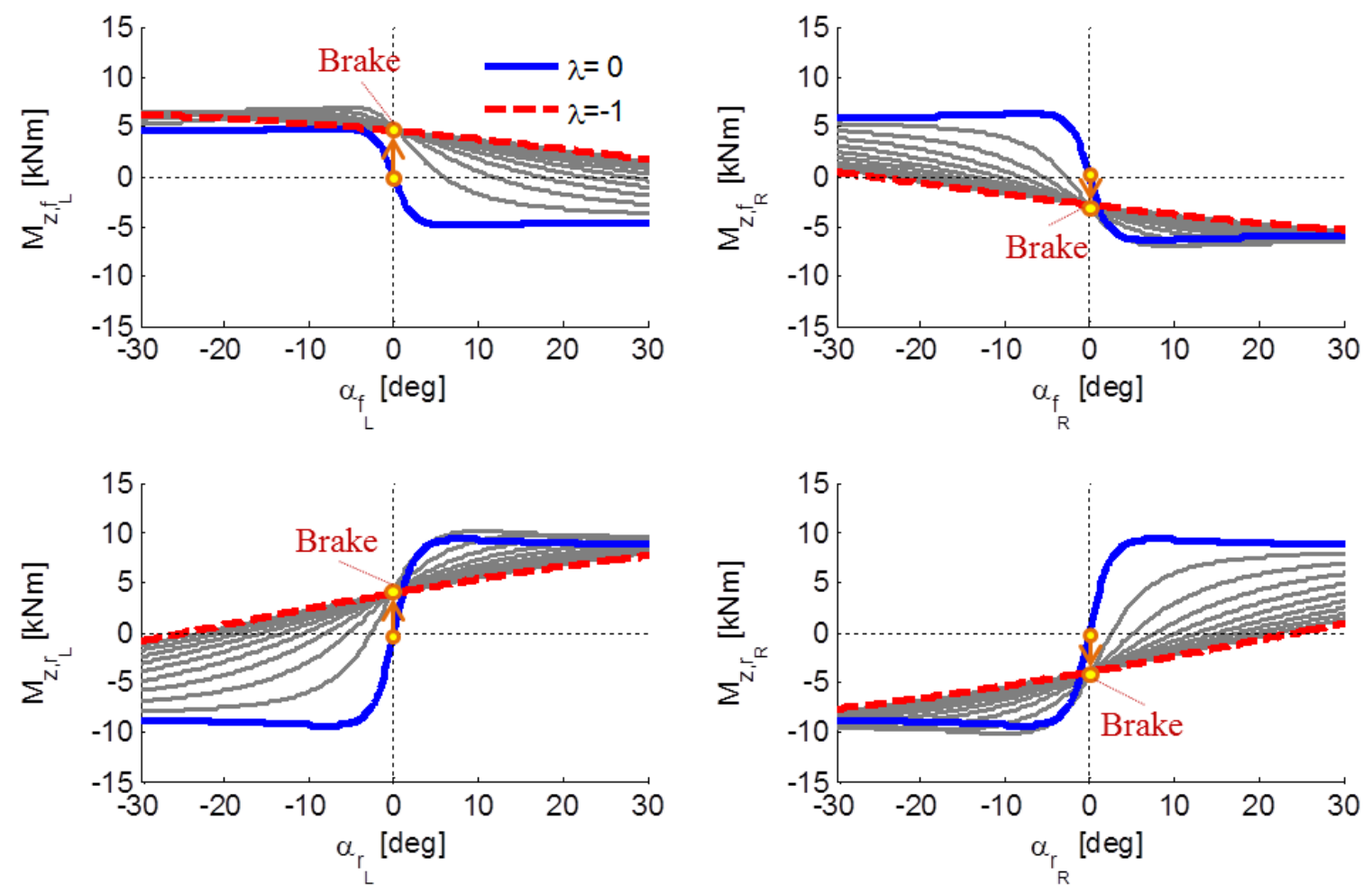

Figure 5.35 Yaw moment that can be generated by each tire (Orange circles and arrows illustrate the change of yaw moment with braking at zero slip angle. Gray lines are yaw moment in $-1<\lambda<0$.)

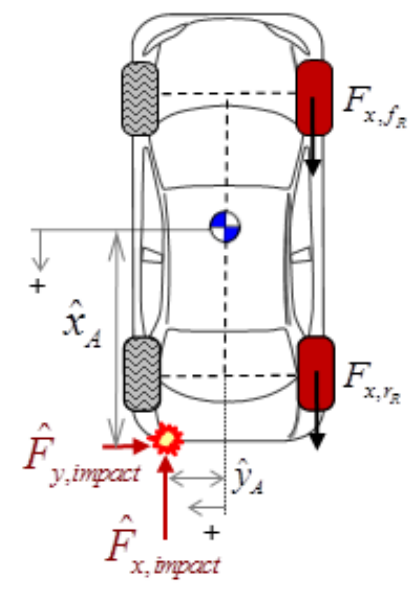

Figure 5.36 Illustration of the preemptive differential brake function applying the front and rear right tire brakes to generate vehicle yaw moment 
To determine the desired yaw moment as a preemptive control, expected collision forces in the Equation (5.52) and the expected contact location $\left(\hat{x}_{A}, \hat{y}_{A}\right)$ are used. Recall, we consider the situation before a collision occurs in order to negate the resulting vehicle motion due to the impact. In addition, we still assume that some sensors detect an approaching vehicle and provide relative speed between vehicles and expected collision location. Using the geometry as shown Figure 5.36, the expected yaw moment from the expected collision force can be calculated as

$$
\hat{M}_{z}=\hat{F}_{y, \text { impact }} \cdot \hat{x}_{A}-\hat{F}_{x, \text { impact }} \cdot \hat{y}_{A}
$$

Then, the desired yaw moment is the same amount of the expected yaw moment, but the opposite direction.

$$
M_{z, \text { desired }}=-\hat{M}_{z}
$$

Similar to the brake control determination in Equations (5.73) and (5.74), the preemptive brake control amounts are calculated from

$$
\begin{array}{lll}
\text { When } M_{\text {desired }}>0, & P_{f_{L}}=M_{z, \text { desired }} /\left(\kappa_{f}+\kappa_{r} \cdot \rho\right), & P_{r_{L}}=\rho \cdot P_{f_{L}} \\
\text { When } M_{\text {desired }}<0, & P_{f_{R}}=M_{z, \text { desired }} /\left(\kappa_{f}+\kappa_{r} \cdot \rho\right), & P_{r_{R}}=\rho \cdot P_{f_{R}}
\end{array}
$$

\subsubsection{Preemptive differential brake simulation results}

The simulation conditions are the same as in Figure 5.31. Figure 5.37 shows the brake control actions at 0.5 seconds before the collision is initiated. It is noted that the proposed control action leads to small tire slip angle and rapidly reduces both front and rear tire slip angles. Figure 5.38 also shows similar trajectories compared to the result in Figure 5.31. The preemptive brake-steer function keeps the tire slip angle small and effectively stabilizes the vehicle. 

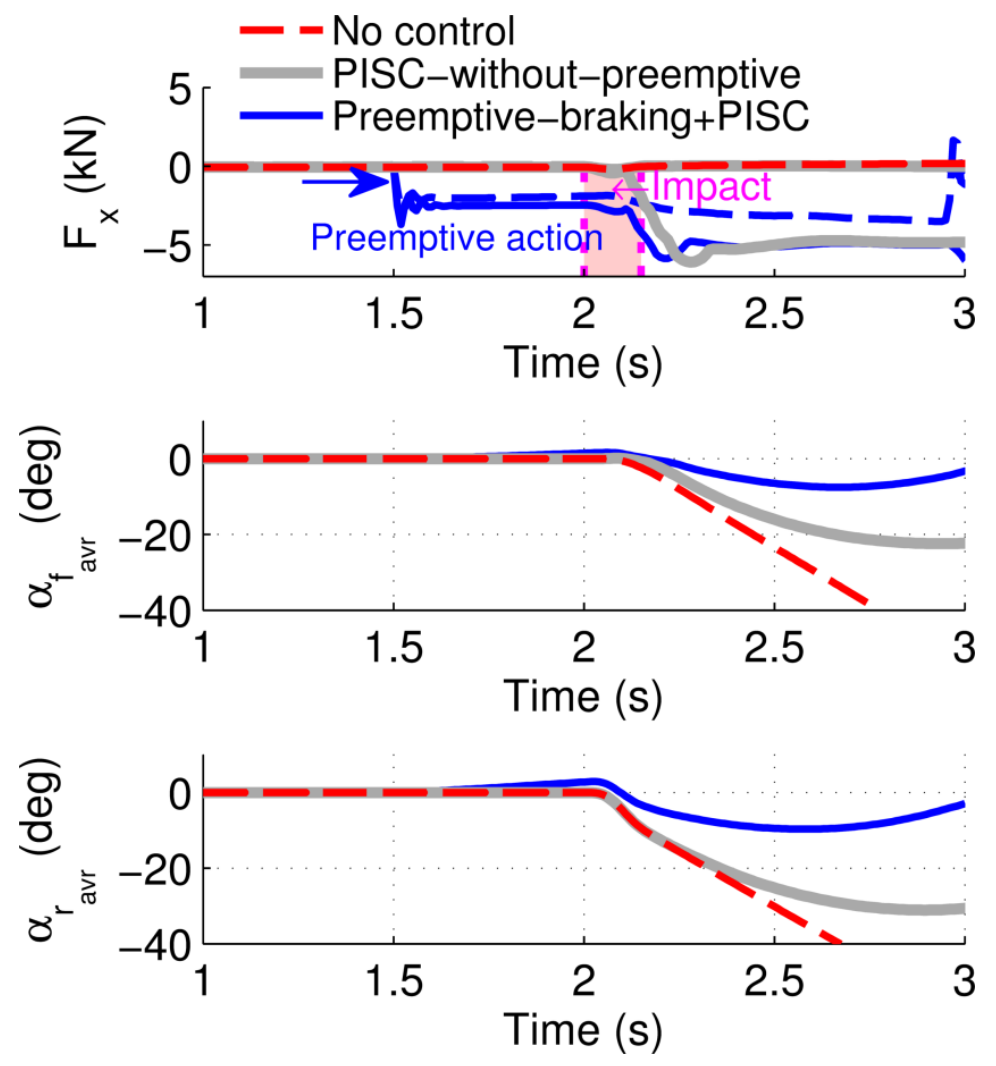

Figure 5.37 Preemptive differential brake test result

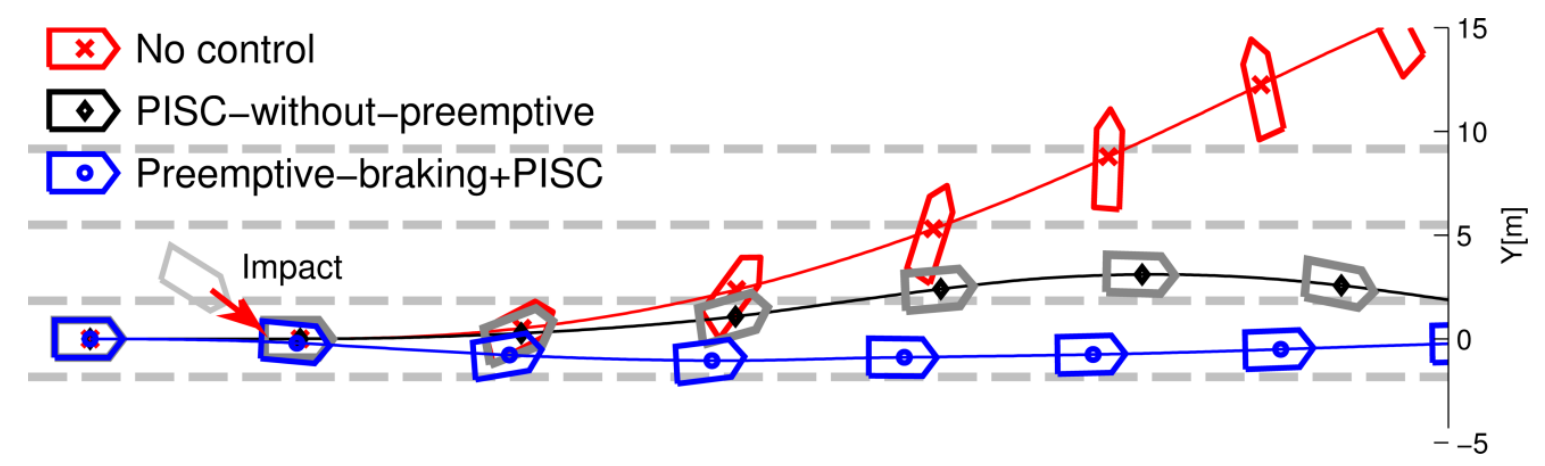

Figure 5.38 Trajectories of the vehicles with the preemptive differential brake function 


\section{CHAPTER 6 \\ CONCLUSION AND FUTURE WORK}

\subsection{Conclusion}

The objective of this study is to develop a vehicle control system to mitigate or avoid secondary collisions. The research work for achieving this objective consists of three major tasks: (1) crash impact and vehicle motion prediction; (2) desired vehicle motion determination; and, (3) a control algorithm to achieve the desired vehicle motion.

To estimate the vehicle response after a collision event, a collision estimation model is first developed. Then, a model-based estimation process is developed which: 1) estimates the crash impulse magnitude and location; and 2) predicts the entire force profile and vehicle responses during and immediately after the collision. Uncertainty of crash time duration is managed by detecting the inflection point of the estimated impulse curve. Subsequently, the expected vehicle motion immediately after the collision is predicted.

In contrast to the previous Post-Impact Stability Control (PISC) design reported in [5], the proposed approach takes the full range of the tire force characteristics into account and selects a final heading angle for the vehicle that achieves small lateral displacement and fast decay of the yaw motion. Since a final heading angle in multiples of $180^{\circ}$ with respect to the lanes is beneficial to avoid broadside impacts by other vehicles, and to avoid large lateral displacement, the final heading angle is a major control decision. The desired final heading angles from various initial conditions are selected by performing off-line optimization.

To find a proper control algorithm for post-impact vehicle motion, an LTV-MPC problem is formulated to solve the vehicle-level control. An optimal allocation algorithm is then developed to map the vehicle level virtual control demand to the braking actuators at the four wheels. These two optimization problems exploit the feasible control bounds 
based on the physical constraints so that control signals are always implementable. To determine the control constraints based on the vehicle states, coupling between the tire longitudinal and lateral forces and actuation limits are considered. An LTV-MPC problem is constructed using direct on-line linearization of the nonlinear system model, and extends the system operating range beyond the linear tire force region. Because the objective function for the LTV-MPC problem is formulated in a quadratic form, computational complexities are reduced. Simulation results show that independent 4wheel braking actions can lead a vehicle to a safe heading angle while reducing the lateral deviation, which can reduce the risk of secondary collisions. Comparisons with other control methods indicate that the proposed control method achieves better positional and directional safety. The main reason for the superior performance is because the controller is designed to consider multiple equilibria on the phase plane instead of trying to bring the vehicle to the origin.

To achieve faster control decision and reduce computational load in real-time implementation, a rule-based control strategy is proposed. The rule-based controller is constructed to mimic the behaviors of the LTV-MPC control.

Lastly, a more effective control concept is proposed by taking preemptive control action before a collision happens. A preemptive steering control is designed to counteract the imminent impact. By doing so, the proposed control concept is able to reduce yaw rate and side slip much faster than all reactive control strategies. In addition, a preemptive brake-steer concept is also proposed as a more realistic implementation by utilizing ESC hardware that is already available on all light duty vehicles in the US today. The performance of the brake-steer was found to be very effective, and achieves similar performance to the preemptive steering algorithm. 


\subsection{Future Work}

Several key areas need to be accomplished before the proposed algorithms can be realized:

(1) Based on the predicted vehicle motion after a collision impact, the final heading is selected. However, real implementations may have more. For example, the road may not be wide enough or other vehicles may be present which can prevent safe execution of the maneuver leading to a heading angle in multiples of $180^{\circ}$. A possible way to address this issue is to incorporate more complex geometric conditions to the optimization problem so that road boundaries and other vehicle positions are included as constraints. Then, this problem extends to a path planning method that selects a desired path among a set of feasible trajectories, as described in [93-96].

(2) Tire forces and slip angles are critical for the proposed estimation and control algorithms, but they may be affected by road conditions. Sophisticated road friction estimation approaches, such as the algorithm presented in [97], may be necessary. Moreover, vehicle sideslip angle estimation is not trivial. Robust sideslip angle estimation schemes, such as those presented in [98, 99], may be necessary to ensure the robustness of the system performance.

(3) The collision estimation error can be affected by the model uncertainties and then the desired control states should be reconsidered based on the estimation errors. Thus, sensitivity analysis on the relationship between parameter variations in vehicle model and the resulting vehicle motion predictions needs to be examined. In addition, because the collision estimation model relies on sensor information, the effects of the sensor latency need to be studied more.

(4) Because of the constraint conditions, guaranteeing the stability of MPC controller is a challenging issue. As studied for the stability of LTV-MPC in [100], exploring input bounds for control stability could provide a guideline to design a controller. Moreover, study of criteria for choosing MPC previewing time horizon and tuning matrices could be helpful to present better control performances. 


\section{BIBLIOGRAPHY}

[1] NHTSA, "Traffic Safety Facts," US Department of Transportation 2012.

[2] J. Bahouth and K. Digges, "Characteristics of Multiple Impact Crashes That Produce Serious Injuries," in Proceedings of the 19th International Technical Conference on the Enhanced Safety of Vehicles, Washington DC, USA, 2005.

[3] U. Sander, K. Mroz, O. Boström, and R. Fredriksson, "The effect of prepretensioning in multiple impact crashes," in 21st International Technical Conference on the Enhanced Safety of Vehicles (ESV), 2009.

[4] A. Togawa, D. Murakami, H. Saeki, C. Pal, and T. Okabe, "An Insight into Multiple Impact Crash Statistics to Search for Future Directions of CounterApproaches," in 22th International Technical Conference on the Enhanced Safety of Vehicles (ESV), 2011.

[5] J. Zhou, "Active Safety Measures for Vehicles Involved in Light Vehicle-toVehicle Impacts," Ph.D., Mechanical Engineering, The University of Michigan, 2009.

[6] A. Eigen and W. Najm, "Problem definition for pre-crash sensing advanced restraints," DOT HS, vol. 811, p. 114, 2009.

[7] T. Ayres, L. Li, D. Trachtman, and D. Young, "Passenger-side rear-view mirrors: driver behavior and safety," International journal of industrial ergonomics, vol. 35, pp. 157-162, 2005.

[8] R. Bogenrieder, M. Fehring, and R. Bachmann, "PRE-SAFE in rear-end collision situations," in The 21st International Technical Conference on the Enhanced Safety of Vehicles Conference (ESV), Stuttgart, Germany, 2009.

[9] D. Yang, B. Jacobson, and M. Lidberg, "Benefit prediction of passenger car post impact stability control based on accident statistics and vehicle dynamics simulations," in Proceedings of 21st IAVSD Symposium on Dynamics of Vehicles on Roads and Tracks, 2009.

[10] National Automotive Sampling System (NASS)-Crashworthiness Data System $(C D S)$. Available: http://www-nass.nhtsa.dot.gov/nass/cds/SearchForm.aspx

[11] J. K. Hedrick, Y. Chen, and S. Mahal, "Optimized vehicle control/communication interaction in an automated highway system," 2001. 
[12] Autonomous vehicles: safety and performance requirements, California Senate Bill No. 1298, 2012.

[13] J. Lenard and R. Frampton, "Two-Impact Crashes-Implications for Occupant Protection Technologies," in Proceedings of 18th International Technical Conference on the Enhanced Safety of Vehicles, Nagoya, Japan, 2003.

[14] B. Fildes, J. C. Lane, J. Lenard, and A. Vulcan, "Passenger cars and occupant injury: side impact crashes," 1994.

[15] S. Summers, A. Prasad, and W. T. Hollowell, "NHTSA's research program for vehicle aggressivity and fleet compatibility," in Proceedings of the Seventeenth Annual International Conference on Enhanced Safety of Vehicles, Paper, 2001.

[16] H. C. Gabler, K. Digges, B. N. Fildes, and L. Sparke, "Side impact injury risk for belted far side passenger vehicle occupants," SAE Technical Paper2005.

[17] C. J. Kahane, "An Evaluation of Side Impact Protection," DOT HS 8107482007.

[18] P. Fay, R. Sferco, and R. Frampton, "Multiple impact crashes-consequences for occupant protection measures," 2001.

[19] K. H. Digges and A. M. Eigen, "Crash attributes that influence the severity of rollover crashes," PROGRESS IN TECHNOLOGY, vol. 101, pp. 299-308, 2004.

[20] E. Liebemann, K. Meder, J. Schuh, and G. Nenninger, "Safety and performance enhancement: the Bosch electronic stability control (ESP)," SAE Paper, vol. 20004, pp. 21-0060, 2004.

[21] B.-C. Chen and H. Peng, "Differential-braking-based rollover prevention for sport utility vehicles with human-in-the-loop evaluations," Vehicle System Dynamics, vol. 36, pp. 359-389, 2001.

[22] A. Lie, C. Tingvall, M. Krafft, and A. Kullgren, "The effectiveness of electronic stability control (ESC) in reducing real life crashes and injuries," Traffic injury prevention, vol. 7, pp. 38-43, 2006.

[23] M. Aga and A. Okada, "Analysis of vehicle stability control (VSC)'s effectiveness from accident data," in Proceedings of the 18 th ESV Conference, paper, 2003.

[24] C. M. Farmer, "Effect of electronic stability control on automobile crash risk," Traffic Injury Prevention, vol. 5, pp. 317-325, 2004.

[25] S. A. Ferguson, "The effectiveness of electronic stability control in reducing realworld crashes: a literature review," Traffic injury prevention, vol. 8, pp. 329-338, 2007. 
[26] J. N. Dang, "Preliminary results analyzing the effectiveness of Electronic Stability Control (ESC) systems," US Department of Transportation, National Highway Traffic Safety Administration2004.

[27] J. N. Dang, "Statistical Analysis of the Effectiveness of Electronic Stability Control (ESC) Systems-Final Report," 2007.

[28] J. Zhou, J. Lu, and H. Peng, "Vehicle stabilization in response to exogenous impulsive disturbances to the vehicle body," in American Control Conference, 2009. ACC'09., 2009, pp. 701-706.

[29] H. E. Tseng, B. Ashrafi, D. Madau, T. Allen Brown, and D. Recker, "The development of vehicle stability control at Ford," Mechatronics, IEEE/ASME Transactions on, vol. 4, pp. 223-234, 1999.

[30] A. G. Ulsoy, H. Peng, and M. Çakmakci, Automotive control systems: Cambridge University Press, 2012.

[31] A. T. Van Zanten, "Bosch ESP systems: 5 years of experience," SAE Technical Paper2000.

[32] M. Thor, "The Efficiency of Electronic Stability Control after Light Collisions," Master's Thesis, Chalmers University of Technology, Goeteborg, Sweden, 2007.

[33] P. G. Michael, F. C. Leeming, and W. O. Dwyer, "Headway on urban streets: observational data and an intervention to decrease tailgating," Transportation research part F: traffic psychology and behaviour, vol. 3, pp. 55-64, 2000.

[34] N. Kaempchen, B. Schiele, and K. Dietmayer, "Situation assessment of an autonomous emergency brake for arbitrary vehicle-to-vehicle collision scenarios," Intelligent Transportation Systems, IEEE Transactions on, vol. 10, pp. 678-687, 2009.

[35] J. Jansson, "Collision Avoidance Theory: With application to automotive collision mitigation," 2005.

[36] Bosch. Secondary Collision Mitigation: protection against subsequent crashes. Available: http://www.bosch-mobility-solutions.com/

[37] D.-I. A. Häussler, D.-I. R. Schäffler, D.-I. A. Georgi, and I. S. Stabrey, "Networking of airbag and ESP for prevention of further collisions," ATZ worldwide, vol. 114, pp. 22-26, 2012.

[38] Continental. (2012). Continental's Technology Reduces the Probability and Severity of Secondary Collisions. Available: http://www.continentalcorporation.com/www/pressportal_com_en/themes/press_releases/3_automotive_gr oup/chassis_safety/press_releases/pr_2012_07_12_post_crash_braking_en.html 
[39] EURO-NCAP. Audi, Secondary Collision Brake Assist. Available: http://www.euroncap.com/rewards/audi_secondary_collision_brake_assist.aspx

[40] D. Yang, T. Gordon, B. Jacobson, M. Jonasson, and M. Lidberg, "Optimized brakebased control of path lateral deviation for mitigation of secondary collisions," Proceedings of the Institution of Mechanical Engineers, Part D: Journal of Automobile Engineering, vol. 225, pp. 1587-1604, 2011.

[41] D. Yang, T. Gordon, M. Lidberg, M. Jonasson, and B. Jacobson, "Post-impact vehicle path control by optimization of individual wheel braking sequences," in Proceedings of the" 10th International Symposium on Advanced Vehicle Control"(AVEC 10), August 22-26, 2010 in Loughborough, UK., 2010.

[42] B. Kim and H. Peng, "Vehicle Stability Control of Heading Angle and Lateral Deviation to Mitigate Secondary Collisions," in The 11th International Symposium on Advanced Vehicle Control, Seoul, 2012.

[43] Youtube. Audi Advanced Reward - Audi Secondary Collision Brake Assist. Available: https://www.youtube.com/watch?v=VRJ04cUKuDs

[44] Youtube. Euro NCAP's Latest Results: Adavnced Skoda Mult-Collision Brake. Available: $\quad$ http://euroncap.synapticdigital.com/Latest\%20Release/euro-ncapslatest-results/s/fe3d02d0-e7ad-4cad-90b9-9617d39f8f2f

[45] Daimler. (2013). Intelligent Drive: networked with all senses. Available: http://techcenter.mercedes-benz.com/en/pre_safe_plus/detail.html

[46] P. Falcone, F. Borrelli, J. Asgari, H. E. Tseng, and D. Hrovat, "A real-time model predictive control approach for autonomous active steering," Nonlinear Model Predictive Control for Fast Systems, Grenoble, France, 2006.

[47] T. Besselmann and M. Morari, "Autonomous Vehicle Steering Using Explicit LPVMPC," in European Control Conference,(Budapest, Hungary), 2009, pp. 26282633.

[48] K. Kozłowski and D. Pazderski, "Modeling and control of a 4-wheel skid-steering mobile robot," Int. J. Appl. Math. Comput. Sci, vol. 14, pp. 477-496, 2004.

[49] A. Mandow, J. L. Martinez, J. Morales, J.-L. Blanco, A. Garcia-Cerezo, and J. Gonzalez, "Experimental kinematics for wheeled skid-steer mobile robots," in Intelligent Robots and Systems, 2007. IROS 2007. IEEE/RSJ International Conference on, 2007, pp. 1222-1227.

[50] J. Zhou, H. Peng, and J. Lu, "Collision model for vehicle motion prediction after light impacts," Vehicle System Dynamics, vol. 46, pp. 3-15, 2008.

[51] D. Karnopp, Vehicle stability: CRC Press, 2004. 
[52] H. Pacejka, Tyre and vehicle dynamics: Elsevier, 2005.

[53] S. Chang, "A Flexible Hierarchical Model-Based Control Methodology for Vehicle Active Safety Systems," PhD., Mechanical Engineering, The University of Michigan, 2007.

[54] K. L. Talvala and J. C. Gerdes, "Lanekeeping at the limits of handling: Stability via Lyapunov functions and a comparison with stability control," in ASME Dynamic Systems and Control Conference, 2008, pp. 361-368.

[55] R. E. Benton Jr and D. Smith, "A static-output-feedback design procedure for robust emergency lateral control of a highway vehicle," Control Systems Technology, IEEE Transactions on, vol. 13, pp. 618-623, 2005.

[56] T. Kinjawadekar, N. Dixit, G. J. Heydinger, D. A. Guenther, and M. K. Salaani, "Vehicle dynamics modeling and validation of the 2003 Ford Expedition with ESC using CarSim," SAE Technical Paper2009.

[57] Mechanical Simulation Corporation, CarSim Applications. Available: https://www.carsim.com/applications/industry.php

[58] M. Huang, Vehicle crash mechanics: CRC press, 2010.

[59] M. S. Varat and S. E. Husher, "Vehicle impact response analysis through the use of accelerometer data," SAE Tech. Paper 2000-01-0850, 2000.

[60] R. M. Brach, "Modeling of low-speed, front-to-rear vehicle impacts," SAE Tech. Paper 2003-01-0491, 2003.

[61] A. L. Cipriani, F. P. Bayan, M. L. Woodhouse, A. D. Cornetto, A. P. Dalton, C. B. Tanner, et al., "Low-speed Collinear Impact Severity: A Comparison between FullScale Testing and Analytical Prediction Tools with Restitution Analysis," SAE Tech. Paper 2002-01-0540, 2002.

[62] NHTSA (National Highway Traffic Safety Administration), Vehicle Crash Test Database. Available: http://www-nrd.nhtsa.dot.gov/database/veh/veh.htm

[63] M. Klomp, "Longitudinal force distribution and road vehicle handling," Department of Applied Mechanics, Chalmers University of Technology, Gothenburg, Sweden, 2010.

[64] E. Ono, S. Hosoe, and H. D. Tuan, "Bifurcation in vehicle dynamics and robust front wheel steering control," Control Systems Technology, IEEE Transactions on, vol. 6, pp. 412-420, 1998.

[65] S. Inagaki, I. Kshiro, and M. Yamamoto, "Analysis on vehicle stability in critical cornering using phase-plane method," in International Symposium on Advanced 
Vehicle Control (1994: Tsukuba-shi, Japan). Proceedings of the International Symposium on Advanced Vehicle Control 1994, 1994.

[66] R. C. Hoffman, J. L. Stein, L. S. Louca, and K. Huh, "Using the milliken moment method and dynamic simulation to evaluate vehicle stability and controllability," in ASME 2004 International Mechanical Engineering Congress and Exposition, 2004, pp. 173-180.

[67] C. E. Beal, C. G. Bobier, and J. C. Gerdes, "Controlling vehicle instability through stable handling envelopes," in ASME 2011 Dynamic Systems and Control Conference and Bath/ASME Symposium on Fluid Power and Motion Control, 2011, pp. 861-868.

[68] C. G. Bobier and J. C. Gerdes, "Staying within the nullcline boundary for vehicle envelope control using a sliding surface," Vehicle System Dynamics, vol. 51, pp. 199-217, 2013.

[69] A. Hac, D. Nichols, and D. Sygnarowicz, "Estimation of vehicle roll angle and side slip for crash sensing," in SAE International Congress, 2010.

[70] Youtube. A severe secondary crash scene captured on the dashboard camera. Available: https://www.youtube.com/watch?v=ggJ6mbrvbts

[71] Youtube. Police Chase Multiple PIT(Precision Immobilization Technique) Maneuver. Available: https://www.youtube.com/watch?v=1QbBHI031H8

[72] B. Kim and H. Peng, "Vehicle stability control of heading angle and lateral deviation to mitigate secondary collisions," in 11th International Symposium on Advanced Vehicle Control (AVEC), Seoul, Korea, 2012, pp. 48109-2133.

[73] P. S. Agachi, Z. K. Nagy, M. V. Cristea, and A. Imre-Lucaci, Model Based Control: Case Studies in Process Engineering. Weinheim, Germany: Wiley-VCH, 2006.

[74] S. J. Qin and T. A. Badgwell, "A survey of industrial model predictive control technology," Control engineering practice, vol. 11, pp. 733-764, 2003.

[75] D. Hrovat, S. Di Cairano, H. E. Tseng, and I. V. Kolmanovsky, "The development of Model Predictive Control in automotive industry: A survey," in Control Applications (CCA), 2012 IEEE International Conference on, 2012, pp. 295-302.

[76] J. M. Maciejowski, Predictive control with constraints: Pearson education, 2002.

[77] L. Magni, D. M. Raimondo, and F. Allgöwer, Nonlinear model predictive control: towards new challenging applications vol. 384: Springer, 2009.

[78] R. Sharp and H. Peng, "Vehicle dynamics applications of optimal control theory," Vehicle System Dynamics, vol. 49, pp. 1073-1111, 2011. 
[79] P. Falcone, F. Borrelli, J. Asgari, H. E. Tseng, and D. Hrovat, "Predictive active steering control for autonomous vehicle systems," Control Systems Technology, IEEE Transactions on, vol. 15, pp. 566-580, 2007.

[80] B. Kim and H. Peng, "Optimal Vehicle Motion Control to Mitigate Secondary Crashes after an Initial Impact," in ASME Dynamic Systems and Control Conference, San Antonio, TX, 2014.

[81] J. Y. Wong, Theory of ground vehicles: Wiley. com, 2001.

[82] J. Zhou, J. Lu, and H. Peng, "Vehicle Dynamics in Response to the Maneuver of Precision Immobilization Technique," in ASME Dynamic Systems and Control Conference, 2008.

[83] J. A. Bernard, "Use of a rule-based system for process control," Control Systems Magazine, IEEE, vol. 8, pp. 3-13, 1988.

[84] C.-C. Lin, H. Peng, J. W. Grizzle, and J.-M. Kang, "Power management strategy for a parallel hybrid electric truck," Control Systems Technology, IEEE Transactions on, vol. 11, pp. 839-849, 2003.

[85] B. Wu, C.-C. Lin, Z. Filipi, H. Peng, and D. Assanis, "Optimization of power management strategies for a hydraulic hybrid medium truck," in Proceedings of the 2002 Advanced vehicle control conference, 2002, pp. 1-6.

[86] J. Ackermann, D. Odenthal, and T. Bünte, "Advantages of active steering for vehicle dynamics control," in Proceedings of 32nd ISATA, Automotive Mechatronics Design and Engineering, 1999, pp. 263-270.

[87] B. Kim and H. Peng, "Collision Strength Estimation and Preemptive Steering Control for Post-Impact Vehicle Motion Control," in 12th International Symposium on Advanced Vehicle Control, Tokyo, Japan, 2014.

[88] R. Rajamani, Vehicle dynamics and control: Springer, 2011.

[89] J. Hedrick and P. Yip, "Multiple sliding surface control: theory and application," Journal of dynamic systems, measurement, and control, vol. 122, pp. 586-593, 2000.

[90] T. Pilutti, G. Ulsoy, and D. Hrovat, "Vehicle steering intervention through differential braking," Journal of dynamic systems, measurement, and control, vol. 120, pp. 314-321, 1998.

[91] D. Katzourakis, M. Alirezaei, J. C. de Winter, M. Corno, R. Happee, A. Ghaffari, et al., "Shared control for road departure prevention," in Systems, Man, and Cybernetics (SMC), 2011 IEEE International Conference on, 2011, pp. 1037-1043.

[92] M. Ozaki, O. Sadano, and Y. Uemura, "Lane departure prevention apparatus," ed: Google Patents, 2008. 
[93] K. Kant and S. W. Zucker, "Toward efficient trajectory planning: The path-velocity decomposition," The International Journal of Robotics Research, vol. 5, pp. 72-89, 1986.

[94] R. Bis, H. Peng, and A. G. Ulsoy, "Velocity occupancy space: autonomous navigation in an uncertain, dynamic environment," International Journal of Vehicle Autonomous Systems, vol. 10, pp. 41-66, 2012.

[95] Y. Yoon, J. Shin, H. J. Kim, Y. Park, and S. Sastry, "Model-predictive active steering and obstacle avoidance for autonomous ground vehicles," Control Engineering Practice, vol. 17, pp. 741-750, 2009.

[96] S. J. Anderson, S. B. Karumanchi, and K. Iagnemma, "Constraint-based planning and control for safe, semi-autonomous operation of vehicles," in Intelligent Vehicles Symposium (IV), 2012 IEEE, 2012, pp. 383-388.

[97] C. Ahn, H. Peng, and H. E. Tseng, "Robust estimation of road friction coefficient using lateral and longitudinal vehicle dynamics," Vehicle System Dynamics, vol. 50, pp. 961-985, 2012.

[98] J.-H. Yoon and H. Peng, "Robust Vehicle Sideslip Angle Estimation Through a Disturbance Rejection Filter That Integrates a Magnetometer With GPS," IEEE Transactions on Intelligent Transportation Systems, vol. 15, 2014.

[99] J. Ryu and J. C. Gerdes, "Integrating inertial sensors with global positioning system (GPS) for vehicle dynamics control," Journal of Dynamic Systems, Measurement, and Control, vol. 126, pp. 243-254, 2004.

[100] P. Falcone, F. Borrelli, H. E. Tseng, J. Asgari, and D. Hrovat, "Linear time-varying model predictive control and its application to active steering systems: Stability analysis and experimental validation," International journal of robust and nonlinear control, vol. 18, pp. 862-875, 2008. 